University of Louisville

ThinkIR: The University of Louisville's Institutional Repository

\title{
Electrochemically-modulated surface plasmon waves at a nano- scale for investigations and applications of redox assemblies.
}

Aymen Hasan Qatamin

University of Louisville

Follow this and additional works at: https://ir.library.louisville.edu/etd

Part of the Other Physics Commons

\section{Recommended Citation}

Qatamin, Aymen Hasan, "Electrochemically-modulated surface plasmon waves at a nano-scale for investigations and applications of redox assemblies." (2020). Electronic Theses and Dissertations. Paper 3575.

https://doi.org/10.18297/etd/3575

This Doctoral Dissertation is brought to you for free and open access by ThinkIR: The University of Louisville's Institutional Repository. It has been accepted for inclusion in Electronic Theses and Dissertations by an authorized administrator of ThinkIR: The University of Louisville's Institutional Repository. This title appears here courtesy of the author, who has retained all other copyrights. For more information, please contact thinkir@louisville.edu. 


\title{
ELECTROCHEMICALLY-MODULATED SURFACE PLASMON WAVES AT THE NANO-SCALE FOR INVESTIGATIONS AND APPLICATIONS OF REDOX ASSEMBLIES
}

\author{
By \\ Aymen Hasan Qatamin \\ B.S., Al-Mustansiriya University, 2001 \\ M.S., Lund University, 2012 \\ M.S., University of Louisville, 2018

\begin{abstract}
A Dissertation
Submitted to the Faculty of the

College of Arts and Science at the University of Louisville

in Partial Fulfillment of the Requirements

for the Degree of
\end{abstract}

Doctor of Philosophy in Physics

Department of Physics and Astronomy

University of Louisville

Louisville, Kentucky

August 2020 
Copyright 2020 by Aymen Hasan Qatamin

All rights reserved 



\section{ELECTROCHEMICALLY-MODULATED SURFACE PLASMON WAVES AT THE NANO-SCALE FOR INVESTIGATIONS AND APPLICATIONS OF REDOX ASSEMBLIES}

By

Aymen Hasan Qatamin

B.S., Al-Mustansiriya University, 2001

M.S., Lund University, 2012

M.S., University of Louisville, 2018

A Dissertation Approved on

July 14,2020

By the Following Dissertation Committee:

\begin{tabular}{c}
\hline Dr. Sergio B. Mendes \\
\hline Dr. Gamini Sumanasekera \\
\hline Dr. Serban Smadici \\
\hline Dr. Martin G. O'Toole
\end{tabular}




\section{DEDICATION}

I lovingly dedicate this dissertation to my beloved parents, who have been my constant source of strength inspiration and who have sacrificed in order to see me a distinguished person, thank you for your encouragement, prayers and loving. To Marwa, my wonderful wife, my best friend, my love... who has shared with me each step of the way and without her support this dreaming would not have been made possible, thank you for your motivation, exemplary patience, and support during this journey. To my father and mother in law for their prayers and support us during these years. To my lovely

daughters, Mayar, Shemah, and Salma, you have given me the power and strength to achieve greatness, I love you forever. To my brothers and sisters, I love you all. 


\section{ACKNOWLEDMENTS}

I would like to acknowledge and thanks the help and support of all people who made this dream to be real. I would like to express my deep thanks and gratitude to my supervisor Dr. Sergio B. Mendes for his effort and knowledge, constant support, and guidance in all the time of my research journey, thank you for your help, patience, and unlimited support.

Special thanks to my friend Jafar Ghithan for effort me his knowledge and help all the time during my research work. I also would like to thank my dissertation committee, Dr. Gamini Sumanasekera, Dr. Serban Smadici, and Dr. Martin G. O’Toole.

My special thanks to Dr. Martin G. O'Toole and his group members Dr. Monica Moreno and Betty M. Nunn for their constant support during my research.

I am grateful to my brother Moath and his family for sharing the wonderful years with us during our staying here in Louisville.

Special thanks also goes to Dr. Jayanthi Chakram, Dr. Chris Davis, Joshua Rimmer and Mary Gayle from the Department of Physics and Astronomy for their help. Finally, I would like to thank my fellow colleagues of photonics research labs, Shadi, Mohammad, and Keenan for their consistent support and good wishes. 


\begin{abstract}
ELECTROCHEMICALLY-MODULATED SURFACE PLASMON WAVES AT THE NANO-SCALE FOR INVESTIGATIONS AND APPLICATIONS OF

REDOX ASSEMBLIES
\end{abstract}

Aymen Hasan Qatamin

July 14,2020

In this thesis, an electrochemically-modulated surface plasmon resonance (ECSPR) technique was developed for challenging investigations and applications in different redox assemblies. The principle of the technique is based on the nano-scale confined and sensitive interplay between a surface plasmon wave and a redox-active probe featuring an optical transition when undergoing a faradaic redox process. Such faradaic process is electrochemically controlled by modulating the surface electric potential of the SPR platform to create an optical output signal that is substantially immune to the negative impacts of background environment and enabling the identification and quantification minute properties linked to a molecular event of interest.

First, the structure of the EC-SPR platform and the ideal experimental conditions to optically interrogate it were investigated. Different strategies were examined for the ideal SPR configuration to enhance the spectroelectrochemical response imprinted into the optical signal. A redox active probe, the cytochrome $c$ protein, was used as a model system to report the performance of the EC-SPR device and to confirm its suitability for spectroelectrochemical measurements. Experimental measurements of SPR reflectance curves and numerical analysis of the experimental data based on a developed Mathematica 
code were implemented to support the rationale design of SPR configurations aiming to maximize the optical response linked to a redox process and to ultimately reach a high performance for the EC-SPR platform.

Next, a combination of the EC-SPR device and the optical impedance spectroscopy technique (OIS) was demonstrated to provide an effective analytical path to study electron transfer rates in redox species. When compared to competing technologies, the major advantages of this combination are the ability to implement the device over a broad range applied electric potentials and to reach high modulation frequencies. These features increase the capability of the EC-SPR technology for investigations and applications of fast reaction rate constants for a wider range of surface-adsorbed redox species. The capability of the EC-SPR device for determining electron-transfer rates was experimentally demonstrated here. The electron-transfer rate of a redox probe, cytochrome c protein, immobilized at different functionalized assemblies on the plasmonic interface were investigated. The results show that each functionalized layer has a strong impact on the electron-transfer rate of a redox probe interacting with the functionalized electrode of the EC-SPR platform. The quantification and understanding of the electrochemical behavior of these functionalized layers and the ability of the EC-SPR platform to monitor the electron-transfer rate of biomolecular assemblies provide the groundwork for creating a novel biosensing strategy of high performance, which is described below.

Using the EC-SPR device, a novel biosensing methodology that uses redox reactions, controlled by a surface electrode, to electrically modulate the optical output of a molecular probe featured in a sandwich-style bioassay was developed and demonstrated here. A monoclonal antibody was bound to the EC-SPR platform to create an interface that was 
prepared to recognize and capture a target protein. Once these antigens were captured on the device surface, they promoted the immobilization of a polyclonal secondary antibody that has been labeled with a methylene blue (MB) dye. A hemagglutinin (HA) protein from the H5N1 avian influenza A virus was used to demonstrate the capability of the EC-SPR device for the detection and quantification of a critical influenza antigen. In addition, the capability of the EC-SPR device to directly detect and reproducibly identify the presence of gram-negative bacteria in complex samples (whole blood and plasma) was successfully demonstrated. The experimental results with viral and endotoxin biomaterials have produced outstanding limits of detection in the pico-molar range. Using this approach, only the modulated photon output is surface-coupled, offering a means to significantly reduce the effects of background signal that comes from the substrate or fluid media.

Overall, the EC-SPR technology provides an inexpensive and easy-to-use transduction platform that offers a simpler path towards I) investigating a variety of redoxtransduced events with extremely low surface densities and a small difference in their molar absorptivities, II) high sensitivity detection of multiplexed targets with a small footprint that only requires a small amount of sample material at low concentrations of a target antigen. 


\section{TABLE OF CONTENTS}

CONTENTS

PAGE

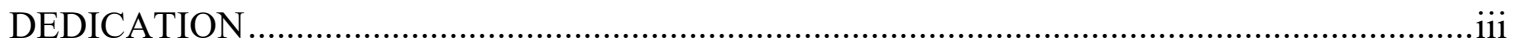

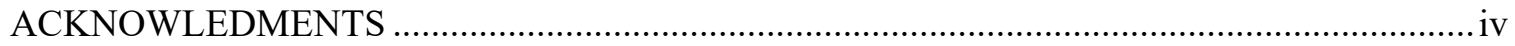

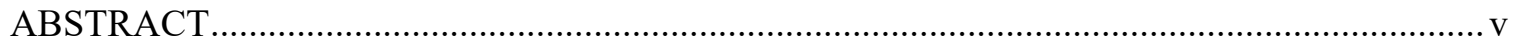

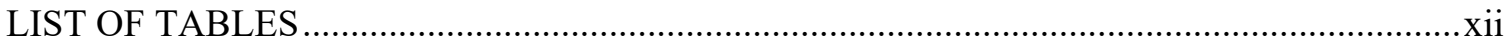

LIST OF FIGURES ……………………………………………………………....

Chapter 1 INTRODUCTION................................................................................. 1

$1.1 \quad$ Surface-Bound Biomolecules ………………………………………………….. 1

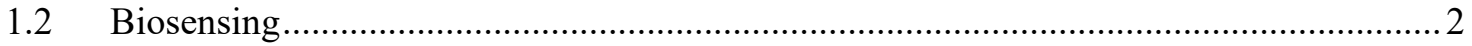

1.3 Surface-Specific and Biosensing Analytical Techniques ................................................. 3

1.4 Surface Plasmon Resonance (SPR) Technique ………………………………………. 6

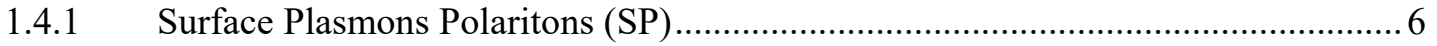

1.4.2 Excitation of Surface Plasmon .........................................................................

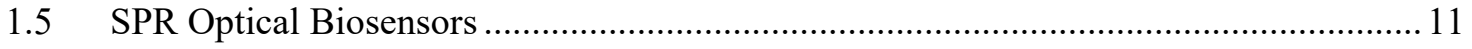

1.6 Electrochemical-Surface Plasmon Resonance Technique (EC-SPR) .............................. 11

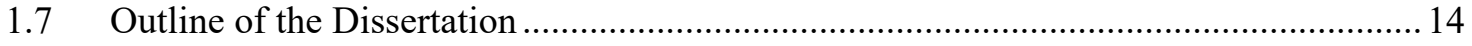

Chapter 2 ELECTROCHEMICAL SURFACE PLASMON RESONANCE (EC-SPR): DESIGN

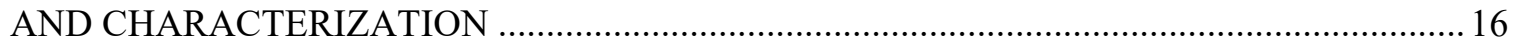

2.1 Conventional Surface Plasmon Resonanse (SPR) ………………………………...... 16

2.1.1 Surface plasmon resonanse platform preparation ………………………………..... 17

2.1.2 Metal Thin Film Deposition........................................................................... 18

2.1.3 Calibrated Deposition Rate ............................................................................ 18

2.1.4 SPR Platform Characterization .................................................................... 22

2.2 Surface Plasmon Resonans Platforms Thicknesses Optimization ................................. 25

2.3 Electrochemical-Surface Plasmon Resonance Device (EC-SPR)..................................2 29

2.3.1 Micro-Electrochemical Flow Cell Fabrication...................................................... 29

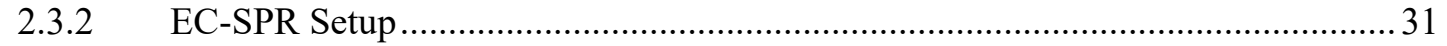

2.3.3 Immobilization and Characterization of Self-Assembled Monolayer onto EC-SPR

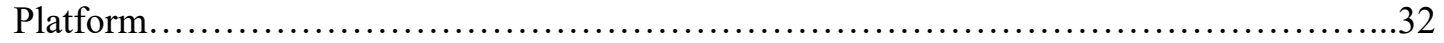

2.3.4 EC-SPR Device Optimization................................................................. 34

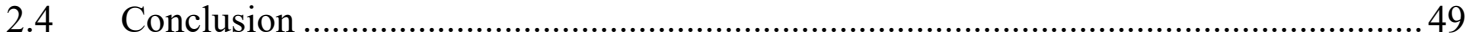

Chapter 3 ELECTRON-TRANSFER KINETICS OF REDOX PROBE AT DIFFERENT

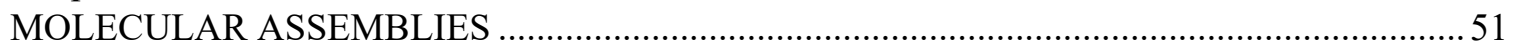

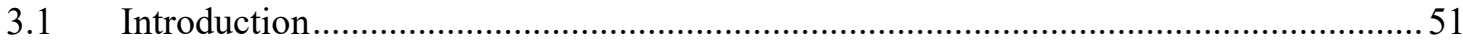


3.2 Theory for the Optical Impedance Spectroscopy Technique (OIS) with the EC-SPR

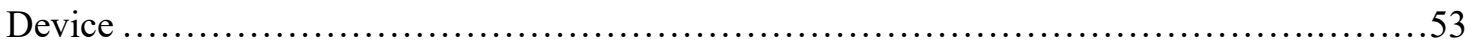

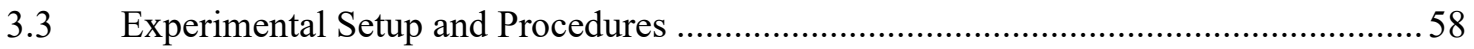

3.4 Functionalization Protocols of the Platform with Different Layers ...............................59

3.4.1 Immobilization of Cytochrome c protein onto MPA-modified EC-SPR Platform 60

3.4.2 Immobilization of Cytochrome C Antibody (Cyt c Ab) onto CYS- modified EC-

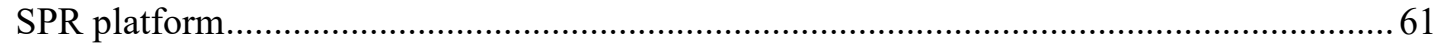

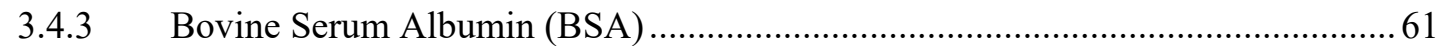

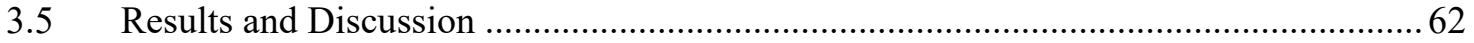

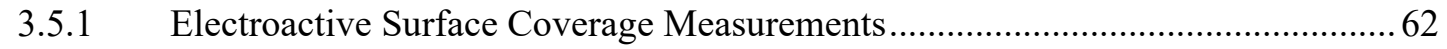

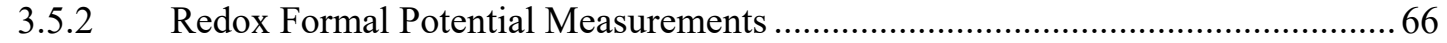

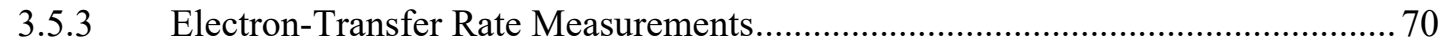

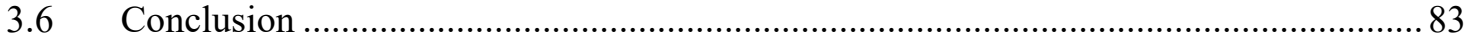

Chapter 4 INFLUENZA VIRUS DETECTION WITH A FUNCTIONALIZED EC-SPR

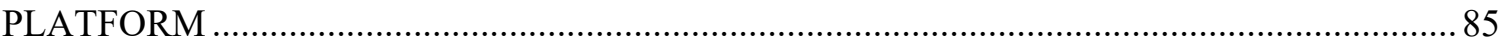

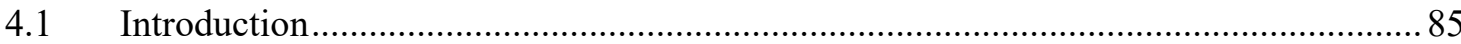

4.2 The EC-SPR Methodology for Detection of Influenza Virus Immunosensing .............87

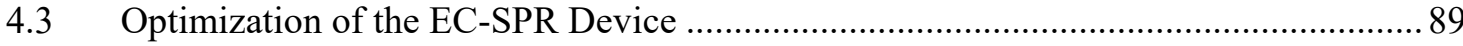

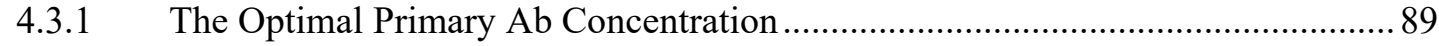

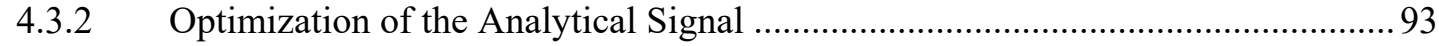

4.4 Virus Protein Detection with Immunoassay on the EC-SPR Device .............................96

4.4.1 Sample Preparation and Functionalization Protocol of the EC-SPR Surface with an Immunoassay Targeting an Influenza Virus Antigen ......................................................... 96

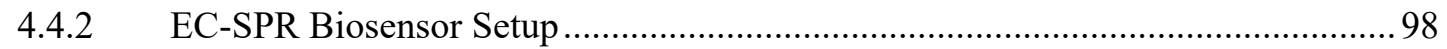

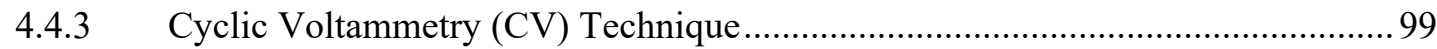

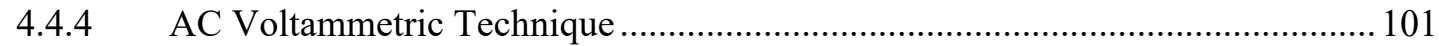

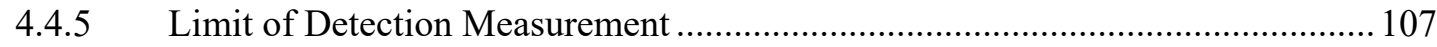

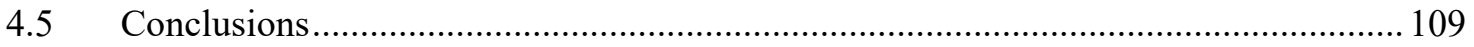

Chapter 5 DETECTING ENDOTOXIN BACTERIA IN BIOLOGICAL SAMPLES................ 111

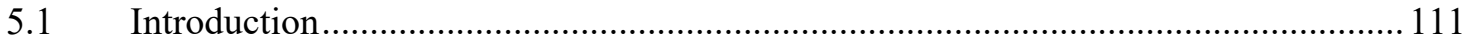

5.2 Gram-Negative Endotoxin Detection with Immunoassay ....................................... 112

5.3 Functionalization Protocol of the EC-SPR Sensor Platform for Endotoxin Detection

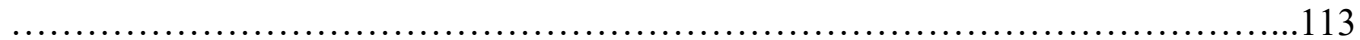

5.4 Endotoxin Detection with Immunoassay on the EC-SPR Device ............................. 114

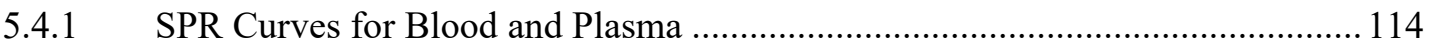


5.4.2 Specific Detection of Endotoxin in PBS Buffer Solution and the Limit of

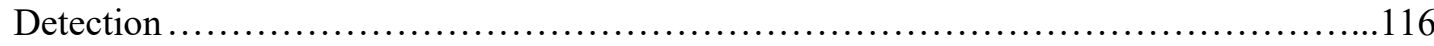

5.4.3 Specific Detection of Endotoxin in Complex Samples (Blood and Plasma) ....... 120

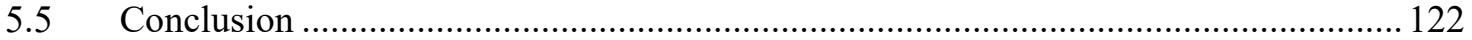

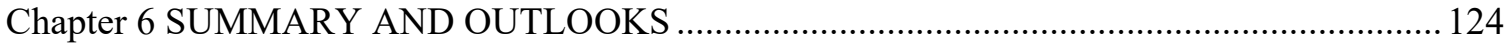

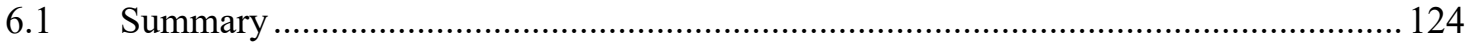

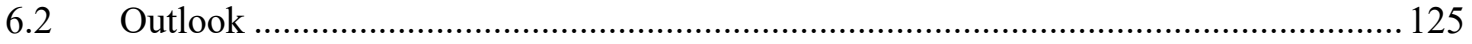

6.2.1 Nanostructured Semiconductors Investigations ................................................ 125

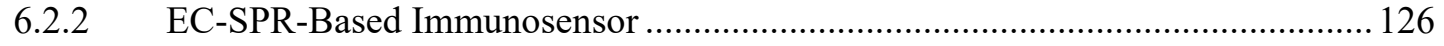

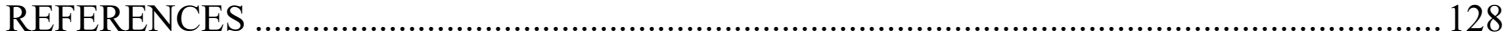

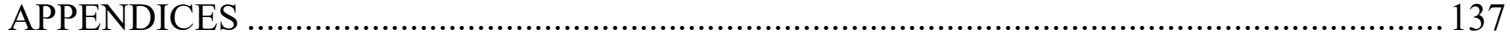

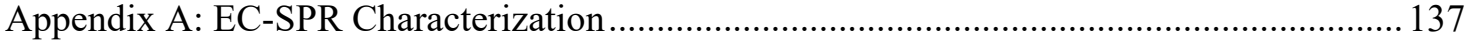

1. Mathematica Program for Obtaining the Optical Constants of A thin Film ..................... 137

2. Calibrated Deposition Rate of Silver Thin Film ........................................................... 142

3. Mathematica Program for the SPR Reflectivity Calculated ........................................... 144

4. Calculated SPR Reflectance Curves for Gold, Silver, and Bilayered (silver/gold) Thin

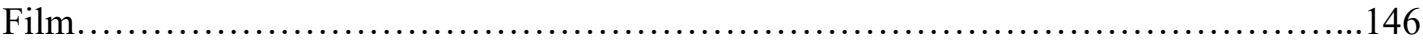

5. Calculated SPR Reflectance Curves Using the Optimal Thickness for Gold, Silver, and Bilayered (silver/gold) Thin Film .... 151

6. Reflectance under a CV Scan for the Cytochrome c protein Immobilized onto MPABilayered (Ag/Au) Surface 151

Appendix B: Electron-Transfer Rate Measurements ............................................................ 155

1. Cytochrome c protein Directly Immobilized onto the EC-SPR Platform......................... 155

2. Cytochrome c protein Absorbed onto MPA-modified EC-SPR Platform ....................... 158

3. Cytochrome c protein Absorbed onto activated MPA-modified EC-SPR Platform ......... 161

4. Cytochrome c protein Absorbed onto Cyt c Ab /CYS-Modified EC-SPR Platform ........ 163

5. Cytochrome c protein Absorbed onto Cyt c Ab /CYS-Modified EC-SPR Platform with the

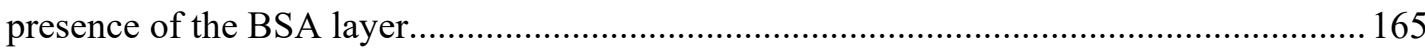

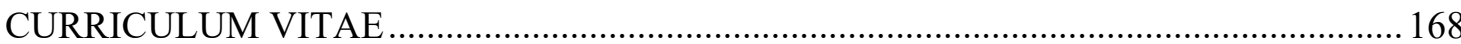




\section{LIST OF TABLES}

TABLE

PAGE

Table 2-1: The optical constant of gold and silver thin films were extracted by minimizing the difference between the measured and calculated SPR reflectance curves. 25

Table 2-2: The optimal thickness of the three configurations (gold, silver, and bilayered [silver/ gold]) platform that demonstrated a strong resonant condition in an aqueous environment.

Table 2-3:Optical constants of cytochrome c protein film were extracted by minimizing the difference between the measured SPR reflectance curves and the calculated curves.

Table 3-1:The electroactive surface coverage of cytochrome c protein adsorbed at each functionalized layer by a cyclic voltammetry $(\mathrm{CV})$ technique. .64

Table 3-2: The electrical and optical measurements of the redox formal potentials for cytochrome c protein redox reactions at the different functionalized layers on the EC-SPR platform. 68 Table 3-3: The electron-transfer rates of cytochrome $\mathrm{c}$ protein for the different immobilization strategies. 


\section{LIST OF FIGURES}

FIGURE

PAGE

Figure 1-1: A schematic representation of the Kretschmann configuration for exciting a surface

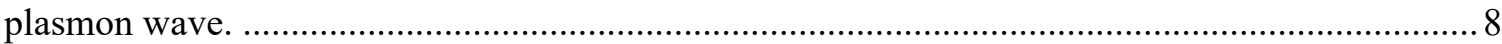

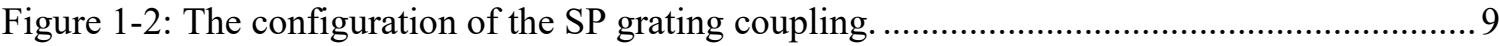
Figure 1-3: Reflectivity profile against the incident angle of a monochromatic p-polarized light.10 Figure 2-1: Diagram of a conventional surface plasmon resonance (SPR). CW laser with combination of a half-wave plate and a linear polarizer to establish linearly polarized light at transverse-magnetic (TM) polarization was used as an excitation light. Prism/metal-coated substrate configuration was set up on a rotation stage to precisely control the incident angle. An index matching gel was used to optically couple the metal-coated substrate to an equilateral BK7 glass prism. A photo-detector was used to collect the reflected light.

Figure 2-2: Measured and calculated SPR reflectance profile to determine the thickness of the gold thin films at three deposition times. The red, blue, and black dots are measured data obtained by the conventional SPR setup at 60 seconds, 70 seconds, and 90 seconds, respectively. The red, blue, and black solid lines are calculated SPR reflectivity curves at 60 seconds, 70 seconds, and 90 seconds, respectively...... 20

Figure 2-3: The measured gold thickness against the deposition time. The deposition rate is

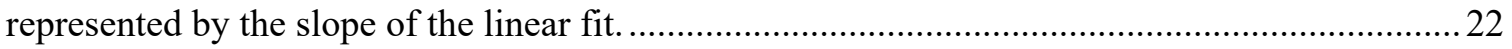
Figure 2-4: The optical constants examination of the gold thin film in air. The dots are measured data obtained by the conventional SPR setup at each excitation wavelength. The solid lines are calculated SPR reflectance curves based on a Mathematica program. The wavelengths of light are $552 \mathrm{~nm}, 637 \mathrm{~nm}, 660 \mathrm{~nm}, 685 \mathrm{~nm}$, and $785 \mathrm{~nm}$.

Figure 2-5: The optical constants examination of the silver thin film in PBS buffer solution. The dots are experimental results obtained by the conventional SPR setup at each excitation wavelength. The solid lines are the calculated SPR reflectance curves. The excitation wavelengths were 552 $\mathrm{nm}, 637 \mathrm{~nm}, 660 \mathrm{~nm}, 685 \mathrm{~nm}$, and $785 \mathrm{~nm}$. 24 Figure 2-6: The calculated SPR reflectance curves for a) gold thin film with thickness of $30 \mathrm{~nm}$, $35 \mathrm{~nm}, 40 \mathrm{~nm}, 45 \mathrm{~nm}$, and $50 \mathrm{~nm}$, b) silver thin film with thickness of $30 \mathrm{~nm}, 35 \mathrm{~nm}, 40 \mathrm{~nm}, 45$ $\mathrm{nm}$, and $50 \mathrm{~nm}$, and c) bilayered (silver/gold) thin film with silver thin film thickness of $30 \mathrm{~nm}, 35$ $\mathrm{nm}, 40 \mathrm{~nm}, 45 \mathrm{~nm}$, and $50 \mathrm{~nm}$ and a fixed thickness of gold thin film $(5 \mathrm{~nm})$. The following values were used for the calculations: $\mathrm{n}_{\text {prism }}=1.51842, \mathrm{n}_{\text {gold }}=0.38559+2.61439 \mathrm{i}, \mathrm{n}_{\text {silver }}=0.0719+3.2431 \mathrm{i}$, and $\mathrm{n}_{\text {Buffer }}=1.32045$. The excitation wavelength was $552 \mathrm{~nm}$.

Figure 2-7: The calculated SPR reflectance curves for silver $(30 \mathrm{~nm})$, gold $(35 \mathrm{~nm})$ and bilayered silver $(35 \mathrm{~nm}) /$ gold $(5 \mathrm{~nm})$ thin films. The following values were used for the calculations: $\mathrm{n}_{\text {prism }}$ $=1.51842, \mathrm{n}_{\text {gold }}=0.38559+2.61439 \mathrm{i}, \mathrm{n}_{\text {silver }}=0.0719+3.2431 \mathrm{i}, \mathrm{n}_{\text {Buffer }}=1.32045$, and the wavelength of light was $552 \mathrm{~nm}$. 28 Figure 2-8: (a) Cross-section of the electrochemical flow cell with liquid inlet and outlet and two gold electrodes. (b) Schematic of the electrochemical flow cell with three-electrodes attached to the prism coupling unit in the Kretschmann configuration and mounted on a rotating stage. A syringe pump was used for handling the liquid injection into the electrochemical flow cell and a plastic tube for the liquid waste. 30 Figure 2-9: Schematic representation of the EC-SPR setup with a potentiostat for electrical control, laser light, polarizer, and half-wave retarder for P polarized light. A detector, current amplifier, lock-in amplifier and oscilloscope were used for data collection. 
Figure 2-10: The optical constants of the MPA layer in PBS buffer solution. The data points are experimental results obtain by the conventional SPR setup at each excitation wavelength. The solid lines are calculated SPR reflectance curves. The wavelengths of light are $(552 \mathrm{~nm}, 637 \mathrm{~nm}, 660$ $\mathrm{nm}, 685 \mathrm{~nm}$, and $785 \mathrm{~nm}$ ).

Figure 2-11: The experimental SPR reflectance curves of PBS buffer solution (red dotes) and assembly of surface-bound cytochrome c protein (blue dotes) using (a) $552 \mathrm{~nm}$, (b) $637 \mathrm{~nm}$, (d) $660 \mathrm{~nm}$, (d) $685 \mathrm{~nm}$, and (e) $785 \mathrm{~nm}$ excitation wavelengths.

Figure 2-12: The optical constants examination of the cytochrome c layer in PBS buffer. The blue dotted curves are the experimental results obtained by the EC-SPR setup at $-0.3 \mathrm{~V}$, while the red dotted curves are at $0.3 \mathrm{~V}$. The blue and red solid curves are the calculated SPR reflectivity curves using six-layer model (KB7/silver/gold/MPA/cytochrome c protein/PBS buffer) at $-0.3 \mathrm{~V}$ and $0.3 \mathrm{~V}$, respectively with (a) $552 \mathrm{~nm}$, (b) $637 \mathrm{~nm}$, (c) $660 \mathrm{~nm}$, (d) $685 \mathrm{~nm}$, and (e) $785 \mathrm{~nm}$ excitation wavelengths.

Figure 2-13: Right y-axis shows the molar absorptivity for the oxidized and the reduced states of cytochrome c protein in PBS buffer solution measured by UV-vis spectrophotometer, while the left $\mathrm{y}$-axis shows the refractive index for cytochrome c protein, $k$, obtained by the SPR reflectance curves.

Figure 2-14: Left y-axis shows the experimental SPR reflectance curves of assembly of surfacebound cytochrome c protein at oxidized and reduced states (the dotted curves) and the calculated data at the same two oxidation states (the line curves), while right y-axis shows the measured (the dotted curve) and the calculated (the line curves) of $\Delta I$, with (a) $552 \mathrm{~nm}$, (b) $637 \mathrm{~nm}$, (c) $660 \mathrm{~nm}$, (d) $685 \mathrm{~nm}$, and (e) $785 \mathrm{~nm}$ excitation wavelengths.

Figure 2-15: $\Delta \mathrm{I}$ against the effective refractive index inside the prism, $n \sin (\theta)$, at $552 \mathrm{~nm}, 637 \mathrm{~nm}$, $660 \mathrm{~nm}, 685 \mathrm{~nm}$ and $785 \mathrm{~nm}$ excitation wavelengths. The dotted curves are the experimental data, whereas the line curves are the calculated data. 44 Figure 2-16: (a) Cyclic voltammetry with cytochrome c protein immobilized onto the MPAbilayered (silver/gold) platform with scan rate of $0.02 \mathrm{~V} / \mathrm{s}$. (b) The redox peak area. .................. 46 Figure 2-17: Reflectance under a CV scan for the cytochrome c protein immobilized onto MPAbilayered (silver/gold) platform with scan rate of $0.02 \mathrm{~V} / \mathrm{s}$ and at three values of $n \sin (\theta) \ldots \ldots \ldots . .47$ Figure 2-18: Reflectance under a CV scan for the cytochrome c protein immobilized onto MPAbilayered (silver/gold) platform with scan rate of $0.02 \mathrm{~V} / \mathrm{s}$ and using the optimal $n \sin (\theta)$ at each wavelength.

Figure 3-1: Generalized equivalent electrical circuit for the electrochemical flow cell for molecules assemblies. 56

Figure 3-2: Schematic diagram of EC-SPR setup with a potentiostat for electrical control, laser light, polarizer, and half-wave retarder for P-polarized light. A detector, current amplifier, lock-in amplifier, and oscilloscope were used for data collection. .58 Figure 3-3: The electrical current response under the CV scan for cytochrome c protein in $10 \mathrm{mM}$ PBS buffer solution at scan rate of $0.02 \mathrm{~V} / \mathrm{s}$. Black curve represents the direct adsorption, orange curve represents the electrostatic adsorption, blue cure represents the covalent adsorption, red curve represents the affinity adsorption, and green curve represents the affinity adsorption with the presence of the BSA.

Figure 3-4: Gaussian fitting of the reduction peaks for cytochrome c protein assemblies. Black curve represents the direct adsorption, orange curve represents the electrostatic adsorption, blue cure represents the covalent adsorption, red curve represents the affinity adsorption, and green curve represents the affinity adsorption with the presence of the BSA. 64 
Figure 3-5: SPR optical signal, $\Delta \mathrm{I}_{\mathrm{SPR}}$, versus the DC bias potential ranging from (-0.3 V to $\left.0.35 \mathrm{~V}\right)$ measured under a sinusoidal potential modulation with an amplitude modulation of $20 \mathrm{mV}$ at the frequencies of (a) $50 \mathrm{~Hz}$, (b) $150 \mathrm{~Hz}$, (c) $150 \mathrm{~Hz}$, (d) $250 \mathrm{~Hz}$, and (e) $150 \mathrm{~Hz}$......................... 68 Figure 3-6: SPR optical signal, $\triangle I_{S P R}$, measured under $20 \mathrm{mV}$ AC amplitude of impedance potential modulations at the DC bias potential of $0.015 \mathrm{~V}$ is plotted against angular modulation frequency

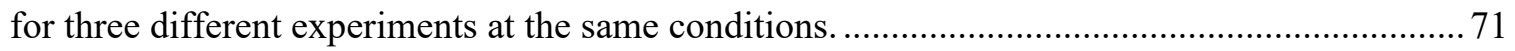
Figure 3-7: (a) The real components of the total electrical impedance results of the electrochemical flow cell for cytochrome c protein directly absorbed onto EC-SPR platform, where (b) represents

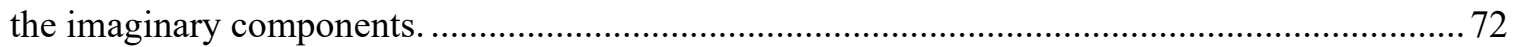
Figure 3-8: Electron-transfer rate calculation. The electron-transfer rate is represented by the slope of the linear fit. 73 Figure 3-9: SPR optical signal, $\triangle I_{S P R}$, measured under $20 \mathrm{mV}$ AC amplitude of impedance potential modulations at the formal potential is plotted against angular modulation frequency for (a) electrostatic immobilization, and (b) covalent immobilization.

Figure 3-10: Electrical impedance results of the electrochemical flow cell. (a) Presents the real component of the total electrical impedance for electrostatic adsorption, whereas (b) the imaginary component. (c) Presents the real component of the total electrical impedance for covalent adsorption, whereas (d) the imaginary component. 76

Figure 3-11: Electron-transfer rate calculation. The electron-transfer rate is represented by the slope of the linear fit, (a) for electrostatic adsorption, whereas (b) for covalent adsorption................... 77 Figure 3-12: SPR optical signal, $\triangle I_{S P R}$, measured under $20 \mathrm{mV}$ AC amplitude of impedance potential modulations is plotted against angular modulation frequency for three different experiments at the same conditions, (a) without the BSA layer, whereas (b) with the presence of the BSA layer...... 78 Figure 3-13: The real component of the total electrical impedance of the electrochemical flow cell (a) with cytochrome $\mathrm{c}$ protein captured by Cyt $\mathrm{c} \mathrm{Ab}$, while (b) is the imaginary component. (c) The real component after adding the BSA layer, while $(\mathrm{d})$ is the imaginary component...................... 80 Figure 3-14: Electron-transfer rate calculation. The electron-transfer rate is represented by the slope of the linear fit, (a) without BSA layer, whereas (b) with BSA layer......................................... 80 Figure 4-1: A glass slide coated with a $5 \mathrm{~nm}$ chromium adhesion layer followed by a $50 \mathrm{~nm}$ layer of gold to create a biosensor surface. A molecular assembly formed by layers of MPA, EDC, and NHS were used to functionalize the biosensor interface. 88 Figure 4-2: The SPR reflectance curves against the effective refractive index inside the prism for a PBS buffer solution and three different concentrations of capturing Ab................................... 90 Figure 4-3: Cyclic voltammograms for three different concentrations of MB-labeled secondary Ab

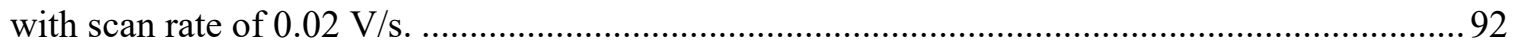
Figure 4-4: SPR reflectance for the PBS buffer and MB solutions at $-0.35 \mathrm{~V}$ and $0.35 \mathrm{~V}$........... 94 Figure 4-5: Left y-axis shows the SPR reflectance for the PBS buffer solution, while the right $y-$ axis shows the difference in the optical signal $\Delta \mathrm{I}$ between the oxidized state and the reduced state of the MB molecule. 95

Figure 4-6: Schematic diagram of the monoclonal anti-H5 (H5N1) capture Ab immobilization on the gold surface modified with MPA molecules and activated with EDC/NHS.........................97 Figure 4-7:Schematic diagram of EC-SPR setup with a potentiostat for electrical control, laser light, polarizer and half-wave retarder for $\mathrm{P}$ polarized light. A detector, current amplifier, lock-in amplifier and oscilloscope were used for data collection. ......................................................... 98 Figure 4-8: Optical signal, I, under a CV scan for the full immunoassay sandwich at scan rate of $0.02 \mathrm{~V} / \mathrm{s}$. The red trace shows the data in the absence of HA virus antigen, while the black trace represents the data in the presence of HA virus antigen. 100 
Figure 4-9: Optical signal, I, under a CV scan for the MB-labeled polyclonal Ab adsorbed onto the activated MPA at scan rate of $0.02 \mathrm{~V} / \mathrm{s}$.

Figure 4-10: SPR optical signal, $\triangle \mathrm{ISPR}$, measured under AC potential modulation with the MBlabeled polyclonal $\mathrm{Ab}$ adsorbed onto the activated MPA-modified sensor surface at the DC bias potential of $-0.25 \mathrm{~V}$.

Figure 4-11: Optical signal, I, under a CV scan for the full immunoassay sandwich at scan rate of $0.02 \mathrm{~V} / \mathrm{s}$. The blue trace shows the fitting of the optical signal, while the red trace represents the derivative of the fitting of the optical signal. 104 Figure 4-12:SPR optical signal, $\triangle \mathrm{ISPR}$, measured under AC potential modulation with the full immunoassay sandwich at the DC bias potential of $-0.14 \mathrm{~V}$ and a HA virus antigen concentration of $200 \mathrm{ng} / \mathrm{ml}$. 105 Figure 4-13: SPR optical signal, $\Delta \mathrm{I}_{\mathrm{SPR}}$, versus the DC bias potential with (a) the full immunoassay sandwich, (b) the MB-labeled polyclonal Ab adsorbed onto the MPA measured under a sinusoidal potential modulation $(0.04 \mathrm{~V})$.

Figure 4-14: SPR optical signal, $\Delta \mathrm{I}_{\mathrm{SPR}}$, versus the DC bias potential in the absence of HA virus antigen and for different concentrations of the HA virus antigen measured under a sinusoidal potential modulation at the frequency of $250 \mathrm{~Hz}$ and an amplitude modulation of $0.04 \mathrm{~V}$.

Figure 4-15: Calibration curve based on AC measurements for detection of different concentrations of the HA virus antigen.

Figure 5-1: The reflectance against the time measured by the SPR technique at a fixed incident angle for a monoclonal anti-HA (H5N1) capture Ab adsorbed covalently onto MPA-modified sensor platform using EDC/NHS coupling chemistry.

Figure 5-2: The SPR reflectance curves against the effective refractive index inside the prism for a PBS buffer solution (blue dots), plasma (yellow dots), and blood (red dots) samples............... 116 Figure 5-3: SPR optical signal, $\Delta \mathrm{I}_{\mathrm{SPR}}$, measured under AC potential modulation at the $\mathrm{DC}$ bias potential of $-0.2 \mathrm{~V}$ in the presence of the $0.05 \mathrm{EU} / \mathrm{ml}$ endotoxin in the flow cell (red curve), and in the absence of the endotoxin in the flow cell (blue curve). 117 Figure 5-4: SPR optical signal, $\Delta \mathrm{I}_{\mathrm{SPR}}$, versus the $\mathrm{DC}$ bias potential in the absence of endotoxin (blue curve) and for $0.05 \mathrm{EU} / \mathrm{ml}$ endotoxin present in PBS buffer solution (red curve) measured under a sinusoidal potential modulation at the frequency of $1 \mathrm{kHz}$ and an amplitude modulation of $0.04 \mathrm{~V}$.

Figure 5-5: SPR optical signal, $\triangle I_{S P R}$, versus the DC bias potential in the absence of endotoxin and for different concentrations of the endotoxin measured under a sinusoidal potential modulation at the frequency of $1 \mathrm{KHz}$ and an amplitude modulation of $0.04 \mathrm{~V}$. 119

Figure 5-6: Calibration curve based on AC measurements for detection of different concentrations of the endotoxin. 120 Figure 5-7: (a) SPR optical signal, $\triangle \mathrm{I}_{\mathrm{SPR}}$, measured under AC potential modulation at the DC bias potential of $-0.05 \mathrm{~V}$, red curve with $0.05 \mathrm{EU} / \mathrm{ml}$ endotoxin spiked on blood, blue curve without endotoxin. (b) SPR optical signal, $\Delta \mathrm{I}_{\mathrm{SPR}}$, versus the DC bias potential, red curve with $0.05 \mathrm{EU} / \mathrm{ml}$ endotoxin spiked on plasma, blue curve without Endotoxin.

Figure 5-8: SPR optical signal, $\triangle \mathrm{I}_{\mathrm{SPR}}$, versus the DC bias potential, (a) red curve with $0.05 \mathrm{EU} / \mathrm{ml}$ endotoxin spiked in blood, blue curve without endotoxin, (b) red curve with $0.05 \mathrm{EU} / \mathrm{ml}$ endotoxin spiked in plasma, blue curve without Endotoxin.

Figure A-1: Measured and calculated SPR reflectance profile to determine the thickness of the silver thin films at three deposition times. The blue, black, and red dots are measured data obtained by the conventional SPR setup at 50 seconds, 40 seconds, and 30 seconds, respectively. The blue, black, and red solid lines are calculated SPR reflectivity curves at 50 seconds, 40 seconds, and 30 
seconds, respectively.

Figure A-2: The measured silver thickness against the deposition time. The deposition rate is represented by the slope of the linear fit.

140

Figure A-3: The calculated SPR reflectance curves for (a) gold thin film, (b) silver thin film with thickness of $30 \mathrm{~nm}, 35 \mathrm{~nm}, 40 \mathrm{~nm}, 45 \mathrm{~nm}$, and $50 \mathrm{~nm}$, and (c) bilayered (silver/gold) thin film with silver thin film thickness of $30 \mathrm{~nm}, 35 \mathrm{~nm}, 40 \mathrm{~nm}, 45 \mathrm{~nm}$, and $50 \mathrm{~nm}$ and a fixed thickness of gold thin film $(5 \mathrm{~nm})$. The following values were used for the calculations: $\mathrm{n}_{\text {prism }}=1.51842, \mathrm{n}_{\text {gold }}=$ $0.38559+2.61439 \mathrm{i}, \mathrm{n}_{\text {silver }}=0.0719+3.2431 \mathrm{i}$, and $\mathrm{n}_{\text {Buffer }}=1.32045$. The excitation wavelength is $637 \mathrm{~nm}$.

Figure A-4: The calculated SPR reflectance curves for (a) gold thin film, (b) silver thin film with thickness of $30 \mathrm{~nm}, 35 \mathrm{~nm}, 40 \mathrm{~nm}, 45 \mathrm{~nm}$, and $50 \mathrm{~nm}$, and (c) bilayered (silver/gold) thin film with silver thin film thickness of $30 \mathrm{~nm}, 35 \mathrm{~nm}, 40 \mathrm{~nm}, 45 \mathrm{~nm}$, and $50 \mathrm{~nm}$ and a fixed thickness of gold thin film $(5 \mathrm{~nm})$. The following values were used for the calculations: $\mathrm{n}_{\text {prism }}=1.51842, \mathrm{n}_{\text {gold }}=$ $0.38559+2.61439 \mathrm{i}, \mathrm{n}_{\text {silver }}=0.0719+3.2431 \mathrm{i}$, and $\mathrm{n}_{\text {Buffer }}=1.32045$. The excitation wavelength is $660 \mathrm{~nm}$. 144

Figure A-5: The calculated SPR reflectance curves for (a) gold thin film, (b) silver thin film with thickness of $30 \mathrm{~nm}, 35 \mathrm{~nm}, 40 \mathrm{~nm}, 45 \mathrm{~nm}$, and $50 \mathrm{~nm}$, and (c) bilayered (silver/gold) thin film with silver thin film thickness of $30 \mathrm{~nm}, 35 \mathrm{~nm}, 40 \mathrm{~nm}, 45 \mathrm{~nm}$, and $50 \mathrm{~nm}$ and a fixed thickness of gold thin film $(5 \mathrm{~nm})$. The following values were used for the calculations: $\mathrm{n}_{\text {prism }}=1.51842, \mathrm{n}_{\text {gold }}=$ $0.38559+2.61439 \mathrm{i}, \mathrm{n}_{\text {silver }}=0.0719+3.2431 \mathrm{i}$, and $\mathrm{n}_{\text {Buffer }}=1.32045$. The excitation wavelength is $685 \mathrm{~nm}$.

Figure A-6: The calculated SPR reflectance curves for (a) gold thin film, (b) silver thin film with thickness of $30 \mathrm{~nm}, 35 \mathrm{~nm}, 40 \mathrm{~nm}, 45 \mathrm{~nm}$, and $50 \mathrm{~nm}$, and (c) bilayered (silver/gold) thin film with silver thin film thickness of $30 \mathrm{~nm}, 35 \mathrm{~nm}, 40 \mathrm{~nm}, 45 \mathrm{~nm}$, and $50 \mathrm{~nm}$ and a fixed thickness of gold thin film $(5 \mathrm{~nm})$. The following values were used for the calculations: $\mathrm{n}_{\text {prism }}=1.51842, \mathrm{n}_{\text {gold }}=$ $0.38559+2.61439 \mathrm{i}, \mathrm{n}_{\text {silver }}=0.0719+3.2431 \mathrm{i}$, and $\mathrm{n}_{\text {Buffer }}=1.32045$. The excitation wavelength is

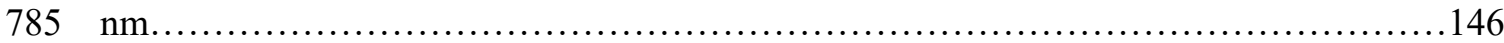

Figure A-7: The calculated SPR reflectance curves using the optimal thickness for the three configurations at (a) $637 \mathrm{~nm}$, (b) $660 \mathrm{~nm}$, (d) $685 \mathrm{~nm}$, and (d) $785 \mathrm{~nm}$. The following values were used for the calculations: $\mathrm{n}_{\text {prism }}=1.51842, \mathrm{n}_{\text {gold }}=0.38559+2.61439 \mathrm{i}, \mathrm{n}_{\text {silver }}=0.0719+3.2431 \mathrm{i}, \mathrm{n}_{\text {Buffer }}$ $=1.32045$.

Figure A-8: Reflectance under a CV scan for the cytochrome c protein immobilized onto MPAbilayered (silver/gold) platform with scan rate of $0.02 \mathrm{~V} / \mathrm{s}$ and at three values of $n \sin (\theta)$ for $637 \mathrm{~nm}$

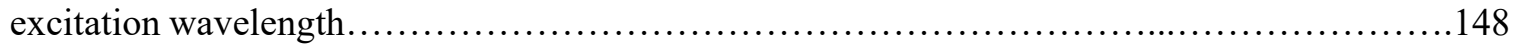
Figure A-9: Reflectance under a CV scan for the cytochrome c protein immobilized onto MPAbilayered (silver/gold) platform with scan rate of $0.02 \mathrm{~V} / \mathrm{s}$ and at three values of $\mathrm{n} \sin (\theta)$ for $660 \mathrm{~nm}$ excitation wavelength............................................................. 149 Figure A-10: Reflectance under a CV scan for the cytochrome c protein immobilized onto MPAbilayered (silver/gold) platform with scan rate of $0.02 \mathrm{~V} / \mathrm{s}$ and at three values of $n \sin (\theta)$ for $685 \mathrm{~nm}$

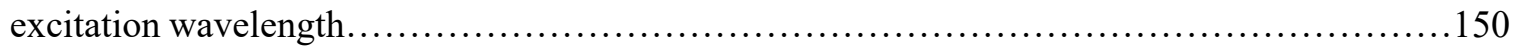
Figure A-11: Reflectance under a CV scan for the cytochrome c protein immobilized onto MPAbilayered (silver/gold) platform with scan rate of $0.02 \mathrm{~V} / \mathrm{s}$ and at three values of $\mathrm{n} \sin (\theta)$ for $785 \mathrm{~nm}$ excitation wavelength. 151 Figure B-1: SPR optical signal, $\triangle \mathrm{I} S P R$, measured under $20 \mathrm{mV}$ AC amplitude of impedance potential modulations at the DC bias potential of $0.094 \mathrm{~V}$ is plotted against angular modulation frequency for two different experiments at the same conditions. 152 
Figure B-2: (a) and (c), represent the real components of the total electrical impedance of the electrochemical flow cell for cytochrome c protein directly absorbed onto EC-SPR platform, while

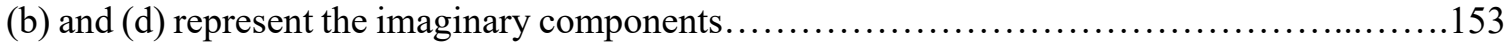

Figure B-3: Electron-transfer rate calculation. The electron-transfer rate is represented by the slope

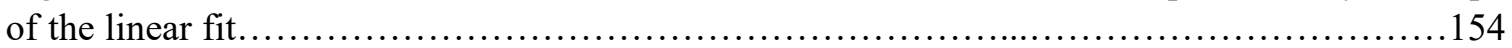
Figure B-4: SPR optical signal, $\triangle I_{S P R}$, measured under $20 \mathrm{mV}$ AC amplitude of impedance potential modulations at the DC bias potential of $0.011 \mathrm{~V}$ is plotted against angular modulation frequency for two different experiments at the same conditions. 155 Figure B-5: (a) and (c), represent the real components of the total electrical impedance of the electrochemical flow cell for cytochrome c protein electrostatically absorbed onto MPA modified EC-SPR platform, while (b) and (d) represent the imaginary components.................156 Figure B-6: Electron-transfer rate calculation. The electron-transfer rate is represented by the slope of the linear fit 156 Figure B-7: SPR optical signal, $\triangle I_{S P R}$, measured under $20 \mathrm{mV}$ AC amplitude of impedance potential modulations at the DC bias potential of $0.0114 \mathrm{~V}$ is plotted against angular modulation frequency for two different experiments at the same conditions. 157 Figure B-8: (a) and (c), represent the real components of the total electrical impedance of the electrochemical flow cell for cytochrome c protein covalently absorbed onto MPA modified ECSPR platform, while (b) and (d) represent the imaginary components...................... 158 Figure B-9: Electron-transfer rate calculation. The electron-transfer rate is represented by the slope of the linear fit.... 159

Figure B-10: SPR optical signal, $\triangle \mathrm{I}_{\mathrm{SPR}}$, measured under $20 \mathrm{mV}$ AC amplitude of impedance potential modulations is plotted against angular modulation frequency for two different experiments at the sameconditions. .160 Figure B-11: (a) and (c), represent the real components of the total electrical impedance of the electrochemical flow cell with cytochrome c protein captured by Cyt c Ab, while (b) and (d) represent the imaginary components. 


\section{CHAPTER 1}

\section{INTRODUCTION}

\subsection{Surface-Bound Biomolecules}

Currently, the preparation and characterization of substrate-supported molecular assemblies is a very active area of research worldwide, due to the immense potential for use of biomolecular films in device technologies such as chemical sensors, energy storage/conversion devices, medical implants, and separation media [1-3]. It is well recognized that the functional characteristics of organic molecules deposited as a thin film on a solid substrate are strongly dependent on properties such as molecular orientation, conformation, packing density, and defect density, as well as the physical and chemical properties of the substrate surface $[4,5]$. Developing a systematic understanding of these relationships is considered to be a prerequisite to the rational and effective utilization of thin organic films in molecular device technologies. However, despite considerable research efforts over the past twenty years, characterizing relationships between structure and function in molecular assembly studies, biomolecular thin films remains a technically difficult challenge. The major challenge for molecular assembly studies is the weak signals due to the extremely low concentration of the bimolecular at the surfaces. Advancing the state of knowledge in this field, therefore, requires concurrent development of new analytical capabilities, since existing techniques are in many cases inadequate for in-situ, detailed structural-functional characterization of molecular films. 


\subsection{Biosensing Technologies}

Rapid and accurate detection of human pathogens in clinical samples, such as viruses, bacteria, parasites, and fungi are crucial for allowing faster decision-making on treatment or to accurately assess the danger to the patients. Moreover, accurate diagnosis leads to the institution of therapies that target a narrow spectrum of pathogens instead of empiric therapy aimed at unknown etiologies that destroy a wide variety of commensal organisms as well as the pathogen. The current rapid tests of human pathogens have extremely low sensitivities, making them of limited use in the clinical setting. Tests with higher sensitivity (e.g., bacterial culture) require much longer turn-around times limiting their clinical utility [6]. Highly sensitive rapid tests (e.g. Reverse Transcriptase Polymerase Chain Reaction) are predominantly performed at centralized laboratories and require skilled personnel to run the appropriate tests and interpret results, making it not widely available and very expensive $[7,8]$. The importance of a rapid and accurate detection of pathogens from clinical samples requires developing novel diagnostic tests with I) high sensitivity and superior selectivity, II) easily obtained specimens, and III) easy analysis with low human error. In recent years, biossensing technology has been using successfully as tools that support biological and clinical diagnostics to detect human pathogenic in a rapid and cost-effective manner. Biosensors are a scientific instrument that consist of a biological recognition element (referred as a bio-receptor) and a transduction platform (referred as a transducer) in order to detect and possibly quantify an analyte (referred as a target) $[9,10]$. The biomolecule is often a nucleic acid, an antibody or an enzyme, that is immobilized on a transducer surface [11]. Three key steps play an important role in the construction of a sensitive and specific device for pathogen detection: I) the use of proper 
surfaces, II) the immobilization technique that offers an efficiently and uniformly print the capture probe (antibody, binding proteins, aptamers), and III) the signal detection system [4]. The principle of the biosensor is based on formation of bio-complexes on a transducer surface, then, real-time monitoring of affinity interactions between immobilized biological components and target molecules, and finally convert the biological recognition reaction into a measurable electronic signal.

\subsection{Surface-Specific and Biosensing Analytical Techniques}

Advancement in the fields of studying biomaterials and monitoring of molecular interactions at solid/liquid interfaces provides the tools to develop and improve of transducer technology for human pathogenic sensor applications. Many surface analytical techniques have been applied to the study of biomaterials at solid/liquid interfaces, but most of them still lack sensitivity to interrogate molecular assemblies at low surface concentrations. Scanning probe microscopy (SPM), attenuated total reflectance infrared spectroscopy (ATR-IR), spectral ellipsometry, and surface plasmon resonance (SPR) are four widely used techniques that can offer the possibility to study biomaterials surface interactions. In addition, they have been increasingly applied in the detection of chemical and biological substances in important areas such as medical diagnostics, environmental monitoring, food safety and security. SPM have a dramatic impact upon research in the biological sciences. The technique has the ability to investigate the biomolecular structure, monitor of real-time biological processes and characterize of biomolecular events at interfaces [12-15]. ATR-IR is capable of studying conformational changes in biological macromolecules at a range of solid/aqueous interfaces, although its relative lack of surface sensitivity (1-5 $\mu \mathrm{m}$ of sampling depth) and difficulties in sample preparation are a limit to 
its successful use. Spectral ellipsometry allows the determination of thickness and refractive index of the adsorbed layer $[16,17]$. Therefore, it is possible to quantify the amount of biological material adsorbed on substrates in bulk phases [18-20]. However, this method is very time-consuming, so that only steady-state systems can be examined. SPR technique provides superb sensitivity (100 pg/ $\mathrm{cm}^{2}$ at extremely well-controlled conditions) and has been widely used for probing molecular-surface interactions [21]. However, a limiting factor for performance has been the subtle refractive index changes of a redox adsorbate under interrogation in cases of a miniscule amount of mass deposited onto a sensor surface. Other analytical tools that have been widely disseminated is the electrochemical techniques, which provide for measurements of small changes in either current or interfacial potential as a function of changes in redox state of a surface bound proteins [22-26]. In recent years, the electrochemical sensors have been reported in important applications for detection of saliva amylase [25], genetic mismatch [26], and DNA-drug interactions [25, 27]. More specifically, electrochemical impedance spectroscopy (EIS) approaches are gaining widespread applications in biotechnology [27], tissue engineering [28], and characterization of biological cells [29], disease diagnosis [30, 31], and cell culture monitoring. Faradaic processes in EIS is a label- and mediator-free strategy for rapid screening of biocompatible surfaces, monitoring pathogenic bacteria, and the analysis of heterogeneous systems, especially biological cells and tissues. The successful coupling of electrochemical transduction schemes with the redox fingerprints of naturally existing and complex biomarkers is expected to generate novel, real-time molecular recognition technologies that are highly selective [26-29]. Nonetheless, those technologies only probe mass changes to the interfacial region and are non-specific in their 
response to changes in concentration, dynamic state, or conformation of the bound protein. However, the conventional electrochemical approaches based on electrical signals alone are limited by non-faradaic and non-specific detection and, therefore, remain inadequate for critical physiological applications.

Spectroelectrochemical techniques are the basis of many approached methods for investigating biological systems in bulk phases and have been extended to the characterization of surface-confined biomolecules by spectroscopically monitoring the absorbance/fluorescence changes of a redox couple under potential modulation [32, 33]. These methods, including electro-reflectance [34, 35], transmission impedance [36, 37], and modulated attenuated total reflectance (PM-ATR) $[38,39]$ provide an important route to monitor electron-transfer processes from redox adsorbates by avoiding non-faradaic components that usually obstruct conventional electrochemical approaches based on electrical signals alone and uniquely track the redox event. The optical response (amplitude and phase delay) measured against several modulation frequencies allows the optical reconstruction of key features of the electrochemical process, including the faradaic current and the associated electron-transfer rate. Although those optical impedance techniques represent an important improvement over conventional electrochemical approaches, the optical sensitivity has been a significant limitation to probe redox species with weak optical transitions and/or at low surface concentrations. A significant limitation is because of the small amount of material present at an interface, which typically is in the monolayer or sub-monolayer range. However, a sensitivity enhancement of the analytical signal is always needed for any reliable absorbance measurement in biological assemblies at the monolayer or sub-monolayer levels. 


\subsection{Surface Plasmon Resonance (SPR) Technique}

Surface plasmon resonance (SPR) technique is a surface-sensitive optical method for characterizing and studying organic [40-42] and inorganic ultrathin films [43, 44] at solid/liquid interfaces. The measurements are based on detecting the change in the refractive index of the medium in contact with the SPR surface due to an accumulation of surface-bound mass. SPR has shown a very high sensitivity to changes in refractive index, therefore it has become an important optical technique for a variety of applications, in the fields of chemistry, biochemistry and biology [45].

\subsubsection{Surface Plasmons Resonance (SPR)}

Surface plasmons are surface electromagnetic waves that are dictated by the electromagnetic properties of a metal with a negative dielectric constant, typically gold or silver, and a dielectric medium interface with a positive dielectric constant. The surface plasmons waves are longitudinal waves propagate along the interface of the two mediums due to the collective oscillations of free electrons on the metal. The dispersion relation for the SP at the metal/dielectric interface can be obtained by solving Maxwell's equations in each medium, and the associated boundary conditions. The dispersion relation of the SPR is given by Equation 1-1 [46].

$$
K_{S P R}=\frac{\omega}{c} \sqrt{\frac{\epsilon_{m} \epsilon_{d}}{\epsilon_{m}+\epsilon_{d}}}
$$

Equation 1-1

Where $K_{S P R}$ represent the propagation constant of surface plasmon wave, $c$ is the speed of light in vacuum, $\frac{\omega}{c}$ represent the wave vector of light in free space, and $\epsilon_{m}, \epsilon_{d}$ are the dielectric constants of metal and dielectric medium, respectively. 


\subsubsection{Excitation of Surface Plasmon}

In order to excite the surface plasmon, the $x$-axis component of the incident light, $k_{x}$, should match the propagation constant of surface plasmon wave, $K_{S P R}$. At a given photon energy, as the dielectric constants of metal is negative and the dielectric constants of dielectric medium is positive, the propagation constant of surface plasmon, $K_{S P R}$, is greater than that of the light wave in dielectric medium, $k_{x}$. Hence, in order to excite the SPR, the incident wave-vector must be increased. Two optical popular methods used to increase the incident wave-vector and then excite the surface plasmon: the attenuated total reflection (ATR) and grating coupling.

\subsubsection{ATR Coupling}

The ATR coupling [47] is the most common way used to excite the surface plasmon wave in SPR-based applications. There are two ATR methods: The Otto configuration [48]and the Kretschmann configuration [49]. Both methods have the same principle with different system design. In these method, a prism with high dielectric constant is used. The propagation constant of the light that goes through a prism with a dielectric constant, $\varepsilon_{p}$, is

given by: $K_{x}=\frac{\omega}{c} \sqrt{\epsilon_{p}} \sin (\theta)$

Equation 1-2

Where $\epsilon_{\mathrm{p}}$ is the dispersion of the prism and $\theta$ the internal angle of incidence. Therefore, by increasing the incident angle, the wave-vector of the incident light in the propagation direction, $K_{x}$,will increase and hence this can be made equal to propagation constant of the surface plasmon, as seen in Equation 1-3 [49].

$$
k_{x}=K_{S P R}=\frac{\omega}{c} \sqrt{\epsilon_{\mathrm{p}}} \sin \left(\theta_{S P R}\right)
$$

Equation 1-3 
At the resonance angle, $\theta_{S P R}$, the energy and the momentum of incident light are transfer to excite the surface plasmon wave. The optical electrical filed, $E_{z}$, that associated to the surface plasmon wave is maximum at the interface and exponentially decay in both medium. Figure 1-1 shows the schematic diagram of a SPR-based Kretschmann configuration in which the surface plasmon wave is excited by a monochromatic ppolarized light.

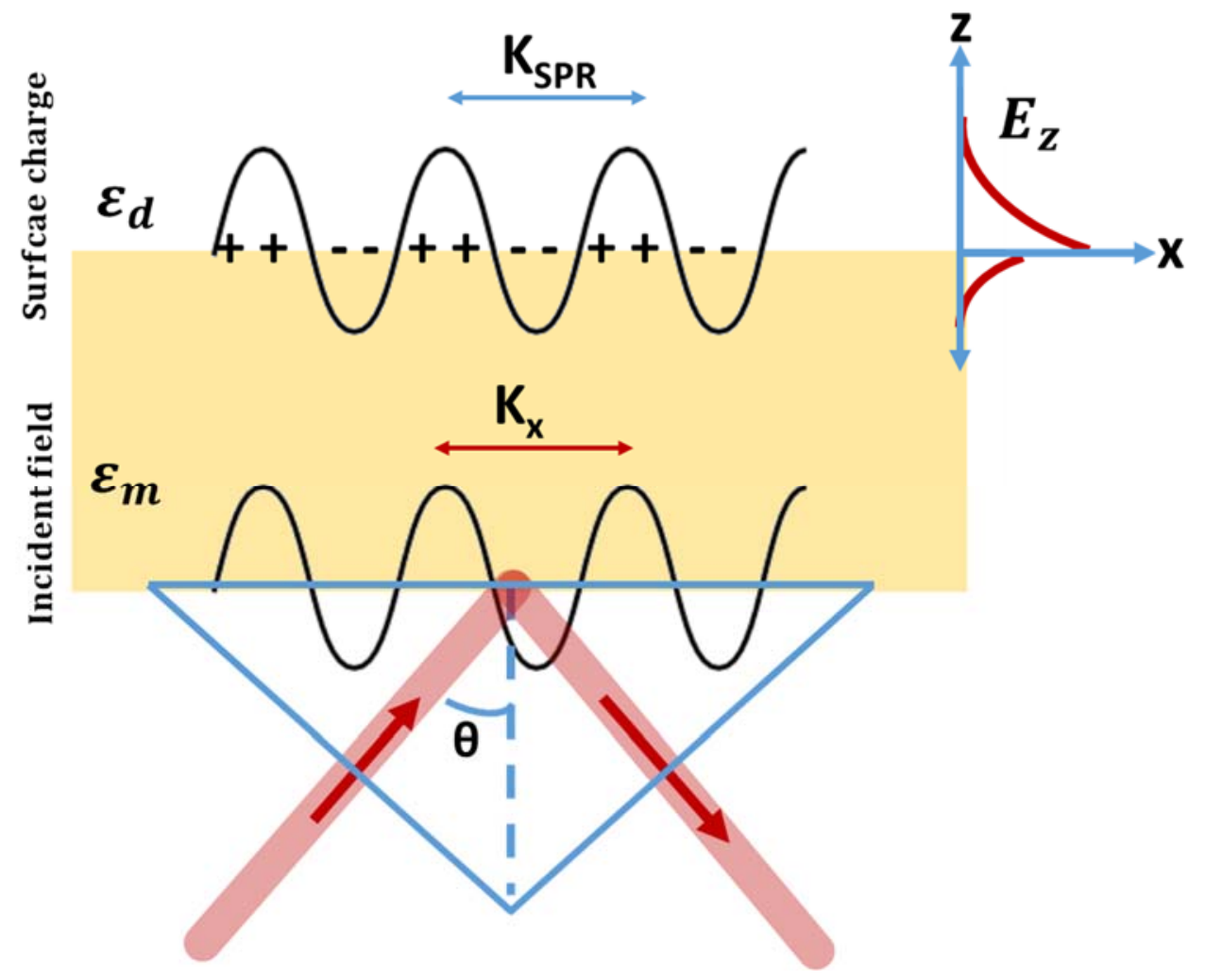

Figure 1-1: A schematic representation of the Kretschmann configuration for exciting a surface plasmon wave. 


\subsubsection{Grating Coupling}

In the grating coupling based-SPR excitation, a light wave is incident at an angle $\theta$ from air onto a metallic grating with a grating constant $a$. Figure 1-2 is illustrated the grating coupling of surface plasmons.

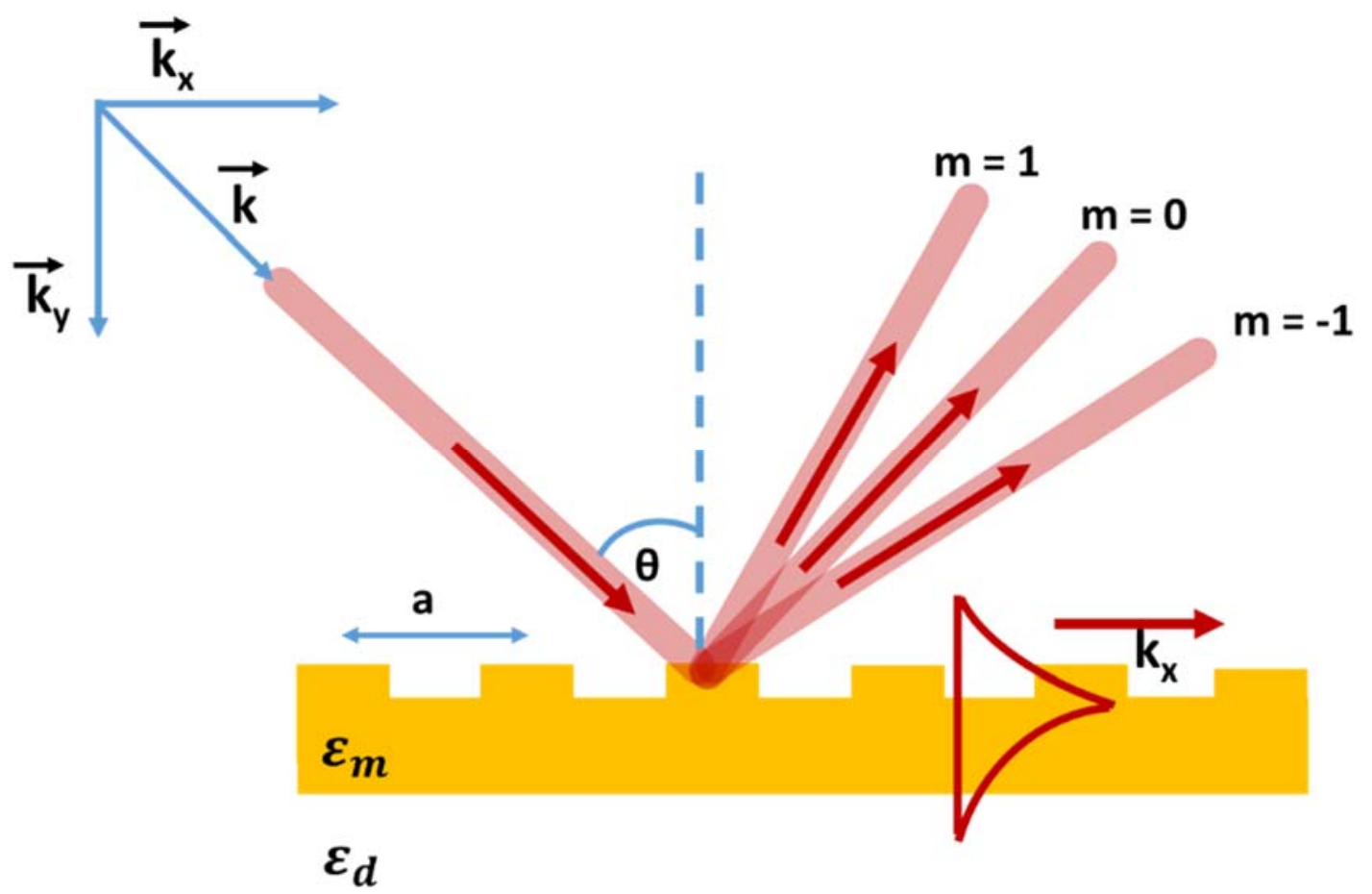

Figure 1-2: The configuration of the SPR grating coupling.

The wave-vector component of the diffracted light that is parallel to the surface is given by [50]:

$K_{x}=\frac{\omega}{c} \sin (\theta) \pm n \frac{2 \pi}{a} \quad n=0,1,2,3 \ldots$ Equation 1-4

Therefore, the grating coupler can increase the parallel wave vector component by an amount related to the grating period. The diffracted light can couple to a surface plasmon 
and then excite the surface plasmon when the momentum of diffracted light parallel to the grating surface is equal to the propagation constant of the surface plasmon.

However, the resonance coupling appears as a dip in the reflectivity of the light spectrum, as seen in Figure 1-3, which is traditionally tracked by measuring the wavelength, the incident angle, or the intensity of the reflected light $[51,52]$.

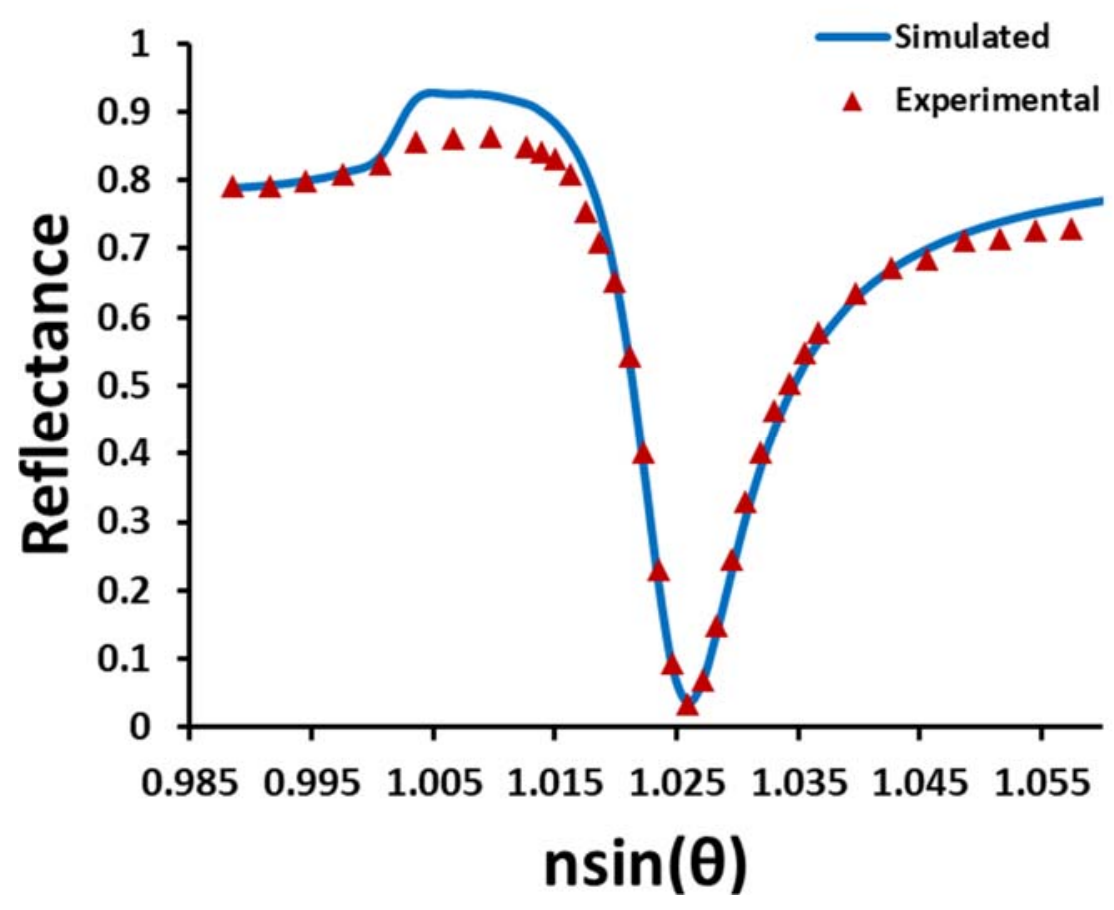

Figure 1-3: Reflectivity profile against the incident angle of a monochromatic p-polarized light.

The angular sensitivity of the SPR depends on the penetration length of the evanescent field created by SPR which it is depending on the dielectric constant, the thickness of the metal film, and the wavelength used to excite the SPR wave [53]. As described earlier, the existence of this surface plasmon wave is dictated by the electromagnetic properties of a metal and a dielectric (sample medium) interface. Therefore, the dip in the reflectivity is extremely sensitive to the change in the dielectric constant near the metal/dielectric 
interface. Thus, and due to the high sensitivity of SPR techniques, it is used to study and detect molecular assemblies by measuring the optical parameters associated with the change in the resonant condition.

\subsection{SPR Optical Biosensors}

Optical biosensors based on SPR has been demonstrated in the early of 1980s [54]. Over the past decade, the SPR sensing technology has been performed, and numerous SPR sensor platforms and data processing and analysis methods have been developed. SPRbased sensors were used in a widespread application in fundamental biological studies [55, 56] health science research [57], drug discovery [56], clinical diagnosis [58], and environmental monitoring [59-61]. Many research groups have studied the SPR biosensors and their applications for many years and thousands of research papers have been published. The performance characteristics of SPR biosensors include sensitivity, linearity, resolution, and limit of detection have been studied and improved [62-73]. SPR allows for the qualitative and quantitative measurements of biomolecular interactions in real-time without requiring a labelling procedure [74-79]. Today, the development of SPR is geared towards the design of compact, low-cost, and sensitive biosensors $[69,80]$.

\subsection{Electrochemical-Surface Plasmon Resonance Technique (EC-SPR)}

Despite the outstanding sensitivity demonstrated in well-controlled lab conditions, the conventional SPR configuration has not reached widespread deployment in field applications as it demands high environmental control and accurate mechanical precision of the active opto-mechanical components. Even though the conventional SPR technique is very sensitive to the refractive index changes at the surface, it still lacks sensitivity to probe redox species with weakly absorbing chromophores and/or at low surface 
concentrations. A limiting factor for performance has been the subtle refractive index changes of a redox adsorbate under interrogation in cases of a miniscule amount of mass deposited onto a sensor surface. Moreover, the signal fluctuations caused by temperature fluctuations and mechanical vibrations and the non-specific binding on the surface can cause false changes in the refractive index, leading to misinterpreted signals. An alternative to the SPR technique that has been proposed recently is the electrochemical surface plasmon resonance (EC-SPR) technology [81-84], which keeps the benefits and mitigates the weakness of the original SPR technique. The effects of an electrical potential on the properties of surface plasmon have been early demonstrated for both gold and silver electrodes in contact with an electrolyte solution [85]. The mechanisms behind this effect was identified as variations in the electron density at the metal surface, ion adsorption and changes in the optical properties of double layer [86]. In 2000, Tao group presented and demonstrated the basic concepts of the SPR angular shift in response to the potential modulation [87]. The development of a basic and quantitative formalism and experimental validation by the group was laid a solid theoretical foundation for research and development of electrochemical surface plasmon resonance sensing [82]. Next, the group was focused mainly on electrochemical SPR imaging detection to enhance the performance and practicality of the new technique [88]. Using the new technique in imaging mode, mass sensitivity was improved for detecting small molecules ( $<100 \mathrm{Da})$ [89-91] and determining kinetic parameters of biomolecular binding events at the device active interface [83]. Moreover, the technique shows the ability to study local electrochemical phenomena, such as the electrochemical activities of nanomaterials which are critical for creating novel catalysts, for developing ultrasensitive sensors, and for understanding fundamental nano- 
electrochemistry [81-83]. A biosensor based on the EC-SPR technique has been developed by the Meunier group [92] by immobilizing onto the SPR surface a thiolated-DNA strand that has been conjugated to a redox methylene blue (MB) probe. The MB probe displays a strong and robust change in its optical absorption associated with its redox transition. The DNA strand features a stem-loop that initially constrains the MB label to stay close to the electrode surface (the ON configuration). In the absence of the target DNA, an applied potential modulation at the electrode interface that oscillates around the formal potential of the redox probe will modulate the optical SPR signal, which can then be used to provide information on the redox activity of the MB probe. As a result, a strong amplitude in the optical signal reports the absence of the target analyte. When the target DNA is present in the sample under scrutiny, it will then bind to the immobilized DNA strand and such an event will open the original stem loop displacing the MB farther away from the electrode surface (the OFF configuration). This configuration will then reduce the number and probability of $\mathrm{MB}$ molecules that undergo a redox transition under an applied potential modulation, and therefore an optical signal of lower amplitude is detected indicating the presence of the target analyte. Although the overall concept was successfully demonstrated, under this sensing strategy the OFF configuration (when the redox probe has been displaced further away from the electric interface yet resides close to the surface) still provides a small but non-negligible chance for the electron transfer process to occur, which can generate an undesirable optical signal. Therefore, for this embodiment, the optical background signal (under a high concentration of the target DNA) was relatively high with a negative impact in the detection sensitivity, which was reported in the nanomolar range. 
In this work, an electrochemically-modulated surface plasmon resonance (EC-SPR) technique was developed for challenging investigations in different redox assemblies. The principle of the technique is based on the nano-scale confined and sensitive interplay between a surface plasmon wave and a redox-active probe featuring an optical transition when undergoing a faradaic redox process. Such faradaic process is electrochemically controlled by modulating the surface electric potential of the SPR platform to create an optical output signal that is substantially immune to the negative impacts of background environment and enabling the identification and quantification minute properties linked to a molecular event of interest. Such a methodology is highly selective as the monitored analytical signal is both optically and electrochemically locked to the spectroelectrochemical fingerprints of either naturally existing or specifically designed biomarkers for detection. We expect with such improvements, the EC-SPR device brings substantial improvements for I) developing high sensitive detection of multiplexed targets and overcoming the effects of background, II) opens new opportunities to investigate a variety of redox-transduced events with a small difference in their molar and with extremely low surface densities.

\subsection{Outline of the Dissertation}

In chapter 2, the design, characterization, and optimization of an EC-SPR device are described. A well-characterized, redox-active probe, cytochrome c protein are used as a model system. Experimental measurements and theoretical calculations are performed to define the optimal parameters for successfully adapted EC-SPR device for spectroelectrochemical measurements. In chapter 3, a combination between the EC-SPR device and an optical impedance spectroscopy technique (OIS) to study the redox reaction 
kinetics of a surface-adsorbed redox species is applied and demonstrated. The high sensitivity and ability of the EC-SPR device to implementing at a broad potential range and at high modulation frequencies was the motivation to this combination. The impact of different multilayer assembly on the electron-transfer rate of bound cytochrome c protein is examined. In chapters 4 and 5, a novel immunosensor- based strategy for direct detection of biological targets by incorporating a sandwich bioassay on the electrochemically modulated SPR platform is developed and demonstrated. The EC-SPR device shows the capability to directly detect and reproducibly identify the presence of a hemagglutinin (HA) protein from the H5N1 avian influenza A virus and gram-negative bacteria in PBS buffer solution. The experimental results with viral and endotoxin biomaterials have produced outstanding limits of detection in the pico-molar range. In chapter 6, concluding considerations are presented and an outlook is offered for possible applications of EC-SPR device. 


\section{CHAPTER 2}

\section{ELECTROCHEMICAL SURFACE PLASMON RESONANCE (EC- SPR): DESIGN AND CHARACTERIZATION}

The precise design of the EC-SPR technology is emerging as a key factor in the performance of implementing the device in electrochemical modulation measurements. Several major attributes of an EC-SPR device are available for optimization to get superior performance and successfully adapted for spectroelectrochemical measurements. Defining a proper SPR platform and its properties are very crucial for obtaining a strong resonant condition (a sharp inflection in the reflectance curve that almost vanishes at the resonant condition) and for achieving surface stability under applied potentials. Moreover, the selection of an appropriate incident angle and a well-chosen specific wavelength are critical for optimizing the difference in the modulated optical signal which will have a major the EC-SPR sensitivity for a broad range of analytical applications. In this chapter, a rational methodology for the design, characterization, and optimization of an EC-SPR device, based on SPR platform in an aqueous solution is described.

\subsection{Conventional Surface Plasmon Resonance (SPR)}

The development of an ultrasensitive sensors based in the conventional SPR technique strongly depends on getting a minimum reflectance and a narrow angular width (full width at half maximum, FWHM). This may be achieved through careful design and 
fabrication procedures of the SPR platform. Gold and silver are usually used as a platform of SPR devices. In the following section, the construction of a proper SPR platform for highly sensitive biosensing technology is designed. In addition, the cleaning procedure of the glass slides, the film deposition process, and the characterization of gold and silver thin films are described.

\subsubsection{Surface Plasmon Resonance Platform Preparation}

Microscope glass slides were used as substrates. The following are the cleaning procedure used for preparing the microscope glass slides for the thin film coating process. An ultrasonic cleaner system (Branson, 2510, Hampton, NH 03842) with $60{ }^{\circ} \mathrm{C}$ heating was used to clean the soda-lime glass slides (VWR, 48300-048). The glass slides were placed in a glass separator rack and put inside the glass tub. The separator rack was immersed in the solution of DI water and triton mixture $0.1 \%$ DI $(1 \mathrm{~mL}$ Triton and 1000 $\mathrm{mL}$ DI water) until all the substrates were completely covered. Then, the slides were sonicated for 10 minutes, meanwhile, cotton swabs were used to vigorously scrub each slide. Next, the glass separator rack was rinsed with DI water and sonicated in a DI water solution for 10 minutes. The organic contaminations on the surface was removed by immersing the glass slides in a fresh piranha solution (1:3 of hydrogen peroxide to sulfuric acid) and sonicated for 10 minutes. After rinsing the glass rack with DI water, the glass slides were placed in a tub of acetone solution and then in methanol solution and sonicated respectively, for 10 minutes each. Finally, the glass slides were removed from the methanol solution, dried with nitrogen gas $\left(N_{2}\right)$ and then placed in an oven for 1 hour at $110^{\circ} \mathrm{C}$. After this step, the glass slides were ready for the thin film deposition process. 


\subsubsection{Metal Thin Film Deposition}

A dc magnetron sputtering technique (Nano-Master) was used for the gold and silver thin film deposition on the surface of the cleaned microscope glass slides at room temperature. The cleaned microscope glass slides were placed on the sample holder. The load lock chamber (LLC) was vented. The door of the load lock chamber (LLC) was open automatically when the pressure inside the chamber becomes around $6 \times 10^{-2}$ Torr. The sample holder was loaded into the LLC and then the LLC doors are carefully closed. The

pressure inside the LLC was pumped down. After the pressure reaches at least $2 \times 10^{-2}$ Torr, the sample holder was sent to chamber. The rate of a metal thin film deposition using a sputtering machine is proportional to the sputtering power, the pressure of the Argon gas (Ar), and the time of deposition. Three different deposition times at fixed sputtering power (100 Watt) and fixed Ar gas $(150 \mathrm{sccm})$ were used for measuring the deposition rate, as will be described in the next section.

\subsubsection{Calibrated Deposition Rate}

The de deposition rate for gold and silver thin film coating at fixed flow rate of Ar gas and at room temperature was determined. For gold thin film deposition, three different deposition times 60 seconds, 70 seconds, and 90 seconds were used. A conventional SPR technique was used to determine the thickness of the three gold thin film samples. The setup shown in Figure 2-1 was employed for the angular interrogation experiments. 


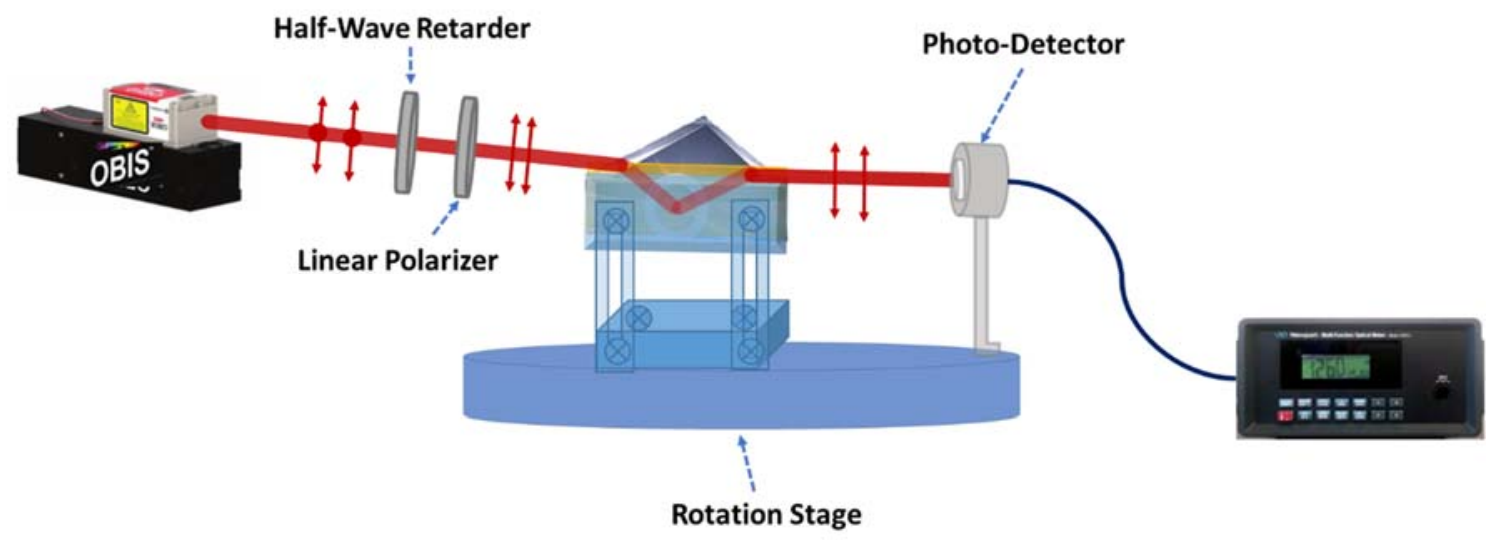

Figure 2-1: Diagram of a conventional surface plasmon resonance (SPR). CW laser with combination of a half-wave plate and a linear polarizer to establish linearly polarized light at transverse-magnetic (TM) polarization was used as an excitation light. Prism/metalcoated substrate configuration was set up on a rotation stage to precisely control the incident angle. An index matching gel was used to optically couple the metal-coated substrate to an equilateral BK7 glass prism. A photo-detector was used to collect the reflected light.

A CW laser (Obis from Coherent) was deployed in combination with a half-wave plate and a linear polarizer to establish linearly polarized light at transverse-magnetic (TM) polarization. An equilateral $\mathrm{BK} 7$ glass prism $\left(60^{\circ}\right.$ equilateral prism, Edmund Optics) was optically coupled to the metal-coated substrate using index matching gel $(\mathrm{nD}=1.52$, Cargille) and then was mounted on a rotation stage (Quadra Chek 100 rotation stage) to precisely control the incident angle. The laser beam was directed towards the entrance facet of the coupling prism to excite the surface plasmon wave. By rotating the stage, the incident angle was scanned and the reflected optical intensity at different angles of incidence was monitored continuously by a power meter (Newport, model 1930C). The SPR reflectance curves of the three samples using $660 \mathrm{~nm}$ excitation wavelength were measured and plotted 
in Figure 2-2. A Mathematica program based on the transfer-matrix method was used to calculate the thickness and the optical constants of a thin film (Appendix A-1) at $660 \mathrm{~nm}$ excitation wavelength. Three-layer (BK7/gold/air) model was used for the calculated result. The refractive index of air is approximately 1.00 , and the refractive index of the soda-lime glass of the microscope slides was assumed to be similar to the BK7 glass. The refractive index of the $\mathrm{BK} 7$ is given by the following equation:

$n_{B K 7}=\sqrt{\left(\frac{1.03961212 \lambda^{2}}{\lambda^{2}-0.00600069867}+\frac{0.231792344 \lambda^{2}}{\lambda^{2}-0.0200179144}+\frac{1.03961212 \lambda^{2}}{\lambda^{2}-103.560653}\right)}$

Equation 2-1

Where $\lambda$ is the wavelength (in $\mu \mathrm{m}$ for the numerical described included) of the incident light. The extinction coefficients of air and BK7 are assumed to be zero.

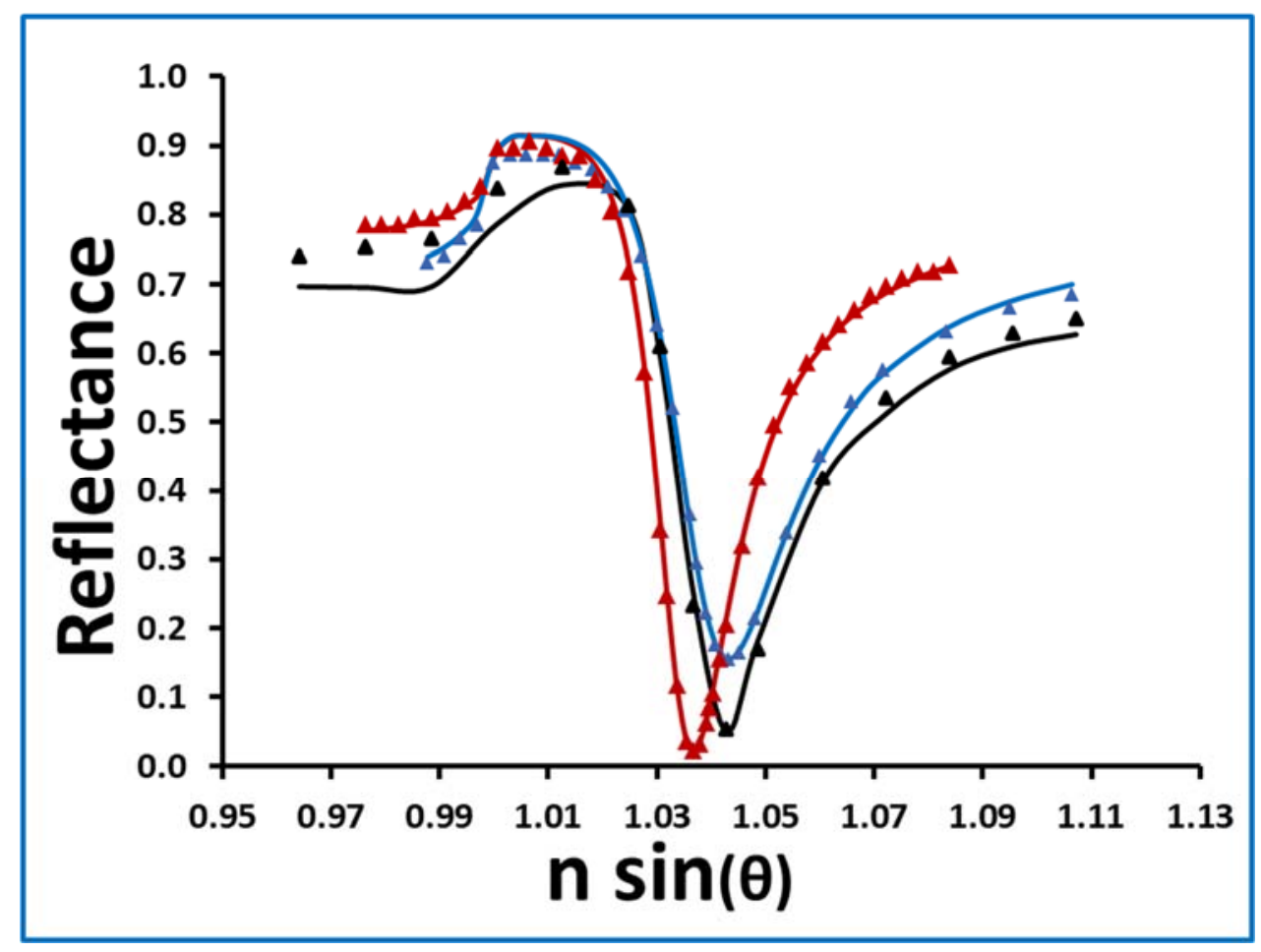

Figure 2-2: Measured and calculated SPR reflectance profile to determine the thickness of the gold thin films at three deposition times. The red, blue, and black dots are measured data obtained by the conventional SPR setup at 60 seconds, 70 seconds, and 90 seconds, 
respectively. The red, blue, and black solid lines are the calculated SPR reflectivity curves with the optical constants and the thickness after optimization for 60 seconds, 70 seconds, and 90 seconds, respectively.

Figure 2-2 shows the measured SPR reflectance curves obtained by the conventional SPR setup, and the calculated SPR reflectance curves based on a three-layer (BK7/silver/air) model at $660 \mathrm{~nm}$ excitation wavelength for three samples. The thickness of the gold thin film was determined by minimizing the difference between the calculated results and the measured data obtained by the conventional SPR setup. A well-match was observed between the calculated results and the experimental data. The thicknesses of the gold thin film measured at the three deposition times ( 60 seconds, 70 seconds, and 90 seconds) were $32 \mathrm{~nm}, 35 \mathrm{~nm}$, and $45 \mathrm{~nm}$, respectively. Finally, a plot of the measured gold thickness against the deposition times were used to determine the calibrated rate, Figure 2-3.

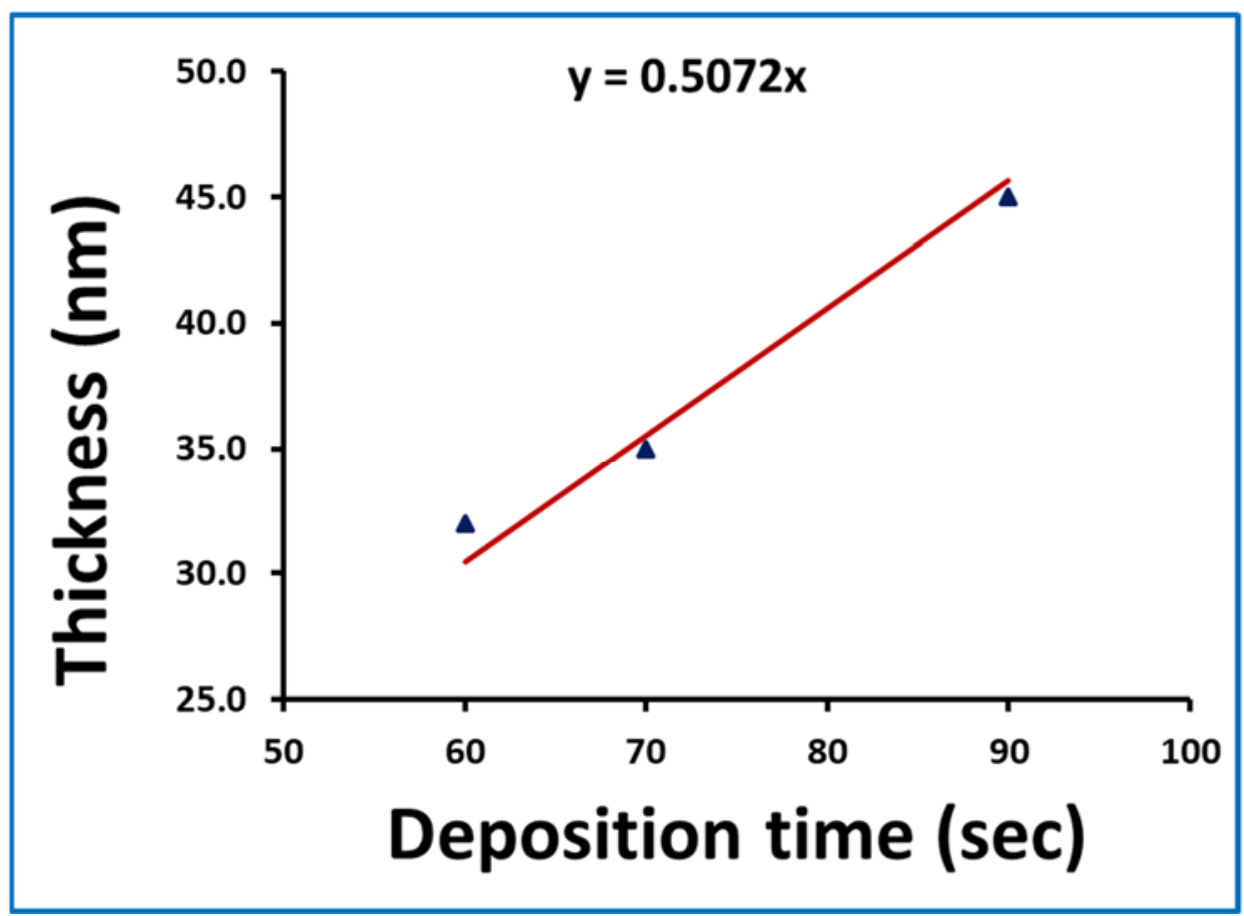


Figure 2-3: The measured gold thickness against the deposition time. The deposition rate is represented by the slope of the linear fit.

The calibrated deposition rate to form a thin film of gold using the dc magnetron sputtering technique was measured by a linear fitting to be $0.5 \mathrm{~nm} / \mathrm{second}$. The same procedure was carried out to determine the calibrated deposition rate to form a silver thin film on cleaned microscope glass slides at room temperature. The data are shown in Appendix A-2. The calibrated deposition rate to form a thin film of silver was measured to be $1 \mathrm{~nm} / \mathrm{second}$.

\subsubsection{SPR Platform Characterization}

The conventional SPR configuration based on the angular interrogation experiments was used for gold and silver thin films characterization. The same setup shown in Figure 2-2 was employed. Five wavelengths: $552 \mathrm{~nm}, 637 \mathrm{~nm}, 660 \mathrm{~nm}, 685 \mathrm{~nm}$, and $785 \mathrm{~nm}$ were used to excite the surface plasmon wave. Calculated results based on a Mathematica program for a multilayer model (Appendix A-1) was used to match the measured SPR reflectance curves. The optical constants of the gold and silver thin films at each wavelength were obtained by minimizing the difference between the measured and the calculated data.

\subsubsection{Gold Thin Film Characterization}

The optical constants of a gold thin film were examined at several wavelengths. The SPR reflectance curves collected in air at the five excitation wavelengths are shown in Figure 2-4, which also includes the corresponding calculated results obtained from the retrieved optical constants. For this calculation, a three-layer (BK7/gold /air) model was used. 


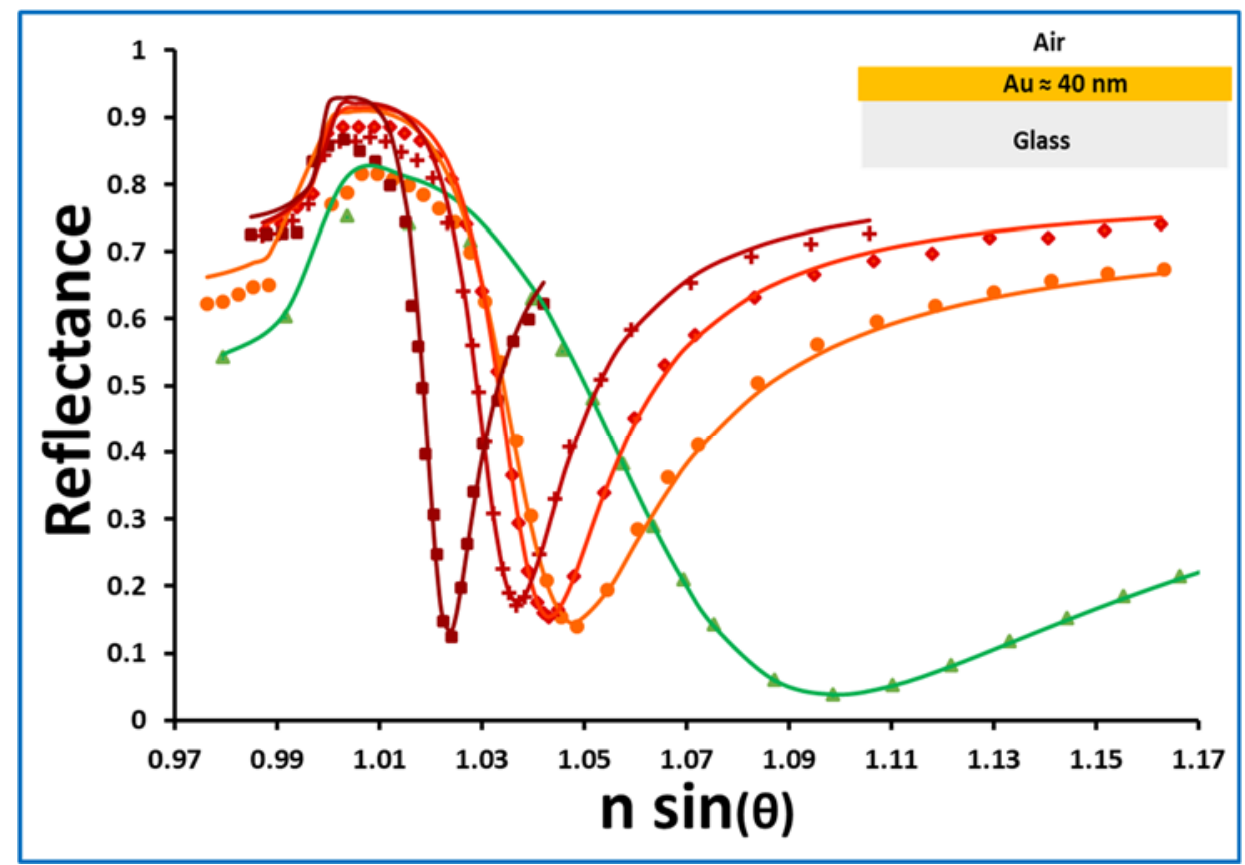

Figure 2-4: The optical constants examination of the gold thin film in air. The dotted curves are measured data obtained by the conventional SPR setup at each excitation wavelength. The solid lines are calculated SPR reflectance curves based on a Mathematica program and the retrieved optical constants ( $n$ and $k$ ) and film thickness $(t)$. The wavelengths of light are $552 \mathrm{~nm}, 637 \mathrm{~nm}, 660 \mathrm{~nm}, 685 \mathrm{~nm}$, and $785 \mathrm{~nm}$.

The numerical analysis deploys a large number of data points (reflectance values at several angles of incidence) to retrieve three parameters $(n, k$, and $t)$. The retrieved optical constants $(n$ and $k$ ) of the gold film at each excitation wavelength are listed in Table 2-1.

\subsubsection{Silver Thin Film Characterization}

A bilayered stack of silver and gold was then used to determine the optical constants of the silver thin film at the same five excitation wavelengths. The same setup shown in Figure 2-2 was used to determine the SPR reflectance curves for the bilayered (silver/gold) platform on a PBS buffer solution at each excitation wavelength. The purpose of collecting 
these measurements in the PBS buffer solution was to test the stability of the bilayered (silver/gold) platform in an aqueous environment. For the calculated data, a four-layer (BK7/silver/gold/PBS buffer solution) model was used. The thickness of the gold film was aimed and fixed at $5 \mathrm{~nm}$. The optical constants of the gold thin film determined in the previous section (2.1.4.1) were used. The measured and calculated SPR reflectance curves using the five excitation wavelengths are shown in Figure 2-5.

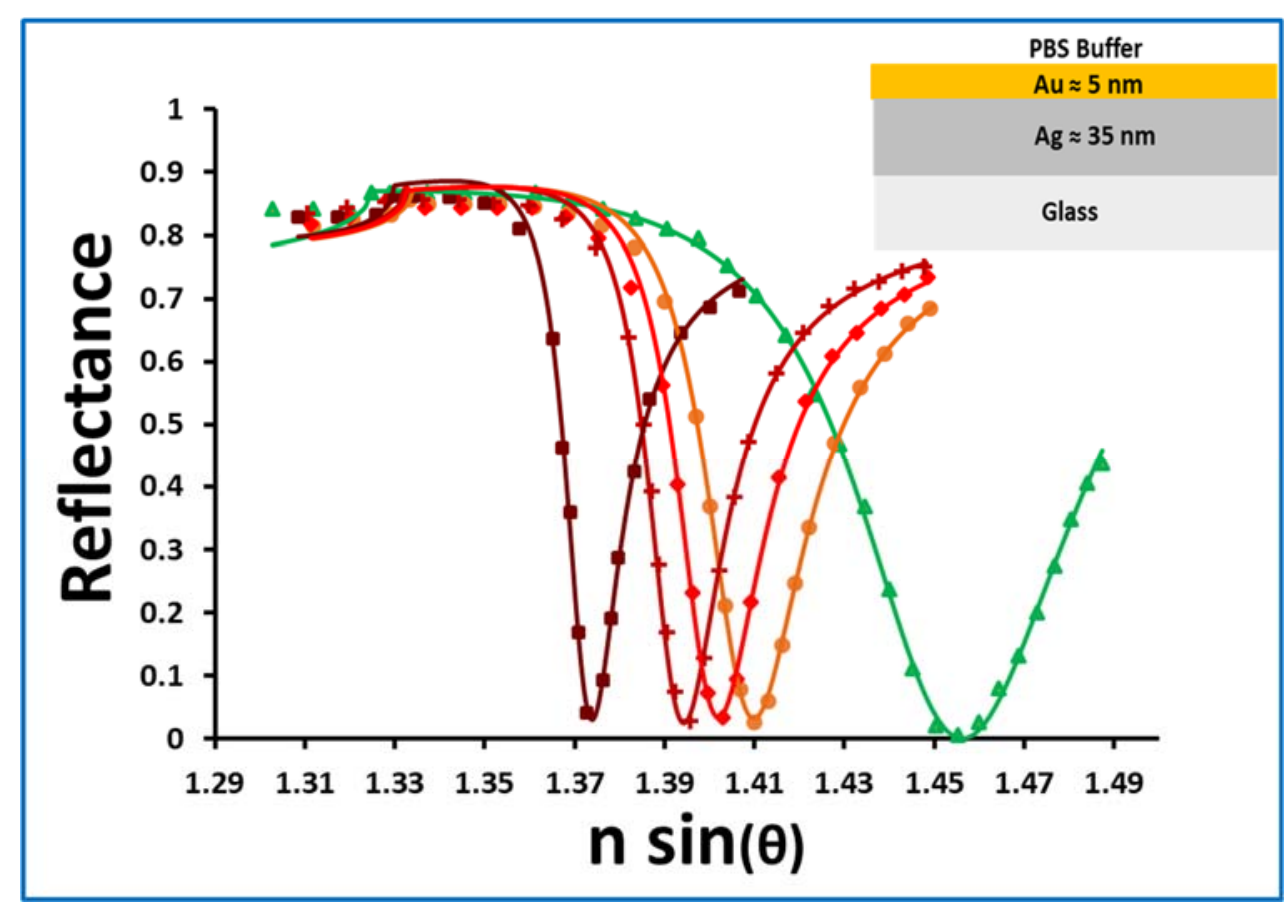

Figure 2-5: The optical constants examination of the silver thin film in PBS buffer solution. The dotted curves are experimental results obtained by the conventional SPR setup at each excitation wavelength. The solid lines are the calculated SPR reflectance curves using the determined optical constants and thicknesses for the two metal layers. The excitation wavelengths are $52 \mathrm{~nm}, 637 \mathrm{~nm}, 660 \mathrm{~nm}, 685 \mathrm{~nm}$, and $785 \mathrm{~nm}$.

The fitting parameters that provide the optical constants of the silver thin film $(35 \mathrm{~nm})$ at each excitation wavelength are listed in Table 2-1. 
Table 2-1: The optical constant of gold and silver thin films were extracted by

minimizing the difference between the measured and calculated SPR reflectance curves.

\begin{tabular}{|c|c|c|c|c|}
\hline \multirow{2}{*}{ Wavelength (nm) } & \multicolumn{2}{|c|}{ Silver thin film } & \multicolumn{2}{c|}{ Gold thin film } \\
\hline & $\mathrm{n}$ & $\mathrm{k}$ & $\mathrm{n}$ & $\mathrm{k}$ \\
\hline 552 & 0.0901 & 3.3320 & 0.3856 & 2.6144 \\
\hline 637 & 0.1307 & 4.3535 & 0.2082 & 3.7340 \\
\hline 660 & 0.1520 & 4.6014 & 0.1596 & 3.8281 \\
\hline 685 & 0.1611 & 4.7767 & 0.1553 & 4.1589 \\
\hline 785 & 0.1762 & 5.5224 & 0.1937 & 5.2840 \\
\hline
\end{tabular}

The data of the optical constants of the gold and silver thin films shown in Table 2-1 are in agreement with the works of literature at the same wavelengths and thickness [93-95]. These data were used to design and enhance the performance of implementing the ECSPR device in electrochemical modulation measurements.

\subsection{Surface Plasmon Resonans Platforms Thicknesses Optimization}

Three configurations of the SPR platform \{gold, silver, and bilayered (gold/silver)\} were proposed and theoretically tested in an aqueous environment. For each configuration, the thicknesses of the layers were optimized. A typical reflectivity for an SPR under angular interrogation for five different thicknesses $(30 \mathrm{~nm}, 35 \mathrm{~nm}, 40 \mathrm{~nm}, 45 \mathrm{~nm}$, and 50 $\mathrm{nm})$ and using five excitation wavelengths $(552 \mathrm{~nm}, 637 \mathrm{~nm}, 660 \mathrm{~nm}, 685 \mathrm{~nm}$, and 785 $\mathrm{nm}$ ) were calculated based on the previously determined optical constants for gold and silver. A Mathematica program based on a transfer-matrix technique (Appendix A-3) was used to calculate the SPR reflectance profile for a multilayer model at each wavelength. Figure 2-6 shows the calculated SPR reflectance curves against the effective refractive 
index inside the prism, $n \sin \theta$, for the gold, silver, and bilayered (gold/silver) platforms at the $552 \mathrm{~nm}$ excitation wavelength.

(a)

(b)
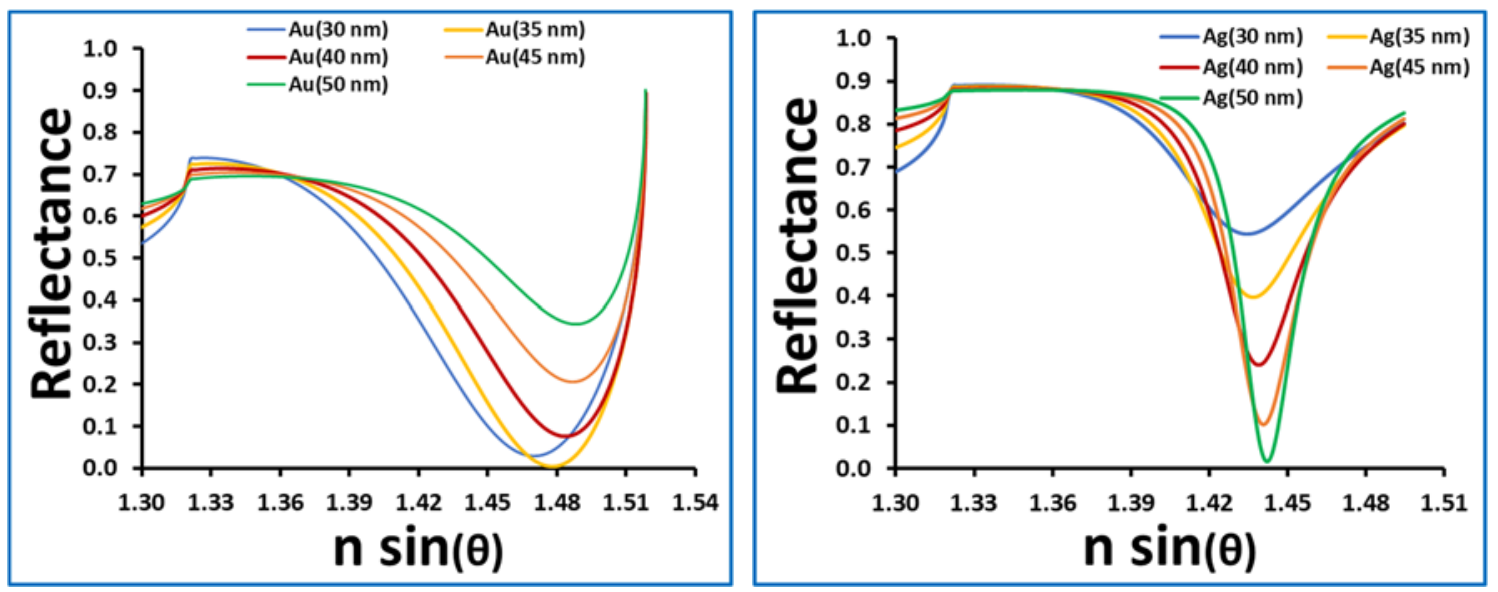

(c)

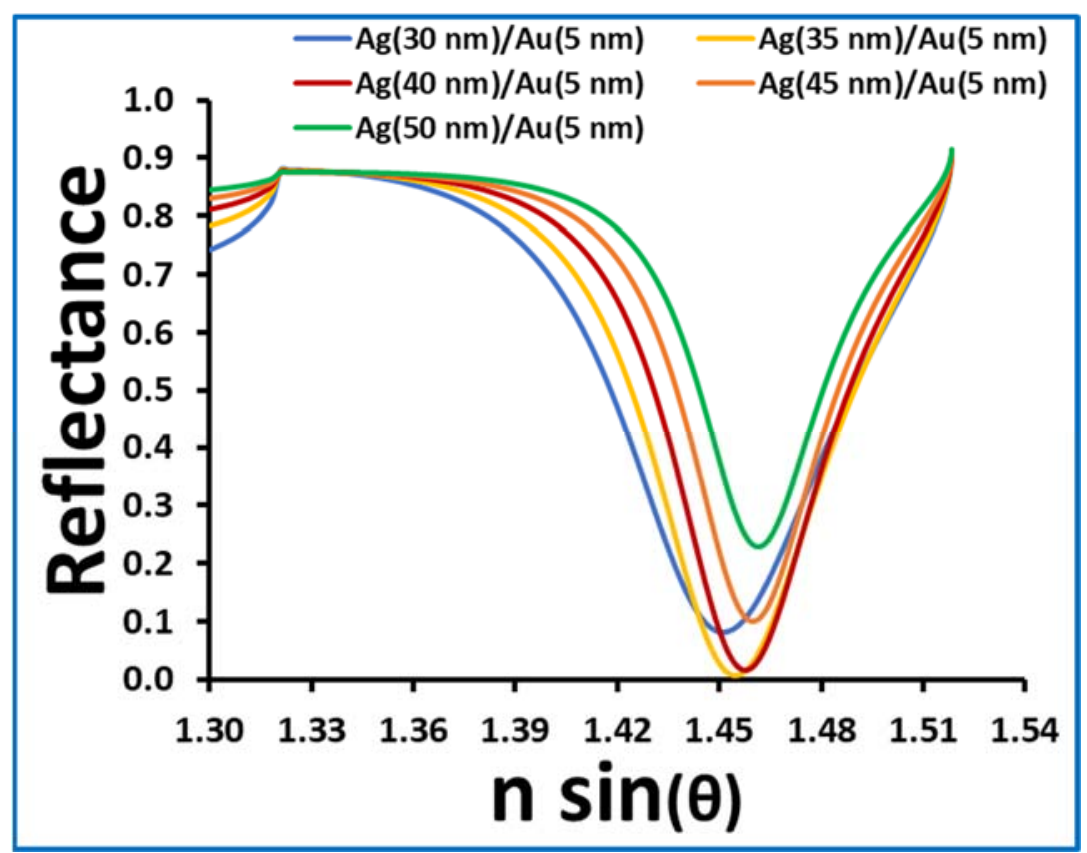


Figure 2-6: The calculated SPR reflectance curves for (a) gold thin film, (b) silver thin film with thickness of $30 \mathrm{~nm}, 35 \mathrm{~nm}, 40 \mathrm{~nm}, 45 \mathrm{~nm}$, and $50 \mathrm{~nm}$, and (c) bilayered (silver/gold) thin film with silver thin film thickness of $30 \mathrm{~nm}, 35 \mathrm{~nm}, 40 \mathrm{~nm}, 45 \mathrm{~nm}$, and $50 \mathrm{~nm}$ and a fixed thickness of gold thin film $(5 \mathrm{~nm})$. The following values are used for the calculations: $n_{\text {prism }}=1.51842, n_{\text {gold }}=0.38559+2.61439 i, n_{\text {silver }}=0.0719+3.2431$, and $n_{\text {Buffer }}=$ 1.32045. The excitation wavelength is $552 \mathrm{~nm}$.

The calculated SPR reflectance curves for the three configurations using the other excitation wavelength $(637 \mathrm{~nm}, 660 \mathrm{~nm}, 685 \mathrm{~nm}$, and $785 \mathrm{~nm})$ are shown in Appendix A4. Based on the calculated results, the optimal thicknesses of the three configurations that provide the best performance of the SPR technique in an aqueous environment are listed in Table 2-2.

Table 2-2: The optimal thickness of the three configurations (gold, silver, and bilayered [silver/gold]) platform that demonstrated a strong resonant condition in an aqueous environment.

\begin{tabular}{|c|c|c|c|}
\hline Configuration & Silver thin film & Gold thin film & Bilayered (silver/gold) \\
\hline Wavlength (nm) & $\begin{array}{l}\text { Optimal thickness } \\
(\mathrm{nm})\end{array}$ & $\begin{array}{l}\text { Optimal thickness } \\
\text { (nm) }\end{array}$ & $\begin{array}{l}\text { Optimal thickness } \\
(\mathrm{nm})\end{array}$ \\
\hline 552 & 50 & 35 & $35 / 5$ \\
\hline 637 & 45 & 45 & $40 / 5$ \\
\hline 660 & 45 & 50 & $40 / 5$ \\
\hline 685 & 45 & 50 & $40 / 5$ \\
\hline 785 & 45 & 45 & $40 / 5$ \\
\hline
\end{tabular}

Next, using the optimal thickness and the five excitation wavelengths, the calculated SPR reflectance curves for the three configurations \{gold, silver and bilayered (silver/gold) platform $\}$ were plotted in one graph. Figure 2-7 shows the calculated results using $552 \mathrm{~nm}$ 
as the light source for the calculated. The calculated results using $637 \mathrm{~nm}, 660 \mathrm{~nm}, 685 \mathrm{~nm}$, and $785 \mathrm{~nm}$ as the light sources for the calculated were shown in Appendix A-5.

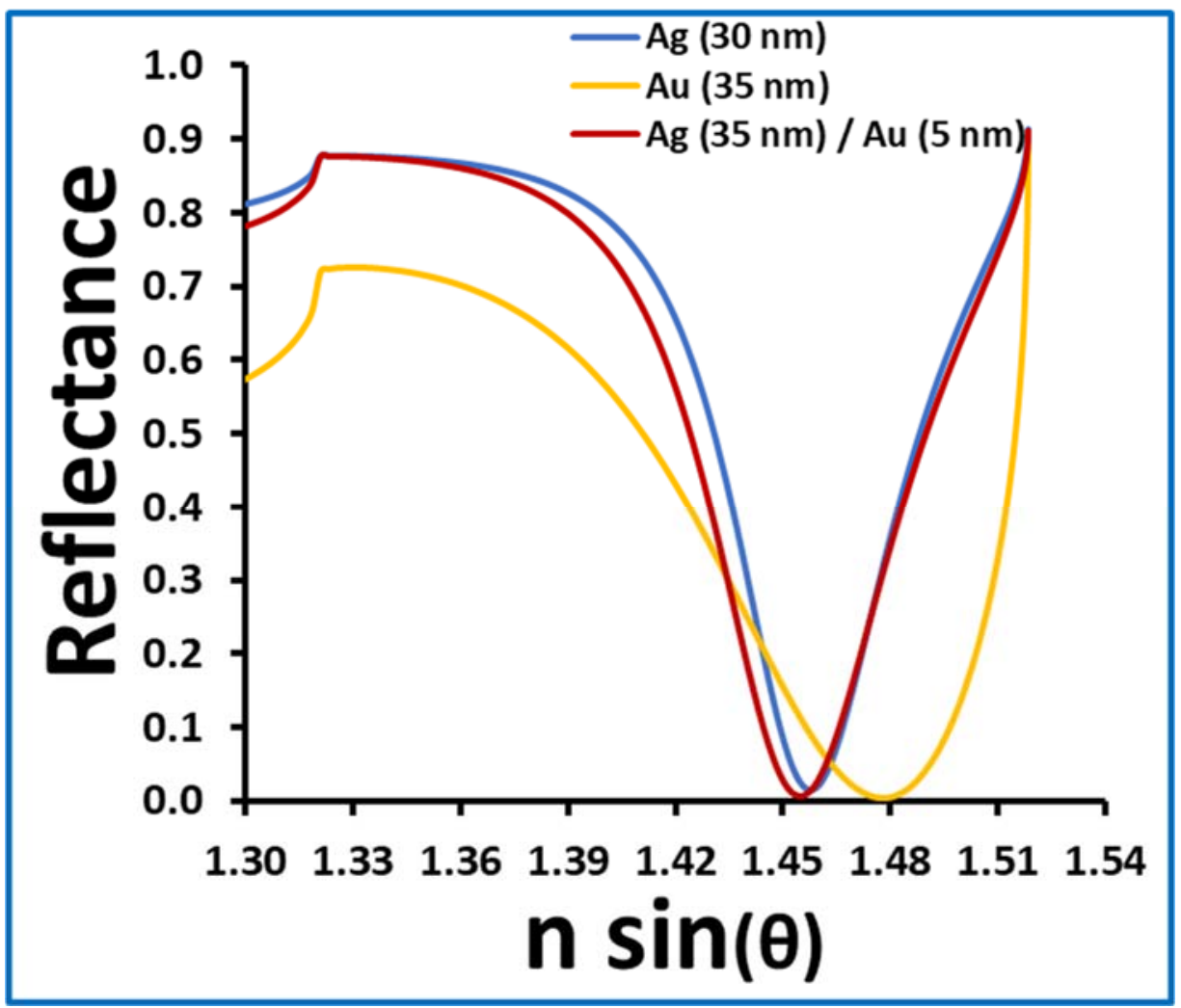

Figure 2-7: The calculated SPR reflectance curves for silver $(30 \mathrm{~nm})$, gold $(35 \mathrm{~nm})$ and bilayered silver $(35 \mathrm{~nm}) /$ gold $(5 \mathrm{~nm})$ thin films. The following values were used for the calculations: $\mathrm{n}_{\text {prism }}=1.51842, \mathrm{n}_{\text {gold }}=0.38559+2.61439 \mathrm{i}, \mathrm{n}_{\text {silver }}=0.0719+3.2431 \mathrm{i}, \mathrm{n}_{\text {Buffer }}$ $=1.32045$, and the wavelength of light is $552 \mathrm{~nm}$.

The results demonstrated that the silver configuration yields a sharper and narrower SPR dip profile than the other two configurations \{gold and bilayered (silver/gold)\} at all excitation wavelengths investigated. However, the silver thin film has poor stability under potential modulation and low chemical stability in different solution environments, in contrast to a gold thin film, which has high chemical stability and resistance to oxidation in many environments [96]. For these reasons, a bilayered (gold/silver) platform was used 
in the EC-SPR device. Therefore, the silver thin film improves the SPR sensitivity, whereas the gold thin film keeps the chemical stability of the SPR platform for adapting the ECSPR device for spectroelectrochemical measurements [97].

\subsection{Electrochemical-Surface Plasmon Resonance Device (EC-SPR)}

A well-controlled fabrication process of the SPR platform was then carried out to secure the benefits of the design efforts previously described and deliver a SPR structure with a strong optical resonance of high performance. As already mentioned, the EC-SPR device was fabricated based on the bilayered (silver/gold) configuration as this configuration provides a narrow SPR dip profile and high chemical stability. A cleaned microscope glass slide was coated with a silver thin film of a $35 \mathrm{~nm}$ thickness and then followed by a $5 \mathrm{~nm}$ gold thin film for protection. Next, the EC-SPR device was constructed and tested. The SPR reflectance measurements and the cyclic voltammetry (CV) measurements were used to characterize the EC-SPR response for optical measurements at low surface densities. A well-characterized, redox-active probe, the cytochrome c protein was used as a model system.

\subsubsection{Micro-Electrochemical Flow Cell Fabrication}

A homemade micro-electrochemical flow cell with a three-electrode configuration was fabricated to handle the liquid injection. Figure 2-8 (a) shows the scheme of the flow cell. 
(a)

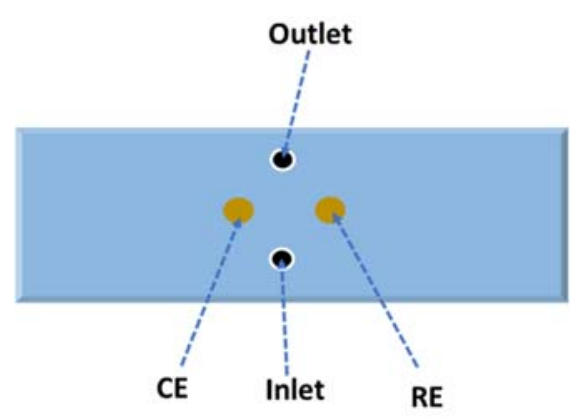

(b)

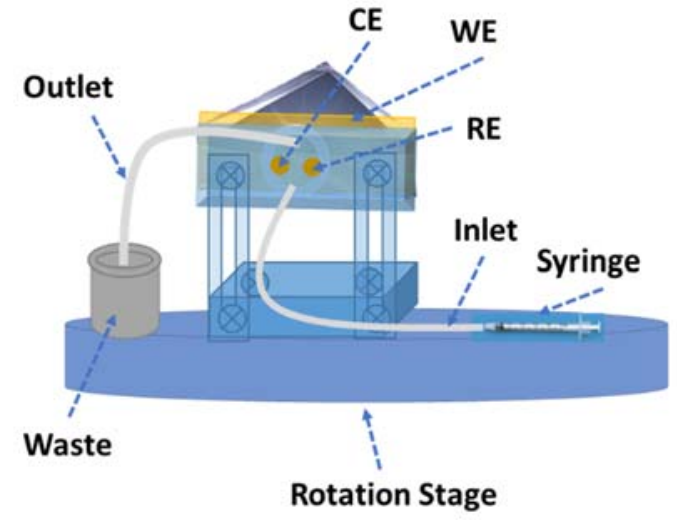

Figure 2-8: (a) Cross-section of the electrochemical flow cell with liquid inlet and outlet, and two gold electrodes. (b) Schematic of the electrochemical flow cell with threeelectrode attached to the prism coupling unit in the Kretschmann configuration and mounted on a rotating stage. A syringe pump was used for handling the liquid injection into the electrochemical flow cell and a plastic tube for the liquid waste.

The body of the flow cell was made of silica and has four holes. The counter and the reference electrodes were inserted into two holes, and the other two were used for injection and rinsing the liquid samples. Two gold-plated pins (surface area $=2.85 \mathrm{~mm}^{2}$ ) (MOUSER ELECTRONICS ${ }^{\circledR}$, Mansfield, TX, USA) were used as pseudo-reference (RE) and counter electrodes (CE). A bilayered (silver/gold) platform was served as a working electrode (WE). An equilateral BK7 glass prism (60 equilateral prism, Edmund Optics) was optically coupled to the bilayered (silver/gold) platform using index matching gel $\left(\mathrm{n}_{\mathrm{D}}=\right.$ 1.52, Cargille) and then was mounted on a rotation stage (Quadra Chek 100 rotation stage), Figure 2-8 (b). A manually activated syringe pump was used to inject all solutions into the electrochemical flow cell. The volume of the electrochemical flow cell was designed by a rubber gasket placed between the body and the platform to be approximately $50 \mu 1$. 


\subsubsection{EC-SPR Setup}

The EC-SPR setup has two parts: A homemade micro-electrochemical flow cell with a three-electrode configuration as described in Section 2.3.1 and the optical part which is a conventional SPR setup that is based on the widely used Krectchmann configuration. The schematic of the EC-SPR setup is illustrated in Figure 2-9

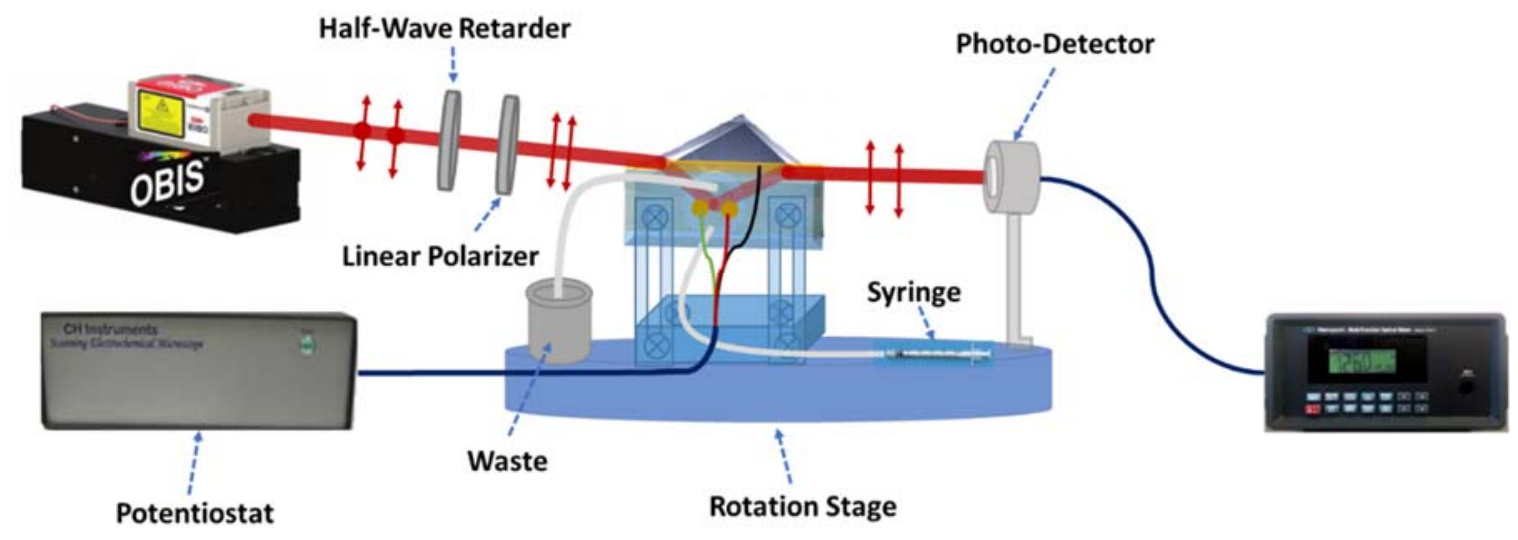

Figure 2-9: Schematic representation of the EC-SPR setup with a potentiostat for electrical control, laser light, polarizer, and half-wave retarder for p-polarized light. A detector, current amplifier, lock-in amplifier and oscilloscope were used for data collection.

A CW laser (Obis from Coherent) was deployed in combination with a half-wave plate and a linear polarizer to establish linearly polarized light at transverse-magnetic (TM) polarization (also known p-polarized light). The laser beam was directed towards the entrance facet of the coupling prism to excite the surface plasmon wave. A potentiostat (CHI 660D, CH Instruments, Inc.) was connected to the three electrodes to apply and control the potential at the working electrode. Two measurements were employed to characterize the EC-CPR device: In the first measurement, the SPR reflectance curves for 
a $500 \mathrm{nM}$ cytochrome c protein solution were determined at two applied potential (-0.3 and $0.3 \mathrm{~V}$ ) corresponding to the oxidized and the reduced states of cytochrome c protein, respectively. Second measurements were taken under cyclic voltammetry (CV) modulation. For these measurements, the electrically modulated reflected optical signal was monitored at a fixed angle by the power meter and transferred to an oscilloscope (Agilent, DSO8104A Infiniium).

\subsubsection{Immobilization and Characterization of Self-Assembled Monolayer onto EC-SPR Platform}

The molecular self-assembled monolayers (SAMs) of alkanethiols are widely used for modifying metal surfaces such as gold, platinum, and silver and have become a key element for building many systems and devices with applications in the wide field of nanotechnology [98-100]. Typically, the SAMs molecules contain three different parts: I) the head group (sulfur) which has a strong affinity for binding to metal surfaces like gold and silver, II) the thiol terminal groups like $\mathrm{COOH}, \mathrm{NH}_{2}$ and $\mathrm{OH}$ acting as a hydrophilic agent, or $\mathrm{CH}_{3}$ and $\mathrm{CF}_{3}$ as hydrophobic functional groups on the surface, and III) the backbone which connects the head groups to the terminal group [101, 102]. Soft proteins like bovine serum albumin, myoglobin, hemoglobin, and cytochrome c protein are strongly adsorbed on bare metal surfaces through hydrophobic or electrostatic interactions. The strong interaction between the soft proteins and a metal electrode leads to a conformational change or denaturation of the adsorbed proteins $[103,104]$. The use of SAMs on electrodes provides an effective method to protect the proteins from direct interaction with solid surfaces, and therefore avoids the denaturation of the protein and enhance the immobilized proteins' activity [105-108]. Following was the procedure for functionalizing the bilayered 
(silver/gold) platform with the 3-mercaptopropionic acid (MPA). The device interface of the bilayered (silver/gold) platform was sequentially cleaned in an ultrasonic bath with deionized (DI) water, acetone, and ethanol for 10 minutes in each solution, and then was dried under $\mathrm{N}_{2}$ gas. After those steps, the device was immersed into a $10 \mathrm{mM}$ solution of MPA in ethanol for $24 \mathrm{~h}$ to establish a self-assembled monolayer on the device interface. After rinsing the surface with ethanol and DI water, and drying it gently with $\mathrm{N}_{2}$ gas, the MPA- bilayered (silver/gold) platform was mounted on an electrochemical flow cell. The setup shown in Figure 2-9 was used first to stabilize the MPA-bilayered (silver/gold) platform with a PBS buffer solution under CV potential modulation from $-0.4 \mathrm{~V}$ to $0.2 \mathrm{~V}$ at a scan rate of $0.02 \mathrm{~V} / \mathrm{s}$. Then, the SPR reflectance curves for MPA-bilayered (silver/gold) platform on a PBS buffer were determined for each excitation wavelength. For calculated, the five-layer (BK7/silver/gold/MPA/PBS buffer solution) model was used. The optical constants of the gold and silver thin films were determined in the previous two sections, respectively. The measured and calculated SPR reflectance curves for MPA-bilayered (silver/gold) platform using the previously noted five excitation wavelengths are shown in Figure 2-10. 


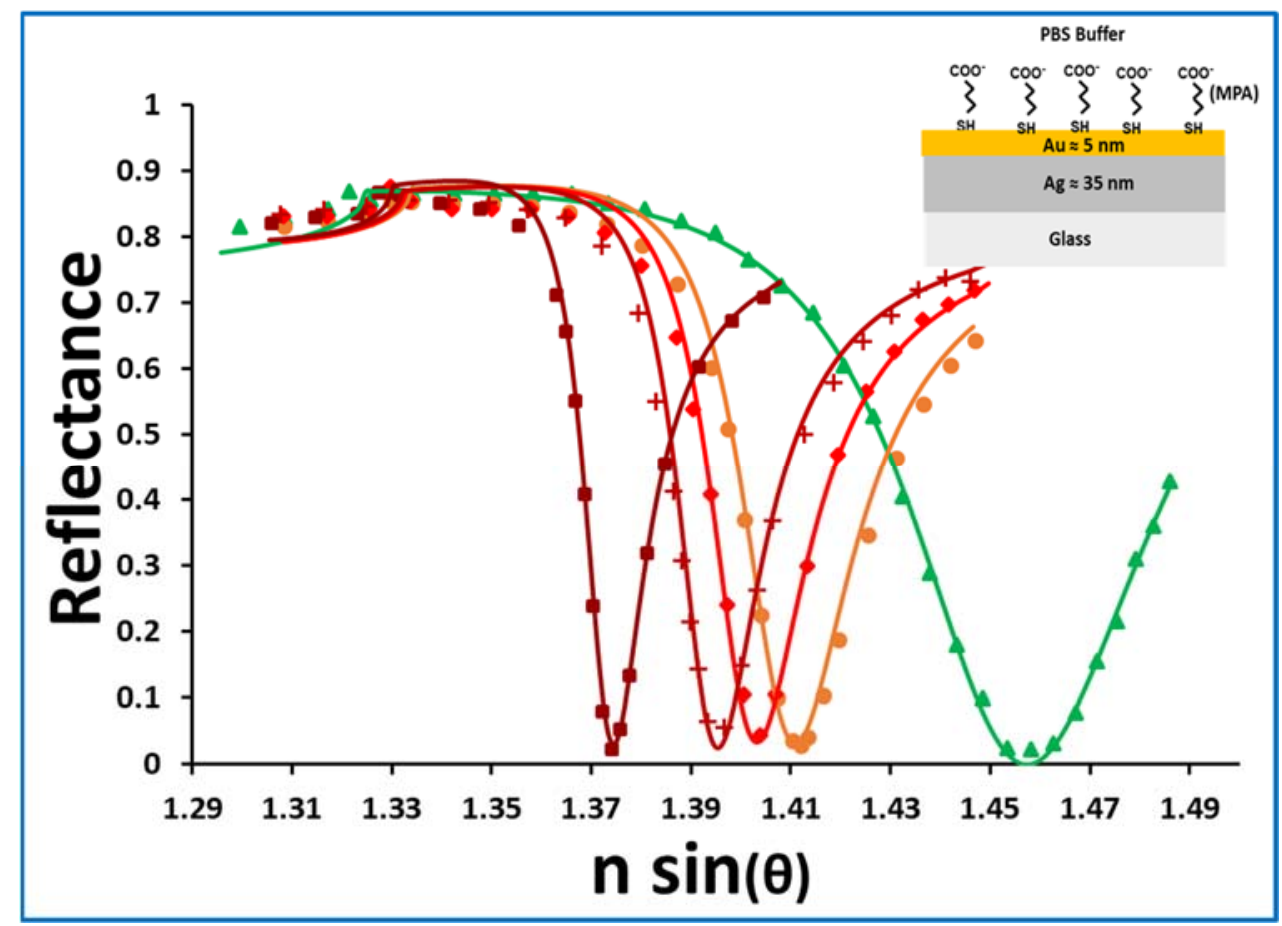

Figure 2-10: Experimental data for determination of the optical constants of the MPA layer in PBS buffer solution. The dotted curves are experimental results obtain by the conventional SPR setup at each excitation wavelength. The solid lines are calculated SPR reflectance curves. The wavelengths of light are $552 \mathrm{~nm}, 637 \mathrm{~nm}, 660 \mathrm{~nm}, 685 \mathrm{~nm}$, and $785 \mathrm{~nm}$.

The extinction coefficients of the MPA are assumed to be zero. The refractive index of the MPA layer at the five excitation wavelengths were extracted as previously described and listed in Table 2-3.

\subsubsection{EC-SPR Device Optimization}

The principle of the EC-SPR sensor relies on a strong difference, $\Delta R$, of the optical response between the reduced and oxidized states of redox-active probe. The cytochrome c protein redox active probe has been used as a model system. Two techniques were applied 
for searching for the ideal SPR configuration to optimize the EC-SPR response with the highest optical signal, $\Delta R$ : the conventional surface plasmon resonance (SPR) and the cyclic voltammetry (CV) technique.

\subsubsection{Surface Plasmon Resonance Reflectance Curves Measurements}

For full optimization tests of the EC-SPR device, the MPA-bilayered (silver/gold) platform was mounted into an electrochemical flow cell. A monolayer of cytochrome c protein was deposited onto the MPA-bilayered (silver/gold) platform by injecting a $50-\mu \mathrm{L}$ solution of cytochrome c protein at a concentration of $500 \mathrm{nM}$ in the flow cell and incubating for 30 minutes. Then, the flow cell was thoroughly rinsed with a PBS buffer solution to remove unbound species. The same setup, as in Figure 2-9 was used for three measurements: I) confirming the binding of the cytochrome $\mathrm{c}$ protein into the MPAbilayered (silver/gold) platform, II) determining the optical constants of cytochrome c protein at the two oxidation states, and III) optimizing of the EC-SPR device. In the first measurement, the SPR reflectance curves for a PBS buffer solution and for a layer of cytochrome c protein were determined using the five excitation wavelengths, as seen in Figure 2-11. 


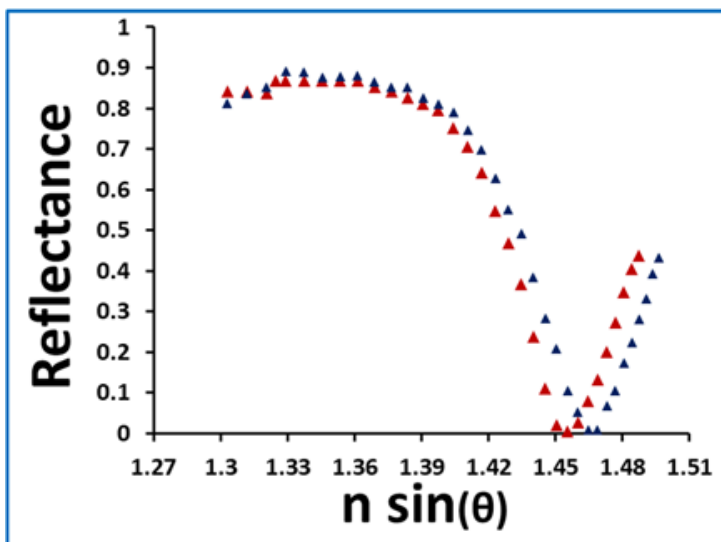

(c)

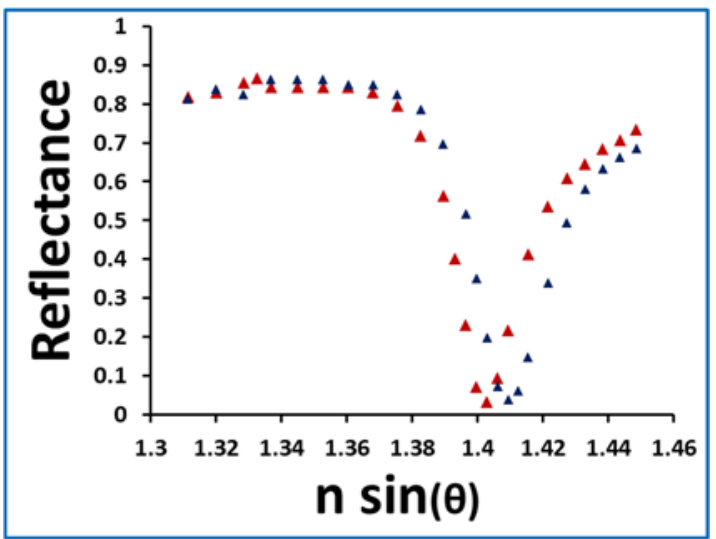

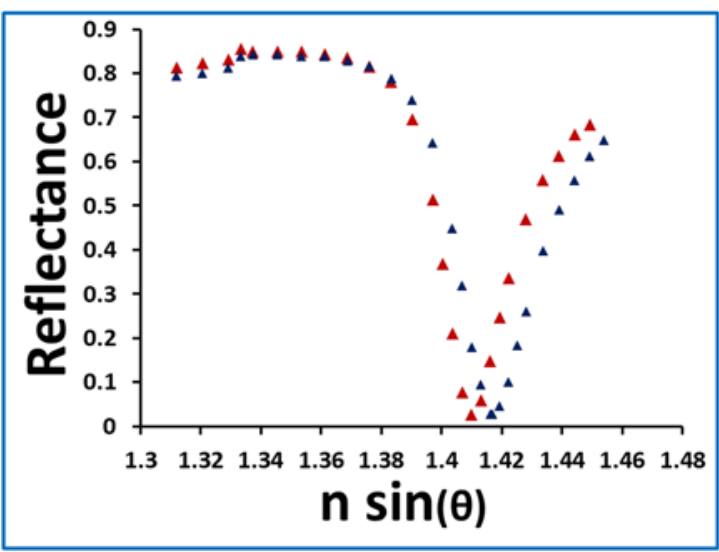

(d)

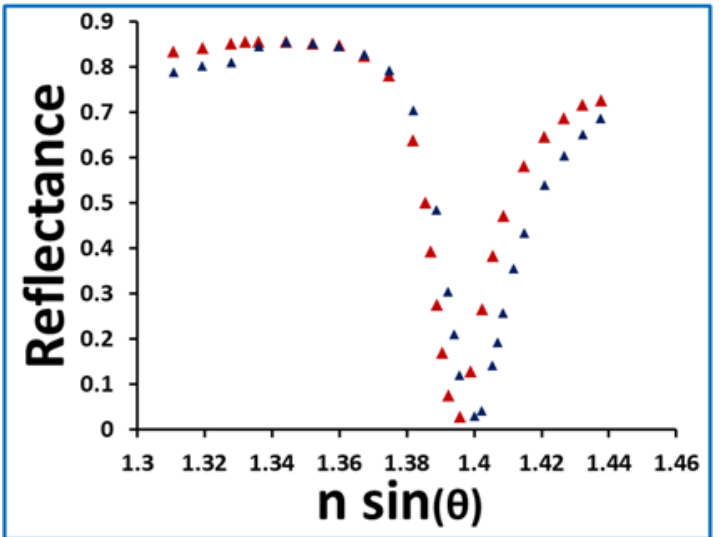

(e) 


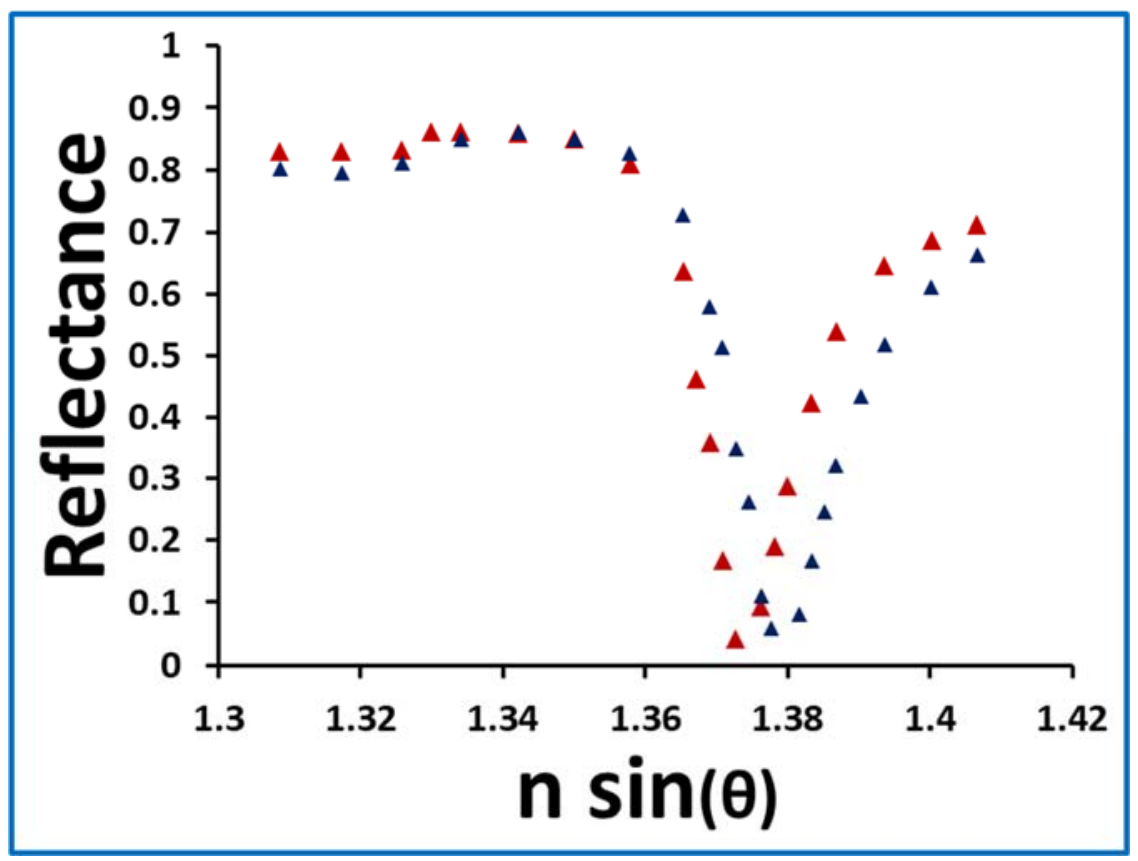

Figure 2-11: The experimental SPR reflectance curves of PBS buffer solution (red dots) and assembly of surface-bound cytochrome c protein (blue dots) using (a) $552 \mathrm{~nm}$, (b) 637 $\mathrm{nm}$, (c) $660 \mathrm{~nm}$, (d) $685 \mathrm{~nm}$, and (e) $785 \mathrm{~nm}$ excitation wavelengths.

The SPR dip shift (Figure 2-11) after introducing and rinsing the cytochrome c protein in the flow cell confirms the electrostatic binding between the cytochrome c protein and the MPA. After confirming the presence of the cytochrome c protein layer, the second measurement was carried out. Two electric potentials were applied respectively to control the oxidation states and therefore the optical response of the cytochrome c protein: $-0.3 \mathrm{~V}$ (which corresponds to a reduced state of cytochrome c protein) and $0.3 \mathrm{~V}$ (an oxidized state of cytochrome c protein). The SPR reflectance curves for each oxidation state were measured. Calculated results based on a Mathematica program were used to match the measured SPR reflectance curves to determine the optical constants of the cytochrome c protein in both oxidized and reduced states. For the calculated, the six-layer (BK7/silver/gold/MPA/cytochrome c protein/PBS buffer solution) model was used. Figure 
2-12 shows the measured and calculated SPR reflectance curves against the effective refractive index inside the prism, $n \sin (\theta)$, using the five excitation wavelengths.

(a)

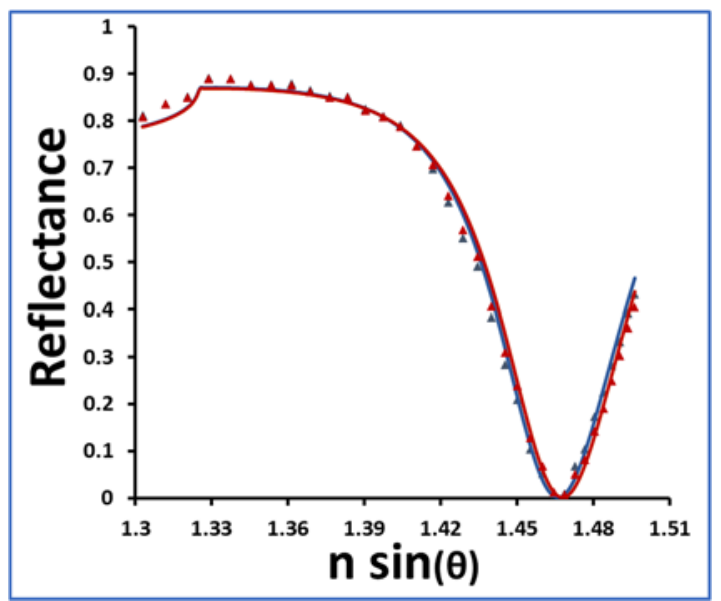

(c)

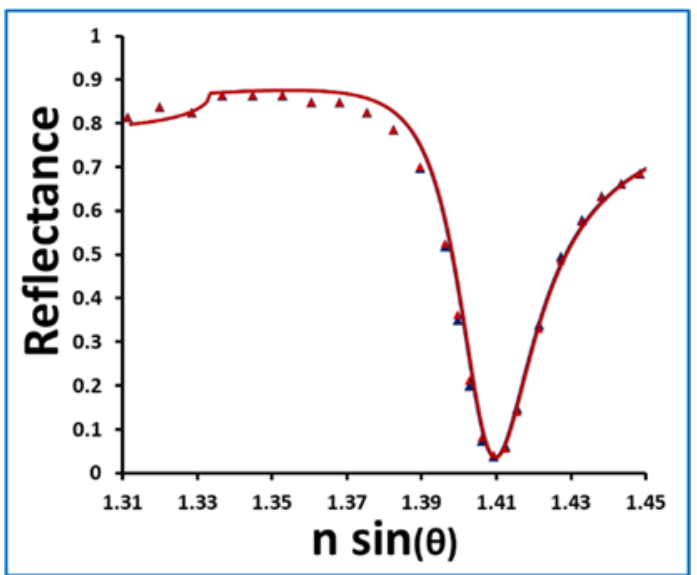

(b)

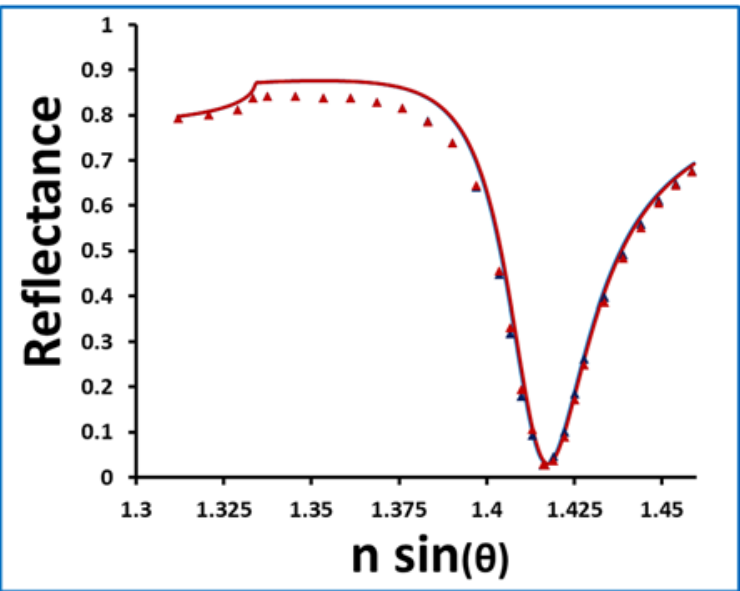

(d)

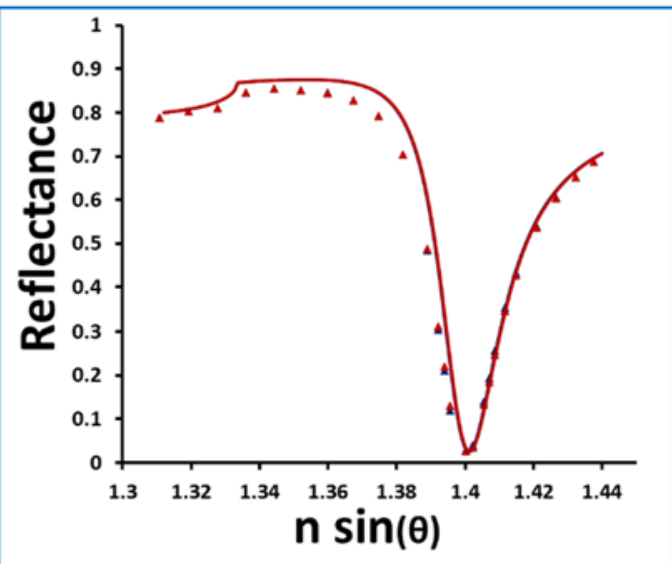

(e) 


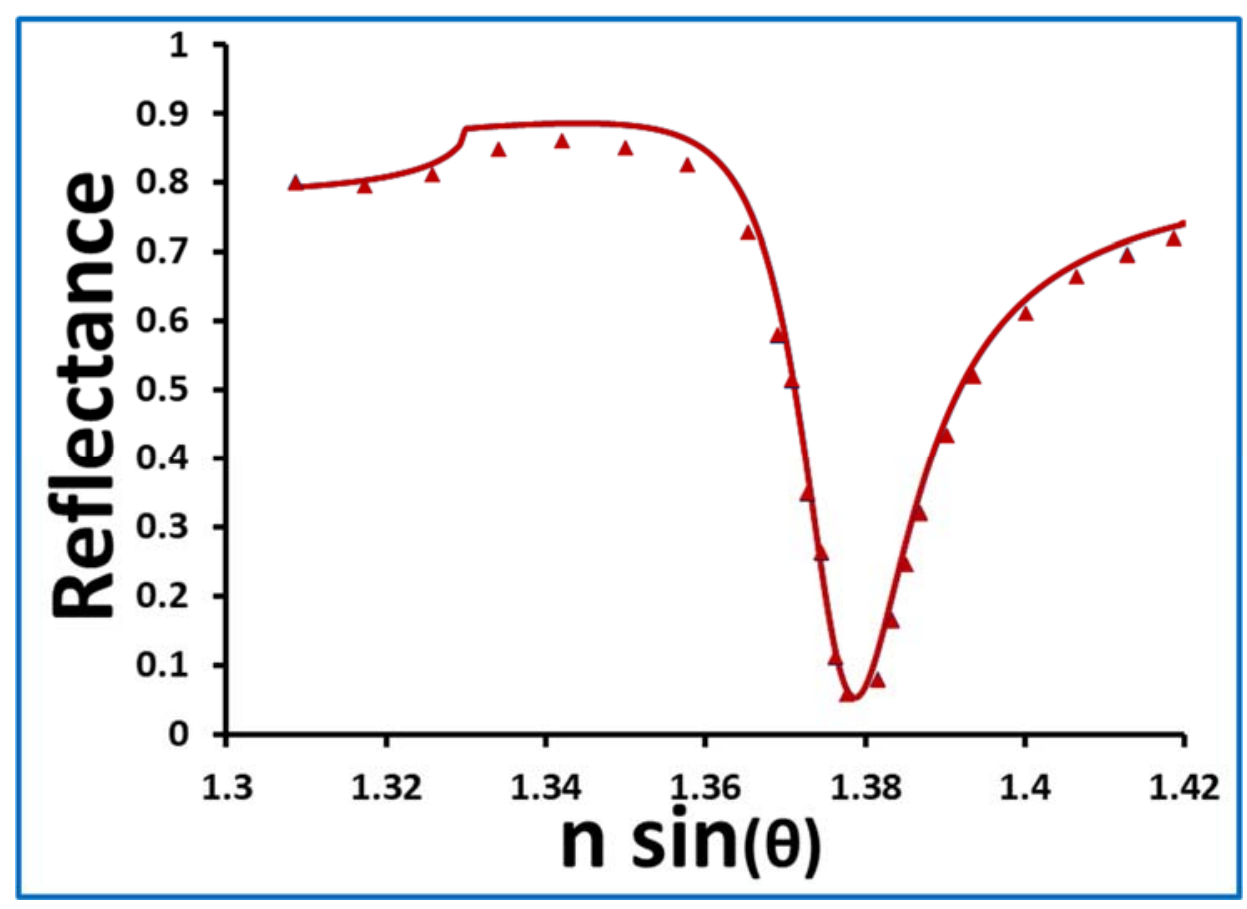

Figure 2-12: The optical constants examination of the cytochrome c layer in PBS buffer. The blue dotted curves are the experimental results obtained by the EC-SPR setup at -0.3 $\mathrm{V}$, while the red dotted curves are at $0.3 \mathrm{~V}$. The blue and red solid curves are the calculated SPR reflectivity curves using six-layer model (KB7/silver/gold/MPA/cytochrome c protein/PBS buffer) at $-0.3 \mathrm{~V}$ and $0.3 \mathrm{~V}$, respectively with (a) $552 \mathrm{~nm}$, (b) $637 \mathrm{~nm}$, (c) 660 $\mathrm{nm}$, (d) $685 \mathrm{~nm}$, and (e) $785 \mathrm{~nm}$ excitation wavelengths.

The optical constants of the cytochrome c protein at the two applied potentials were determined and listed in Table 2-3.

Table 2-3: Optical constants of the cytochrome c protein film were extracted by minimizing the difference between the measured SPR reflectance curves and the calculated curves. 


\begin{tabular}{|c|c|c|c|c|c|}
\hline $\begin{array}{c}\text { Wavelength } \\
(\mathrm{nm})\end{array}$ & MPA & \multicolumn{2}{|c|}{ Oxidized cytochrome c } & \multicolumn{2}{c|}{ Reduced cytochrome c } \\
\hline & $\mathrm{n}$ & $\mathrm{n}$ & $\mathrm{k}$ & $\mathrm{n}$ & $\mathrm{k}$ \\
\hline 552 & 1.5313 & 1.5255 & 0.0026 & 1.5655 & 0.0090 \\
\hline 637 & 1.5569 & 1.5789 & 0.0005 & 1.5909 & 0.00043 \\
\hline 660 & 1.5524 & 1.5793 & 0.00045 & 1.5937 & 0.00035 \\
\hline 685 & 1.5420 & 1.5359 & 0.00045 & 1.5470 & 0.00035 \\
\hline 785 & 1.4839 & 1.6140 & 0.00023 & 1.6904 & 0.00038 \\
\hline
\end{tabular}

The results in Table 2-3 show that both the real and imaginary parts of the refractive index of the cytochrome $\mathrm{c}$ protein are modified when undergoing a redox process. Then to verify these results, a conventional UV-vis spectrophotometer $(400-800 \mathrm{~nm})$ was used. The molar absorptivity of the cytochrome c protein in the PBS buffer solution at the two oxidation states was investigated. First, the molar absorptivity for oxidized cytochrome c protein in PBS buffer solution was determined. Then, the oxidized cytochrome c protein were chemically reduced with sodium dithionite $(0.29 \mathrm{M})$ at room temperature in PBS buffer solution and the molar absorptivity was determined, as seen in Figure 2-12. Since the imaginary part of the refractive index, $k$, is directly proportional to the molar absorptivity, the imaginary parts of the refractive index for cytochrome c protein, $k$, obtained by the SPR reflectance curves were plotted in the same graph (Figure 2-12). 


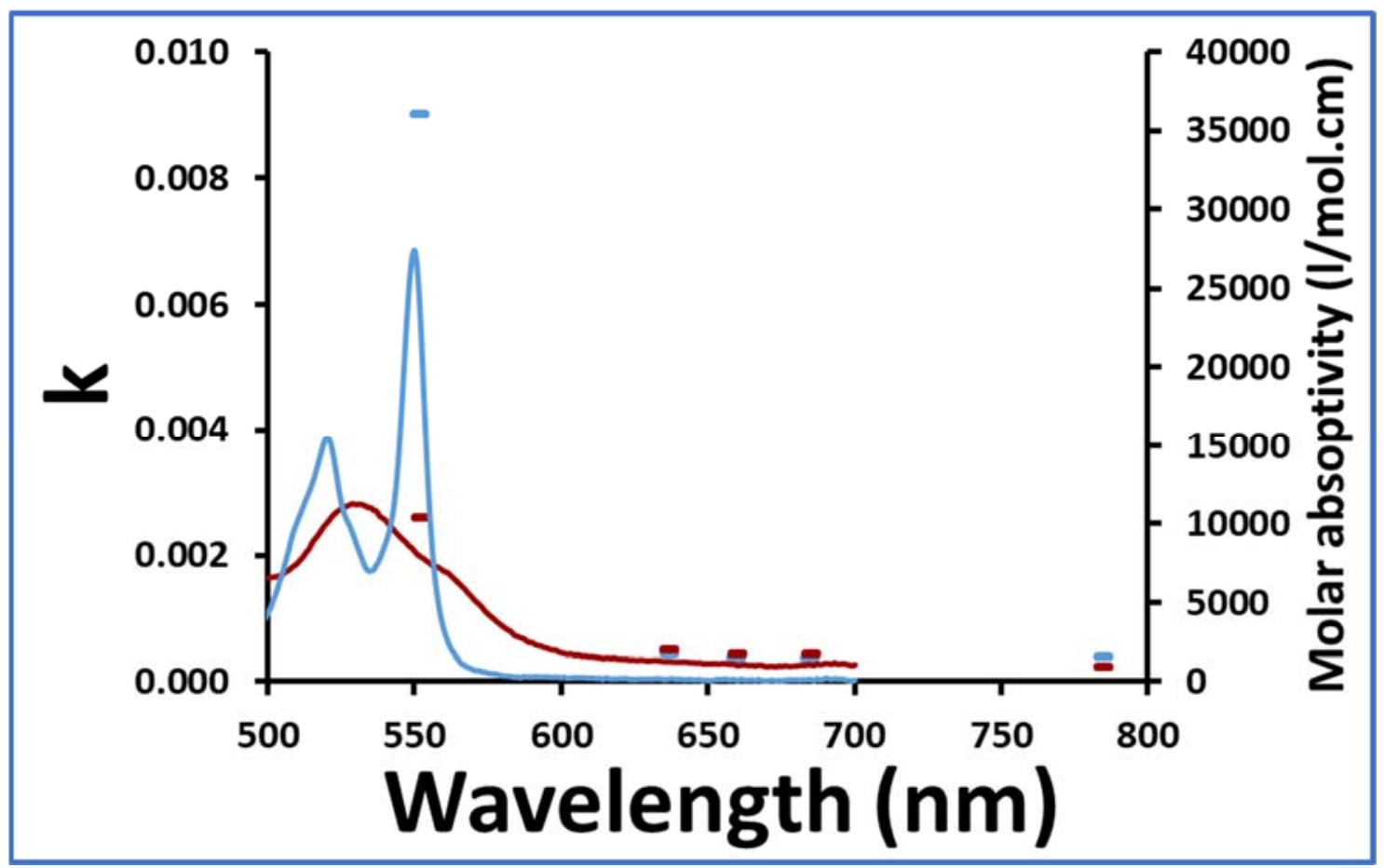

Figure 2-13: Right y-axis shows the molar absorptivity for the oxidized (red curve) and the reduced states (blue curve) of the cytochrome c protein in PBS buffer solution measured by UV-vis spectrophotometer, while the left y-axis shows the imaginary part of the refractive index of the cytochrome c protein, $k$, at oxidized state (red dotted curve) and reduced state (blue dotted curve) obtained by the SPR reflectance curves.

As seen in Figure 2-13, the trend of the experimental values of the imaginary part of the refractive index, $k$, versus the wavelength is in high agreement with the molar absorptivity for the cytochrome c protein in PBS buffer solution at the two oxidation states.

In the third measurement, the changes in both the real and imaginary parts of the refractive index of the cytochrome c protein when undergoing a redox process were used to define the best fixed angle (i.e., ideal effective refractive-index) to implement EC-SPR measurements that will maximize the amplitude of the optical modulation when an electrochemical modulation is applied. First, the effect of the background (the absence of 
the cytochrome $\mathrm{c}$ protein in the flow cell) on the optical signal difference, $\Delta R$, was examined. The SPR reflectance curves were determined at the two applied potential values $(0.3 \mathrm{~V}$ and $-0.3 \mathrm{~V})$ with the presence of the PBS buffer solution in the flow cell and then the optical signal difference, $\Delta R$, was calculated. Next, the SPR reflectance curves of the cytochrome c protein in Figure 2-12 were used to determine the optical signal difference, $\Delta R$, of the cytochrome c protein solution at each excitation wavelength. The results plotted in Figure 2-14 shows the optical signal difference, $\Delta R$, in the presence (green, light orange, dark orange, light red, and dark red) and absence (light blue data points) of the cytochrome c protein in the flow cell at each excitation wavelength.

(a)

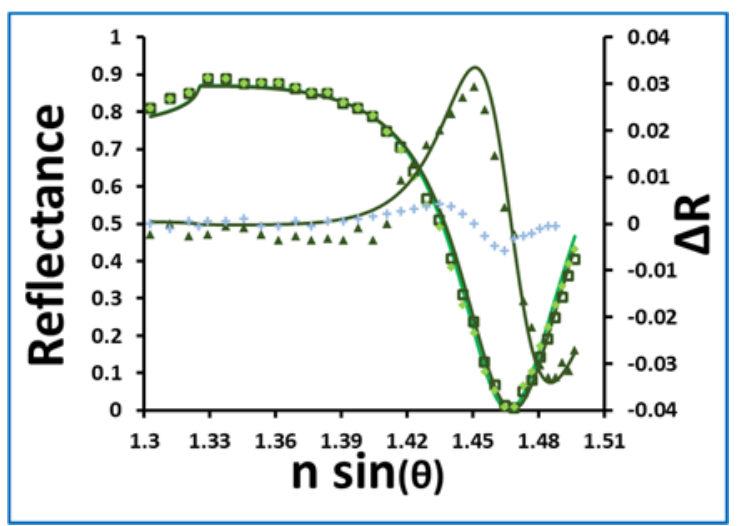

(c)

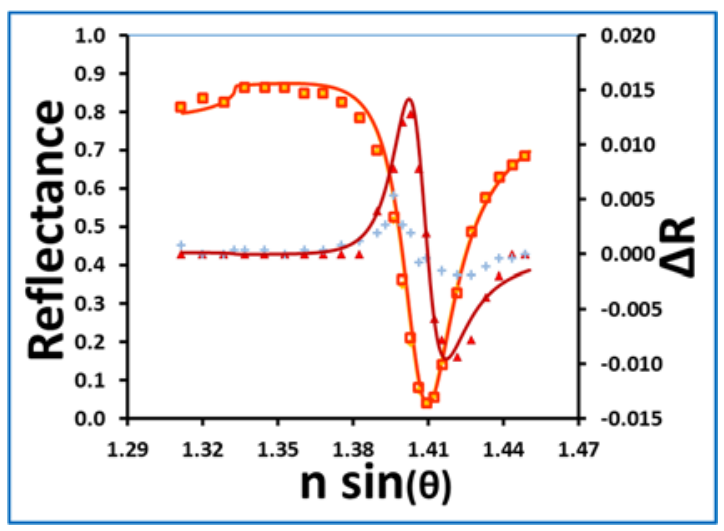

(b)

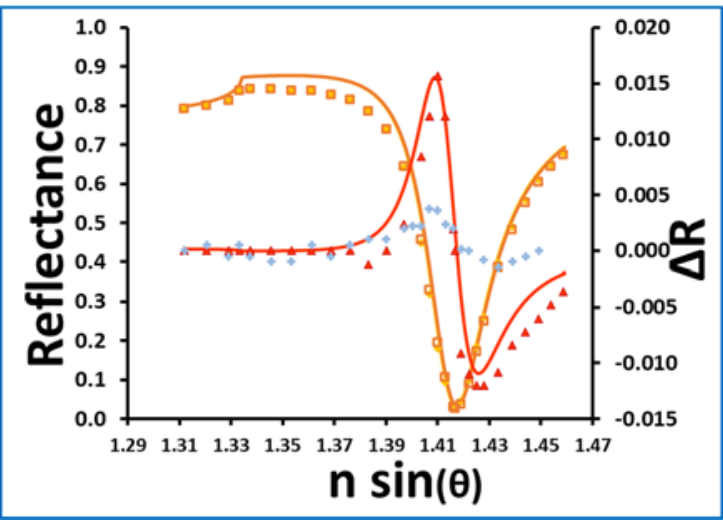

(d)

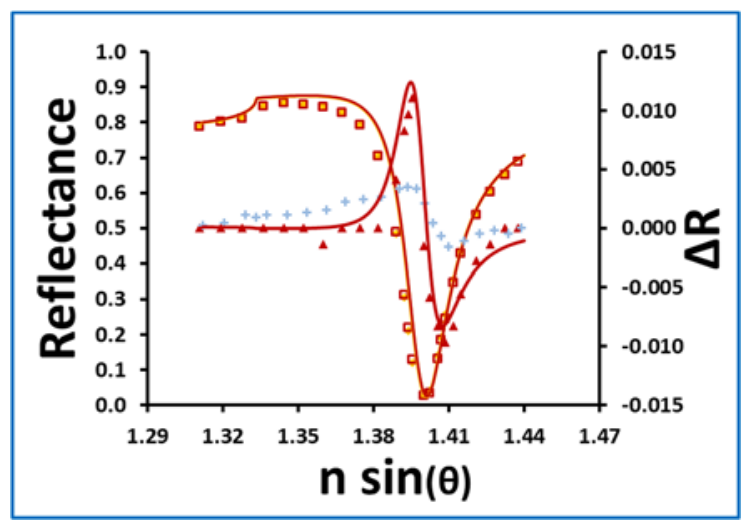


(e)

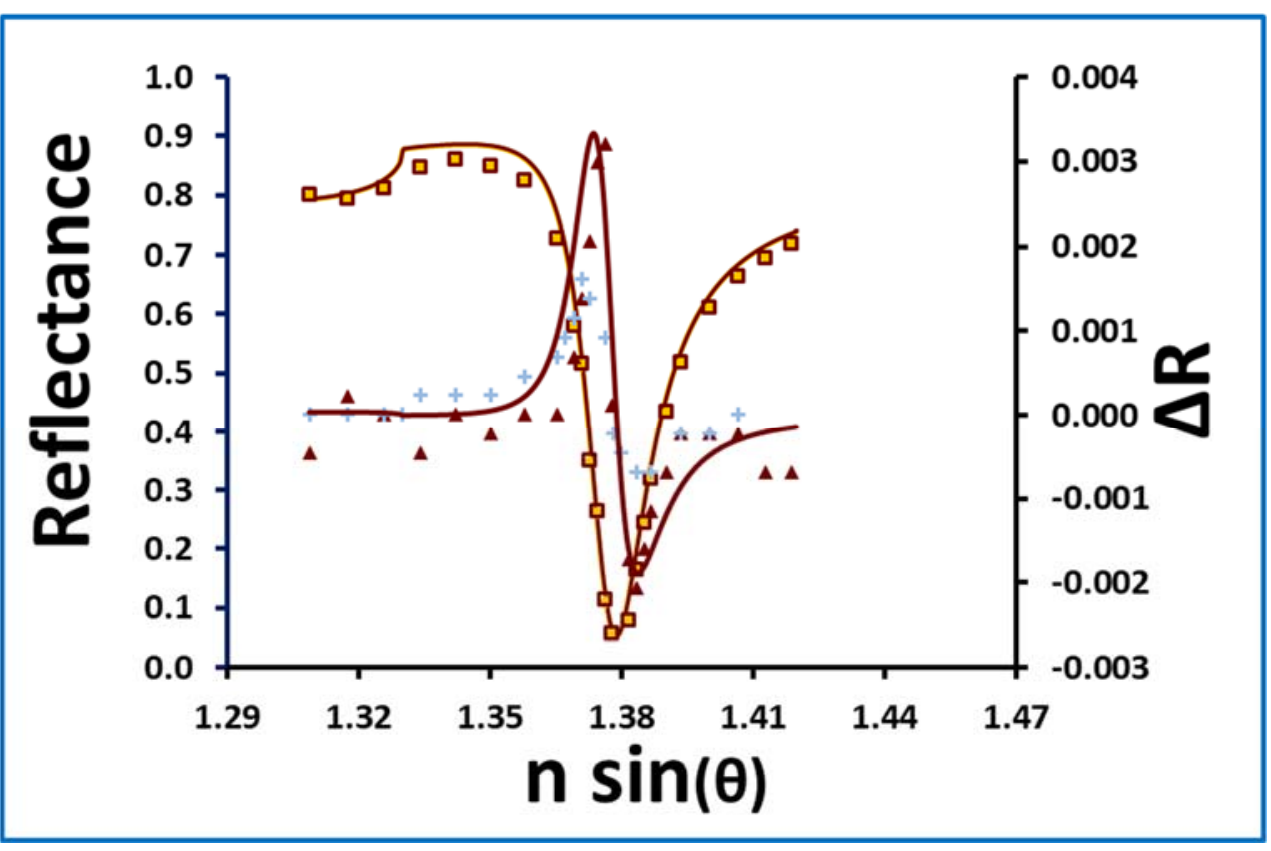

Figure 2-14: Left y-axis shows the experimental SPR reflectance curves of assembly of surface-bound cytochrome c protein at oxidized and reduced states (the dotted curves) and the calculated data at the same two oxidation states (the line curves), while right y-axis shows the measured , $\Delta R$, in the presence (the dotted curves) and absence (light blue data points) of the cytochrome c protein in the flow cell, and the calculated, $\Delta R$, (the line curves) in the presence of the cytochrome c protein in the flow cell with (a) $552 \mathrm{~nm}$, (b) $637 \mathrm{~nm}$, (c) $660 \mathrm{~nm}$, (d) $685 \mathrm{~nm}$, and (e) $785 \mathrm{~nm}$ excitation wavelengths.

As shown in Figure 2-12, I) in the absence of the cytochrome c protein in the flow cell (light blue data points), the optical signal difference, $\Delta R$, which is due to applied potential effects on the surface plasmon wave, is small compared to those in the presence of the cytochrome $\mathrm{c}$ protein and can be neglected. II) The highest magnitude of the, $\Delta R$, is observed to occur in the linear region on the left side of the resonant angle of the conventional SPR curve. III) The experimental data of $\Delta R$ are in excellent agreement with 
the calculated data at each wavelength. Then, the experimental and calculated optical signal difference, $\Delta R$, were plotted against the effective refractive index inside the prism, $n \sin (\theta)$, at $552 \mathrm{~nm}, 637 \mathrm{~nm}, 660 \mathrm{~nm}, 685 \mathrm{~nm}$ and $785 \mathrm{~nm}$ excitation wavelengths, Figure 2-15.

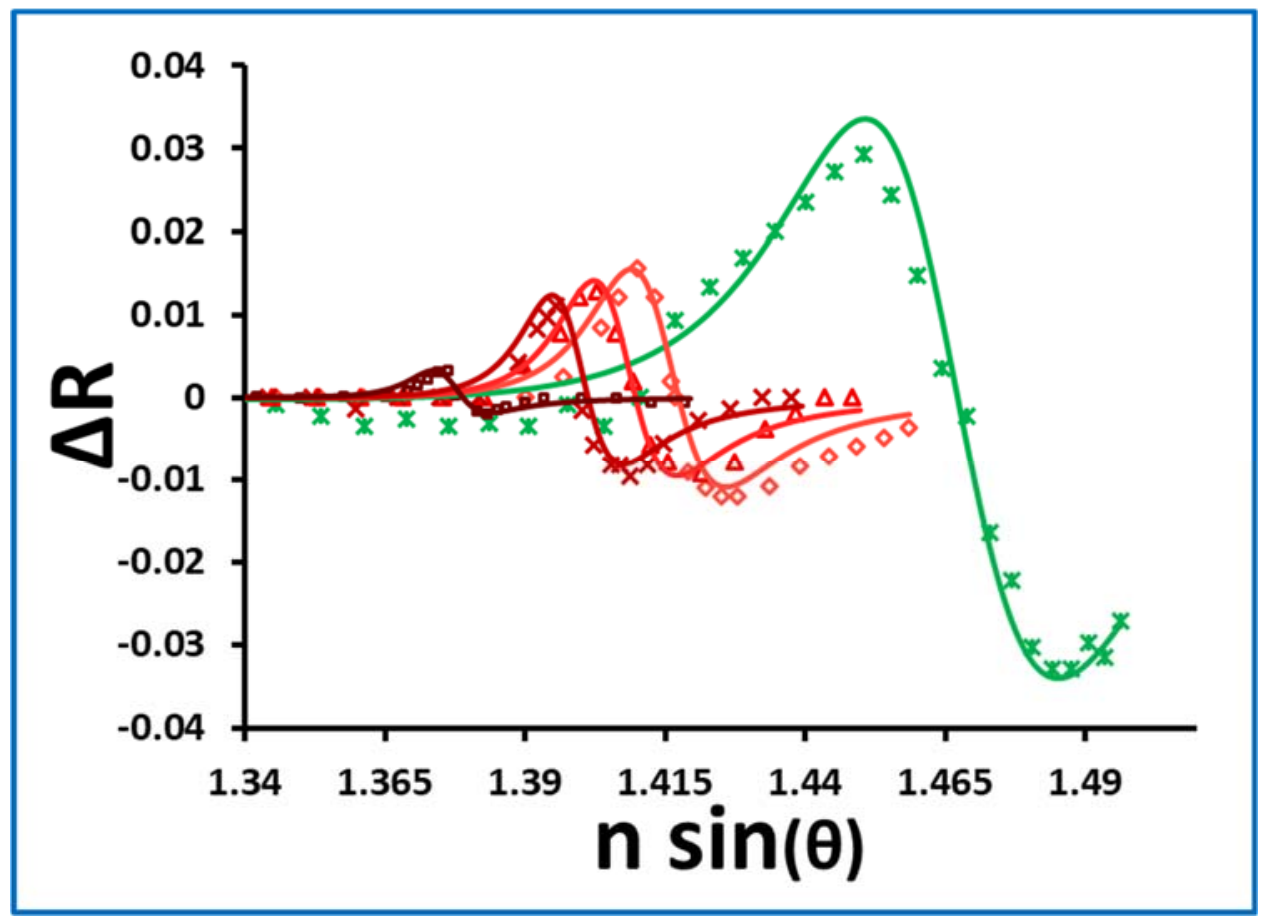

Figure 2-15: The signal difference $\Delta R$ between two applied potentials against the effective refractive index inside the prism, $n \sin (\theta)$, at $552 \mathrm{~nm}, 637 \mathrm{~nm}, 660 \mathrm{~nm}, 685 \mathrm{~nm}$ and 785 nm excitation wavelengths. The dotted curves are the experimental data, whereas the line curves are the calculated data based on experimentally-determined optical constants.

The experimental and calculated curves show that the highest magnitude of $\Delta R$ is obtained when the excitation wavelength was $552 \mathrm{~nm}$, where the $\Delta R$ relies on the highest change in both the real and imaginary parts of the refractive index of the cytochrome $\mathrm{c}$ protein when undergoing a redox process. Since the iron centered in the heme-group of the cytochrome c protein is responsible for both the electronic redox exchange and the optical transition, and observing that such spectral transition is localized near $550 \mathrm{~nm}$, we can conclude that 
tuning the excitation wavelength to the absorption transition of the redox probe will maximize the amplitude of the optical modulation when an electrochemical modulation is applied.

\subsubsection{Cyclic Voltammetry (CV) Measurements}

The preliminary results of section 2.3.4.1, which were obtained for two fixed values of the applied potential, are further expanded here by the cyclic voltammetry (CV) technique that provides a continuous linear sweep of the electrical potential over a welldefined range. The same experiment setup as seen in Figure 2-9 was used in the CV measurements. A monolayer of cytochrome c protein was deposited onto the MPAbilayered (silver/gold) platform by injecting a $50-\mu \mathrm{L}$ solution of cytochrome c protein at a concentration of $500 \mathrm{nM}$ in the flow cell and incubating for 30 minutes. Then the flow cell was thoroughly rinsed with a PBS solution to remove unbound species. The monolayer of cytochrome $\mathrm{c}$ protein is immobilized on the platform surface by electrostatic binding to the MPA. CV potential modulation from 0 to $0.35 \mathrm{~V}$ at a scan rate of $0.02 \mathrm{~V} / \mathrm{s}$ was used to confirm the monolayer coverage. The electrical current data measured by the potentiostat are shown in Figure 2-16.

(a)

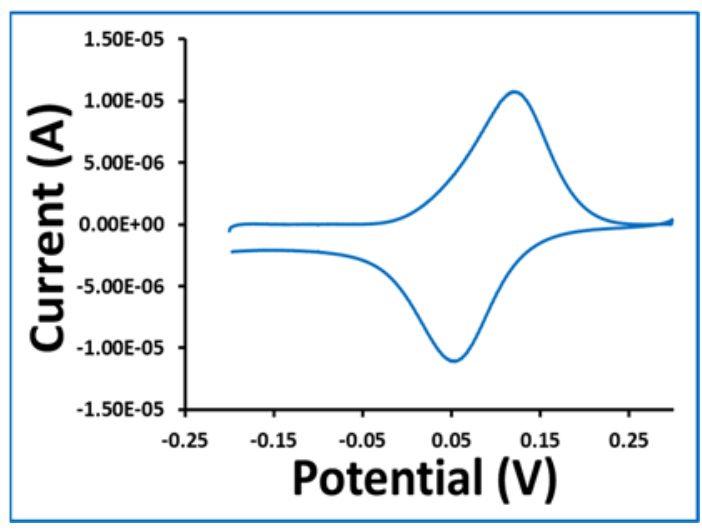

(b)

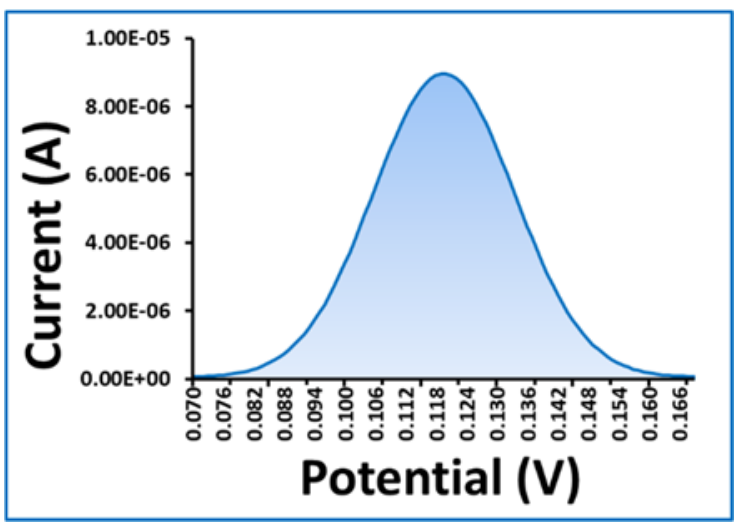


Figure 2-16: (a) Cyclic voltammetry with cytochrome c protein immobilized onto the MPA-bilayered (silver/gold) platform with scan rate of $0.02 \mathrm{~V} / \mathrm{s}$. (b) The redox peak area.

Clear faradaic current density peaks were identified for cytochrome c protein adsorbed onto the MPA-modified platform. The surface coverage was determined by measuring the reduction peak area and applying the flowing Equation $[109,110]$ :

$$
\Gamma=\frac{Q}{n F A}
$$

Where $\mathrm{Q}$ is the charge associated with the anodic oxidation of cytochrome c protein and is measured by integration of the charge current over the reduction peak, $n$ is the number of electrons in the electron-transfer process (for the cytochrome c protein $n=1$ ), $F$ is Faraday's constant and $A$ is the area of the electrode. Figure 2-14 (b) shows the reduction peak integration, where the background due to non-faradaic components displayed in Figure 2-14 (a) has been removed. The surface coverage of cytochrome c protein electrostatically adsorbed onto the MPA-modified platform was measured to be 11.66 $\mathrm{pmol} / \mathrm{cm}^{2}$. The measured value of the surface coverage is quite consistent with the theoretical calculations for cytochrome c protein directly adsorbed to the MPA-modified gold surface [15].

After confirming the cytochrome $\mathrm{c}$ protein layer adsorption on top of the MPA-bilayered (silver/gold) platform, a series of potential scans from $-0.3 \mathrm{~V}$ to $0.3 \mathrm{~V}$ at a scan rate of 0.02 $\mathrm{V} / \mathrm{s}$ were performed for the optical interrogation. Three values the effective refractive index inside the prism, $n \sin (\theta)$, were examined for each excitation wavelength. Figure 2-15 shows the measured reflectance against the effective refractive index inside the prism, $n$ $\sin (\theta)$, for $552 \mathrm{~nm}$ excitation wavelength. The results using $637 \mathrm{~nm}, 660 \mathrm{~nm}, 685 \mathrm{~nm}$ and $785 \mathrm{~nm}$ are shown in Appendix A-9. 
(a)

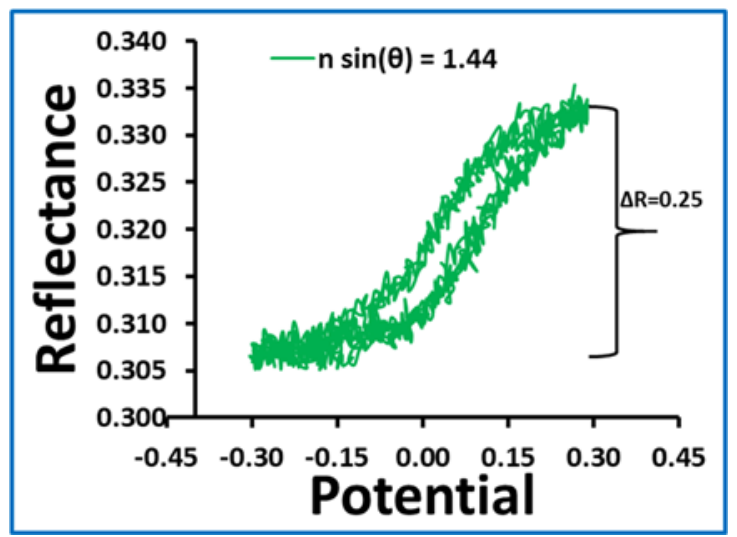

(b)

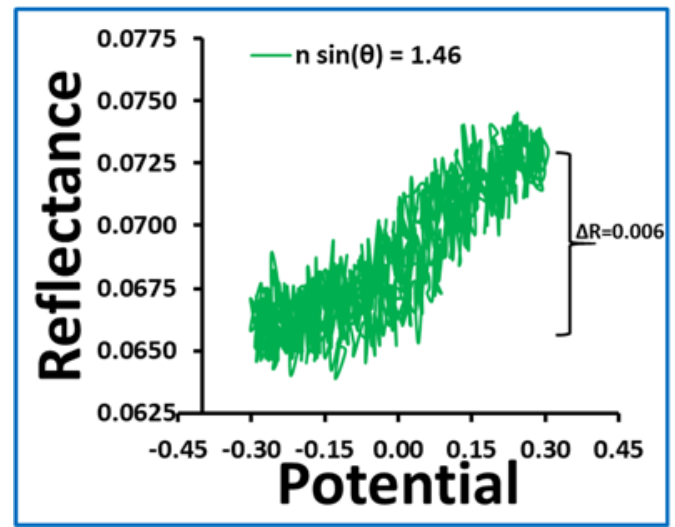

(c)

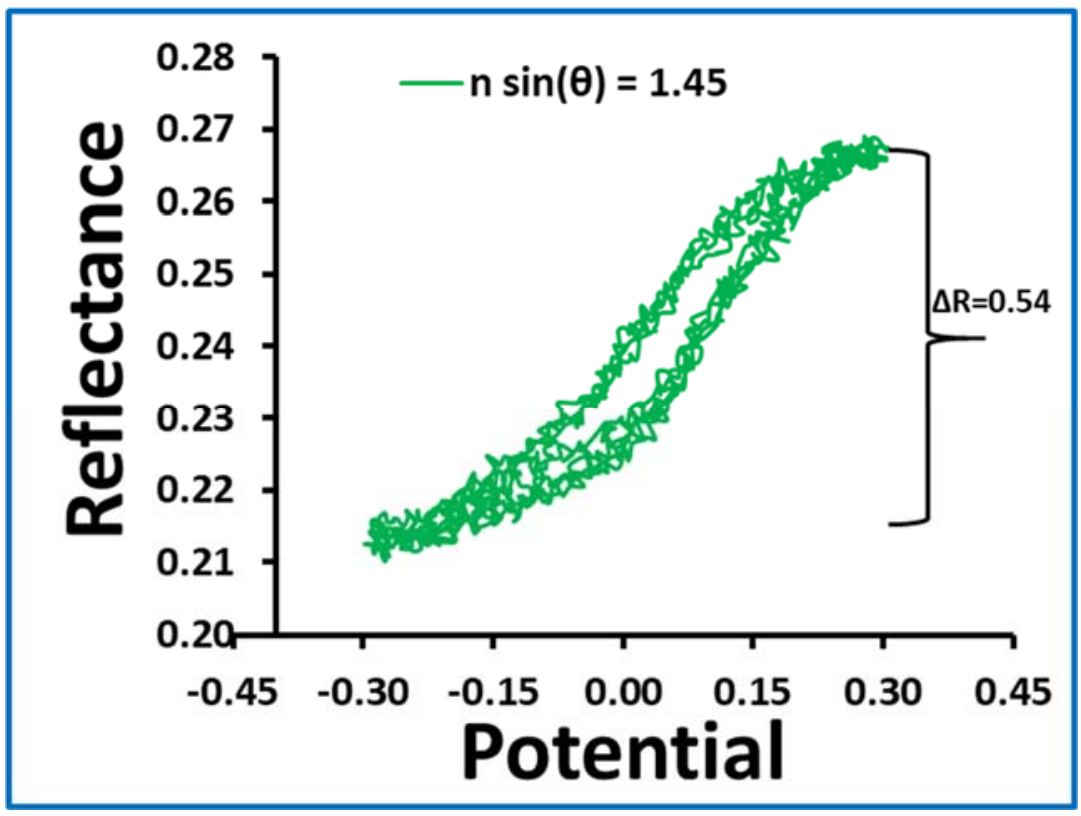

Figure 2-17: Reflectance under a CV scan for the cytochrome c protein immobilized onto MPA-bilayered (silver/gold) platform with scan rate of $0.02 \mathrm{~V} / \mathrm{s}$ at (a) $n \sin (\theta)=1.44$, (b) $n \sin (\theta)=1.46$, and (c) $n \sin (\theta)=1.45$.

The results summarized in (a), (b), and (c) of Figure 2-14 show a reversible change in the reflectance as the modulation potential crosses the formal potential of the cytochrome c protein (approximately at $0.05 \mathrm{~V}$ ). From these data, the $\Delta R$ were estimated to be 0.54 for $n$ $\sin (\theta)=1.45,0.25$ for $n \sin (\theta)=1.44$, and 0.006 for $n \sin (\theta)=1.46$. The same trend was 
observed using the other excitation wavelengths (Appendix A-9). These results show that the magnitude of the useful reflectance, $\Delta R$, at certain excitation wavelengths were highly dependent on the effective index, $n \sin (\theta)$, used to optically interrogated the protein layer. Moreover, the highest magnitude of the $\Delta R$ was observed in the linear region on the left side of the resonant angle of the conventional SPR curve. Next, in order to find the dependence of $\Delta R$ on the excitation wavelength, the $\mathrm{CV}$ measurements at the optimal effective index $n \sin (\theta)$ that give the highest magnitude of $\Delta R$ for the five excitation wavelengths are plotted in one graph, Figure 2-16.

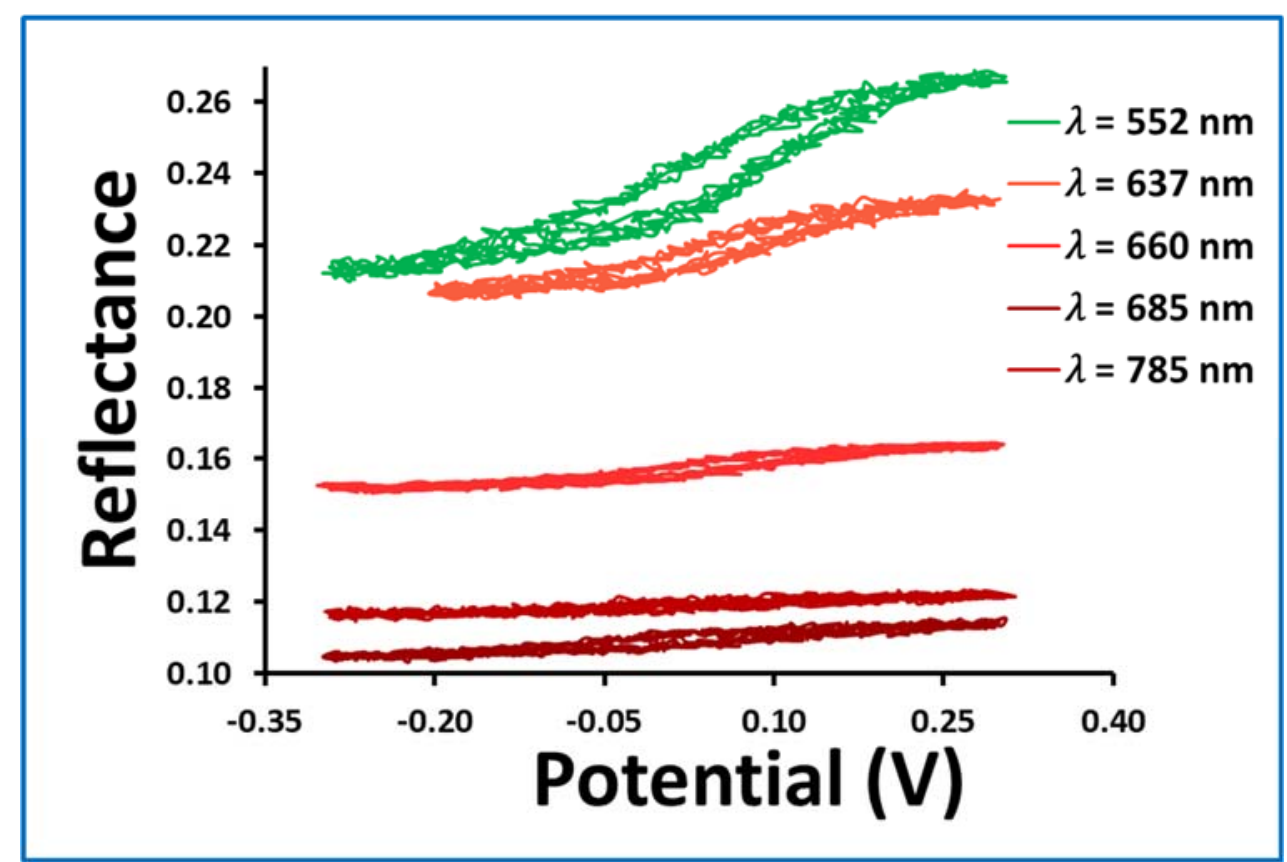

Figure 2-18: Reflectance under a CV scan for the cytochrome c protein immobilized onto MPA-bilayered (silver/gold) platform with scan rate of $0.02 \mathrm{~V} / \mathrm{s}$ and using the optimal effective index $n \sin (\theta)$ at each wavelength.

These results demonstrate that the highest magnitude of the useful $\Delta R$ was observed for the excitation wavelength $552 \mathrm{~nm}$ (green trace), whereas the smallest $\Delta R$ was observed when the 685 and $785 \mathrm{~nm}$ wavelengths were used as an excitation wavelength. These results are 
in excellent agreement with the results determined in the previous section. As a result, $\Delta R$ is largely affected by the $n \sin (\theta)$ and the excitation wavelength, therefore defining the optimal effective index and wavelength to implement EC-SPR measurements is an effective way to enhance the analytical signal and increase the sensitivity of EC-SPR device.

\subsection{Conclusions}

In this study, a redox probe featured by the cytochrome $\mathrm{c}$ protein was used as a model system to illustrate our rational formalism to proper define the optimal parameters to optically interrogate the EC-SPR device aiming to maximize the optical response associated with the faradaic redox process. Our results show that the excitation wavelength and the effective refractive index, $n \sin (\theta)$, are the main parameters effecting $\Delta R$ under potential modulations. Moreover, we found that the interrogating the EC-SPR device in the linear region of the resonant angle of the conventional SPR curve and tuning the excitation light to the absorption band of any redox probe leads to maximum $\Delta R$ under potential modulation and therefore enhances the sensitivity of detection. Obviously, the methodology described above needs to be replicated when modifications are made in the photonic platform, as for instance in the deployment of a new redox probe or of a new metal-film structure for carrying the plasmon surface wave. After the development and the optimization of the EC-SPR device for establishing a superior performance and successfully adapted for spectroelectrochemical measurements, the device is ready for deployment with the AC potential modulation for studying the impact of different functionalizing layers and immobilization strategies on the electron-transfer rate of the surface-adsorbed redox species. We anticipate that with EC-SPR improvements, the device 
sensitivity in the study of surface-confined redox species at low surface densities can be increased to overcome the effects of background signals in biosensor applications. 


\section{CHAPTER 3}

\section{ELECTRON-TRANSFER KINETICS OF REDOX PROBE AT}

\section{DIFFERENT MOLECULAR ASSEMBLIES}

\subsection{Introduction}

The electron-transfer of redox proteins is a fundamental biological process that controls the physical properties and chemical activities of molecular and biological systems [111-113]. Understanding and controlling the electron-transfer mechanism through biomolecular assemblies at modified electrode surfaces has a crucial impact in many applications such as bio-electronics devices, bio-solar cells, and health science researches[114-119]. Over the years, the electron-transfer process in molecular assemblies at electrode surfaces has been the subject of substantial theoretical and experimental studies. The electron-transfer mechanism [120], the distance dependence of the electrontransfer rate constants $[113,121]$, and the effect of orientation and conformational on the electron-transfer rate constants[122, 123] have been extensively studied and reported. However, some applications, such as biosensors and biofuel cells, require well-designed strategies for immobilization of molecules onto the surfaces in order to have a direct and fast electron-transfer between the biological system and the electrode surface. SAM's are

one means of modifying the electrode surface and were used to immobilize proteins through a covalent attachment, hydrophobic interactions and electrostatic interaction [124126]. Thus, it provides an effective method to protect the proteins from the denaturation 
and then enhance the electron-transfer between the redox protein of interest and the electrode surface[105-108]. The traditional electrochemical techniques (amperometric, voltammetric, and impedimetric) are the most used techniques for investigation electrochemical processes for surface-immobilized redox species[127-132]. However, the sensitivities of traditional electrochemical techniques that rely solely on electrical signals remain inadequate for critical physiological applications. Invariably, non-faradic components that arise from electric double-layer effects formed from the flow of electrolyte ions can hinder the detection of redox events at surfaces with low analyte concentrations. Therefore, the detection sensitivity is usually limited by a strong background of the non-faradic current[35]. A new analysis strategy based on an optical impedance spectroscopy technique to study and determine the electron-transfer rates for surface immobilized redox species was developed by Xan et al. [133]. The strategy is based on an $\mathrm{AC}$ potential modulation performed in order to drive an electrochemical redox process of a monolayer of redox molecules on an indium tin oxide (ITO)-based single-mode, electro-active, integrated optical waveguide (SM-EA-IOW) platform. Then, by combining the optical modulated absorbance and the electrical impedance data across the electrochemical cell, the electron transfer rate is determined. This strategy has been demonstrated experimentally to provide a straight forward approach to determine the electron transfer rate of the surface-adsorbed redox species at sub-monolayer level without the need to model and determine each component present in the equivalent electrical electrochemical cell. However, two important hurdles in the implementation of the SMEA-IOW optical impedance spectroscopy are I) the fabrication challenge of the ITO platform that simultaneously needs extremely high optical transparency for the propagation 
of guided waves and outstanding electrical conductivity, and II) the inability to implement the experimental strategies at much higher modulation frequencies and broad potential ranges due to the instability of ITO platform at high frequency and high applied potentials. However, in order to overcome these limiting factors and enabling measurements at a broader potential range, and much higher modulation frequencies to detect faster reaction rate constants of a wide range of surface-adsorbed redox species, the EC-SPR device combined with impedance spectroscopy techniques can be advantageous.

In this chapter, the theoretical framework developed by Xan et al. [133] is updated and applied with EC-SPR device. The same protocol is used for retrieving full electrochemical information from pure optical signals under potential modulation. A redox-active probe, cytochrome c protein has been used as a model system to demonstrate the capability of the EC-SPR device for determining the electron-transfer rate of a molecular assembly. Furthermore, the impact of different functionalized layers of the plasmonic interface and immobilization strategies (direct adsorption, electrostatic adsorption, covalent adsorption, and affinity adsorption) on the formal potential as well as on the electron-transfer rate are examined. Finally, the effect of adding bovine-serum albumin (BSA) onto the layers is examined. The impact of each interface of a multilayer assembly on the formal potential and the electron-transfer rate of bound cytochrome $\mathrm{c}$ protein is reported in this chapter.

\subsection{Theory for the Optical Impedance Spectroscopy Technique (OIS) with the EC-} SPR Device

The methodology of using the EC-SPR platform with OIS to investigate the electrontransfer rate of redox events is described here. An electrical potential is applied onto the 
metallic SPR interface while the light reflectivity is monitored at a properly-determined incident angle and at a well-chosen specific wavelength. By then setting a DC bias potential to a particular formal potential of redox species adsorbed onto the plasmonic interface and by applying an AC-modulated potential to force the oscillation of those redox species between their oxidized and reduced states, a modulated optical signal in reflection will be created[134]. Monitoring the magnitude and phase of such modulated optical signal with a lock-in amplifier can provides kinetic information about rates of electron transfer of the surface adsorbed species when they are switching between the reduced and oxidized states. In the OIS technique and under small amplitudes of an $\mathrm{AC}$ potential modulation, the relation between the applied potential and the modulated optical signal is given by: $E=$ $E_{d c}+\Delta E_{a c} \sin (\omega t)$, Where $E_{d c}$ represents the time-average potential (the DC bias term), and $\Delta E_{a c}$ represents the amplitude of the AC potential modulation at angular frequency $\omega$. Analogously, under small amplitudes of an AC potential modulation, the modulated optical signal of the redox species adsorbed onto the surface can be described by Equation 3-1.

$$
I=I_{d c}+\Delta I_{a c} \sin (\omega t+\theta)
$$

where $I_{d c}$ represents the time average modulated optical signal. The term $\Delta I_{a c}$ represents the amplitude, and the term $\theta$ represents the phase of the optical signal response originated under the electric potential modulation in the presence of a redox active molecule. Since the baseline modulated optical signal of the EC-SPR device is change with the potential modulation (as described on chapter 1), therefore, to reach accurate results the baseline term will include in the measurements and calculations. The modulated optical response of the baseline signal can be described by Equation 3-2. 
$I_{0}=I_{d c, 0}+\Delta I_{a c, 0} \sin \left(\omega t+\theta_{0}\right)$

Equation 3-2

where $I_{d c, 0}$ represents the time average modulated optical signal. The term $\Delta I_{a c, 0}$ represents the amplitude, and the term $\theta_{0}$ represents the phase of the optical signal response induced by the electric potential modulation in the absence of the redox species. As a result, the modulated optical response to an AC-potential modulation will depend on the difference between the measurement of the baseline and the measurement of the sample and can be described by Equation 3-3.

$$
\Delta I_{S P R}=I_{d c}+\Delta I_{a c} \sin (\omega t+\theta)-I_{d c, 0}-\Delta I_{a c, 0} \sin \left(\omega t+\theta_{0}\right)
$$

The faradaic current associated by redox species adsorbed on an electrode measured optically by SPR technique at a fixed incident of light shows a proportional to the optical signal changes [135], therefore the faradaic current, $I_{a c}^{F}$, can be expressed as in Equation 3-4:

$I_{a c}^{F}=C \times \frac{d}{d t}\left[\Delta I_{S P R}\right]$ Equation 3-4

Where $\mathrm{C}$ is a constant depend on local refractive indices per unit concentration of the oxidized and reduced species on the electrode surface, the mole number of electrons transferred in the reaction, and the Faraday constant. Then by taking the derivative of Equation 3-4 with respect to time, $t$, we have:

$I_{a c}^{F}=C \times \Delta I_{a c} \omega \cos (\omega t+\theta)-\Delta I_{a c, 0} \omega \cos \left(\omega t+\theta_{0}\right)$

Equation 3-5 can be further simplified to:

$$
\begin{aligned}
& I_{a c}^{F}=C \times\left\{\Delta I_{a c} \omega[\cos (\omega t) \cos (\theta)-\sin (\omega t) \sin (\theta)]-\Delta I_{a c, 0} \omega\left[\cos (\omega t) \cos \left(\theta_{0}\right)-\right.\right. \\
& \left.\left.\sin (\omega t) \sin \left(\theta_{0}\right)\right]\right\}
\end{aligned}
$$


$I_{a c}^{F}=C \times \omega\left\{\left(\Delta I_{a c} \cos (\theta)-\Delta I_{a c} \cos \left(\theta_{0}\right)\right) \cos (\omega t)-\left(\Delta I_{a c} \sin (\theta)-\right.\right.$

$\left.\left.\Delta I_{a c} \sin \left(\theta_{0}\right)\right) \sin (\omega t)\right\}$

Equation 3-6

The in-phase and the out-of-phase components of the faradaic current can extract from Equation 3-6 and is given by Equation 3-7 and Equation 3-8, respectively.

$I^{\prime F}{ }_{a c}=C \times \omega\left\{\left(\Delta I_{a c} \sin \left(\theta_{0}\right)-\Delta I_{a c} \sin (\theta)\right)\right\}$

Equation 3-7

$I_{a c}^{\prime \prime F}=C \times \omega\left\{\left(\Delta I_{a c} \cos (\theta)-\Delta I_{a c} \cos \left(\theta_{0}\right)\right)\right\}$

Equation 3-8

The electrochemical impedance response of a three-electrode electrochemical cell with presence of a redox reaction of surface-immobilized redox molecules is modeled with the equivalent circuit shown in Figure 3-1.

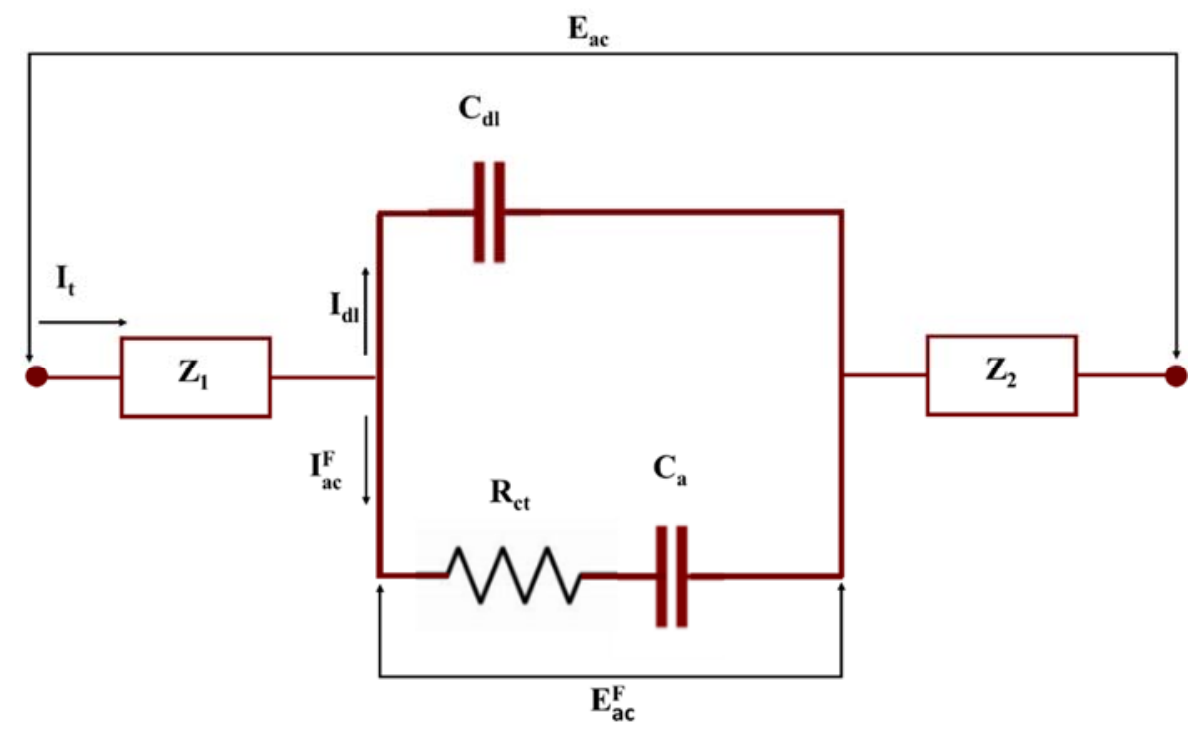

Figure 3-1: Generalized equivalent electrical circuit for the electrochemical flow cell for molecules assemblies.

Where $R_{c t}$ is the charge transfer resistance and $C_{a}$ is the pseudo capacitance. The impedance of the electrochemical flow cell is generalized with $Z_{1}$ and $Z_{2}$, which includes the resistance of the electrolyte solution and other possible impedance sources. $E_{a c}$ is the applied potential modulation across the whole electrochemical flow cell, whereas $E_{a c}^{F}$ is 
the actual potential modulation across the redox reaction. The faradaic admittance, $Y^{F}$, can be represented by[134]:

$Y^{F}=\mathrm{j} \frac{Z_{t o t} I_{a c}^{F} \omega C_{a}}{E_{a c}}=\mathrm{j} \frac{\omega C_{d}}{E_{a c}}\left(I_{a c}^{\prime F}+j I_{a c}^{\prime \prime F}\right)\left(Z_{t o t}^{\prime}+j Z_{t o t}^{\prime \prime}\right)$

Equation 3-9

Then, by expanding Equation 3-9, the real and imaginary components of the faradaic admittance are expressed separately as:

$$
\begin{aligned}
& Y^{\prime F}=-\frac{\omega C_{d}}{E_{a c}}\left[I_{a c}^{\prime F} Z_{t o t}^{\prime \prime}+I_{a c}^{\prime \prime F} Z_{t o t}^{\prime}\right] \\
& Y^{\prime \prime F}=\frac{\omega C_{d}}{E_{a c}}\left[I_{a c}^{\prime F} Z_{t o t}^{\prime}-I_{a c}^{\prime \prime F} Z_{t o t}^{\prime \prime}\right]
\end{aligned}
$$

Where $Z_{\text {tot }}^{\prime}$ and $Z_{\text {tot }}^{\prime \prime}$ are the real and imaginary components of the total electrical impedance across the flow cell, respectively. Using the AC impedance measurements, the total electrical impedance across the flow cell, $Z_{\text {tot }}$, with the real $Z_{\text {tot }}^{\prime}$ and imaginary $Z_{\text {tot }}^{\prime \prime}$ components at each modulation frequency applied, can be measured by a potentiostat. The faradaic current density, $I_{a c}^{F}$, with the real, $I_{a c}^{\prime F}$, and imaginary, $I_{a c}^{\prime F}$, components is optically determined as described in Equation 3-7 and Equation 3-8. Finally, real and imaginary components of the faradaic admittance can be calculated by Equation 3-10 and Equation 3-11.

The faradaic admittance can be expressed theoretically as in Equation 3-12 and the $R_{c t}$ and $C_{a}$ can be expressed by Equation 3-13 and Equation 3-14, respectively.

$$
\begin{aligned}
& \frac{1}{Y^{F}}=R_{c t}+\frac{1}{j \omega C_{a}} \\
& R_{c t}=\frac{Y^{\prime F}}{\left|Y^{F}\right|^{2}} \\
& C_{a}=\frac{\left|Y^{F}\right|^{2}}{\omega Y^{\prime \prime F}}
\end{aligned}
$$


By substituting the expression of $R_{c t}$ and $C_{a}$ into Equation 3-12, the electron-transfer rate constant, $k_{E T}$,can be expressed as in Equation 3-15:

$$
k_{E T}=\frac{1}{2 C_{a} R_{c t}}=\frac{Y^{\prime \prime F}}{2 Y^{\prime F}} \omega
$$

A plot of $y=\omega Y^{\prime \prime F}$ against $x=2 Y^{\prime F}$ calculated over several modulated angular frequencies, $\omega$, can be used to extract the electron-transfer rate, $k_{E T}$, of the redox process by calculating the slope of the linear fitting.

\subsection{Experimental Setup and Procedures}

For an AC potential modulation performed to drive an electrochemical redox process of a monolayer of redox molecules, the same EC-SPR setup used in chapter 2 was improved, as shown in Figure 3-2.

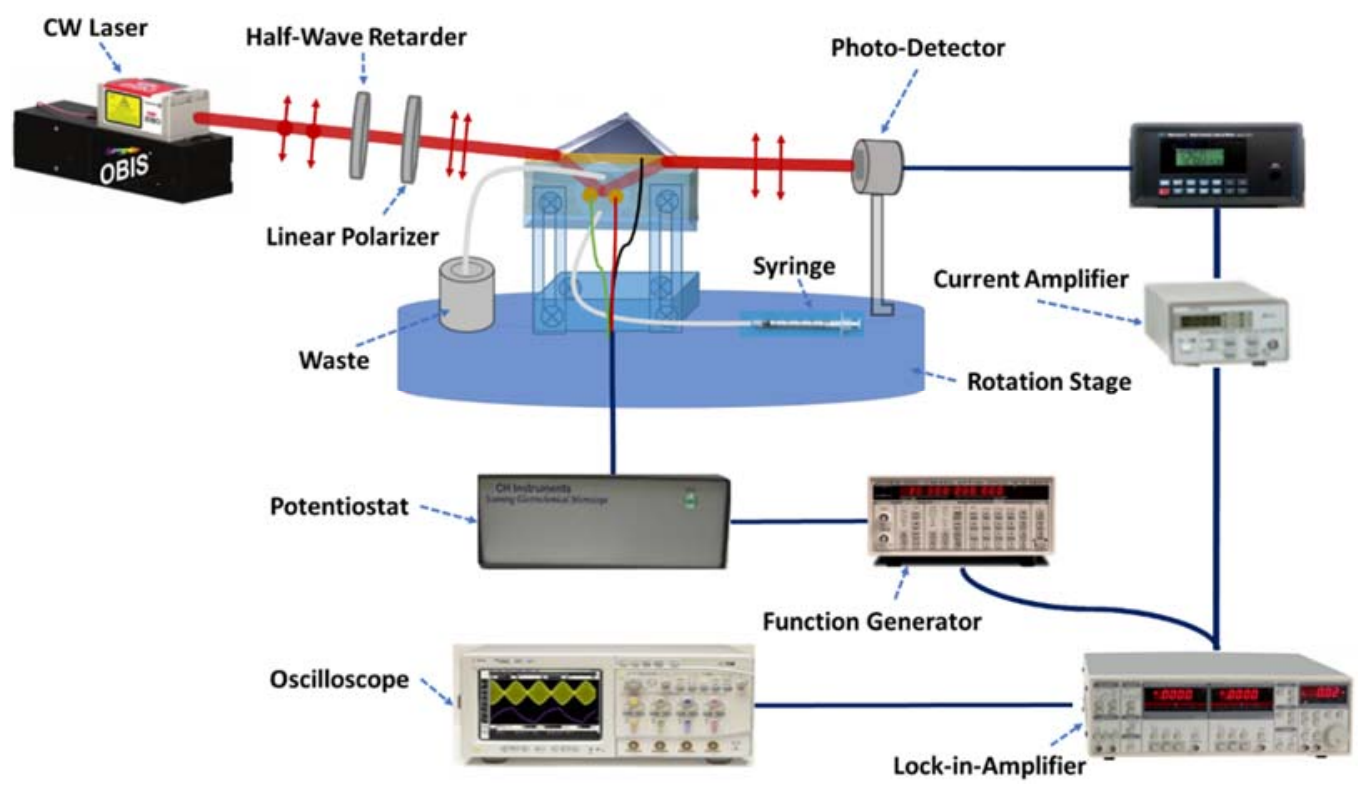

Figure 3-2: Schematic diagram of EC-SPR setup with a potentiostat for electrical control, laser light, polarizer, and half-wave retarder for P-polarized light. A detector, current amplifier, lock-in amplifier, and oscilloscope were used for data collection. 
Three-layer stack of chromium $(5 \mathrm{~nm})$, silver $(35 \mathrm{~nm})$, and gold $(5 \mathrm{~nm})$ thin-films were used as a device interface. The bilayered (silver/gold)-coated glass slide was mounted in a homemade micro-electrochemical flow cell with a three-electrode configuration. The electrochemical flow cell was then mounted on a rotation stage to control the incident angle. The beam of a CW laser (Obis from Coherent, $40 \mathrm{~mW}, \lambda=552 \mathrm{~nm}$ ) was used. A CV scan from $-0.3 \mathrm{~V}$ to $+0.3 \mathrm{~V}$ was used to stabilize the surface before the optical reference signal was recorded. A function generator (DS345, Stanford Research Systems) was connected to the potentiostat to an AC impedance potential modulation to drive the oscillation of the redox proteins across its redox states. The modulated optical signal of the reflected light was monitored at a fixed incident angle by a power meter (Newport, model 1930C) and amplified by a pre-current amplifier. The modulated optical signal was delivered to a lock-in amplifier (SR830 DSP, Stanford Research Systems), where the magnitude and the phase of the analytical signal (for both the reference signal and the signal after immobilizing the cytochrome c protein onto the EC-SPR platform) were collected. Finally, the analytical signal was recorded by an oscilloscope (Agilent, DSO8104A Infiniium).

\subsection{Functionalization Protocols of the Platform with Different Layers}

The immobilization strategies of molecular assemblies at electrode surfaces are crucial for stabilizing biomolecules on the surface, reducing the conformational changes and eliminate the non-specific adsorption. In this work, two types of SAM's deposited on the EC-SPR platform were used in order to attach the interest biological materials: I) assemblies of carboxylic acid terminated SAM's of MPA. The MPA modifier provides a negatively charged surface and was used to attach the cytochrome c protein onto the 
surface. II) Cysteamine (2-aminoethanethiol) (CYS) (purchased from Sigma-Aldrich (St. Louis,Missouri)) which provide a positively charged surface. The CYS molecules are attached strongly to the gold surfaces due to the high affinity between their thiol groups and the gold surface, whereas the amine groups of the CYS molecules are used to capture the antibodies through electrostatic interactions [136].

\subsubsection{Immobilization of Cytochrome c protein onto MPA-modified EC-SPR Platform}

For assemblies of cytochrome c protein onto the MPA-modified EC-SPR platform, two different immobilization strategies were employed: I) electrostatic binding between the positive charges of cytochrome c protein and the negative charges of the MPA film, as described in chapter 1. Briefly, the MPA-coated EC-SPR platform was mounted on an electrochemical flow cell. A PBS buffer solution was injected into the flow cell. A CV potential modulation from $-0.3 \mathrm{~V}$ to $0.3 \mathrm{~V}$ at a scan rate of $0.02 \mathrm{~V} / \mathrm{s}$ was used to stabilize the MPA- modified EC-SPR platform. After the stabilization process, a $50 \mu \mathrm{L}$ solution of cytochrome c protein at a concentration of $500 \mathrm{nM}$ was incubated in the flow cell for 30 minutes. Then, the flow cell was thoroughly rinsed with a PBS buffer solution to remove unbound cytochrome c protein from the surface. II) Covalent binding between the cytochrome c protein and the activated terminal carboxylic groups of the MPA film. A mixed of $2 \mathrm{mM}$ of 1-ethyl-3-(3-dimethylaminopropyl) carbodiimide (EDC) and $5 \mathrm{mM}$ of N hydroxy succinimide (NHS) (purchased from Sigma-Aldrich (St. Louis,Missouri)) in PBS buffer solution was incubated in the flow cell for 1 hour to convert the terminal carboxylic groups of the MPA film to active NHS esters. Subsequently, a covalent bond between cytochrome c protein and the active NHS ester was formed by incubating a solution of $500 \mathrm{nM}$ cytochrome c protein in the flow cell for 30 minutes. Then, the flow 
cell was rinsed with a PBS buffer solution to remove unbound cytochrome c protein from the surface. Finally, a CV potential modulation from $-0.3 \mathrm{~V}$ to $0.3 \mathrm{~V}$ at a scan rate of 0.02 V/s was used to stabilize the adsorbed cytochrome c protein on the MPA-coated EC-SPR platform.

\subsubsection{Immobilization of Cytochrome C Antibody (Cyt c Ab) onto CYS- modified EC-}

\section{SPR platform}

SAM's based on a CYS molecules was used to immobilize the Cyt c Ab onto the ECSPR platform. For this purpose, the cleaned platform was immersed in freshly prepared 10 mM DI water solution of CYS at room temperature for 12 hours[137]. The CYS- modified EC-SPR platform was then washed with DI water to remove the weak and unbound thiols and then gently dried under $\mathrm{N}_{2}$ gas. After mounting the CYS- modified EC-SPR platform on an electrochemical flow cell, a $0.5 \mu \mathrm{g} / \mathrm{ml}$ of Cyt c Ab (A-8) mouse monoclonal IgG2b (purchased from Santa Cruz Biotechnologies, Inc (Dallas, Texas)) in the PBS buffer solution was incubated in the flow cell for 2 hours. Finally, the adsorbed cytochrome c antibodies onto CYS-modified EC-SPR platform were stabilized under CV potential modulation from $-0.3 \mathrm{~V}$ to $0.3 \mathrm{~V}$ at a scan rate of $0.02 \mathrm{~V} / \mathrm{s}$.

\subsubsection{Bovine Serum Albumin (BSA)}

Due to the stability of BSA protein and it ability to attach to both positively and negatively charged surfaces, it is commonly used for attachment studies and kinetic measurements. In the biosensor field, the BSA mainly used to reduce the background and the non-specific binding on a surface of interest[138, 139]. In this study, the effect of adding the BSA onto the Cyt $\mathrm{c}$ Ab/CYS-modified EC-SPR platform on the electrontransfer rate was examined. To do so, and after CYS-modified EC-SPR platform was 
stabilized, a $50 \mu \mathrm{l}$ of $1 \%$ BSA solution (purchased from Sigma-Aldrich (St. Louis, Missouri)) was incubated in the flow cell for 30 minutes. Then, the flow cell was rinsed with a PBS buffer solution and stabilized under CV potential modulation.

\subsection{Results and Discussion}

In order to investigate the impact of different functionalized layers and immobilization strategies on the electron-transfer rate of redox proteins, cytochrome c protein has been used as a model system. Different immobilization strategies were examined. I) Direct adsorption, where the cytochrome c protein are immobilized onto the surface through weak van der Waals forces and hydrophobic interactions[126, 140, 141], II) electrostatic adsorption, where the cytochrome c protein are absorbed electrostatically to SAM's composed of carboxylic acid terminated thiols[142], III) covalent adsorption, where the cytochrome c protein are attached covalently onto activated SAM's [125, 143], and IV) Affinity adsorption[124], where the cytochrome c protein are captured by Cyt c $\mathrm{Ab}$ that attached to the surface by SAM's based on the amino groups using CYS. The redox formal potentials of cytochrome c protein at these functionalized layers are determined electrochemically and optically. The electron-transfer kinetics of cytochrome c protein in these four systems are investigated.

\subsubsection{Electroactive Surface Coverage Measurements}

A cyclic voltammetry $(\mathrm{CV})$ technique was used to investigate the electroactive surface coverage of the cytochrome c protein binding onto the functionalized layers noted earlier. The same setup as shown in Figure 3-2, was used. CV potential scans at several different potential range and scan rates of $0.02 \mathrm{~V} / \mathrm{s}$ were performed. The electrical current 
response under the CV scans was collected. Figure 3-3 shows the electrical current response under the $\mathrm{CV}$ scans obtained for cytochrome c protein absorbed onto different functionalized-EC-SPR platform.

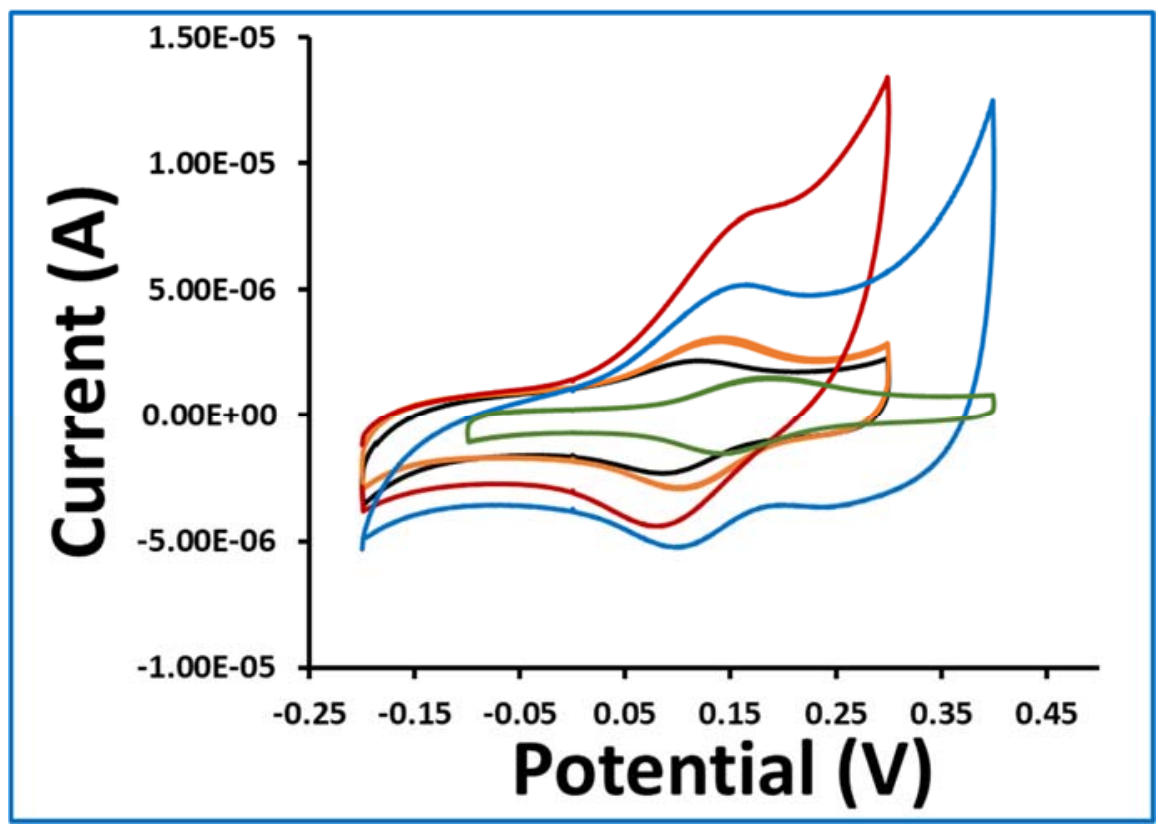

Figure 3-3: The electrical current response under the $\mathrm{CV}$ scan for cytochrome $\mathrm{c}$ protein in $10 \mathrm{mM}$ PBS buffer solution at scan rate of $0.02 \mathrm{~V} / \mathrm{s}$. Black curve represents the direct adsorption, orange curve represents the electrostatic adsorption, blue cure represents the covalent adsorption, red curve represents the affinity adsorption, and green curve represents the affinity adsorption with the presence of the BSA.

The electrical current curves under the CV scans show obvious signs of current peaks associated with redox reactions of the immobilized cytochrome $\mathrm{c}$ protein redox reactions. Using these data, the electroactive surface coverage of cytochrome c protein adsorbed onto the modified platform at each functionalized layer was estimated. A Gaussian fitting of the 
reduction peaks for each functionalized layers was determined, Figure 3-4.

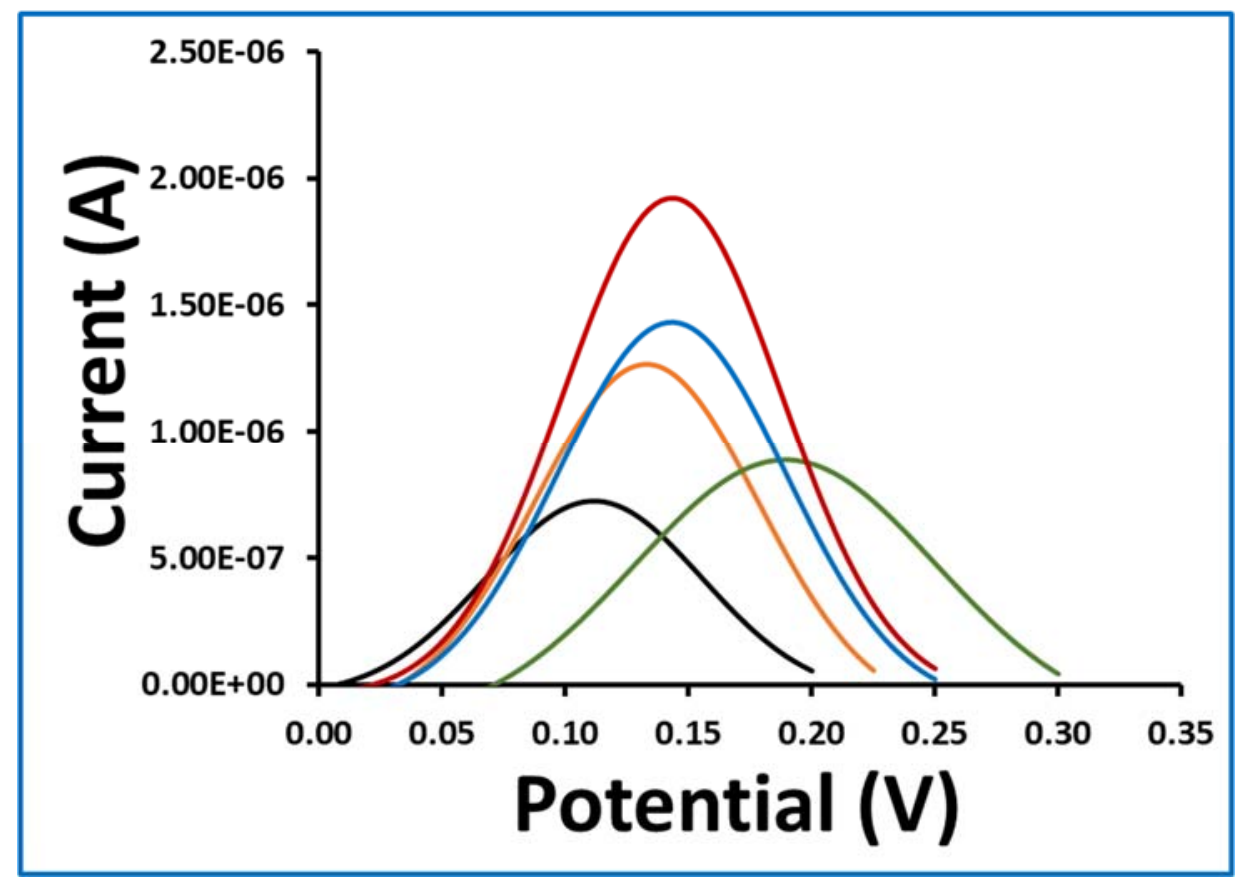

Figure 3-4: Gaussian fitting of the reduction peaks for cytochrome c protein assemblies. Black curve represents the direct adsorption, orange curve represents the electrostatic adsorption, blue cure represents the covalent adsorption, red curve represents the affinity adsorption, and green curve represents the affinity adsorption with the presence of the BSA. The integration of each reduction peak was measured. Equation 2-2 was used for estimating the electroactive surface coverage of cytochrome $\mathrm{c}$ protein adsorbed onto the modified platform at each functionalized layer. The electroactive surface coverage of cytochrome c protein adsorbed at each functionalized layer are shown in Table 3-1.

Table 3-1:The electroactive surface coverage of cytochrome c protein adsorbed at each functionalized layer by a cyclic voltammetry (CV) technique. 


\begin{tabular}{|l|c|}
\hline EC-SPR functionalized surface & Electroactive surface coverage $\left(\mathrm{pm} / \mathrm{cm}^{2}\right)$ \\
\hline EC-SPR platform & 2.67 \\
\hline MPA/EC-SPR platform & 4.73 \\
\hline (ED-NHS)/MPA/EC-SPR platform & 5.59 \\
& 7.53 \\
\hline Cyt c Ab/CYS/EC-SPR platform & 4.13 \\
\hline BSA/Ab/CYS/EC-SPR platform & \\
\hline
\end{tabular}

The results in Table 3-1, show different electroactive surface coverage, ranging from 2.67 to $7.53 \mathrm{pm} / \mathrm{cm}^{2}$. The direct adsorption of cytochrome c protein onto the EC-SPR platform yields a minimum electroactive surface coverage. This indicates that the direct adsorption leads to a random orientation of the immobilized cytochrome $\mathrm{c}$ protein which results in poor electrochemical behavior due to denaturation and shielding of active binding sites of the proteins[144]. II) The increase in the electroactive surface coverage with the electrostatic and covalent interactions indicate that these immobilization strategies protect the proteins from the denaturation and can improve their orientation[113]. III) The maximum electroactive surface coverage performs with the high-affinity binding between the cytochrome c protein and the Cyt $\mathrm{c} \mathrm{Ab}$. This indicates that the antibody can enhance and improve the cytochrome c protein's orientation and electro-activity [113, 145]. IIII) After adding the BSA layer, the electroactive surface coverage was reduced, this could be due to the blocking of some efficient electron-tunneling pathways 


\subsubsection{Redox Formal Potential Measurements}

In this section, a cyclic voltammetry $(\mathrm{CV})$ technique and an $\mathrm{AC}$ voltammetric modulation technique were used for determining the formal potential of the cytochrome c protein binding onto the functionalized layers noted earlier.

\subsubsection{Cyclic Voltammetry (CV) Technique}

The electrical current response under the CV scans obtained for cytochrome c protein adsorbed on different functionalized-EC-SPR platform, Figure 3-3, was used to measure the formal potential. The formal potential was determined as the average of the reduction and oxidation peaks. The results are listed in Table 3-2.

\subsubsection{AC Voltammetric Modulation Technique}

In order to confirm the results in section 3.5.2.1, an $\mathrm{AC}$ voltammetric modulation technique was used for investigating the redox formal potentials of immobilized cytochrome c protein redox reactions at the different functionalizing layers. Sinusoidal potential modulation with $20 \mathrm{mV}$ amplitude and a modulation frequency was employed at several different DC bias potentials. SPR optical signal, $\triangle I_{S P R}$, at each DC bias potential for the immobilized cytochrome c protein redox reactions at different functionalizing layers were obtained by the lock-in-amplifier. The SPR optical signal, $\triangle I_{S P R}$, was determined by subtracting the baseline SPR optical signal, which was taken after stabilizing the modified EC-SPR surface (no redox species) from the SPR optical signal collected after the incubation (and rinsing) of the cytochrome c protein. Figure 3-4 shows the SPR optical signal, $\triangle I_{S P R}$, against the DC bias potential for the immobilized cytochrome c protein redox reactions at different functionalizing layers. 
(a)

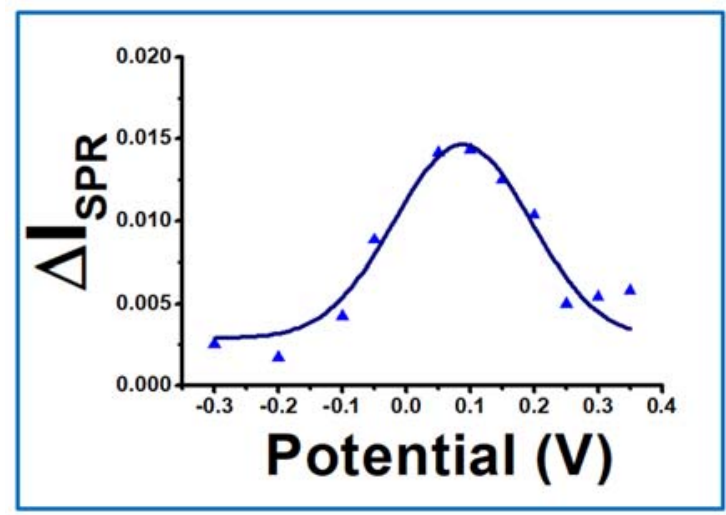

(c)

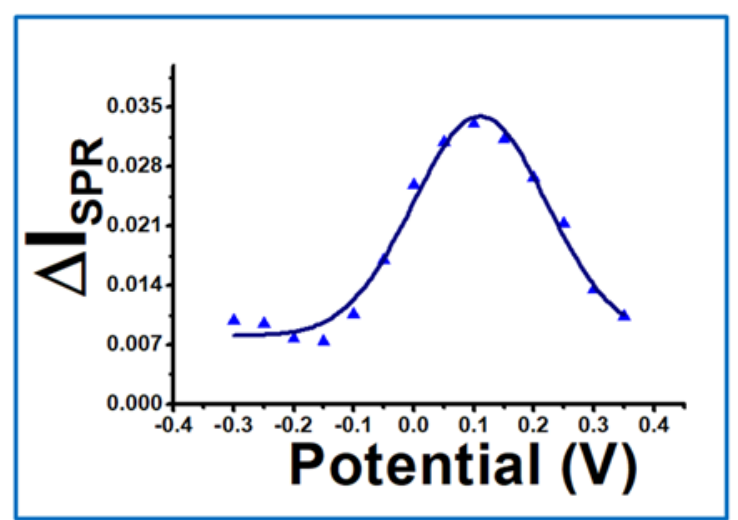

(b)

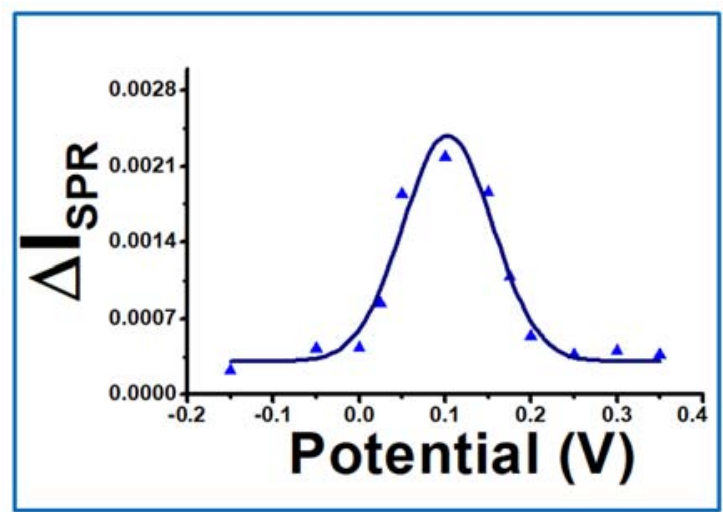

(d)

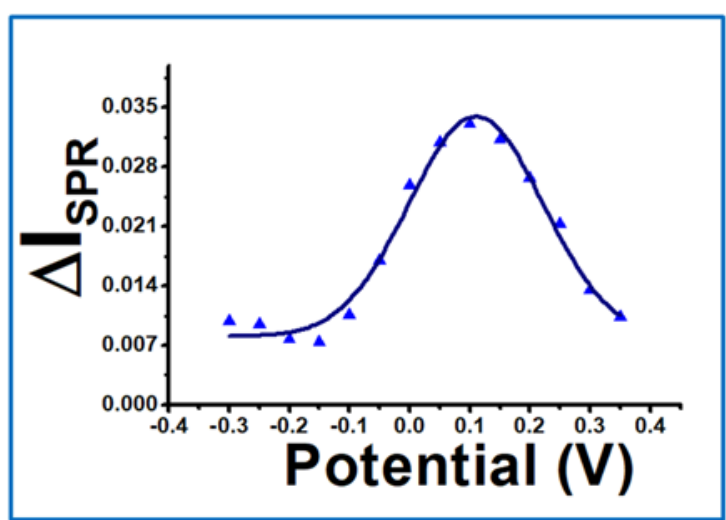

(e) 


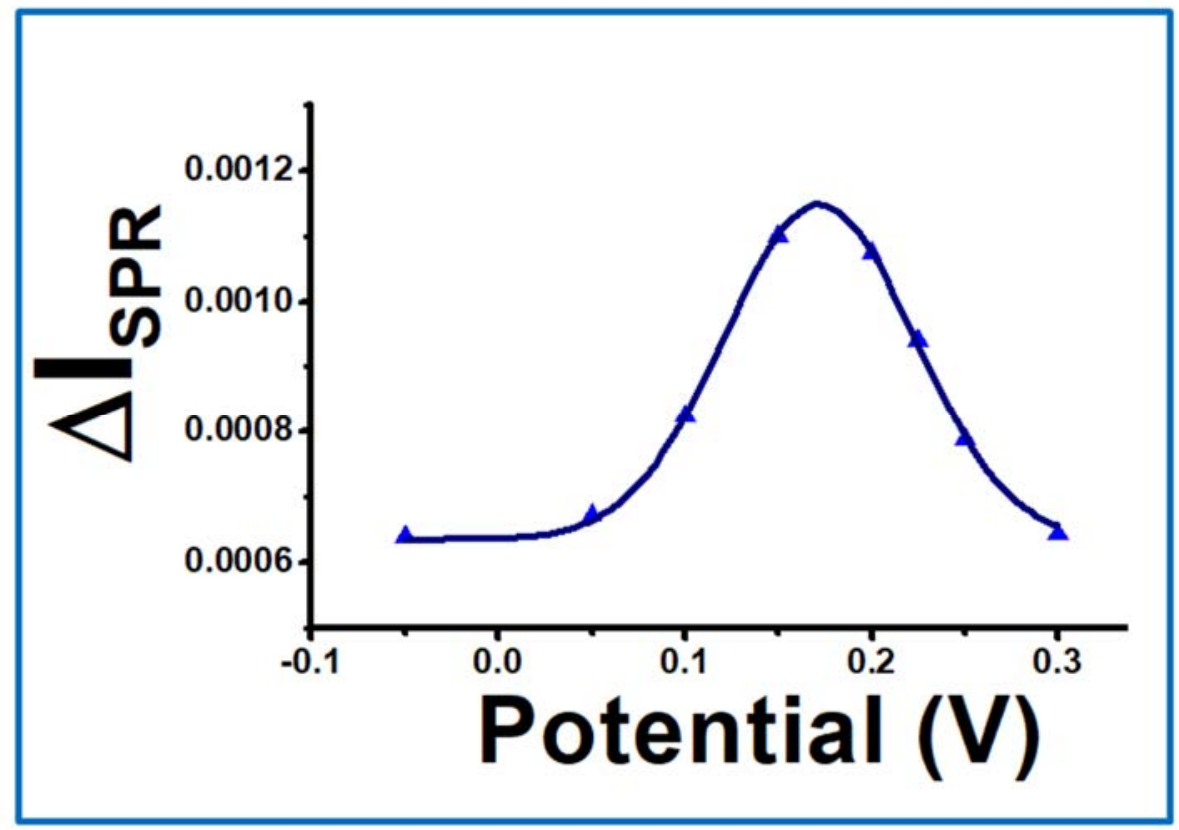

Figure 3-5: SPR optical signal, $\triangle I_{S P R}$, versus the DC bias potential ranging from $(-0.3 \mathrm{~V}$ to $0.35 \mathrm{~V}$ ) measured under a sinusoidal potential modulation with an amplitude modulation of $20 \mathrm{mV}$.

For each immobilization strategy, the plot of the optical signal, $\triangle I_{S P R}$, against the DC bias potential displays a clear peak, which confirms the redox activity of the cytochrome c protein. A Gaussian fit was used to determine the redox formal potential. The redox formal potential under the $\mathrm{CV}$ scan and the $\mathrm{AC}$ impedance potential modulation are compared in Table 3-2.

Table 3-2: The electrical and optical measurements of the redox formal potentials for cytochrome c protein redox reactions at the different functionalized layers on the EC-SPR platform. 


\begin{tabular}{|l|c|c|}
\hline EC-SPR functionalized surface & $\begin{array}{c}\text { Formal potential (V) } \\
\text { (electrical data) }\end{array}$ & $\begin{array}{c}\text { Formal potential (V) } \\
\text { (optical data) }\end{array}$ \\
\hline EC-SPR platform & 0.098 & 0.094 \\
\hline MPA/EC-SPR platform & 0.117 & 0.110 \\
\hline (ED-NHS)/MPA/EC-SPR platform & 0.119 & 0.114 \\
\hline Cyt c Ab/CYS/EC-SPR platform & 0.121 & 0.117 \\
\hline BSA/Ab/CYS/EC-SPR platform & 0.164 & 0.169 \\
\hline
\end{tabular}

Based on Table 3-2, the following observations can be concluded: First, the redox formal potentials of the cytochrome c protein measured optically at different functionalized ECSPR platform are in good agreement with that measured electrically at the same conditions. Second, the formal potential of the adsorbed cytochrome c on the EC-SPR platform strongly depends on the immobilization strategy. Third, a negative shift in redox formal potential of cytochrome c protein directly adsorbed onto the EC-SPR platform $(0.098 \mathrm{~V})$ compared to that of cytochrome c protein electrostatically adsorbed onto the MPAmodified EC-SPR platform $(0.117 \mathrm{~V})$. This indicates that the interaction of cytochrome c protein directly with the electrode surface causes denaturation and changes in the conformation of the native proteins, providing rise to the negative shift of formal redox potential $[13,35,36]$. Fourth, the cytochrome c protein covalently attached to activated terminal carboxylic groups of MPA $(0.117 \mathrm{~V})$ exhibits almost the same formal potential as cytochrome c protein electrostatically adsorbed onto the MPA-modified EC-SPR platform $(0.119 \mathrm{~V})$. This indicates that the MPA-modified the electrode surface can protect the protein from the denaturation and keep it in a native state. Fifth, the specific affinity between the cytochrome c protein and the Cyt $\mathrm{c} \mathrm{Ab}$ causes a positive shift in the redox 
formal potential to $0.121 \mathrm{~V}$ and to $0.164 \mathrm{~V}$ with the presence of the BSA. This formal potential shift could be attributed to presence of the antibody and the BSA on the surface[146].

\subsubsection{Electron-Transfer Rate Measurements}

The electron-transfer rate between cytochrome c protein and different electrode assemblies was measured using the OIS technique based on the EC-SPR platform. A sinusoidal potential modulation with $20 \mathrm{mV}$ amplitude was applied on the surface at the formal potential, and several frequencies ranging from 10 to $500 \mathrm{~Hz}$ were measured. The SPR optical signal, $\triangle I_{S P R}$, was determined by subtracting the baseline SPR optical signal, which was taken with PBS buffer solution (no redox species) in the flow cell from the SPR optical signal collected after the incubation (and rinsing) of the cytochrome c protein in PBS buffer solution. The faradaic current density $I_{a c}^{F}$, with the real ${I^{\prime}}_{a c}^{F}$ and imaginary $I^{\prime \prime}{ }_{a c}^{F}$ components, was optically determined using the in-phase and out-of-phase components of the optical signal as described in Equation 3-7 and Equation 3-8. The real $Z_{\text {tot }}^{\prime}$ and imaginary $Z_{t o t}^{\prime \prime}$ components of the impedance for the immobilized cytochrome c protein on the surface were measured using the potentiostat. The faradaic admittance was determined using Equation 3-10 and Equation 3-11. Finally, a plot of $y=\omega Y^{\prime \prime F}$ against $x=2 Y^{\prime F}$ calculated over several modulated angular frequencies was used to measure the electrontransfer rate.

\subsubsection{Cytochrome c protein Directly Immobilized onto the EC-SPR Platform}

The electron-transfer properties of cytochrome c protein directly immobilized onto the EC-SPR surface was investigated. Three identical samples were used for three different experiments carried out at the same conditions. The redox formal potential was determined 
optically in section 3.5.3.1 to be $0.094 \mathrm{~V}$. An AC potential modulation at fixed DC bias potential $(0.094 \mathrm{~V})$ was applied and several frequencies ranging from $10 \mathrm{~Hz}$ to $500 \mathrm{~Hz}$ were measured. The SPR optical signals, $\triangle I S P R$, versus the frequency, $\omega$, of the sinusoidal potential modulation for the three samples are shown in Figure 3-6.

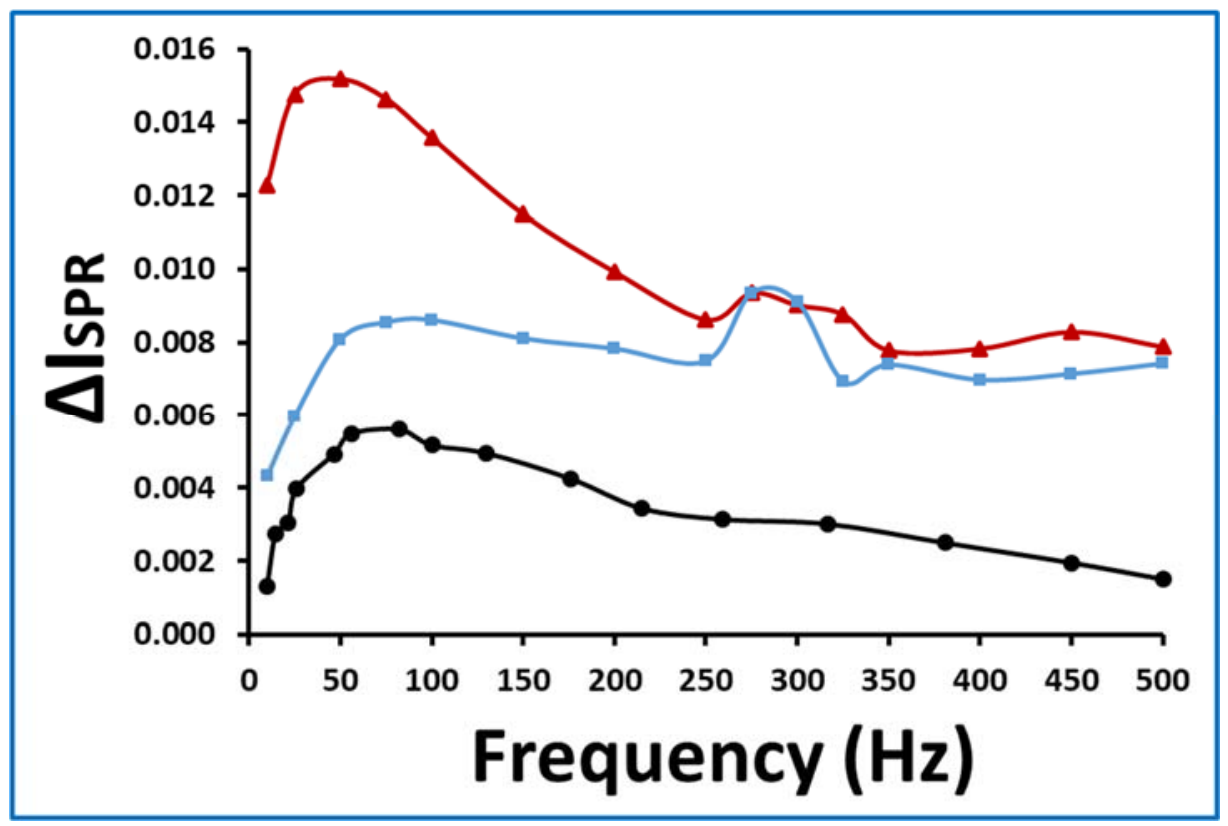

Figure 3-6: SPR optical signal, $\triangle I_{S P R}$, measured under $20 \mathrm{mV}$ AC amplitude of impedance potential modulations at the $\mathrm{DC}$ bias potential of $0.015 \mathrm{~V}$ is plotted against angular modulation frequency for three different experiments at the same conditions.

The three curves in Figure 3-6 represent the SPR optical signal, $\triangle I_{S P R}$, against the frequency, $\omega$, for the three experiments that were carried out at the same conditions. A broad peak centered at about $50 \mathrm{~Hz}$ corresponding to the highest electron-transfer rate of the cytochrome c protein redox process was determined. Since the electron-transfer rate through the cytochrome $\mathrm{c}$ protein is a function of the position of the heme iron with respect to the electrode surface, the broadness of these peaks indicate a random degree of orientation of the immobilized cytochrome c protein. Next, in order to measure the 
electron-transfer rate, the real and imaginary components of the faradaic current densities were extracted from the optical data presented in Figure 3-5 with frequencies ranging from $50 \mathrm{~Hz}$ to $500 \mathrm{~Hz}$. On the other hand, a conventional EIS technique was used to determine the real and imaginary components of the total electrical impedance, Figure 3-7.

(a)

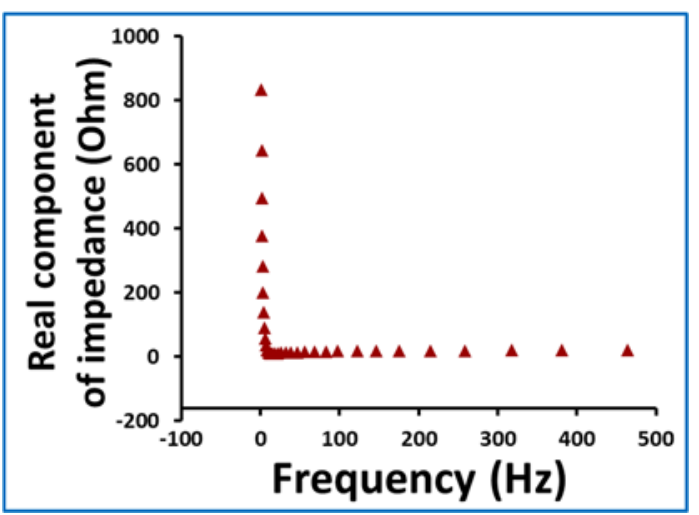

(b)

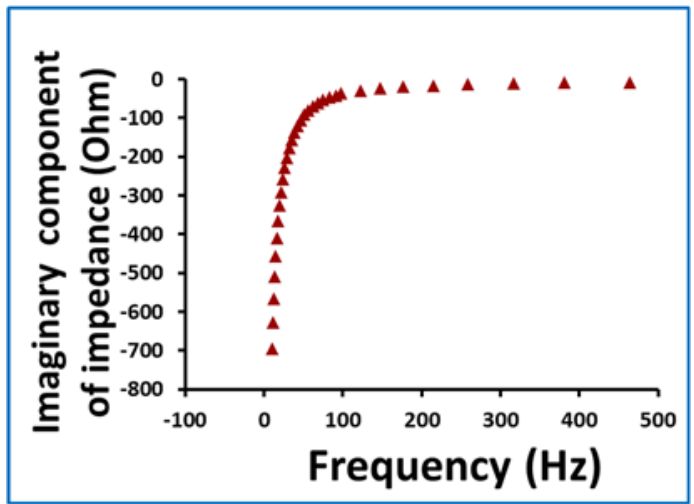

Figure 3-7: (a) The real components of the total electrical impedance results of the electrochemical flow cell for cytochrome c protein directly absorbed onto EC-SPR platform, where (b) represents the imaginary components.

Based on the optical and the total electrical impedance data, the real and imaginary components of the faradaic admittance were calculated. Finally, a plot of $y=\omega Y^{\prime \prime F}(\omega)$ 
against $x=2 Y^{\prime F}(\omega)$ calculated over several modulated angular frequencies, $\omega$, were used to extract the electron-transfer rate of the faradic process, Figure 3-8.

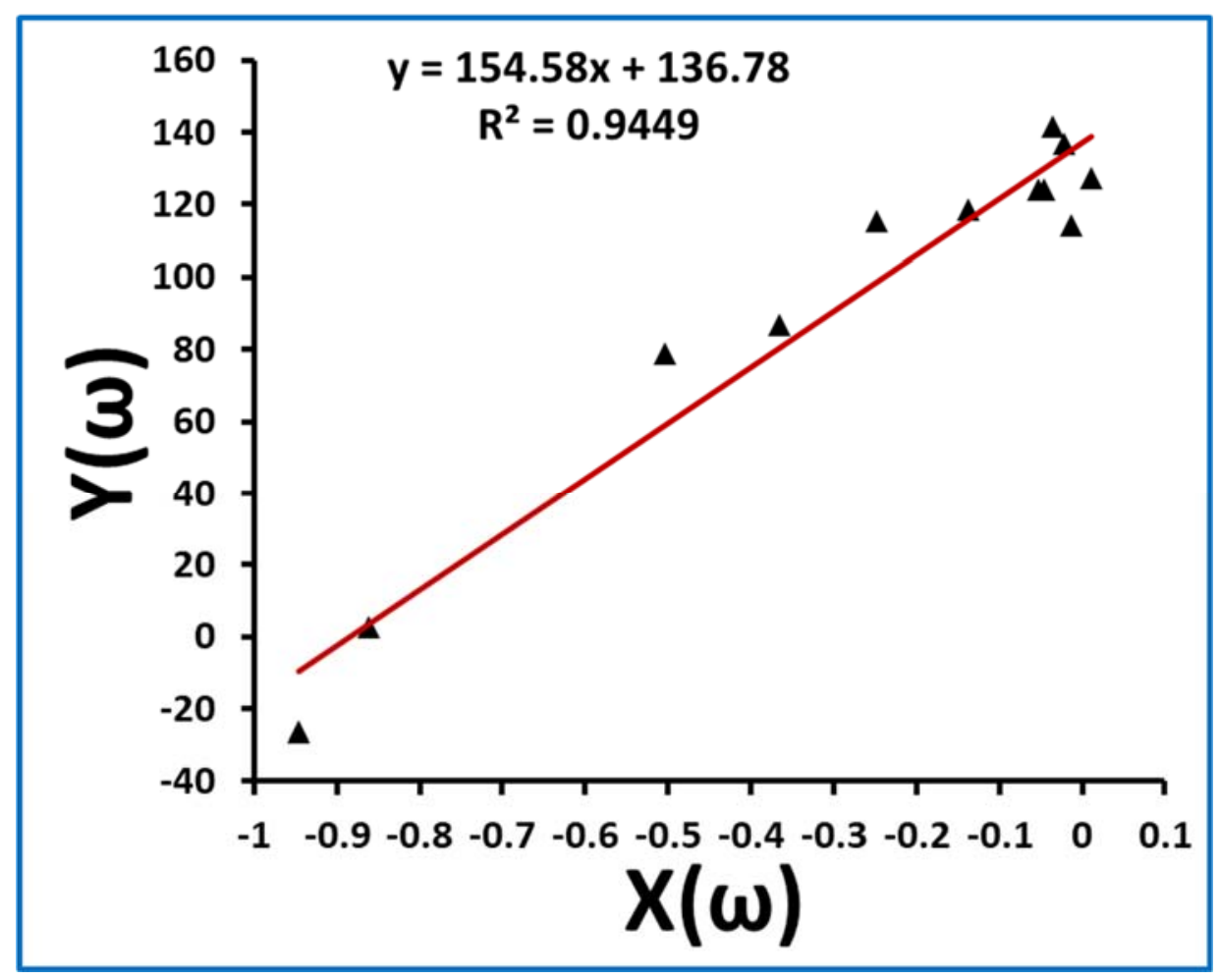

Figure 3-8: Electron-transfer rate calculation. The electron-transfer rate is represented by the slope of the linear fit.

The slope of the linear fitting provides the electron-transfer rate between the heme of cytochrome c protein and the EC-SPR surface. The same procedures were used to measure the electron-transfer rate using the other two samples (Appendix B-1). The average of the electron-transfer rate for three experiments was $(1.7 \pm 0.1) \times 10^{2} s^{-1}$. 


\subsubsection{Cytochrome c protein Absorbed onto MPA-modified EC-SPR Platform}

The electron-transfer properties for the cytochrome c protein that were immobilized onto the MPA-modified EC-SPR platform were investigated. Two different immobilization strategies (electrostatic and covalent) of absorbed cytochrome c protein onto the MPA-modified EC-SPR platform were examined. The formal potential was measured electrically in section 3.5.3.1 to be $0.0110 \mathrm{~V}$ for electrostatic adsorption and $0.0114 \mathrm{~V}$ for covalent adsorption. Three identical samples were used for three different experiments carried out at the same conditions. Figure 3-9 shows the SPR optical signal, $\triangle I_{S P R}$, versus the frequencies of the sinusoidal potential modulation for (a) electrostatic immobilization, and (b) covalent immobilization.

(a)

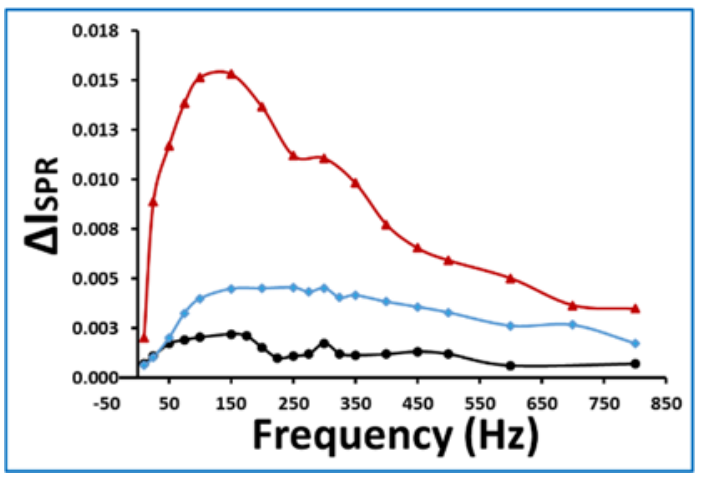

(b)

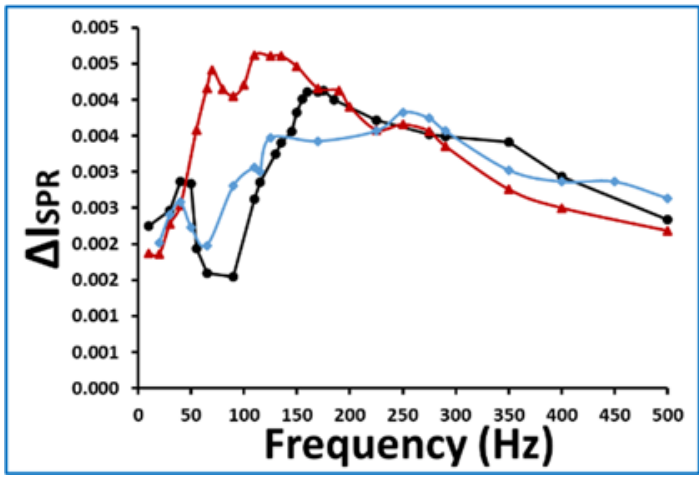

Figure 3-9: SPR optical signal, $\triangle I_{S P R}$, measured under $20 \mathrm{mV}$ AC amplitude of impedance potential modulations at the formal potential is plotted against angular modulation frequency for (a) electrostatic immobilization, and (b) covalent immobilization.

Figure 3-6 (a) shows broad peaks centered at about $150 \mathrm{~Hz}$. The broadness of these peaks indicates that the electrostatic adsorption of cytochrome c protein onto the MPA-modified EC-SPR platform leads to a broad distribution of cytochrome c protein's orientation on the 
surface. Figure 3-6 (b) shows two peaks centered at about 50 and $150 \mathrm{~Hz}$, which indicates the existence of more types of interactions between the surface and cytochrome c protein. As mentioned in the previous section, the peak at $50 \mathrm{~Hz}$ is corresponding to the electron transfer of the cytochrome $\mathrm{c}$ protein redox process that directly absorbed to the EC-SPR platform. Therefore, the peak centered at $150 \mathrm{~Hz}$ should be related to the electron transfer of the cytochrome $\mathrm{c}$ protein redox process that adsorbed onto the MPA-modified platform. The existence of these two peaks indicates that the adsorption of cytochrome $\mathrm{c}$ protein onto the activated MPA-modified EC-SPR platform shows a higher degree of orientation (narrow distribution of orientations) compared to the electrostatic adsorption. Next, in order to determine the electron transfer-rate of the cytochrome c protein at these two immobilization strategies, the optical data shown in Figure 3-9 were used. The real and imaginary components of the faradaic current densities were determined. For the electrostatic immobilization, the frequencies ranging from $10 \mathrm{~Hz}$ to $250 \mathrm{~Hz}$ were used, whereas, for the covalent immobilization, the frequencies were ranging from $75 \mathrm{~Hz}$ to 250 Hz. Meanwhile, the real and imaginary components of the total electrical impedance were extracted using a conventional EIS technique. Figure 3-10 (a) and (b) display the real and imaginary components of the impedance for cytochrome c protein electrostatically adsorbed onto the MPA-modified EC-SPR platform, while (c) and (d) for covalently absorbed onto the activated MPA-modified EC-SPR platform. 


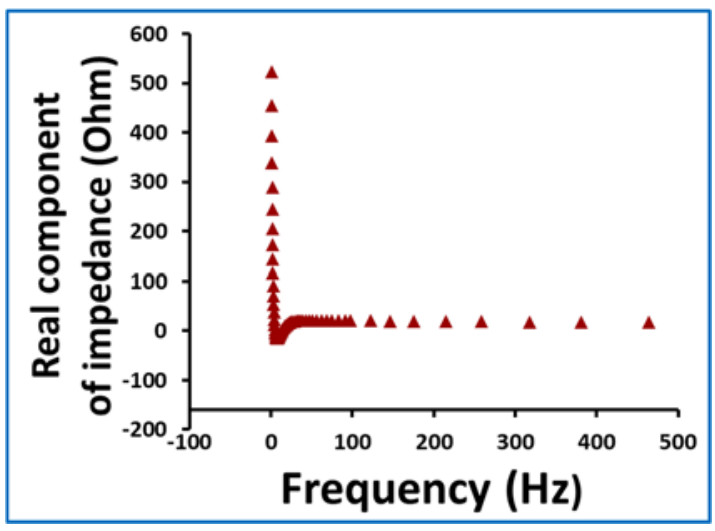

(c)

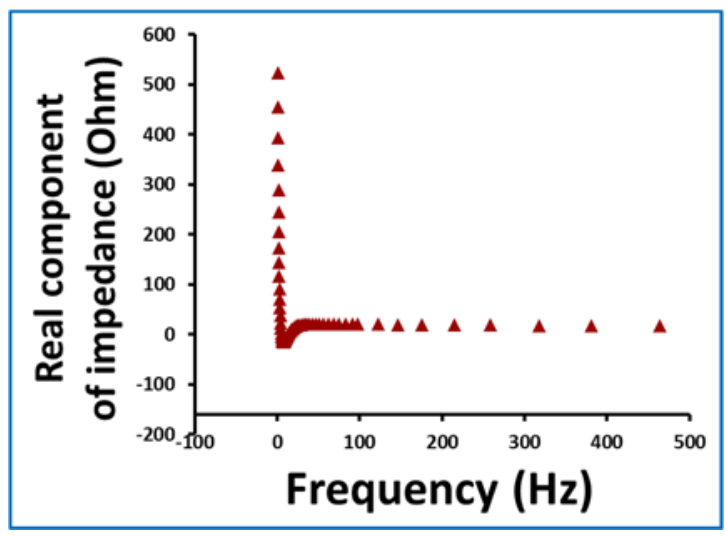

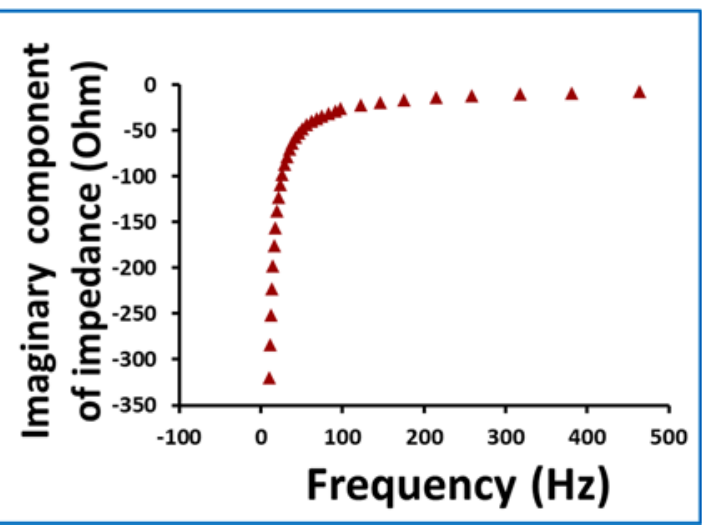

(d)

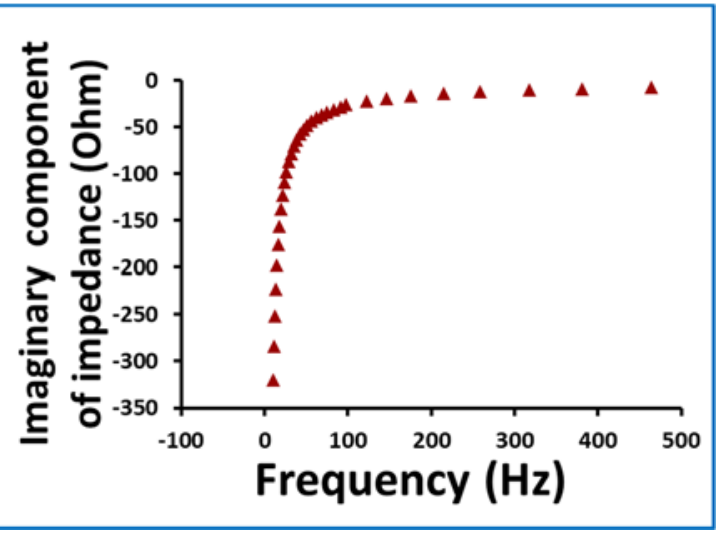

Figure 3-10: Electrical impedance results of the electrochemical flow cell, (a) represents the real component of the total electrical impedance for electrostatic adsorption, whereas (b) the imaginary component. (c) represents the real component of the total electrical impedance for covalent adsorption, whereas (d) the imaginary component.

Next, the optical and the total electrical impedance data were used to measure $y=$ $\omega Y^{\prime \prime F}(\omega)$ and $x=2 Y^{\prime F}(\omega)$. Figure 3-11 displayed a plot of y against $\mathrm{x}$ calculated over several modulated angular frequencies. 

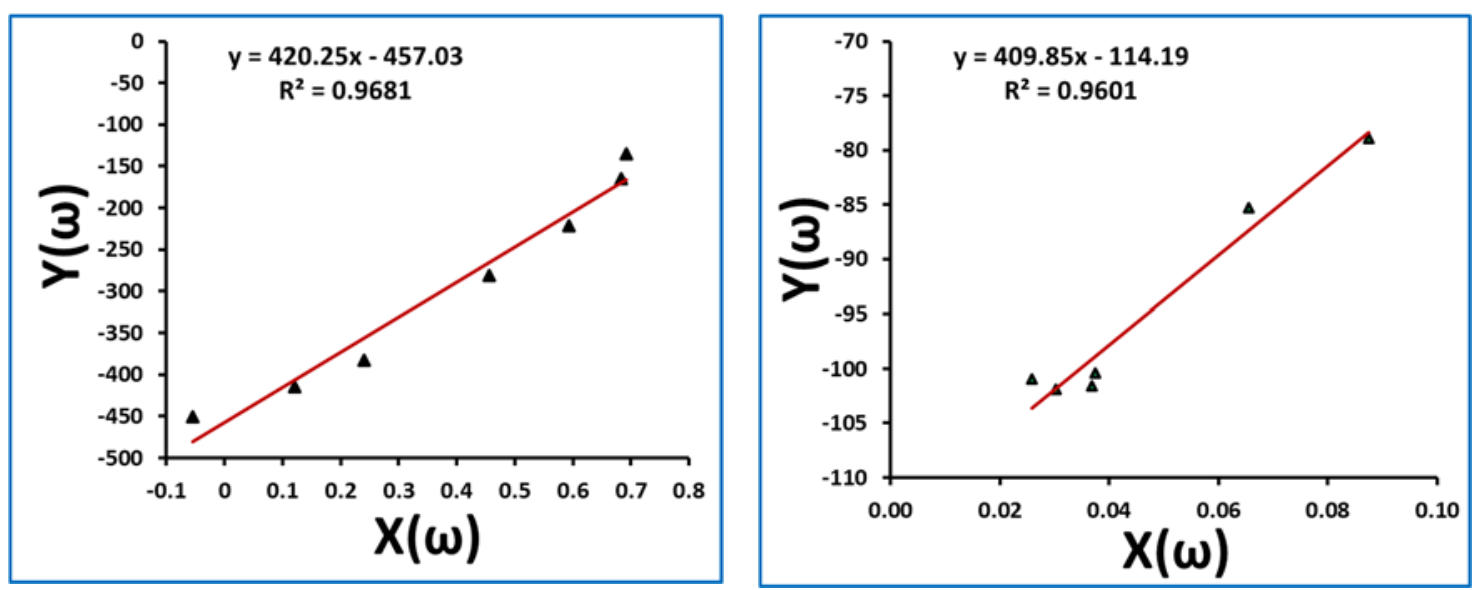

Figure 3-11: Electron-transfer rate calculation. The electron-transfer rate is represented by the slope of the linear fit, (a) for electrostatic adsorption, whereas (b) for covalent adsorption.

Finally, the electron-transfer kinetics of cytochrome c protein immobilized onto the MPAmodified EC-SPR platform were extracted from the slope of the linear fitting. The average of electron-transfer rate of the three experiments was $(4.1 \pm 0.6) \times 10^{2} \mathrm{~s}^{-1}$ for the electrostatic adsorption and $(4.2 \pm 0.5) \times 10^{2} \mathrm{~s}^{-1}$ for the covalent adsorption.

\subsubsection{Cytochrome c protein Absorbed onto Cyt c Ab /CYS-Modified EC-SPR Platform}

The methodology of OIS, based on an EC-SPR, was used to measure the electron transfer properties of the cytochrome c protein that were immobilized onto Cyt c Ab/CYSmodified EC-SPR platform. The same methodology was used to examine the effect of present a BSA layer on the electron-transfer rate. The redox formal potential of the cytochrome c protein attached to the Cyt c Ab/CYS-modified EC-SPR platform was determined in section 3.5.2.3 to be $0.117 \mathrm{~V}$, and $0.169 \mathrm{~V}$ with presence of BSA layer. The same procedure described in the last section was carried out. Figure 3-12 shows the SPR optical signal, $\triangle I_{S P R}$, versus the frequencies of the sinusoidal potential modulation for the 
cytochrome c protein captured by Cyt c Ab. Three different experiments at the same conditions are displayed.

(a)

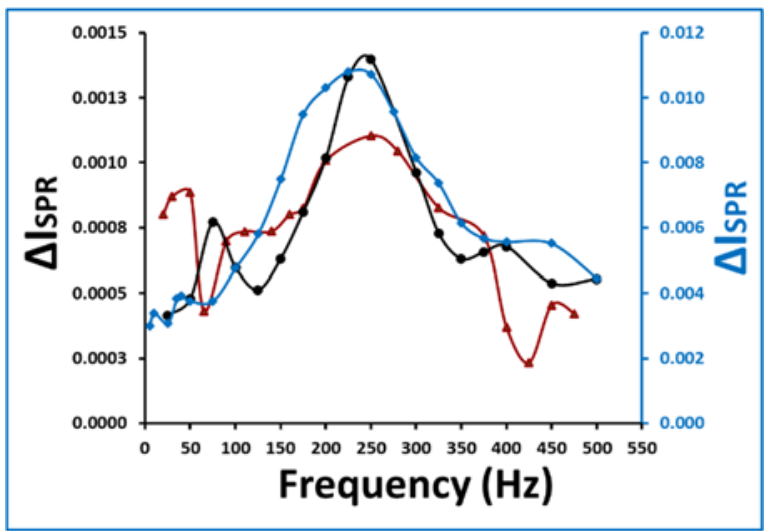

(b)

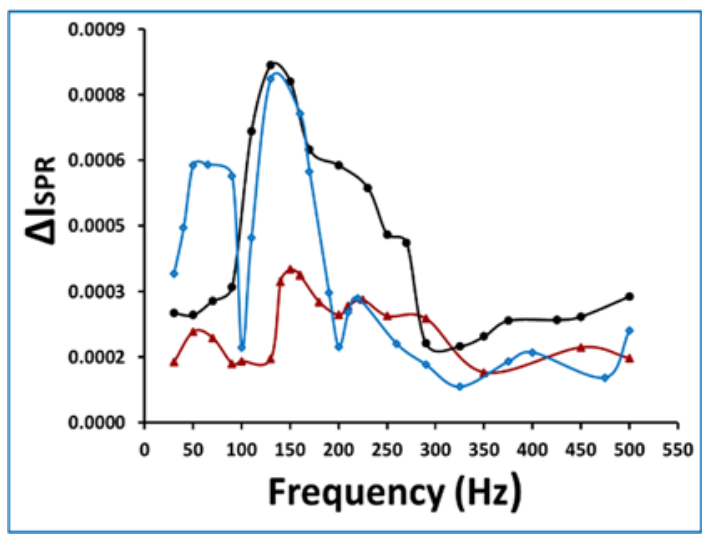

Figure 3-12: SPR optical signal, $\triangle I_{S P R}$, measured under $20 \mathrm{mV}$ AC amplitude of impedance potential modulations is plotted against angular modulation frequency for three different experiments at the same conditions, (a) without the BSA layer, whereas (b) with the presence of the BSA layer.

Figure 3-12(a) shows a clear peak with a broad distribution centered at about $250 \mathrm{~Hz}$ for each individual experiment. These peaks most likely are corresponding to the electron transfer of the cytochrome $\mathrm{c}$ protein redox process that were captured by the Cyt $\mathrm{c} \mathrm{Ab}$. In these experiments, the Cyt $\mathrm{c} A b$ was attached to CYS molecules by electrostatic interactions. This kind of interaction leads to a high-oriented antibody immobilization. Therefore, the peak that is related to the electron transfer of the cytochrome c protein redox process is expected to be narrow. In contrast, the result shows a broad peak. This broad peak can be understood if there is a chance for the antibodies to attach to the gold surface with different possible orientations in addition to adsorbing antibodies onto the CYSmodified EC-SPR platform. Therefore, these orientations can produce different electron- 
transfer rates when the cytochrome c protein attached to the antibodies, resulting in a wide range of electron-transfer rates. On the other hand, with the presence of the BSA layer, the results in Figure 3-12(b) show a clear narrow peak at about $150 \mathrm{~Hz}$. This peak could be corresponding to the redox process of the cytochrome c protein that are captured by the Cyt $\mathrm{c} \mathrm{Ab}$ after adding the BSA layer. Next, in order to measure the electron-transfer rate of the cytochrome c protein attached to the $\mathrm{Cyt} \mathrm{c} \mathrm{Ab}$, and find out the effect of BSA on the electron transfer rate, the optical data, Figure 3-12, were used. The real and imaginary components of the faradaic current densities were calculated at several frequencies, ranging from 100 to $350 \mathrm{~Hz}$ with the absence of the BSA layer, and from 100 to $200 \mathrm{~Hz}$ with the presence of the BSA layer. On the other hand, the electrical data measured by the conventional EIS technique was determined. The real and imaginary components of the impedance for the cytochrome c protein captured by Cyt c Ab are displayed in Figure 313.

(a) (b)
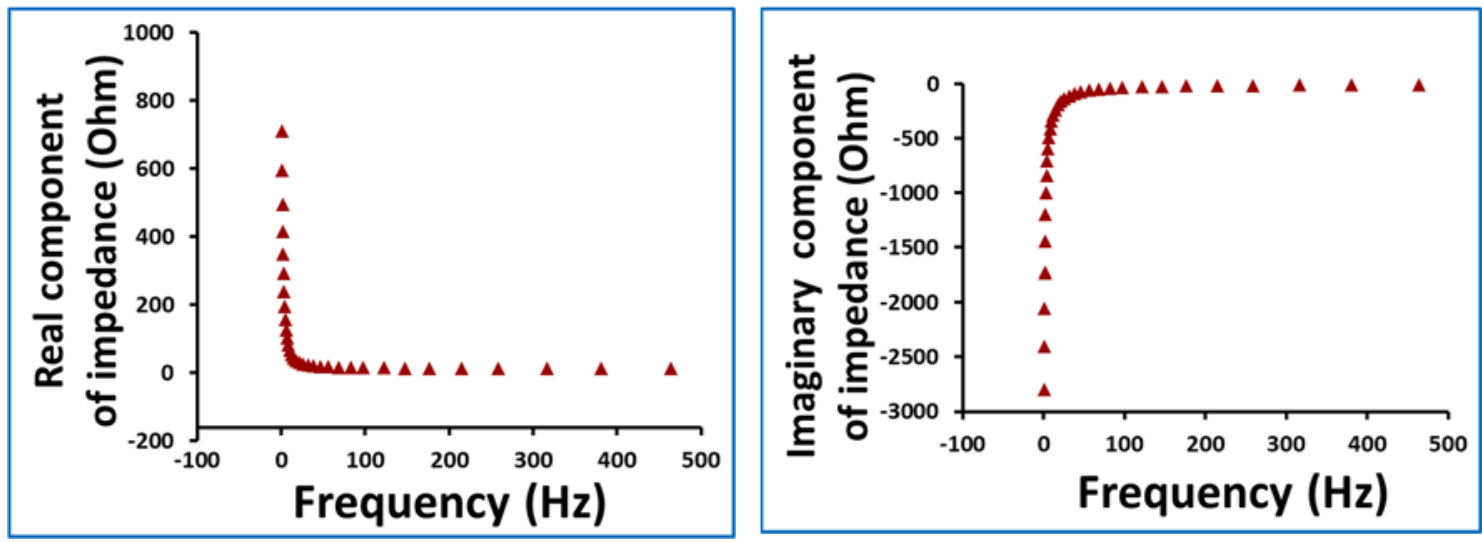

(c)

(d) 

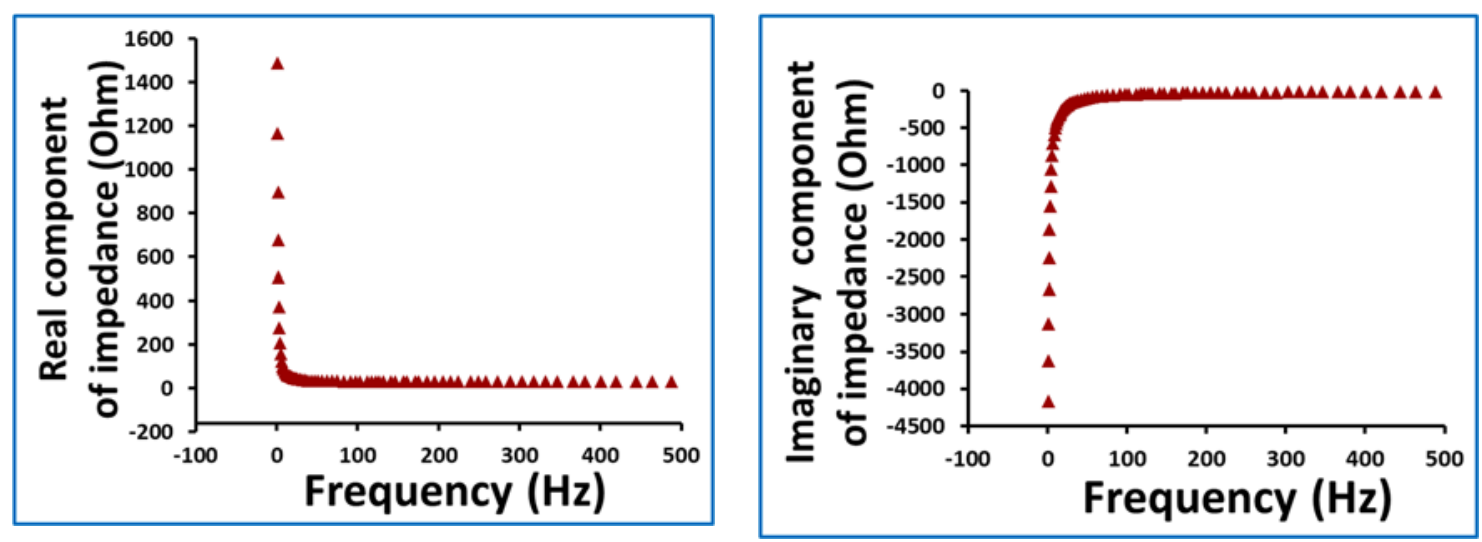

Figure 3-13: The real component of the total electrical impedance of the electrochemical flow cell (a) with cytochrome c protein captured by Cyt c Ab, while (b) is the imaginary component. (c) The real component after adding the BSA layer, while (d) is the imaginary component.

Then, the $y=\omega Y^{\prime \prime F}(\omega)$ and $x=2 Y^{\prime F}(\omega)$ were measured. Figure 3-14 displays a plot of y against $\mathrm{x}$ calculated over several modulated angular frequencies.

(a)

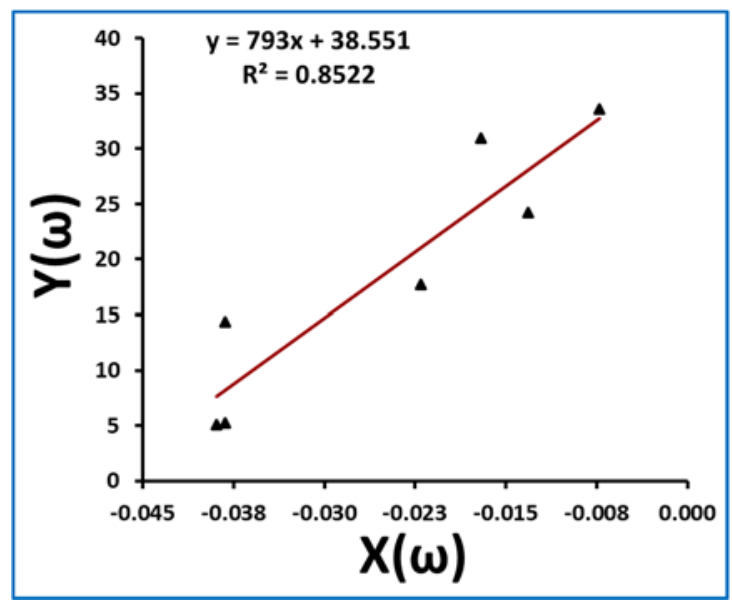

(b)

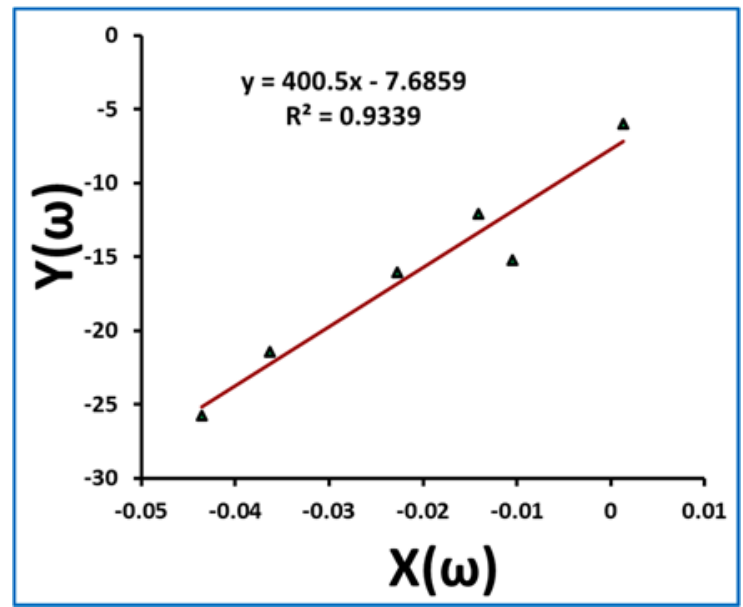

Figure 3-14: Electron-transfer rate calculation. The electron-transfer rate is represented by the slope of the linear fit, (a) without BSA layer, whereas (b) with BSA layer. 
The electron-transfer rate of the cytochrome c protein adsorbed onto Cyt c Ab/CYSmodified EC-SPR platform was extracted from the slope of the linear fitting. The average of electron-transfer rate of three experiments was $(8.1 \pm 1.5) \times 10^{2} \mathrm{~s}^{-1}$ without the BSA layer, and $(3.7 \pm 0.6) \times 10^{2} \mathrm{~s}^{-1}$ with the presence of the BSA layer. Table 3-3 summarizes the average of three results of electron-transfer rate of cytochrome $\mathrm{c}$ protein binding to different EC-SPR functionalized surfaces.

Table 3-3: The electron-transfer rates of cytochrome c protein for the different immobilization strategies.

\begin{tabular}{|l|c|}
\hline EC-SPR functionalized surface & $\begin{array}{c}\text { Electron-transfer rate of } \\
\text { cytochrome c protein }\left(\mathrm{s}^{-1}\right)\end{array}$ \\
\hline EC-SPR platform & $(1.7 \pm 0.1) \times 10^{2}$ \\
\hline MPA-modified EC-SPR platform & $(4.1 \pm 0.6) \times 10^{2}$ \\
\hline (ED-NHS)/MPA-modified EC-SPR platform & $(4.2 \pm 0.5) \times 10^{2}$ \\
\hline Cyt c Ab/CYS-modified EC-SPR platform & $(8.1 \pm 1.5) \times 10^{2}$ \\
\hline BSA/Cyt c Ab/CYS-modified EC-SPR platform & $(3.7 \pm 0.6) \times 10^{2}$ \\
\hline
\end{tabular}

The experimental results (Table 3-3) show that each immobilization strategy has a strong impact on the electron-transfer rates of cytochrome c protein binding to the EC-SPR platform. Direct immobilization of cytochrome c protein onto the EC-SPR platform shows a nearly twice-slower electron-transfer rate than the MPA-modified EC-SPR platform. This slower electron-transfer rate is expected to be due to the conformational change and the 
non-uniform distribution of cytochrome c protein on the surface, which causes substantial loss of protein activity. Our result is qualitatively consistent with previous studies 94 $9,11[147]$ in which the direct adsorption of cytochrome c protein onto metal surfaces results in poor electrochemical behavior. The increasing of the electron-transfer rate with the electrostatic adsorption indicates that the MPA provide an effective method to protect the proteins from the metal surface and then enhanced the electron-transfer rate. The cytochrome c protein with a covalent adsorption onto the MPA-modified EC-SPR platform have a slightly faster electron-transfer rate than the electrostatic adsorption. This result could be attributed to the slightly higher orientation of the immobilized cytochrome c protein on the EC-SPR platform due to the covalent bound (as mentioned earlier), which optimize the protein structure for the electron transfer processes [120, 136, 143, 148, 149]. On the other hand, the experimental results show that the specific affinity interaction between the cytochrome $\mathrm{c}$ protein and the capturing Cyt $\mathrm{c} \mathrm{Ab}$ leads to a more rapid in electron-transfer rate. The explanation for this result could be attributed to the high affinity between the capturing $\mathrm{Cyt} \mathrm{c} \mathrm{Ab}$ and the cytochrome c protein, as explained elsewhere in references $[113,149]$. Briefly, the high affinity provides a strong bound site on the surface and promotes the cytochrome $\mathrm{c}$ protein with a preferred orientation for the electron transfer processes. Thereby, the cytochrome $\mathrm{c}$ antibodies yield a significantly higher orientated layer that is adept at rapid electron exchange with the EC-SPR platform[123, 150]. Finally, the results show a large drop in the electron-transfer rate upon adding the BSA layer onto CYS-modified EC-SPR platform. The reduced electron-transfer rate constant after adding the BSA layer could be due to the blocking of some efficient electron-tunneling pathways 
as the BSA molecules have a high affinity to attached to the negatively and positively charged surfaces.

\subsection{Conclusion}

In this study, the ability of EC-SPR device for studying the electron-transfer process in molecular assemblies at electrode surfaces was demonstrated. The EC-SPR device was used to study the effect of different immobilization strategies on the electron-transfer rate of surface immobilized cytochrome c protein. Cytochrome c protein were immobilized by four various strategies: direct, electrostatic, covalent and affinity adsorption. Direct adsorption of cytochrome c protein on the EC-SPR surface shows a slower electrontransfer rate due to the non-oriented and denaturation of the cytochrome c protein on the surface. A large increasing in the electron-transfer rate with electrostatic adsorption of cytochrome c protein onto MPA indicates that the MPA provides a sensitive way to control the orientation and protect the proteins from the denaturation. A slight increase in the electron-transfer rate with a covalent bond between the proteins and the MPA-modified EC-SPR surface is due to improvements in the protein's orientation on the surface. On the other hand, with the affinity adsorption, a rapid electron-transfer rate between the cytochrome c protein and the surface was observed, due to the high affinity between the cytochrome c protein and the capturing Cyt c Ab, which offer the means for efficient orientation of the cytochrome c protein on the surface. Finally, the effect of the BSA layer on the electron-transfer rate between cytochrome $\mathrm{c}$ protein that were captured by $\mathrm{Cyt} \mathrm{c} \mathrm{Ab}$ and the EC-SPR surface was examined. The electron-transfer rate dropped substantially after adding the BSA layer due to blocking an effective electron-transfer pathways. This study shows that each immobilization strategy has a strong impact on the electron-transfer 
rates of cytochrome c protein binding to the surface. The results could be a key for increasing the sensitivity and the efficiency in many applications, such as bio-electronics devices, solar cells and biomedical applications. 


\section{CHAPTER 4}

\section{INFLUENZA VIRUS DETECTION WITH A FUNCTIONALIZED EC-}

\section{SPR PLATFORM}

\subsection{Introduction}

The potential to create a biosensing technology with rapid, highly specific, and outstandingly sensitive for diagnosis and identification of causative agents of human pathogens as viruses, bacterial, viral, fungal is vitally important not only to acute clinical diagnosis and care of patients but also for epidemiologists to prevent outbreaks and maintain public health. Influenza viruses, for example, are the leading causes of infectiousdisease related death in the world. The continuous antigenic changes of the influenza viruses and their capability to be a human-human transmission have caused increasing concerns about viruses' outbreak around the world. Influenza viruses' outbreak not only causes epidemics but also could lead to a global risk as well. For example, in the last century, three pandemics of respiratory disease caused by coronavirus outbreaks have emerged from animals to cause a global outbreak of disease. Coronaviruses are a large family of viruses that circulate among many different animals including camels, pigs, cattle, cats, and bats. Some of these viruses such as with MERS-CoV and SARS-CoV can transmission from animals to infect people and then cause serious disease. 
Presently, a new novel coronavirus called COVID-19 causes an international concern over a global outbreak of the virus. According to the World Health Organization (WHO), coronavirus is infecting people and sustainably spreading between people in most countries worldwide. The virus outbreak was characterized as a pandemic on March 11,2020, and become one of the biggest threats to the global economy and financial markets that heavily impact business and travel[151]. Therefore, from the first day of the discovery of COVID19, the researchers have initiated studies to better understand the virus to develop diagnostics, therapeutics, and vaccines that can stop or reduce the impact of the virus spread. Currently, global efforts are focusing on decreasing the spread of the virus and the next couple of months are going to be absolutely critical in the global battle against the COVID-19. Therefore, early diagnosis and identification of influenza viruses are crucial for epidemiologists to prevent outbreaks in closed environments and to better formulate annual flu vaccines to correctly protect against the most pertinent strains of influenza. The most virulent forms of influenza virus, type A (and related subtypes) and type B are easily distinguished through immune detection technology due to the presentation of different subtypes of the two main influenza antigens: hemagglutinin (HA) and viral neuraminidase (NA) [152]. Both proteins are found on the exterior portion of the virus surface and are therefore amenable to $\mathrm{Ab}$ trapping assays[153]. A biosensing technology that is compatible with multiplex detection and displays high sensitivity and specificity would therefore be an ideal solution for rapid detection and identification of prevalent influenza subtypes[154].

However, in chapter 1 an inexpensive and easy-to-use transduction platform based on electrochemically modulated SPR configuration that offers a simpler path towards an 
arrayed detection technology with multiplexed interrogation confined to a small device footprint was developed. Moreover, the ability of a potential modulation to drive the SPR signal and create an optical output signal of a redox reaction through different molecular immunolayer assemblies was demonstrated in chapter 2.

In this chapter, a novel viral pathogen immunosensor technology, based on the electrochemical modulation of the optical signal from a surface plasmon wave, interacting with a redox dye reporter is developed and described. The device is formed by incorporating a sandwich immunoassay onto the surface of a plasmonic device mounted in a micro-electrochemical flow cell. A methylene blue (MB) dye was used as a redox-active probe since it displays a reversible change in the complex refractive index throughout a reduction-oxidation transition, which generates an optical signal that can be electrochemically modulated and detected at high sensitivity. The hemagglutinin (HA) protein from the $\mathrm{H} 5 \mathrm{~N} 1$ avian influenza A virus was targeted in order to demonstrate the capability of our biosensing technology for the detection and quantification of a critical influenza antigen.

\subsection{The EC-SPR Methodology for Detection of Influenza Virus Immunosensing}

The sensing strategy using EC-SPR is illustrated schematically in Figure 1-4. 


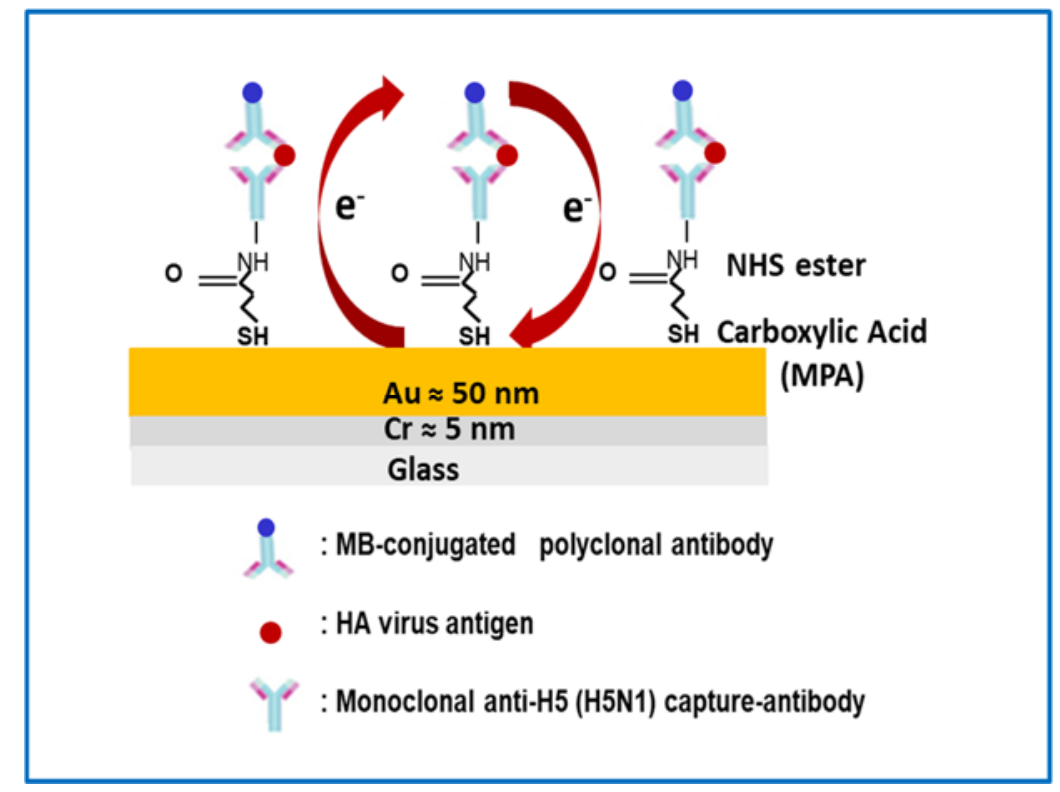

Figure 4-1: A glass slide coated with a $5 \mathrm{~nm}$ chromium adhesion layer followed by a 50 nm layer of gold to create a biosensor surface. A molecular assembly formed by layers of MPA, EDC, and NHS were used to functionalize the biosensor interface.

The detection principle is based on the interaction between the monoclonal anti-H5 (H5N1) capture $\mathrm{Ab}(250 \mu \mathrm{g}, 2 \mathrm{mg} / \mathrm{ml})$, the hemagglutinin (HA) virus antigen $(200 \mu \mathrm{g} / \mathrm{ml}, 0.5 \mathrm{ml})$, and the MB-labeled secondary Ab. Once, and only if the target antigen is bound to the device surface, it promotes the capturing of a secondary polyclonal $\mathrm{Ab}$ that has been conjugated with the redox-active MB dye. Then, by applying a modulated electric potential to force the oscillation of the MB across one of its oxidation states, a modulated optical signal in reflection is created and detected. Such methodology exclusively detects an optical signal that is electrically-modulated and constrained to the analytical interface, which provides a means to significantly reduce the effects of background signals that come from the substrate or fluid media. Additional selectivity in the analytical signal is obtained 
by modulating the applied potential around the formal potential of the redox probe and by choosing the laser wavelength to be centered at the optical transition of the dye.

\subsection{Optimization of the EC-SPR Device}

Two important parameters have a significant impact on the final EC-SPR device performance in the development of an efficient immunoassay method: I) the uniformity and density of the capturing Ab layer on the active interface, and II) the optical signal differences between the reduced and oxidized states of the redox-active probe under potential modulations. In this section, a capturing $\mathrm{Ab}$ and MB-labeled secondary $\mathrm{Ab}$ were deployed to determine the best parameters in the multiple steps of the biomolecular functionalization of EC-SPR platform for detection of the specific antigen.

\subsubsection{The Optimal Primary Ab Concentration}

The capturing Ab purchased from Santa Cruz Biotechnology (ab82455abcam, 250 $\mu \mathrm{g}, 2 \mathrm{mg} / \mathrm{mL}$ ) was used to characterize and optimize the monolayer assemblies of functionalizing the surface with the primary Ab. Different concentrations of the capture $\mathrm{Ab}(0.5 \mu \mathrm{g} / \mathrm{ml}, 1 \mu \mathrm{g} / \mathrm{ml}$, and $2 \mu \mathrm{g} / \mathrm{ml})$ were investigated. The same setup of EC-SPR illustrated in Figure 2-2, chapter 2, was employed for angular reflectance experiments. Typical SPR reflectance curves were determined to investigate the three concentrations for reaching enhanced biological functionality at the active device interface. The MPAmodified gold surface was mounted in homemade micro-electrochemical flow cell with a three-electrode configuration. Then the micro-electrochemical flow cell was mounted on a rotation stage (quadra chek 100 rotation stage) to precisely control the incident angle. A PBS buffer solution ( $\mathrm{pH}=7.4)$ of $2 \mathrm{mM}$ of EDC and $5 \mathrm{mM}$ of NHS was incubated in the flow cell for 60 minutes to convert the terminal carboxylic groups of the MPA to active 
NHS esters, then the capturing Ab was incubated in the flow cell for 30 minutes. After that, the flow cell was rinsed with a PBS buffer solution to remove unbound species from the surface. Finally, the incident angle was scanned by rotating the stage and the reflected optical intensity at different angles of incidence were monitored by a power meter (Newport, model 193). Figure 4-2 shows the measured SPR reflectance curves against the effective refractive index inside the prism for a PBS buffer solution and three different concentrations of capturing $\mathrm{Ab}$.

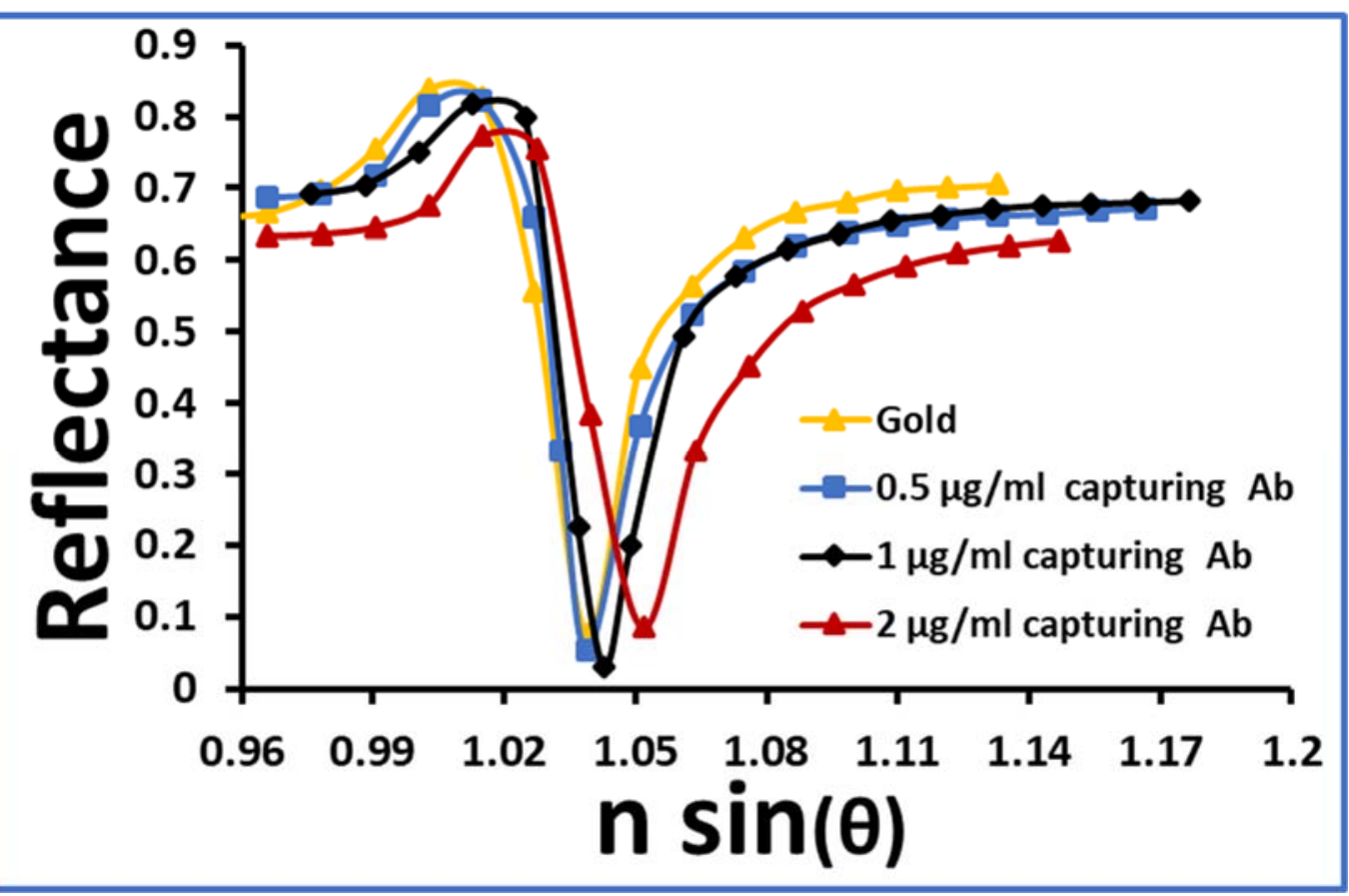

Figure 4-2: The SPR reflectance curves against the effective refractive index inside the prism for a PBS buffer solution and three different concentrations of capturing Ab.

The yellow curve in Figure 4-2 shows the SPR reflectance for the PBS buffer solution, while the blue, black, and red curves show the SPR reflectance for the three concentrations of the capturing $\mathrm{Ab}$. The results show that after injecting the $0.5 \mu \mathrm{g} / \mathrm{ml}$ solution of capturing $\mathrm{Ab}$, the position of minimum reflectance is about the same as obtained for the PBS buffer 
solution. This indicates a small amount of the capturing $\mathrm{Ab}$ was attached onto the activated MPA-modified gold surface. On the other hand, and after injecting the $1 \mu \mathrm{g} / \mathrm{ml}$ and $2 \mu \mathrm{g} / \mathrm{ml}$ solutions of capturing $\mathrm{Ab}$, a shift in the position of minimum reflectance was observed. From these results, it could be confirmed that the capturing Ab layer was formed on the activated MPA using the $1 \mu \mathrm{g} / \mathrm{ml}$ and $2 \mu \mathrm{g} / \mathrm{ml}$ solutions of capturing Ab. Next, a cyclic voltammetry (CV) technique was applied to examine the effect of the capturing $\mathrm{Ab}$ concentration on the optical signal differences between the reduced and oxidized states of the redox-active probe under potential modulations. MB-labeled a polyclonal anti-H5 (H5N1) was used. The sensor surface was mounted on an electrochemical flow cell and the optical data under CV potential modulation from -0.45 to $0.2 \mathrm{~V}$ at a scan rate of $0.02 \mathrm{~V} / \mathrm{s}$ was collected with a PBS buffer solution (without the presence of MB-labeled secondary $\mathrm{Ab}$ in the flow-cell). Then the optical data under the same $\mathrm{CV}$ potential modulation was collected with the presence of three concentrations of the MB-labeled secondary Ab (0.5 $\mu \mathrm{g} / \mathrm{ml}, 1 \mu \mathrm{g} / \mathrm{ml}$ and $2 \mu \mathrm{g} / \mathrm{ml}$ ) respectively, in the flow-cell. Finally, the optical signal, $I$, was determined by subtracting the optical signal of the MB-labeled secondary Ab by the optical signal of the PBS buffer solution under cyclic voltammetry. Figure 4-3 shows the optical signal, $I$, under cyclic voltammetry for the solution of MB-labeled polyclonal $\mathrm{Ab}$ at concentration of (a) $0.5 \mu \mathrm{g} / \mathrm{ml}$, (b) $1 \mu \mathrm{g} / \mathrm{ml}$, and (c) $2 \mu \mathrm{g} / \mathrm{ml}$. 
(a)

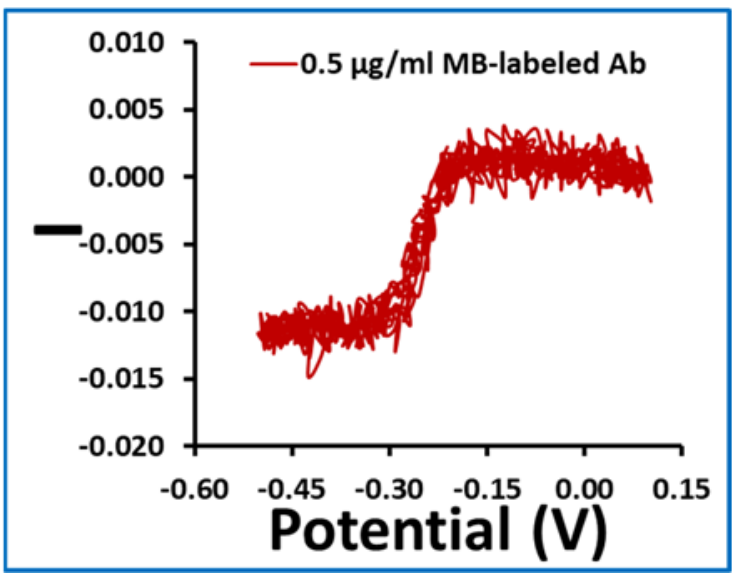

(b)

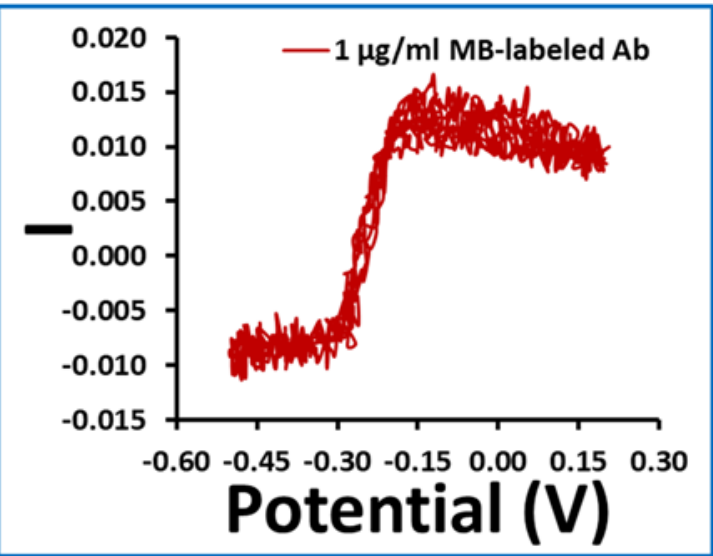

(c)

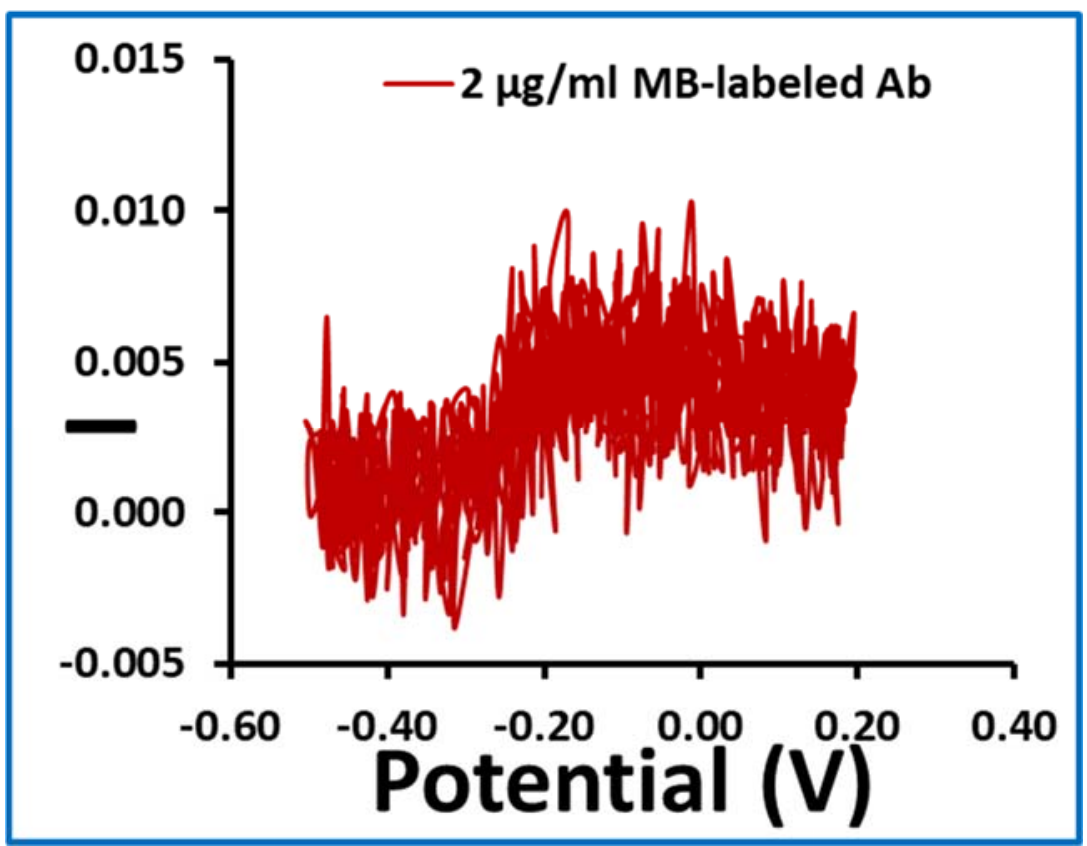

Figure 4-3: Optical signal ,I, under a CV scan for the MB-labeled polyclonal Ab immobilized onto SPR platform with scan rate of $0.02 \mathrm{~V} / \mathrm{s}$ at concentration of (a) $0.5 \mu \mathrm{g} / \mathrm{ml}$ , (b) $1 \mu \mathrm{g} / \mathrm{ml}$, and (c) $2 \mu \mathrm{g} / \mathrm{ml}$. 
The results in (a) and (b) with the concentration of $0.5 \mu \mathrm{g} / \mathrm{ml}$ and $1 \mu \mathrm{g} / \mathrm{ml}$ respectively, show a reversible and clear transition of the optical signal, $I$, as the modulation potential crosses the formal potential of the MB (approximately at $-0.25 \mathrm{~V}$ ). The optical signal difference, $\Delta I$, were estimated to be 0.011 and 0.02 for $0.5 \mu \mathrm{g} / \mathrm{ml}$ and $1 \mu \mathrm{g} / \mathrm{ml}$ respectively. While with a concentration of $2 \mu \mathrm{g} / \mathrm{ml}$, the transition of the optical signal, $I$, is very small $(\Delta I=0.003)$, this small transition is possibly due to form a thick layer of MB-labeled polyclonal $\mathrm{Ab}$ on the surface, that is blocking the electron transfer between the MB and the gold surface. From this data, the most optimal MB-labeled secondary Ab concentration is $1 \mu \mathrm{g} / \mathrm{ml}$, which gives the highest magnitude of the optical signal difference, $\Delta I$, between the two oxidation states of the MB. Based on this result and the result obtained from section 4.3.1, the monoclonal capture $\mathrm{Ab}$ concentration of $1 \mu \mathrm{g} / \mathrm{ml}$ was chosen as an optimal concentration for optimizing the EC-SPR surface to fully cover the active device interface and keep a strong difference $\Delta I$ of the optical response.

\subsubsection{Optimization of the Analytical Signal}

The principle of the EC-SPR sensor relies on a strong difference $\Delta I$ of the optical response between the reduced and oxidized states of the conjugated MB ester in the full immunoassay assembly. However, because the conjugated MB ester has similar spectroscopic properties as the pristine MB molecule under the redox process, the original MB molecule was used for searched for the ideal SPR configuration to optimize the ECSPR response with the highest optical signal , $\triangle I$. Figure 4-4 shows typical SPR reflectance curves against the effective refractive index inside the prism for a PBS buffer solution and a 2.6 $\mu \mathrm{M} \mathrm{MB}$ solution, where both samples were measured at two electrical potentials 
applied to the working electrode: $-0.35 \mathrm{~V}$ (which corresponds to a reduced state of $\mathrm{MB}$ ) and $0.05 \mathrm{~V}$ (an oxidized state of MB).

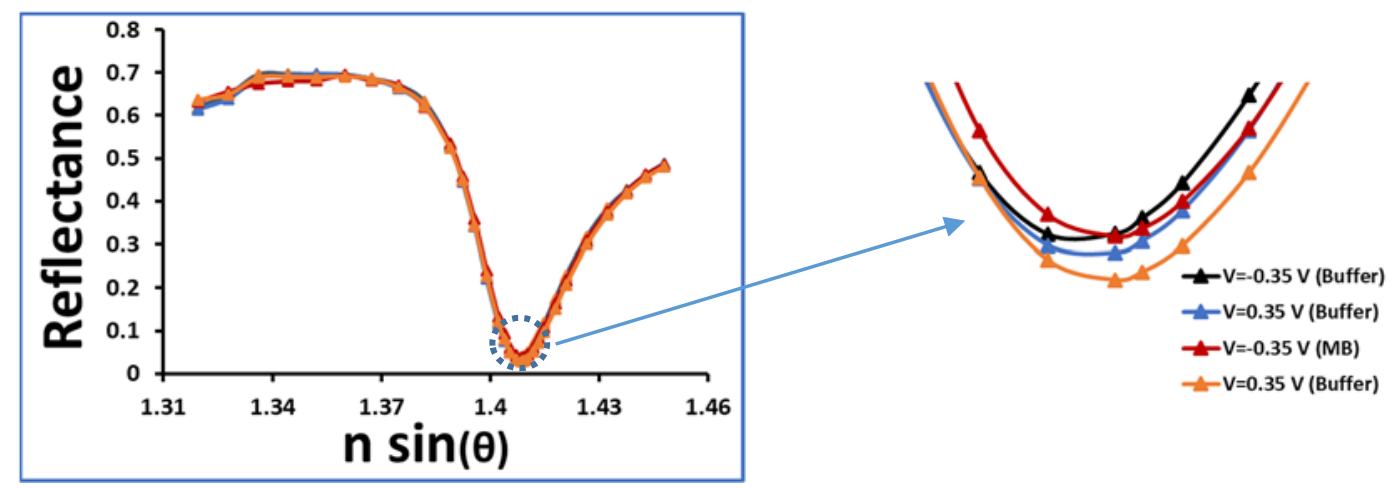

Figure 4-4: SPR reflectance for the PBS buffer and MB solutions at $-0.35 \mathrm{~V}$ and $0.35 \mathrm{~V}$.

The black and blue curves in Figure 4-4 show the SPR reflectance for the buffer solution at $-0.35 \mathrm{~V}$ and $0.05 \mathrm{~V}$, respectively. After injecting the $\mathrm{MB}$ solution at $-0.35 \mathrm{~V}$ potential (red curve), the position of minimum reflectance has shifted to a higher value of effective refractive index. However, the value of minimum reflectance is about the same as obtained for the buffer solution at this applied potential (black curve). Such behavior confirms that the change is mainly due to a modification in the real part of the refractive index near the surface plasmon wave, as MB molecules replace water molecules. The almost-constant value in the minimum of reflectance indicates the high transparency of the MB molecule at $-0.35 \mathrm{~V}$ (MB is highly transparent in its reduced state). On the other hand, at the $0.05 \mathrm{~V}$ potential (orange curve) a significant change in the value of the reflectance minimum of the MB solution was observed (when compared to the buffer solution data at that potential, blue curve), which is consistent with significant changes in the imaginary part of the refractive index of the $\mathrm{MB}$ molecule. These results confirm that the MB absorption change 
under the redox process is present in our experiment and can potentially be deployed as a useful probe.

Next, an optical signal, $I$, was determined by subtracting the reflectance of the MB solution by the reflectance of the PBS buffer solution. Such an operation was performed at each effective refractive index and at two applied potentials $(-0.35$ and $0.05 \mathrm{~V})$ corresponding to the reduced and oxidized states of the MB molecule, respectively. The optical signal difference, $\Delta I$, between the two oxidation states of the MB sample was then determined against the effective refractive index (Figure 4-5, black trace), while the blue curve (y-axis on the right side of the plot) provides the conventional SPR reflectance of the platform submersed in buffer solution.

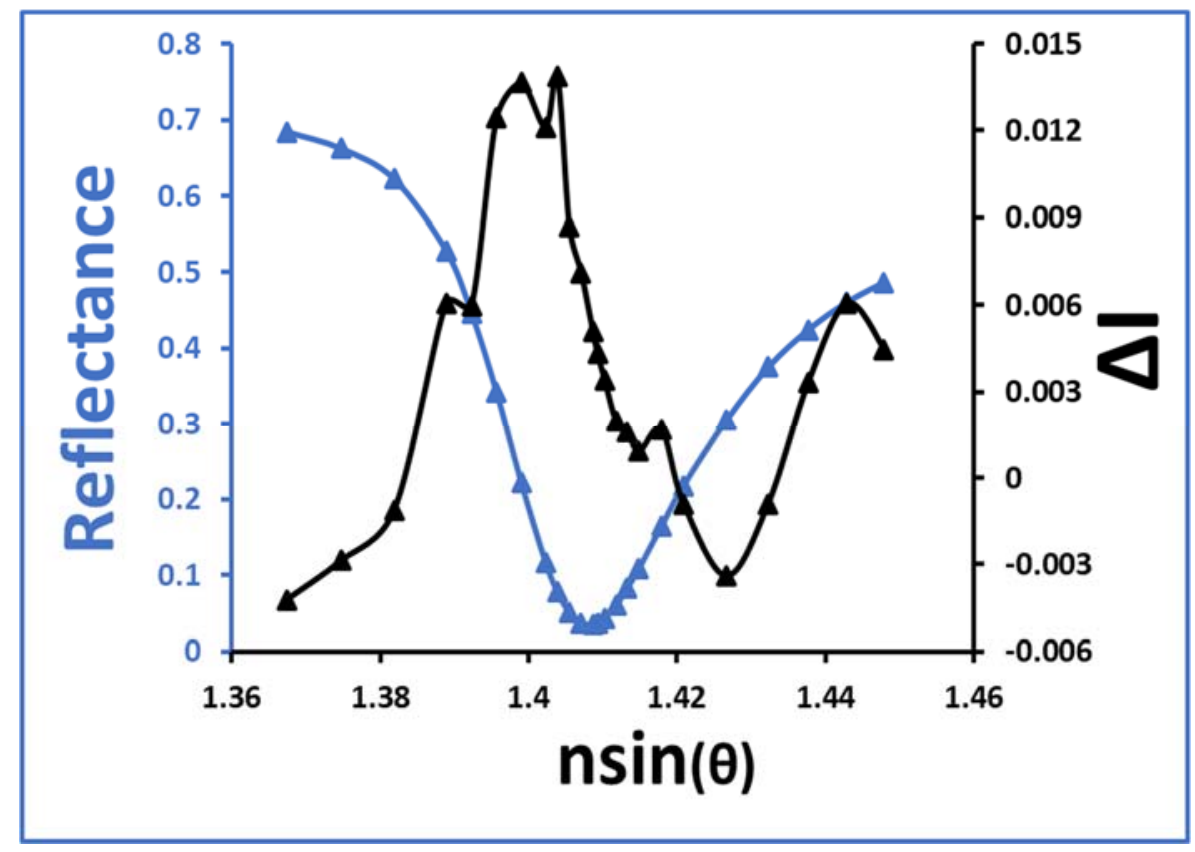

Figure 4-5: Left y-axis shows the SPR reflectance for the PBS buffer solution, while the right $y$ - axis shows the difference in the optical signal , $\Delta I$, between the oxidized state and the reduced state of the MB molecule. 
These results show that the highest magnitude of the optical signal difference, $\Delta I$, is observed in the linear region on the left side of the resonant angle of the conventional SPR curve. These experimental results come from changes in both the real and imaginary parts of the refractive index of the MB molecules when undergoing a redox process, and they define the best fixed angle (i.e., ideal effective refractive index) to implement EC-SPR measurements that will maximize the amplitude of the optical modulation when an electrochemical modulation is applied.

\subsection{Virus Protein Detection with Immunoassay on the EC-SPR Device}

Once the monolayer assemblies of the capturing $\mathrm{Ab}$ and the optical response of the EC-SPR device were characterized and optimized, a proof-of-principle demonstration on the capabilities of the EC-SPR sensor for detection and quantification of a critical influenza antigen was performed. A hemagglutinin (HA) protein from the $\mathrm{H} 5 \mathrm{~N} 1$ avian influenza A virus was targeted. HA is a ubiquitous glycoprotein component of the influenza virus capsid responsible for binding the virus to host cells, and is thus a useful antigen for our assay.

\subsubsection{Sample Preparation and Functionalization Protocol of the EC-SPR Surface with an Immunoassay Targeting an Influenza Virus Antigen}

In order to perform the principle of the EC-SPR sensor, chromium $(5 \mathrm{~nm})$ and gold films (50 nm) were deposited on soda lime glass slides (VWR, 48300-048) using a DC magnetron sputtering technique (Nano-Master). Then, the sensor interface of the goldcoated glass slide was sequentially cleaned in an ultrasonic bath with deionized (DI) water, acetone and ethanol for 10 minutes in each solution, and then was gently dried under $\mathrm{N}_{2}$ gas. After those steps, the device was immediately immersed into a $10 \mathrm{mM}$ solution of 
MPA in ethanol for 24 hours. After rinsing the sensor surface with ethanol and DI water, and drying it with $\mathrm{N}_{2}$ gas, the device was placed in a PBS buffer solution $(\mathrm{pH}=7.4)$ of 2 $\mathrm{mM}$ of EDC and $5 \mathrm{mM}$ NHS for 1 hour to convert the terminal carboxylic group to an active NHS ester $[155,156]$. Then a monoclonal anti-HA (H5N1) capture Ab (purchased from Santa Cruz Biotechnology (ab82455abcam, $250 \mu \mathrm{g}, 2 \mathrm{mg} / \mathrm{mL}$ )) was immobilized on the sensor surface by covalently binding it to the MPA by immersing the sensor surface in a $1 \mu \mathrm{g} / \mathrm{ml}$ capture antibody in PBS buffer solution at $4{ }^{\circ} \mathrm{C}$ overnight. Finally, the sensor surface with the immobilized capture Ab was rinsed with PBS buffer to remove any unbounded antibodies and then stored at $4{ }^{\circ} \mathrm{C}$. Figure 4- 6 shows the schematic diagram of the monoclonal anti-H5 (H5N1) capture Ab immobilized onto gold surface modified with MPA molecules and activated with EDC/NHS.
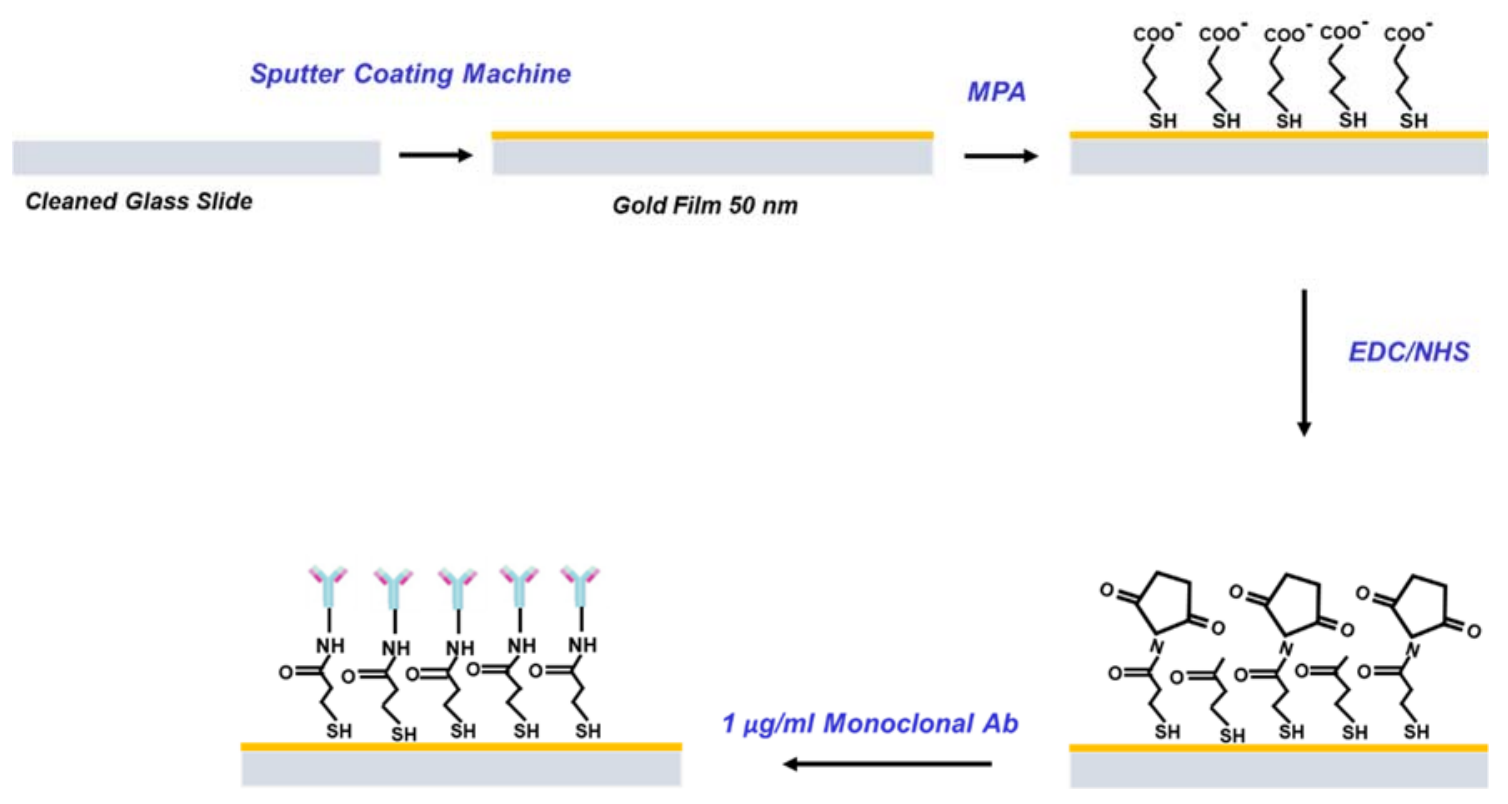

Figure 4-6: Schematic diagram of the monoclonal anti-H5 (H5N1) capture Ab immobilization on the gold surface modified with MPA molecules and activated with EDC/NHS. 


\subsubsection{EC-SPR Biosensor Setup}

In order to demonstrate the EC-SPR immunosensor, the experimental setup used was the same as described in chapter 3 and illustrated in Figure 4-7.

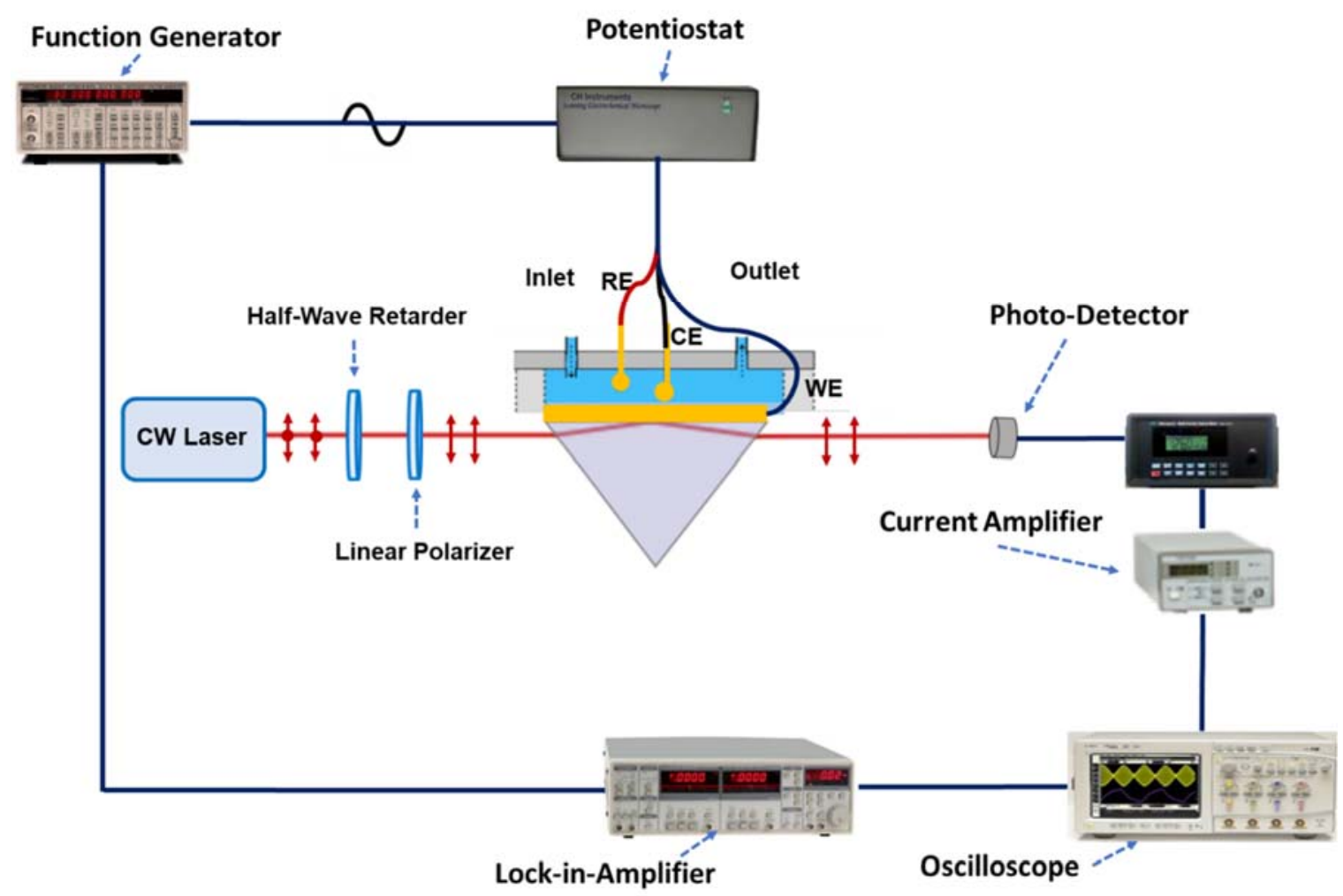

Figure 4-7:Schematic diagram of EC-SPR setup with a potentiostat for electrical control, laser light, polarizer and half-wave retarder for P polarized light. A detector, current amplifier, lock-in amplifier and oscilloscope were used for data collection.

A CW laser (Obis from Coherent) at the wavelength of $685 \mathrm{~nm}$ was operated at $40 \mathrm{~mW}$. The experimental setup illustrated in Figure 4-7 was used for two different measurements: I) cyclic voltammetry (CV) modulation measurements, for which the electrically modulated reflected optical signal was monitored at a fixed angle by the power meter and transferred to an oscilloscope (Agilent, DSO8104A Infiniium).II) AC potential modulation 
measurements, for which a function generator (DS345, Stanford Research Systems) was connected to the potentiostat to apply a sinusoidal potential modulation to drive the oscillation of the MB across its redox state. The modulated optical signal of the reflected light was monitored at a fixed incident angle by the power meter, which was connected to a low-noise current pre-amplifier and then delivered to a lock-in amplifier (SR830 DSP, Stanford Research Systems), where the magnitude and the phase of the analytical signal were collected and recorded by the oscilloscope.

\subsubsection{Cyclic Voltammetry (CV) Technique}

For a proof-of-principle demonstration on the capabilities of the EC-SPR sensor for detection and quantification of a critical influenza antigen, a monoclonal anti-HA (H5N1) capture $\mathrm{Ab}$ was immobilized on the sensor. The sensor surface was mounted on an electrochemical flow cell and stabilized with a PBS buffer solution under CV potential modulation from -0.4 to $0.2 \mathrm{~V}$ at a scan rate of $0.02 \mathrm{~V} / \mathrm{s}$. Two measurements are highlighted below: in the first measurement, a $50 \mu \mathrm{L}$ solution of MB labeled polyclonal antibody at a concentration of $10 \mu \mathrm{g} / \mathrm{ml}$ was incubated in the flow cell for 30 min before rinsing with a PBS buffer. In the second measurement, a $50 \mu \mathrm{L}$ solution of $200 \mathrm{ng} / \mathrm{ml} \mathrm{HA}$ protein of the H5N1 influenza virus was prepared in $0.2 \mathrm{M}$ PBS buffer solution and incubated in the flow cell for 30 min before rinsing with the PBS buffer solution. This step was followed by injecting a $50 \mu \mathrm{L}$ solution of MB-labeled polyclonal Ab solution at a concentration of 10 $\mu \mathrm{g} / \mathrm{ml}$ and incubating for 30 min before the flow cell was rinsed with PBS buffer solution to remove any unbound MB-labeled polyclonal Ab. Figure 4-8 shows the optical signal, $I$, under cyclic voltammetry for the two measurements described above: in the presence of the $200 \mathrm{ng} / \mathrm{ml} \mathrm{HA}$ virus antigen (black trace) and in the absence of the HA virus antigen 
(red trace), where each trace was several overlapping cycles of the applied electric potential.

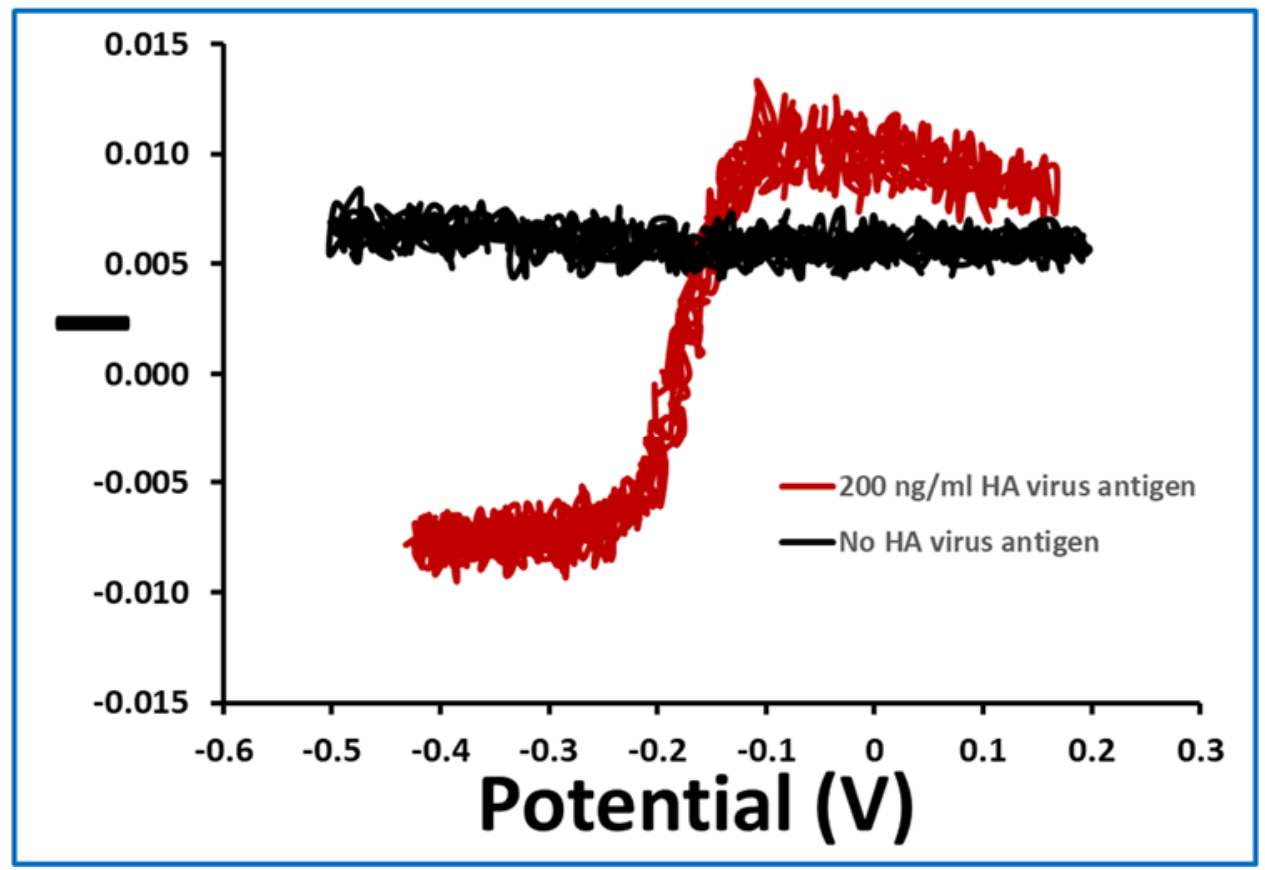

Figure 4-8: Optical signal, I, under a CV scan for the full immunoassay sandwich at scan rate of $0.02 \mathrm{~V} / \mathrm{s}$. The red trace shows the data in the absence of HA virus antigen, while the black trace represents the data in the presence of HA virus antigen.

The results in the presence of antigen show a reversible and sharp transition of the optical signal, $I$, as the modulation potential crosses the formal potential of the redox probe and indicates the presence of the HA virus antigen, which is responsible for capturing and retaining the active redox probe $(\mathrm{MB})$ at the device interface. In contrast, the absence of HA virus antigen (red trace) translates into an almost constant optical signal, $I$. This control experiment also confirms that the non-specific adsorption of the MB-labeled polyclonal $\mathrm{Ab}$ on the device interface was successfully kept to negligible levels. Above all, these 
results unambiguously confirm the ability of the EC-SPR platform to detect the presence of the HA virus antigen through the modulated optical signal under a CV scan.

\subsubsection{AC Voltammetric Technique}

The data in section 4.4 .3 shows that, at least for a concentration of $200 \mathrm{ng} / \mathrm{ml}$ of the HA virus antigen solution, the EC-SPR platform under CV potential modulation can be used to detect the target analyte. However, to reach detectability at much lower analyte concentrations, increase the sensitivity and selectivity route for detection of binding events, better interrogation schemes should be implemented. For this purpose, an AC voltammetric modulation was deployed to drive the redox process and consequently the optical signal with a much-improved signal-to-noise ratio while delivering a faster transduction time. In order to study how the AC voltammetric modulation technique can help to improve the optical signal and reduce the effect of the non-specific adsorption optical signal, two experiments were deployed. First, an AC voltammetric modulation was deployed to drive the redox process of the MB-labeled polyclonal Ab adsorbed onto MPA-modified sensor surface. Second, an AC voltammetric modulation was deployed with the full immunoassay sandwich. In both experiments, the resonance frequency (modulation frequency), which is essentially connected to the electron transfer kinetics of the redox process occurring on the electrode surface and the formal potential of the redox probe (MB) were experimentally determined. To do so, first, the formal potential of the MB-labeled polyclonal Ab adsorbed onto the MPA was determined. The optical signal, I, on the EC-SPR platform for the MBlabeled polyclonal $\mathrm{Ab}$ was first collected under $\mathrm{CV}$ potential modulation. The baseline optical signal was collected after the platform was functionalized with the activated MPA and the reporter optical signal was collected after the incubation (and rinsing) of the MB- 
labeled polyclonal $\mathrm{Ab}$ at a concentration of $1 \mu \mathrm{g} / \mathrm{mL}$. Figure 4-9 (black trace) shows the optical signal, $I$, under a CV scan for the MB-labeled polyclonal Ab adsorbed onto the MPA at scan rate of $0.02 \mathrm{~V} / \mathrm{s}$.

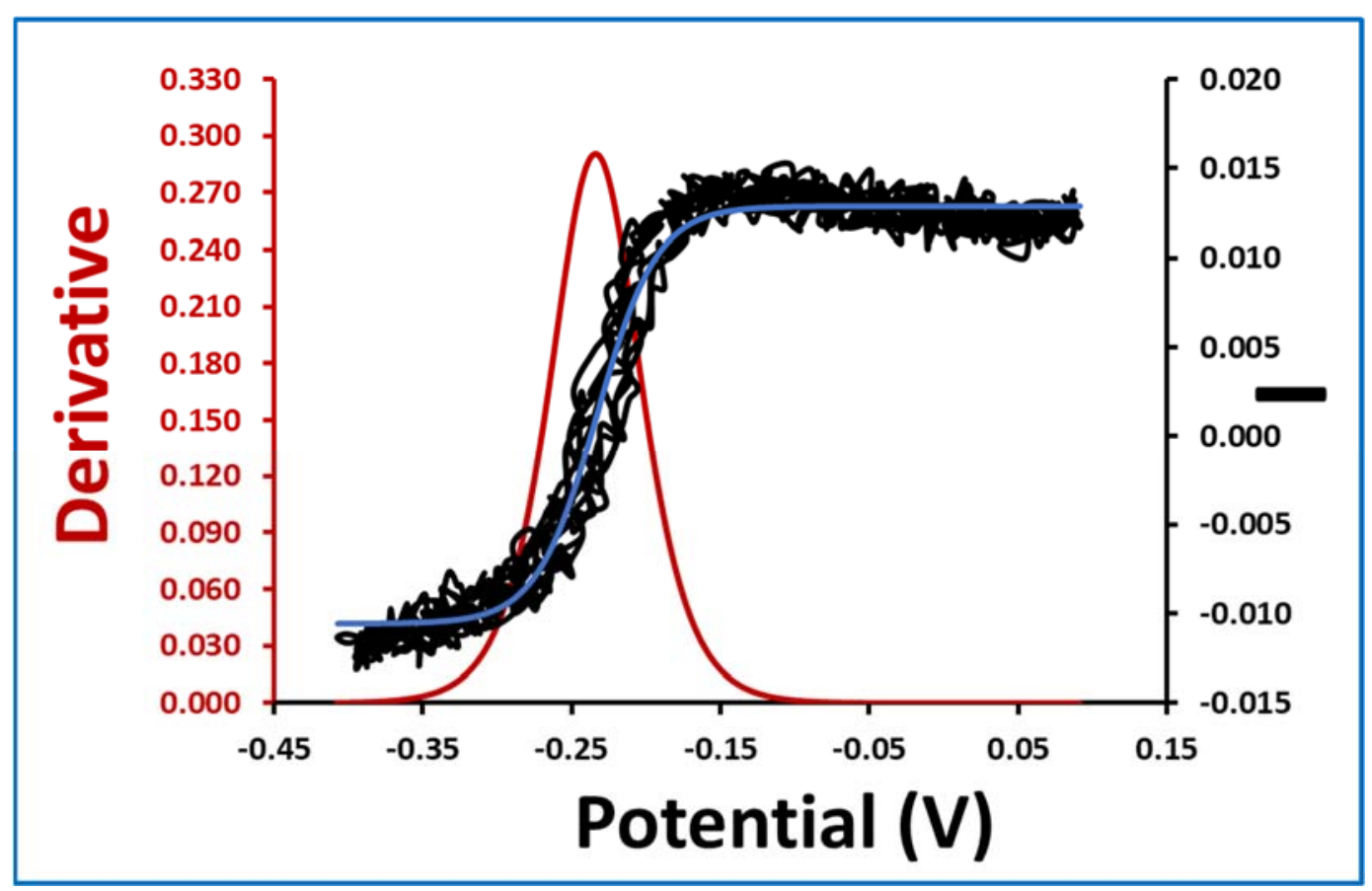

Figure 4-9: Optical signal, $I$, under a CV scan for the MB-labeled polyclonal Ab adsorbed onto the activated MPA at scan rate of $0.02 \mathrm{~V} / \mathrm{s}$ (black trace). The blue trace shows the fitting of the optical signal, while the red trace represents the derivative of the fitting of the optical signal.

The blue trace represents the fitting of the optical signal, $I$. The red curve (y-axis on the left side of the plot) provides the derivative of the fitting of the optical signal, $I$; this trace was used to approximate the formal potential to be $-0.23 \mathrm{~V}$. Second, the resonance frequency of the MB-labeled polyclonal Ab adsorbed onto the activated MPA-modified sensor surface was determined. A sinusoidal potential modulation was applied on the ECSPR sensor with the MB-labeled polyclonal Ab adsorbed onto the activated MPA-modified 
sensor surface at the formal potential $(-0.25 \mathrm{~V})$, and several frequencies ranging from 25 to $500 \mathrm{~Hz}$ were measured. The SPR optical signal, $\triangle I S P R$, was determined by subtracting the baseline SPR optical signal, which was taken after stabilizing the activated MPAmodified sensor surface from the SPR optical signal collected after the incubation (and rinsing) of the MB-labeled polyclonal Ab. The SPR optical signal, $\triangle I_{S P R}$, versus the frequency of the sinusoidal potential modulation is shown in Figure 4-10.

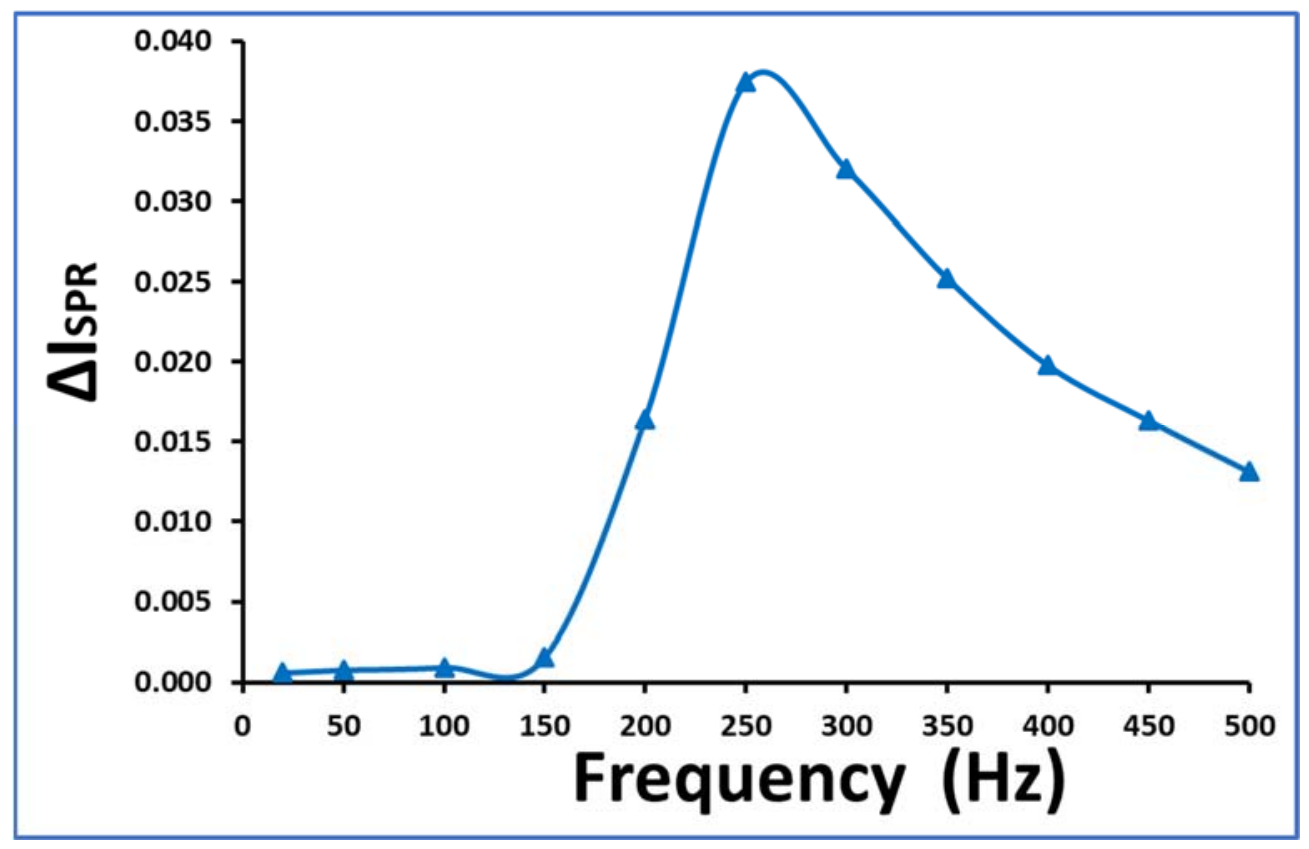

Figure 4-10: SPR optical signal, $\triangle \mathrm{ISPR}_{\mathrm{SP}}$, measured under AC potential modulation with the MB-labeled polyclonal Ab adsorbed onto the activated MPA-modified sensor surface at the DC bias potential of $-0.25 \mathrm{~V}$.

A peak centered at about $150 \mathrm{~Hz}$ is related to the electron transfer of the MB-labeled polyclonal $\mathrm{Ab}$ adsorbed onto the activated MPA-modified sensor surface. Next, the resonance frequency (modulation frequency) and the formal potential of the redox probe (MB) with the full immunoassay sandwich were examined. The data under cyclic 
voltammetry for the full immunoassay sandwich was used to precisely determined the formal potential of $(\mathrm{MB})$ by calculating the derivative of the optical signal, $I$, with respect to the applied potential. Figure 4-12 (black trace) shows the optical signal, $I$, under a CV scan for the full immunoassay sandwich at scan rate of $0.02 \mathrm{~V} / \mathrm{s}$, while the blue trace represents the fitting of the optical signal, $I$.

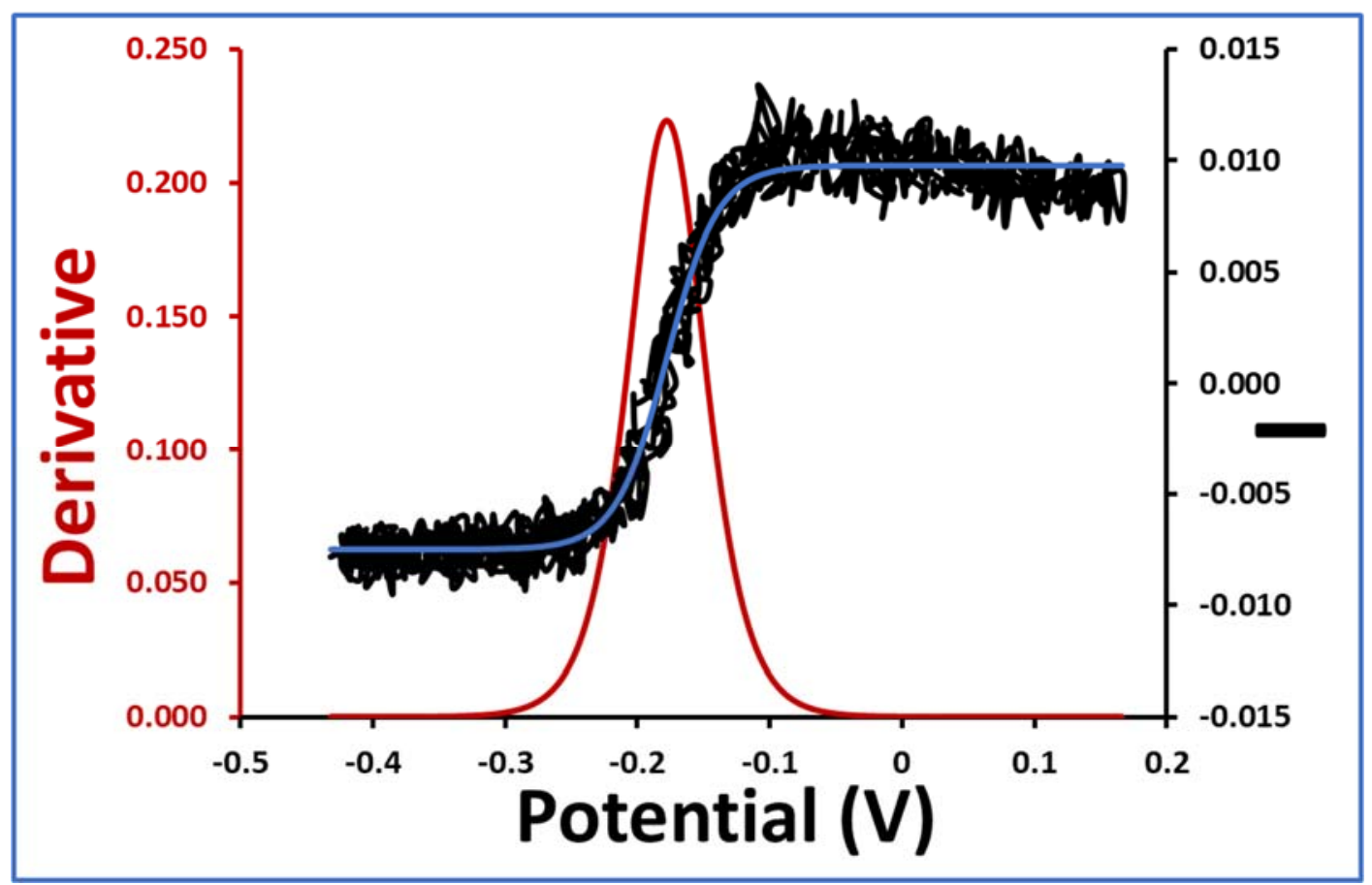

Figure 4-11: Optical signal, $I$, under a CV scan for the full immunoassay sandwich at scan rate of $0.02 \mathrm{~V} / \mathrm{s}$ (black trace). The blue trace shows the fitting of the optical signal, while the red trace represents the derivative of the fitting of the optical signal.

The red curve (y-axis on the left side of the plot) provides the derivative of the fitting of the optical signal, $I$; this trace was used to approximate the formal potential to be $-0.17 \mathrm{~V}$. After that and in order to determine the resonance frequency, a sinusoidal potential modulation was applied on the EC-SPR sensor with the full immunoassay sandwich at the 
formal potential $(-0.14 \mathrm{~V})$, and several frequencies ranging from 25 to $500 \mathrm{~Hz}$ were measured. The SPR optical signal, $\triangle I_{S P R}$, was determined by subtracting the baseline SPR optical signal, which was taken after the capture of the HA virus antigen from the SPR optical signal collected after the incubation (and rinsing) of the MB-labeled polyclonal Ab. The SPR optical signal, $\triangle I S P R$, versus the frequency of the sinusoidal potential modulation is shown in Figure 4-12.

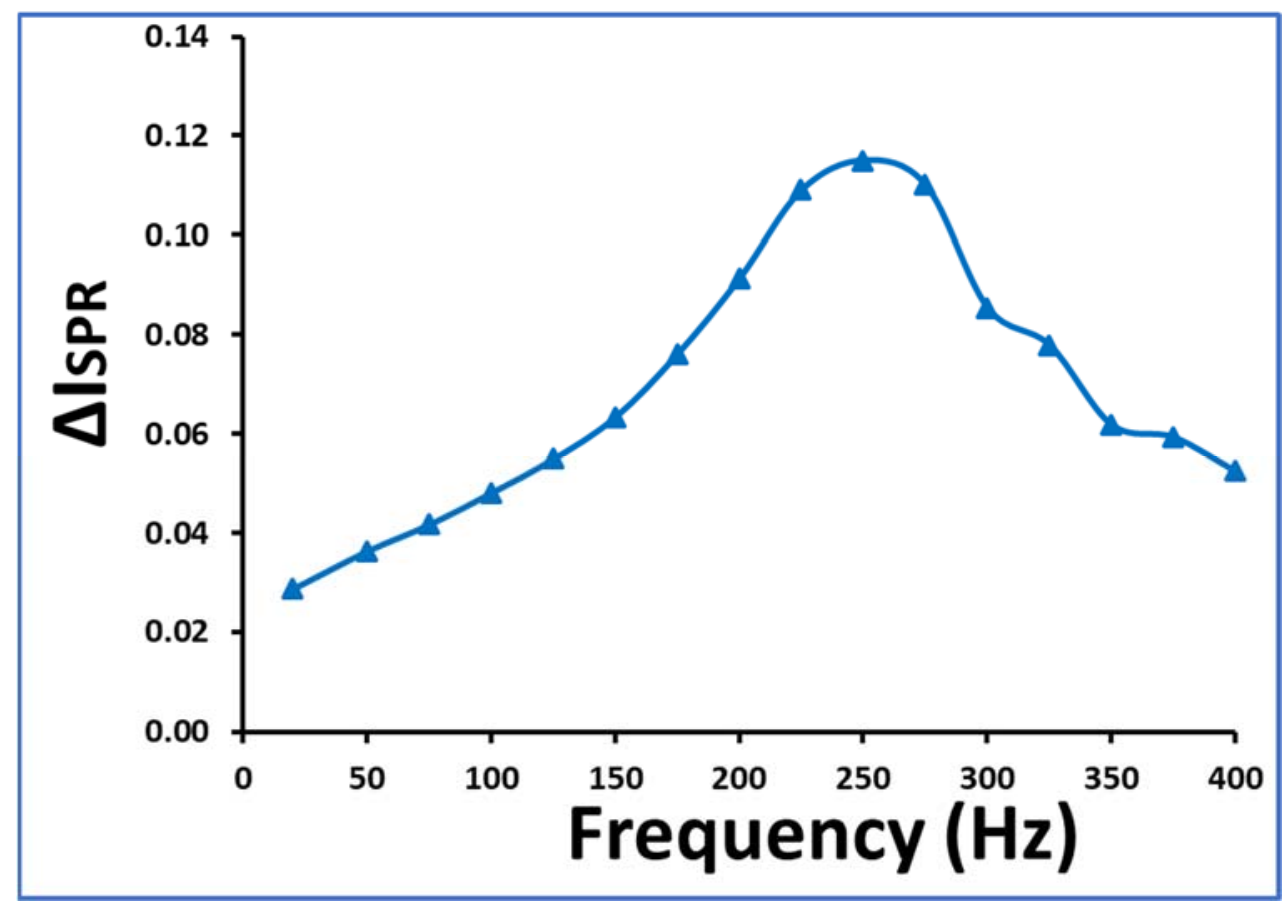

Figure 4-12: SPR optical signal, $\triangle \mathrm{I}_{\mathrm{SPR}}$, measured under AC potential modulation with the full immunoassay sandwich at the DC bias potential of $-0.14 \mathrm{~V}$ and a HA virus antigen concentration of $200 \mathrm{ng} / \mathrm{ml}$.

The results displayed in Figure 4-12 show a characteristic resonance frequency for the faradaic process with a clear peak centered at about $250 \mathrm{~Hz}$, corresponding to the highest electron transfer rate of the relevant redox process. Once the modulation frequency for the two experiments, that maximizes the SPR optical signal, $\triangle I S P R$, were determined, a 
sinusoidal potential modulation with an amplitude of $0.04 \mathrm{~V}$ and several different DC bias potentials ranging from -0.45 to $0 \mathrm{~V}$ were applied. Figure $4-13$ shows the SPR optical signal, $\triangle I_{S P R}$, obtained by the lock-in amplifier at each bias potential for (a) the full immunoassay sandwich, and (b) the MB-labeled polyclonal Ab adsorbed onto the activated MPA.

(a)

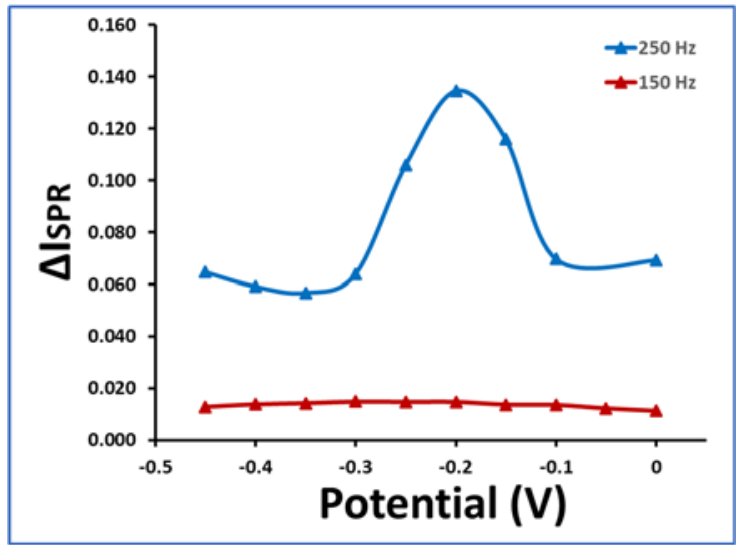

(b)

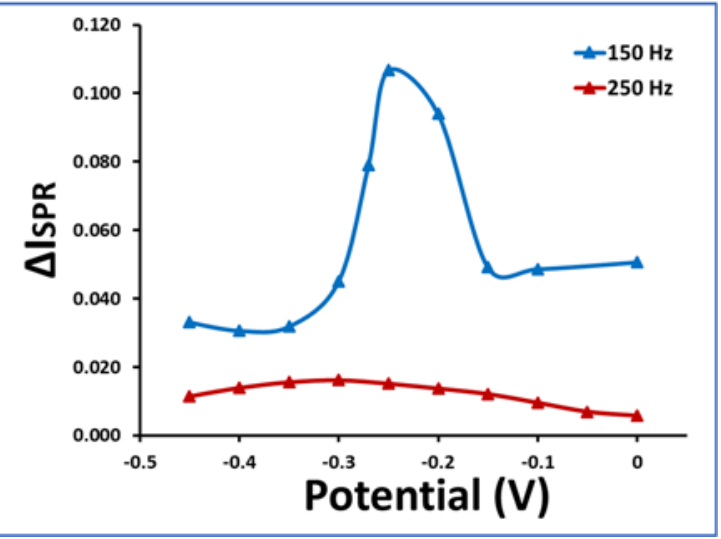

Figure 4-13: SPR optical signal, $\triangle \mathrm{I}$ SPR, versus the DC bias potential with (a) the full immunoassay sandwich, (b) the MB-labeled polyclonal Ab adsorbed onto the MPA measured under a sinusoidal potential modulation $(0.04 \mathrm{~V})$.

As shown in Figure 4-13 (a) and (b), the plot of SPR optical signal against the DC bias potential at the resonanse frequency (blue curves) displays a maximum intensity at approximately the formal potential, which confirms the redox activity of the MB probe. On the other hand, far from the resonance frequency (red curves), the SPR optical signal against the DC bias potential display almost constant optical signal. These results show the strong effects of the resonance frequency on the optical signal, $\triangle I S P R$, which is related to the sensitivity of our sensor. Thus, we expect with driving our sensor at the resonance frequency with the full immunoassay sandwich $(250 \mathrm{~Hz})$ will help to increase the optical 
signal, $\triangle I_{S P R}$, and reduce the optical signal generated by the non-specific adsorption. Moreover, this high modulation frequency will help eliminate spurious effects coming from thermal noise, mechanical vibration and other detrimental sources.

\subsubsection{Limit of Detection Measurement}

Three different concentrations of HA virus antigen $(20,50,100 \mathrm{ng} / \mathrm{ml})$ were used to determine the limit of detection. A sinusoidal potential modulation with an amplitude of $0.04 \mathrm{~V}$ and a frequency of $250 \mathrm{~Hz}$ was employed to the sensor surface. Several different DC bias potentials, ranging from -0.4 to $0 \mathrm{~V}$, were chosen to encompass the formal potential of the MB redox probe. Figure 4-14 shows the SPR optical signal, $\triangle I_{S P R}$, obtained by the lock-in amplifier at each bias potential in the absence of HA virus antigen and for the three different concentrations of HA virus antigen.

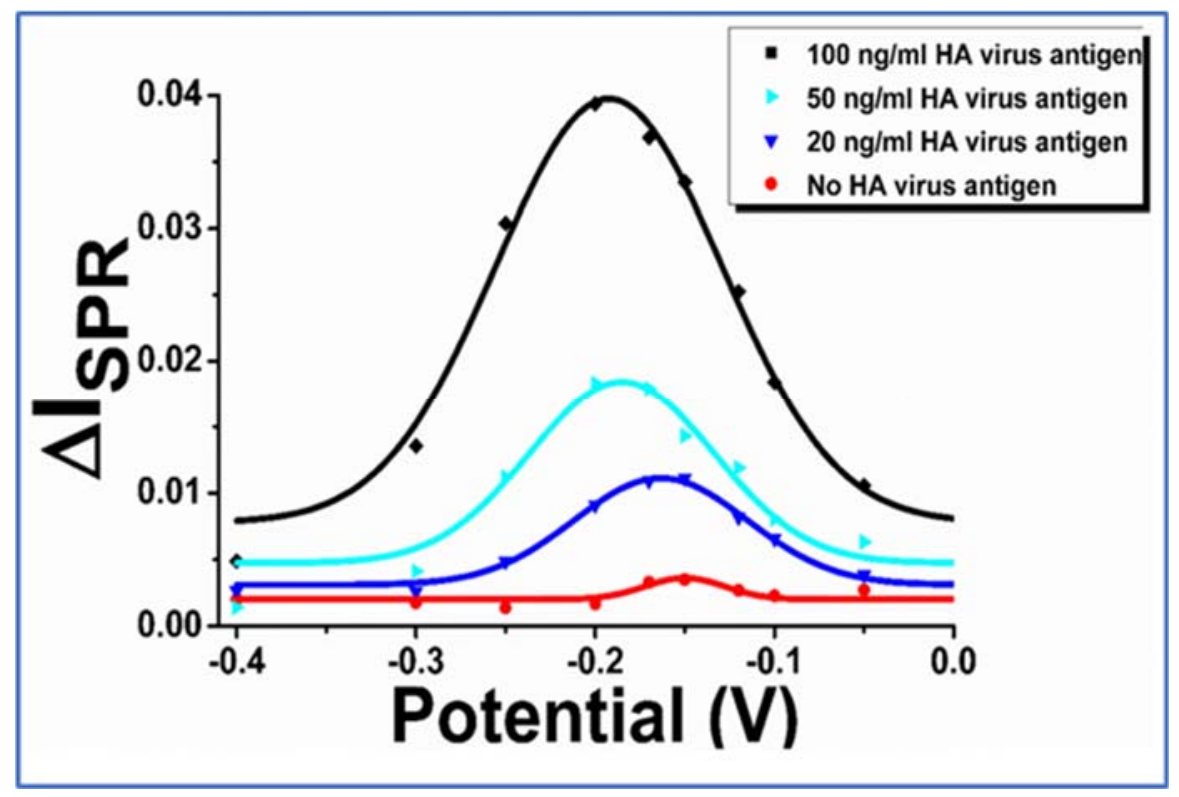

Figure 4-14: SPR optical signal, $\triangle I_{S P R}$, versus the DC bias potential in the absence of HA virus antigen and for different concentrations of the HA virus antigen measured under a 
sinusoidal potential modulation at the frequency of $250 \mathrm{~Hz}$ and an amplitude modulation of $0.04 \mathrm{~V}$.

As shown in Figure 4-14, the plot of SPR optical signal against the DC bias potential displays a maximum intensity at approximately $-0.15 \mathrm{~V}$, which confirms the redox activity of the MB probe. Away from the formal potential of the redox probe, the optical signal decreases substantially and reaches a non-zero value due to changes in the real part of the refractive index as the MB-labeled polyclonal antibody adsorbs onto the sensor surface. The peak intensity of the SPR optical signal as reported by the redox probe is proportional to the surface concentration of the HA virus antigen and provides a direct route for the quantification of the HA virus antigen in the solution. A small peak of the SPR signal in the absence of the HA virus antigen (red line in Figure 4-14) indicates the presence of a small optical signal of non-specific adsorption events. The corresponding peak intensity of the SPR optical signal for the different bulk concentrations of the HA virus antigen were determined, and the results are plotted in Figure 4-15.

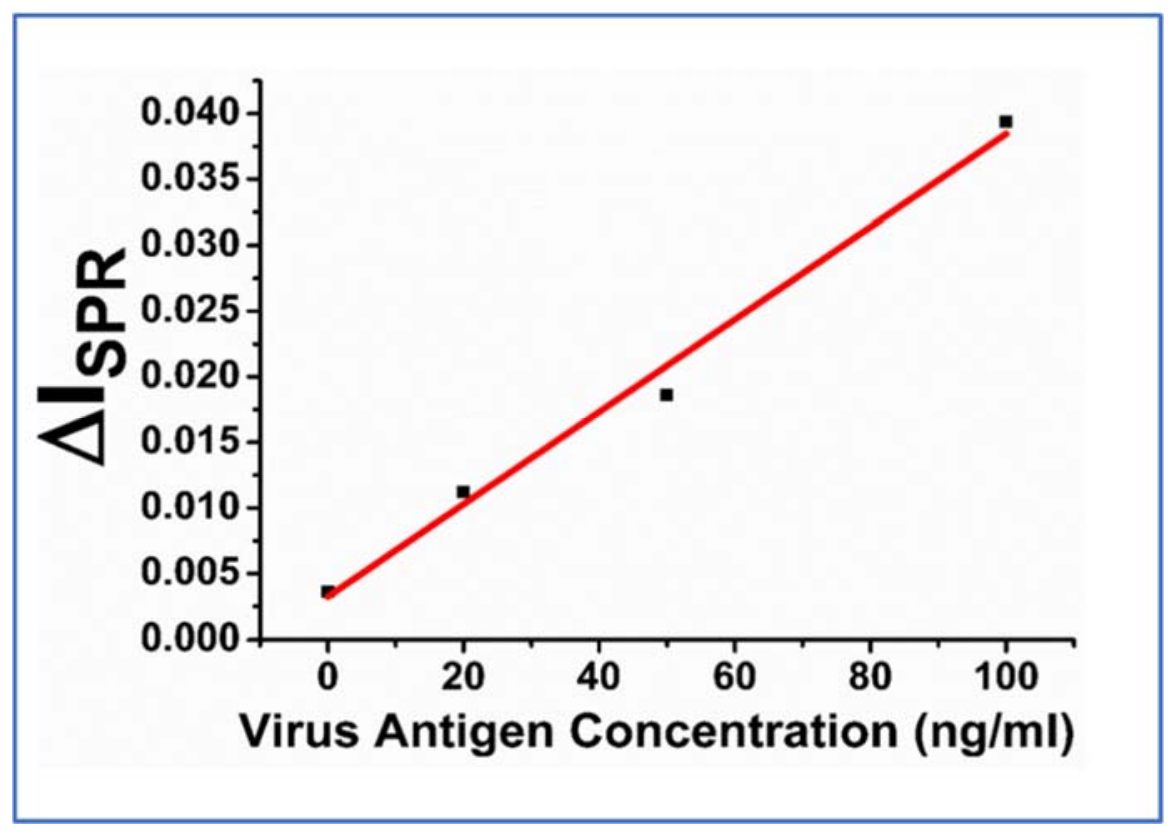


Figure 4-15: Calibration curve based on AC measurements for detection of different concentrations of the HA virus antigen.

The calibration curve for detection shows a linear response, and the limit of detection was determined using the 3-sigma standard, which corresponds to an analyte concentration that would generate a signal that is three-fold the signal measured when no virus analyte was present in the system. Such a procedure resulted in a value of $16 \mathrm{ng} / \mathrm{ml}$ or $300 \mathrm{pM}$ for the limit of detection. The detection sensitivity obtained here for the influenza virus based on an immunoassay on the EC-SPR platform shows improved performance when compared to previous work based on a redox nanoswitch probe coupled to a DNA strand (5 nM) [9]. Most importantly, the EC-SPR sensor, which features a single-bounce reflection interrogation scheme, offers a high compatibility for multiple-array detection in a small footprint that can make it a very attractive sensing technology.

\subsection{Conclusions}

In this study, an electrochemical surface plasmon resonance (EC-SPR) strategy for immunosensing detection was developed. The principle of the detection is based on a sandwich assay that deploys an electrically modulated optical signal featured by a redox probe where no chemical labeling of the analyte is required. The sensor principle takes advantage of the highly specific interaction between a monoclonal capture antibody, a virus antigen and a redox labeled antibody. The potential modulation of the redox probe creates a robust and highly selective analytical signal for specific detection of events of interest. The capabilities of the EC-SPR sensor for detection and quantification of the hemagglutinin protein from the $\mathrm{H} 5 \mathrm{~N} 1$ avian influenza A virus was demonstrated with a $300 \mathrm{pM}$ limit of detection. Overall, the EC-SPR sensor strategy has the potential for high 
sensitivity detection of multiplexed targets with a small footprint that only requires a small amount of sample material at low concentrations of a target antigen. The sensor can be further functionalized and used in clinical settings as a direct and fast quantification for detection a variety of pathogens. 


\section{CHAPTER 5}

\section{DETECTING ENDOTOXIN BACTERIA IN BIOLOGICAL SAMPLES}

\subsection{Introduction}

The electrochemically-modulated SPR platform presented in chapter 4 successfully demonstrated a novel immunosensor-based strategy for direct detection of an influenza virus prepared in PBS buffer solution. A remarkable limit of detection for an influenza virus prepared in PBS buffer solution when using a highly selective probe that is biologically specific was achieved. However, the high performance and widely applicable for detection of range of causative agents of human pathogens in complex samples such as blood are very crucial for early diagnosis and intervention in controlling the infection. Therefore, for the capability of diagnosing more difficult cases, a small-volume blood sensor can be very helpful for efficient blood sample detection in medical applications. In this chapter, the performance of the EC-SPR platform to demonstrate the competency for detecting of a gram-negative endotoxin in PBS buffer solution, as well as in whole blood and plasma, is examined. The sensing methodology using the EC-SPR device is based on a sandwich immunoassay approach similar to the assembly described in chapter 4 , section 4.2, which starts with the functionalization of the EC-SPR platform with a capturing Ab aimed at the specific target analyte. Once the target analyte species are bound to the SPR interface, they promote the immobilization of a labeled polyclonal secondary Ab with a redox active probe. 


\subsection{Gram-Negative Endotoxin Detection with Immunoassay}

The performance of the EC-SPR platform for directly detecting of gram-negative endotoxin in PBS buffer solution as well as in whole blood and plasma was tested. The endotoxin from E. Coli DH5 alpha of gram-negative bacteria in broth provided as an inkind gift from Dr. Yoder-Himes at the University of Louisville was retrieved and quantified. Endotoxin was quantified using the LAL Chromogenic Endotoxin Quantitation kit by Thermo Scientific-Pierce. A $1.7 \mathrm{EU} / \mathrm{ml}$ of endotoxin in the PBS buffer solution was used as a stock solution for the experiments. Swine whole blood was obtained from Dr. George Pantalos at the University of Louisville as an in-kind gift, following all institutional policies regarding animal tissue research. Heparinized blood was obtained from an animal that had reached its endpoint in the original set of experiments. Under an approved tissue protocol, $10 \mathrm{ml}$ of heparinized blood was collected in a $15 \mathrm{ml}$ tube. The blood was separated into whole blood and plasma (no red, white or platelet cells). Plasma was obtained by centrifuging $5.0 \mathrm{~mL}$ of whole blood @1500 RPM for 5 minutes. The plasma was carefully pipetted out, aliquoted into $500 \mu \mathrm{l}$ and stored at $-20^{\circ} \mathrm{C}$. The whole blood aliquots stored at $4{ }^{\circ} \mathrm{C}$. A secondary lipopolysaccharides (LPS) polyclonal endotoxins Ab (antilipopolysaccharide (LPS) antibody LS-C71709, LSBio) was conjugated with the

methylene blue ester. The conjugated antibody was purified by a gel separation column by Thermo Scientific-Molecular Probes Life Technologies. Finally, a UV-Vis spectrophotography was used for quantification of conjugated $\mathrm{Ab}$. 


\subsection{Functionalization Protocol of the EC-SPR Sensor Platform for Endotoxin}

\section{Detection}

A self-assembled monolayer of MPA was used to functionalize the EC-SPR platform as described in section 4.2.2. After the MPA monolayer was formed on the sensor surface, the EDC/NHS was injected into the flow-cell to activate the carboxylic groups of the MPA. In order to demonstrate the capability of the EC-SPR sensor for detection and quantification of the endotoxin, a monoclonal anti-HA (H5N1) capture Ab was introduced onto the flow cell. The subsequent change in the reflectance was monitored as a function of time, Figure 5-1.

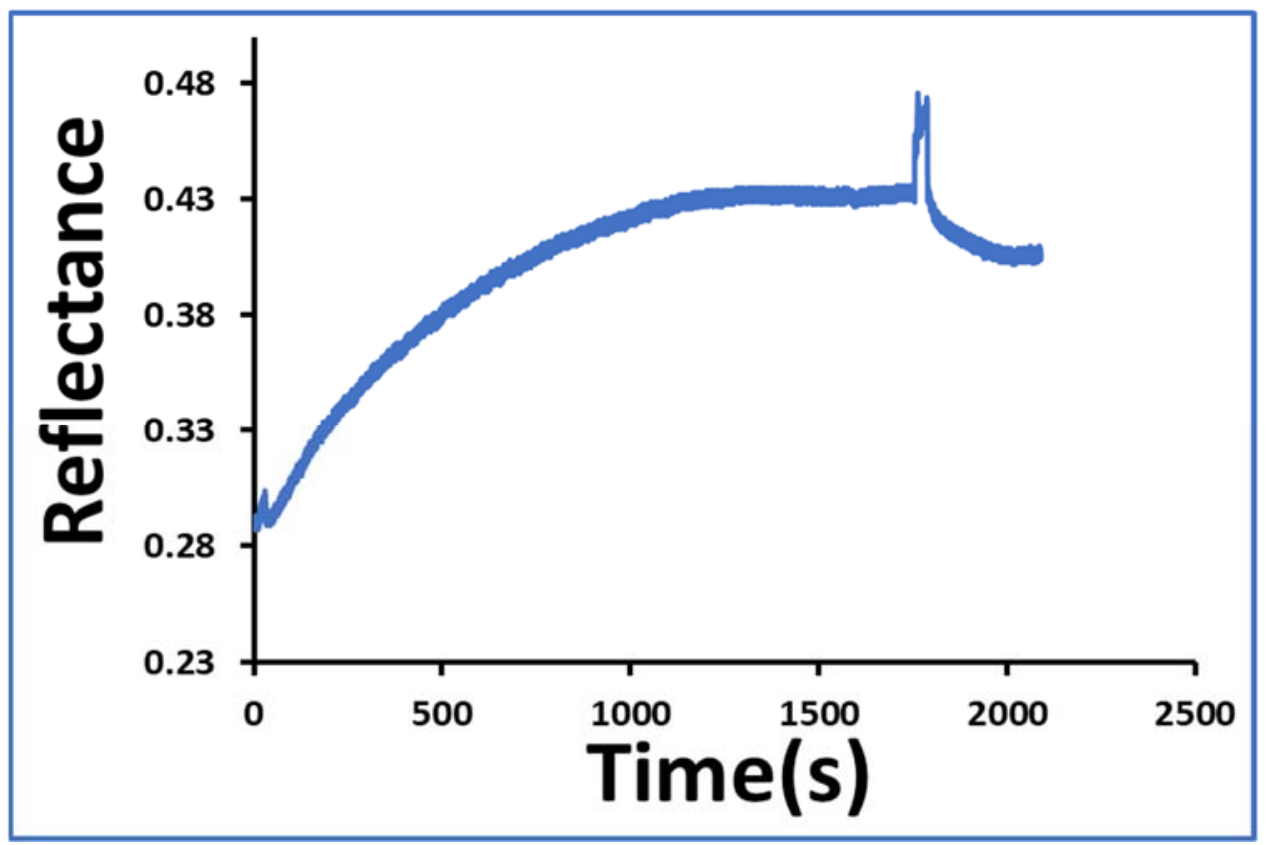

Figure 5-1: The reflectance against the time measured by the SPR technique at a fixed incident angle for a monoclonal anti-HA (H5N1) capture Ab adsorbed covalently onto MPA-modified sensor platform using EDC/NHS coupling chemistry.

Figure 5-1 shows that the monoclonal anti-HA (H5N1) capture Ab was successfully immobilized onto the sensor surface and then was reached the maximal binding capacity 
after about $1200 \mathrm{~s}$. The stable reflectance value after washing step with PBS buffer solution indicates a stable binding of the monoclonal Ab into the EC-SPR sensor platform.

\subsection{Endotoxin Detection with Immunoassay on the EC-SPR Device}

The capability of the EC-SPR sensor for the detection and quantification of endotoxin bacteria was examined. The EC-SPR sensor based on Krectchmann configuration was used here. Transverse magnetic (TM) polarized light $(660 \mathrm{~nm})$ selected by a linear retarder and a fixed linear polarizer was directed through a prism to exit the surface plasmon resonance. An electrochemical flow cell with a three-electrode configuration (working, reference, and counter electrodes) was deployed to provide an electrochemically-controlled aqueous environment in the superstrate region of the device. A power meter (Newport, Model 1930C) was deployed to collect the reflected optical signal at fixed angle. The optical signal was monitored while a potentiostat (CHI 660D, CH Instruments, Inc.) was used to control the electric potential applied to the working electrode (gold film) on the surface of the (ECSPR) device. The applied potential was referenced against a gold plated pogo pin electrode.

The detected optical signal from the power meter was sent to a lock-in amplifier (SR830 DSP, Stanford Research Sysems). A function generator (DS345, Stanford Research Systems) connected to the potentiostat provided a continuous sinusoidal wave to electrically drive the EC-SPR working electrode. An oscilloscope (Agilent, DSO8104A Infiniium) was deployed to read and record all signal traces.

\subsubsection{SPR Curves for Blood and Plasma}

As mentioned on chapter 1, the performance of the SPR sensors are highly dependent on FWHM of the resonance curve. And since the blood has different biological compositions compared to the PBS buffer solution, the resonanse angle and the width could 
have affected by the injecting of the blood in the flow cell. Therefore, in order to study this affect, the SPR reflectivity curves of PBS buffer, blood, and plasma solutions were investigated. The same setup shown in Figure 2-2, chapter 2 was employed to angular reflectance measurements. The excitation wavelength was $685 \mathrm{~nm}$. The MPA-modified gold platform was mounted in a homemade micro-electrochemical flow cell with a threeelectrode configuration. Then, the flow cell was mounted on a rotation stage to precisely control the incident angle. The SPR reflectivity curves of a PBS buffer solution was determined. Next, the blood sample was injected into the flow cell and incubated in the flow cell for 30 minutes. The flow cell was rinsed with a PBS buffer solution. After that, the SPR reflectivity curve was determined. The same procedure was used to determine the SPR reflectivity curve after incubated plasma sample in the flow cell for 30 minutes and rinse with the PBS buffer solution. Figure 5-2 shows the measured SPR reflectance curves against the effective refractive index inside the prism for the PBS buffer solution, blood, and plasma samples.

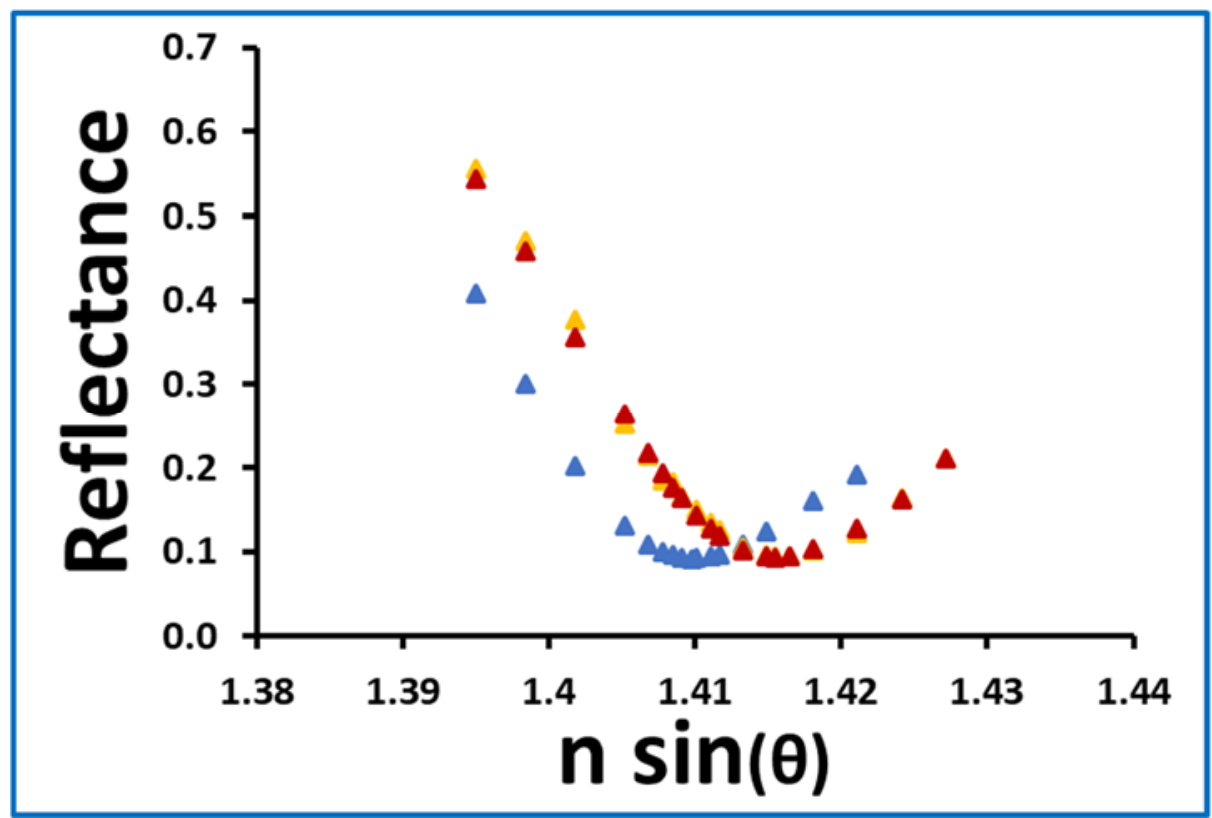


Figure 5-2: The SPR reflectance curves against the effective refractive index inside the prism for a PBS buffer solution (blue dots), plasma (yellow dots), and blood (red dots) samples.

The blue dots in Figure 4-2 shows the SPR reflectance for the PBS buffer solution while the yellow and the red dots show the SPR reflectance for plasma and blood samples, respectively. A shift in the position of the minimum reflectance (resonance angle) was observed for plasma and blood samples due to their high refractive index compared to the PBS buffer solution. Moreover, the result shows that the plasma and blood samples do not make much effect on width of SPR curves, which gives a direct indication that the EC-SPR sensor can be made possible for blood and plasma samples detection, with keeping the high sensor's accuracy and sensitivity.

\subsubsection{Specific Detection of Endotoxin in PBS Buffer Solution and the Limit of}

\section{Detection}

In this section, the performance of EC-SPR sensor for specific detection of endotoxin in PBS buffer solution is investigated. After the sensor platform was mounted on the flow cell and the monoclonal $\mathrm{Ab}$ immobilizing was confirmed, two measurements using the $\mathrm{AC}$ modulation technique are carried out. In the first measurement, a $50 \mu \mathrm{L}$ solution of MBlabeled polyclonal $\mathrm{Ab}$ at a concentration of $10 \mu \mathrm{g} / \mathrm{ml}$ was incubated in the flow cell for 30 minutes before rinsing with a PBS buffer solution. In the second measurement, a $50 \mu \mathrm{L}$ solution of $0.05 \mathrm{EU} / \mathrm{ml}$ endotoxin was prepared in $0.1 \mathrm{M}$ PBS buffer solution and incubated in the flow cell for 30 minutes before rinsing with the PBS buffer solution. This step was followed by injecting a $50 \mu \mathrm{L}$ solution of MB-labeled polyclonal Ab solution at a concentration of $10 \mu \mathrm{g} / \mathrm{ml}$ and incubating for 30 minutes before the flow cell was rinsed 
with PBS buffer solution to remove any unbound MB-labeled polyclonal antibodies. The formal potential of immobilized MB-labeled polyclonal $\mathrm{Ab}$ redox reactions with the full immunoassay sandwich was determined electrochemically using a cyclic voltammetry (CV) technique to be $-0.25 \mathrm{~V}$. Then a sinusoidal potential modulation was applied on the EC-SPR sensor platform at a fixed DC bias potential $(-0.25 \mathrm{~V})$, and several frequencies ranging from 0.1 to $1.4 \mathrm{kHz}$ were measured. Figure 5-3 shows the SPR optical signal, $\triangle I_{S P R}$, under cyclic voltammetry.

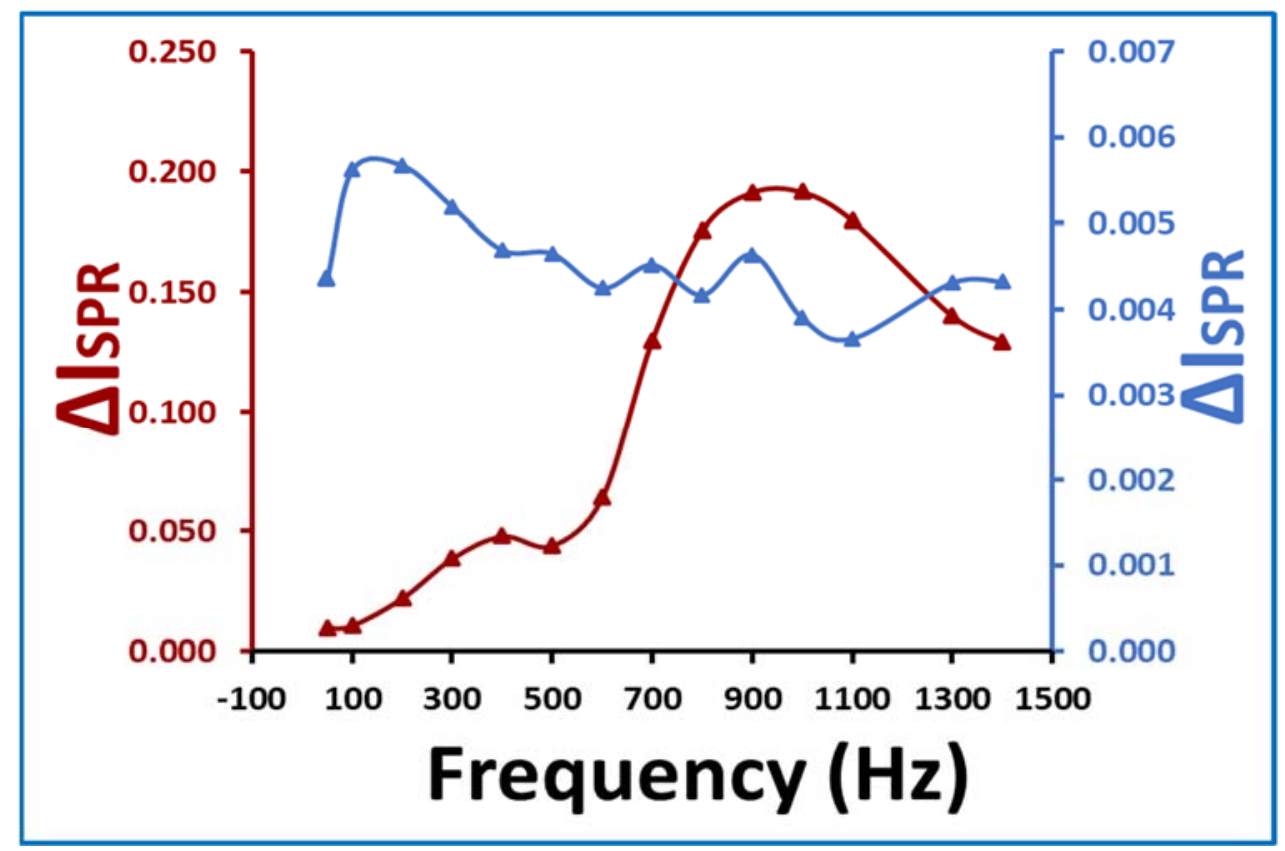

Figure 5-3: SPR optical signal, $\triangle I S P R$, measured under AC potential modulation at the DC bias potential of $-0.2 \mathrm{~V}$ in the presence of the $0.05 \mathrm{EU} / \mathrm{ml}$ endotoxin in the flow cell (red curve), and in the absence of the endotoxin in the flow cell (blue curve).

The Figure 5-3 shows a clear peak centered at about $1 \mathrm{kHz}$ which corresponds to the highest electron-transfer rate of the MB redox process was determined in the presence of the 0.05 $\mathrm{EU} / \mathrm{ml}$ endotoxin in the flow cell (red curve). On the other hand, in the absence of the endotoxin in the flow cell (blue curve), the non-specific adsorption of the MB-labeled 
polyclonal $\mathrm{Ab}$ on the device interface showed a peak at about $0.2 \mathrm{kHz}$. Then, the EC-SPR sensor was driving at the resonance frequency with the full immunoassay sandwich (1 $\mathrm{kHz}$ ). A sinusoidal potential modulation with an amplitude of $0.04 \mathrm{~V}$ was employed to the sensor surface. Several different DC bias potentials, ranging from $-0.5 \mathrm{~V}$ to $0 \mathrm{~V}$, were chosen to involve the formal potential of the MB redox probe. Figure 5-4 shows the SPR optical signal obtained by the lock-in amplifier at each bias potential in the absence of endotoxin and for a $0.05 \mathrm{EU} / \mathrm{ml}$ endotoxin in PBS buffer solution.

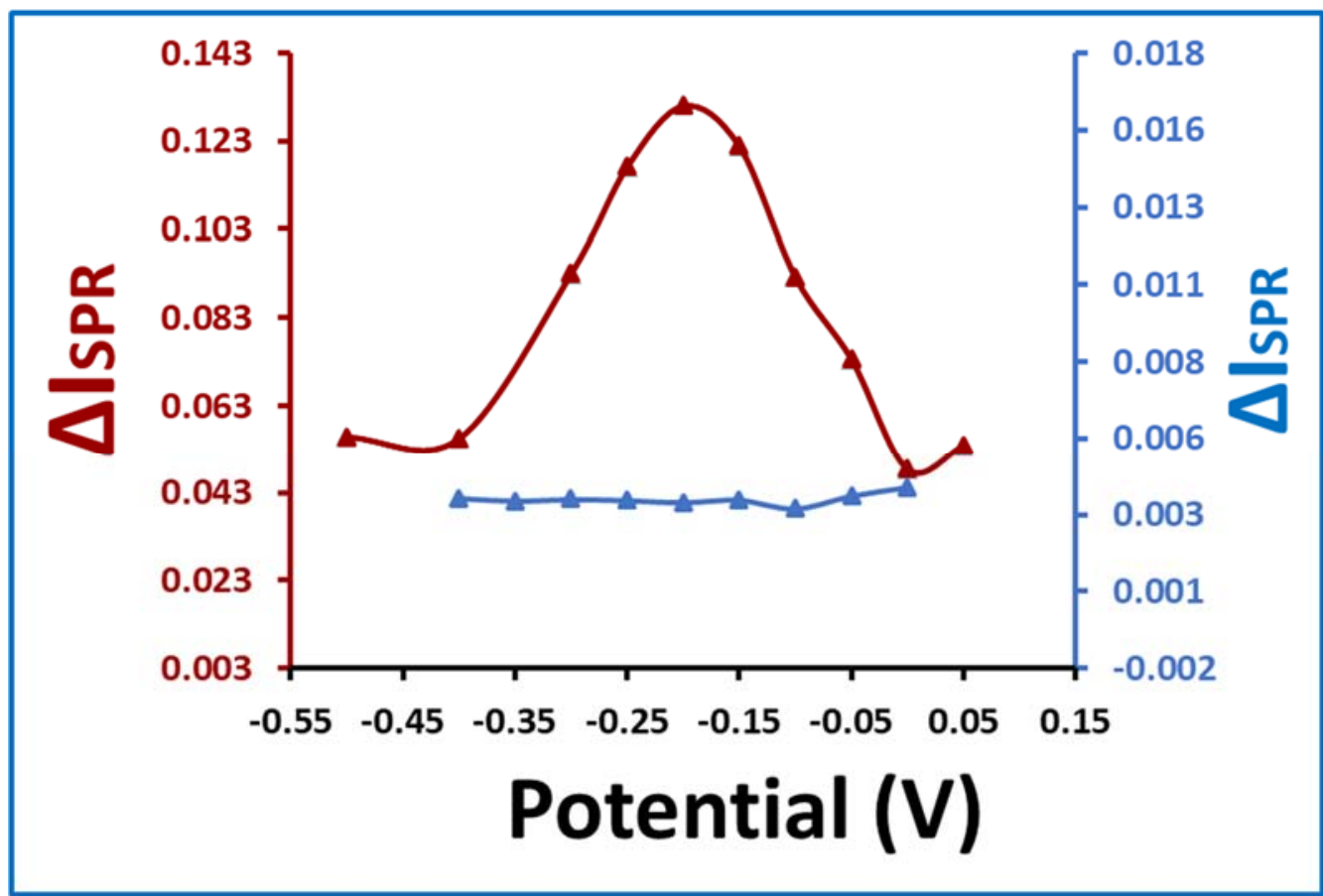

Figure 5-4: SPR optical signal, $\triangle I_{S P R}$, versus the DC bias potential in the absence of endotoxin (blue curve) and for $0.05 \mathrm{EU} / \mathrm{ml}$ endotoxin present in PBS buffer solution (red curve) measured under a sinusoidal potential modulation at the frequency of $1 \mathrm{kHz}$ and an amplitude modulation of $0.04 \mathrm{~V}$.

The results in Figure 5-4 demonstrated the capability of the EC-SPR sensor for the detection of endotoxin in PBS buffer solution under an AC voltammetry technique. 
Moreover, the non-specific adsorption of the MB-labeled polyclonal $\mathrm{Ab}$ on the device interface was successfully kept to negligible levels.

Finally, the limit of detection was measured. The same AC measurement's procedure as earlier was used with three different concentrations $(0.05 \mathrm{EU} / \mathrm{ml}, 0.06 \mathrm{EU} / \mathrm{ml}$, and 0.08 $\mathrm{EU} / \mathrm{ml}$ ). Figure 5-5 shows the SPR optical signal, $\triangle I_{S P R}$, in the absence of endotoxin and for the three concentrations of endotoxin.

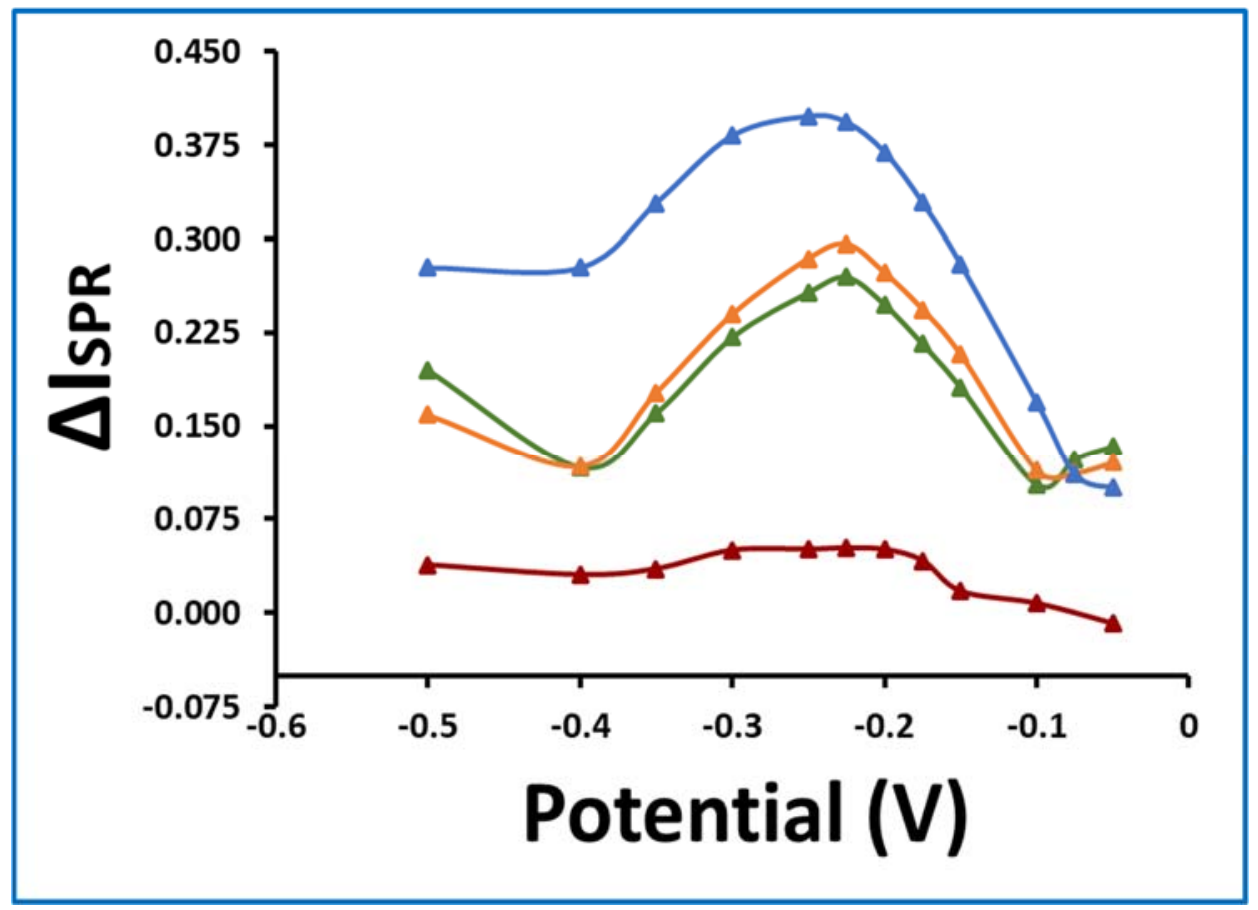

Figure 5-5: SPR optical signal, $\triangle I_{S P R}$, versus the DC bias potential in the absence of endotoxin and for different concentrations of the endotoxin measured under a sinusoidal potential modulation at the frequency of $1 \mathrm{KHz}$ and an amplitude modulation of $0.04 \mathrm{~V}$.

Three peaks at approximately $-0.25 \mathrm{~V}$ was displayed with the presence of endotoxin in the flow cell, which confirms the redox activity of the MB redox probe. The peaks intensity as reported by the redox probe is proportional to the surface concentration of the endotoxin. A small and broad peak in the absence of the endotoxin was shown (red line in Figure 5- 
5) indicates the small effect of non-specific adsorption events. The detection range of ECSPR sensor for specific detection of endotoxin in PBS buffer solution shows a linear response, Figure 5-6. A standard 3-sigma limit of detection was determined to be about $0.025 \mathrm{EU} / \mathrm{ml}$ or $2.5 \mathrm{pg} / \mathrm{ml}$.

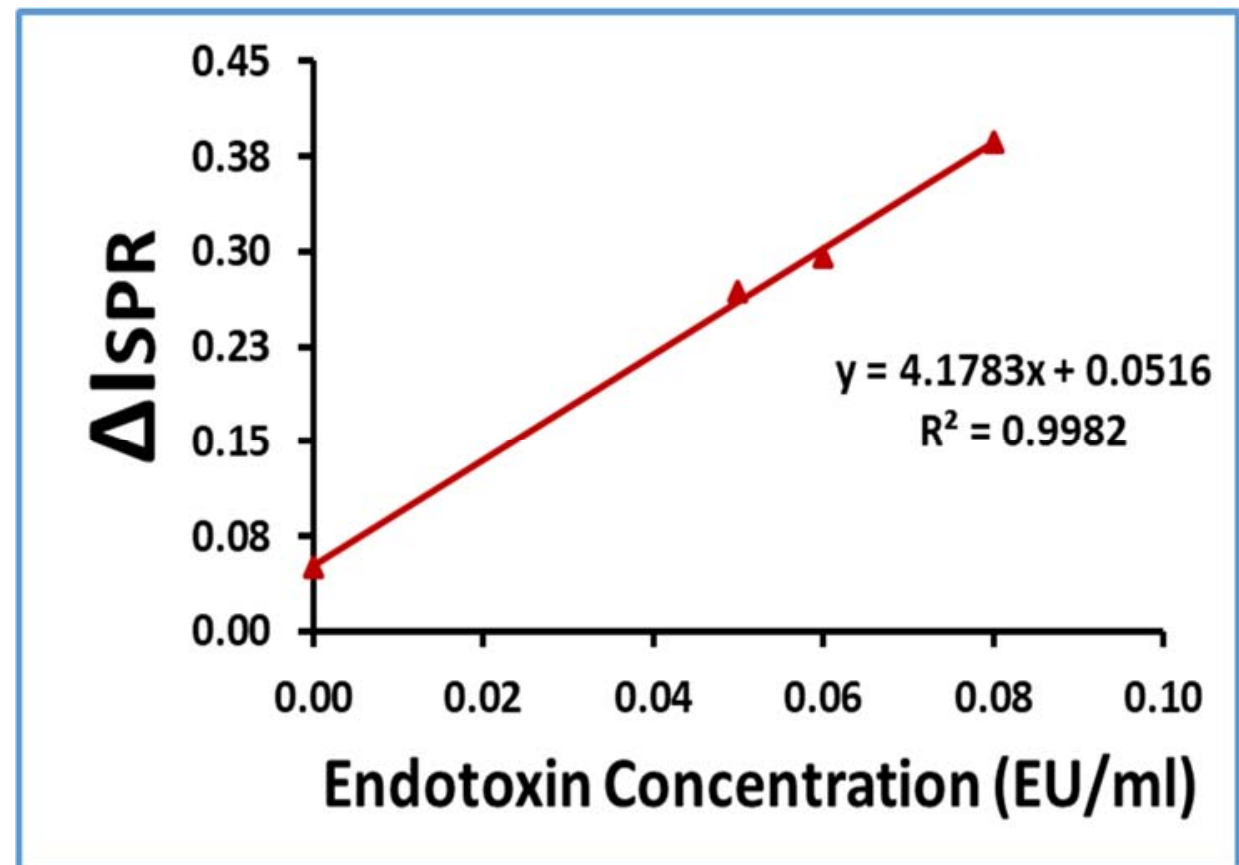

Figure 5-6: Calibration curve based on AC measurements for detection of different concentrations of the endotoxin.

Such a limit of detection already surpasses several technologies currently being deployed and places the novel strategy for specific bacteria detection with multiplexed interrogation confined to a small device footprint that can make it a very attractive sensing technology at the frontiers of the state-of-art.

\subsubsection{Specific Detection of Endotoxin in Complex Samples (Blood and Plasma)}

The performance of the EC-SPR sensor was tested directly in whole blood and plasma samples. For these tests, the surface was functionalized with the capturing 
monoclonal $\mathrm{Ab}$, and then washed with the PBS buffer solution. The same two measurements in section 5.4.2 were implemented here with endotoxin spiked in blood and plasma instead of using the endotoxin in the PBS buffer solution. Figure 5-7 shows the SPR optical signal, $\triangle I_{S P R}$, measured under AC potential modulation at a fixed DC bias potential of $0.05 \mathrm{~V}$ (measured electrically), and several frequencies ranging from 0.1 to 2 $\mathrm{kHz}$.

(a)

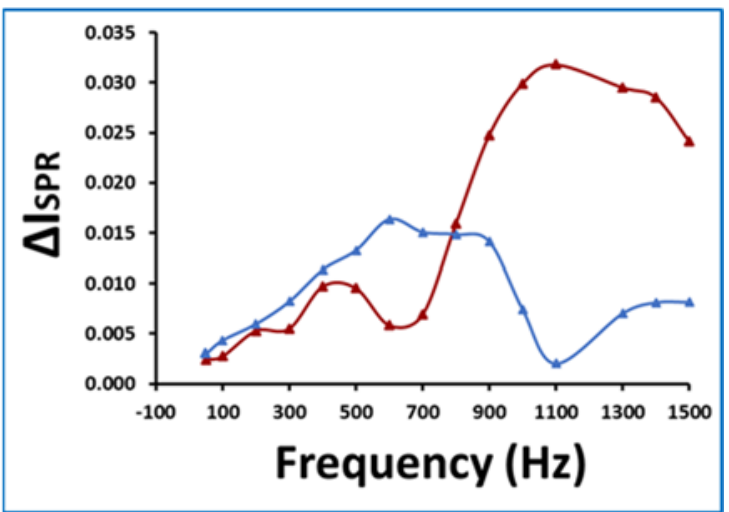

(b)

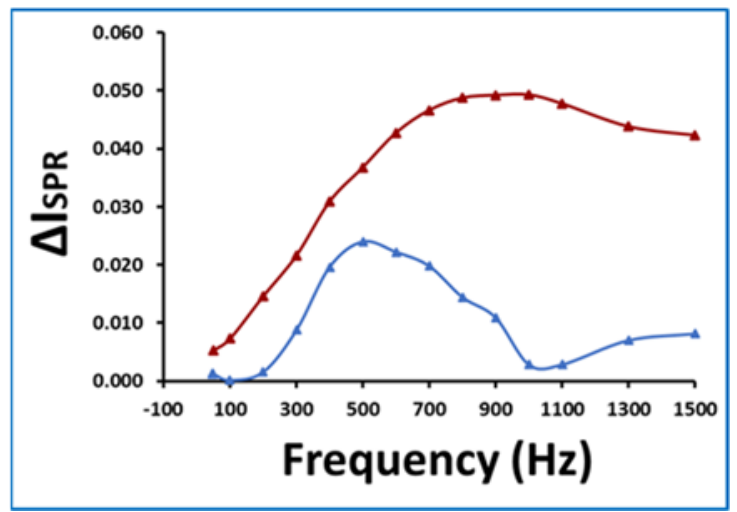

Figure 5-7: (a) SPR optical signal, $\triangle I_{S P R}$, measured under AC potential modulation at the DC bias potential of $-0.05 \mathrm{~V}$, red curve with $0.05 \mathrm{EU} / \mathrm{ml}$ endotoxin spiked on blood, blue curve without endotoxin. (b) SPR optical signal, $\triangle I_{S P R}$, versus the DC bias potential, red curve with $0.05 \mathrm{EU} / \mathrm{ml}$ endotoxin spiked on plasma, blue curve without Endotoxin.

The red curve (Figure 5-7(a)) with $0.05 \mathrm{EU} / \mathrm{ml}$ endotoxin spiked in blood shows a clear peak at about $1 \mathrm{kHz}$ corresponding to the $\mathrm{MB}$ redox process which indicates the presence of the endotoxin, while the blue curve without the presence endotoxin in the flow cell shows a peak at about $500 \mathrm{~Hz}$ which is related to the non-specific adsorption. The same results were obtained with the presence of the endotoxin spiked in plasma, Figure 5-7(b). Then, a frequency of $1 \mathrm{kHz}$ was used to apply the AC voltammetry technique. A sinusoidal 
potential modulation with an amplitude of $0.04 \mathrm{~V}$ was employed with a DC bias potential that varied over the range of $-0.3 \mathrm{~V}$ to $0.3 \mathrm{~V}$ for encompassing the formal potential of the redox process of the MB redox probe, Figure 5-8.

(a)

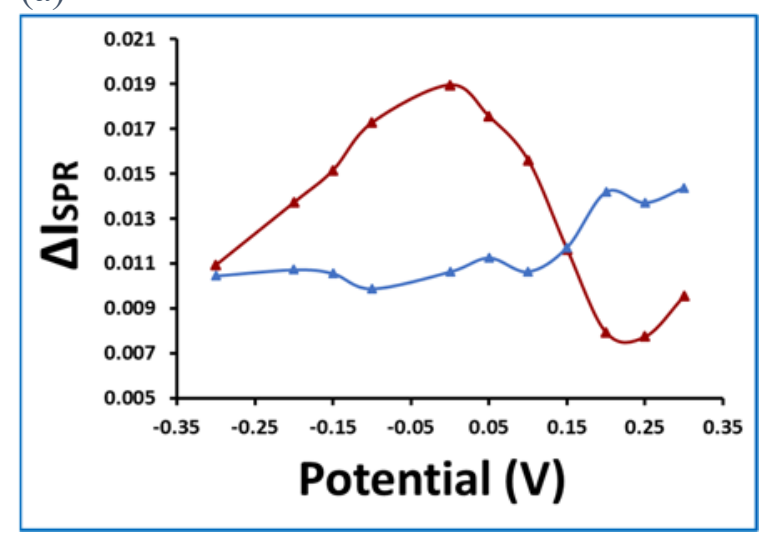

(b)

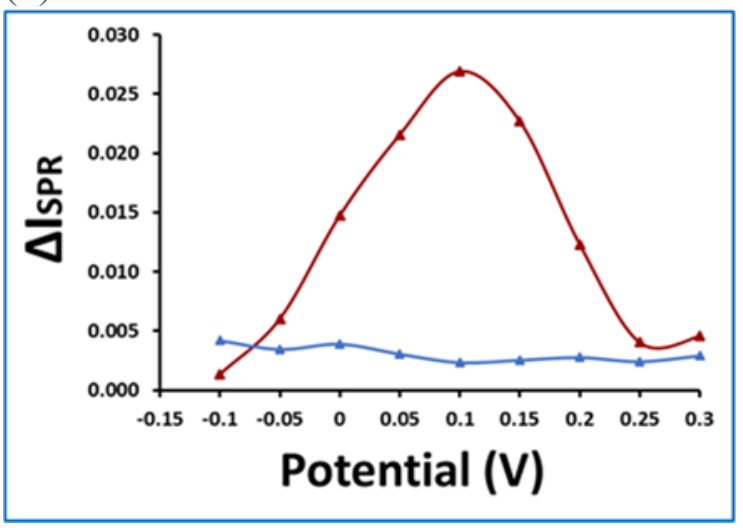

Figure 5-8: SPR optical signal, $\triangle I_{S P R}$, versus the DC bias potential, (a) red curve with

$0.05 \mathrm{EU} / \mathrm{ml}$ endotoxin spiked in blood, blue curve without endotoxin, (b) red curve with $0.05 \mathrm{EU} / \mathrm{ml}$ endotoxin spiked in plasma, blue curve without Endotoxin.

As shown in Figure 5-8 (a) and (b), peaks reported by the redox probe (red curves) provide a clear and direct route to the detection of the specific endotoxin in whole blood and plasma. Moreover, these results indicate that driving the EC-SPR sensor in AC voltammetric modulation can eliminate the effect of the optical signal of the non-specific adsorption (blue curves).

\subsection{Conclusion}

In this study, the performance of the EC-SPR platform for detecting and identification of causative agents of human pathogens was demonstrated. Biological samples from Heparinized blood spiked with gram-negative bacteria in broth were tested to demonstrate the competence of detection. The experimental results have successfully demonstrated the 
detection and identify the presence of gram-negative bacteria in complex samples (whole blood and plasma) with outstanding limits of detection in the pico-molar range. Based on these results, we believe that our sensor can use in clinical settings as a fast and sensitive way to diagnose the most common pathogens like bacteria, fungi, and virus. 


\section{CHAPTER 6}

\section{SUMMARY AND OUTLOOKS}

\subsection{Summary}

A novel electrochemical surface plasmon resonance (EC-SPR) technology was developed for challenging investigations and applications in different redox assemblies. The EC-SPR methodology based on the detection of an optical output signal difference between the reduced and oxidized states of a redox-active probe after an electrochemically modulates the noble-metal surface of an SPR platform. Characterization and optimization of the EC-SPR device to perform a superior performance and successfully adapted for spectroelectrochemical measurements were experimentally demonstrated. The combination between the EC-SPR device and the optical impedance spectroscopy technique (OIS) experimentally demonstrated a new path to detect fast reaction rate constants of a surface-adsorbed redox species. The new methodology was employed for studying the impact of different functionalizing layers and immobilization strategies on the electron-transfer rate of cytochrome c protein that were adsorbed on the platform.

Using the EC-SPR device a new strategy for immunosensing detection based on a highly specific interaction between a monoclonal capture antibody, a virus antigen and a redox labeled antibody was developed. The potential modulation of the redox probe creates a robust and highly selective analytical signal for specific detection of events of interest. 
The capability of the new strategy for the detection and quantification of a hemagglutinin (HA) protein from the $\mathrm{H} 5 \mathrm{~N} 1$ avian influenza A virus in a PBS buffer solution was demonstrated. Moreover, the capability of the EC-SPR device to directly detect and reproducibly identify the presence of gram-negative bacteria in complex samples (whole blood and plasma) was successfully demonstrated. The experimental results with viral and endotoxin biomaterials have produced outstanding limits of detection in the pico-molar range.

In conclusion, it can be stated that the EC-SPR technology provides a cost-effective, small-size, and simple transduction platform that offers a reliable path for investigating a variety of redox-transduced events with extremely low surface densities. Moreover, the EC-SPR sensor strategy has the potential for high sensitivity detection of multiplexed targets, that can open up new ways, in clinical settings, for a fast and sensitive diagnosis of the most common pathogens.

\subsection{Outlook}

\subsubsection{Nanostructured Semiconductors Investigations}

The EC-SPR device has demonstrated the ability to create the opportunity to employ very small electrochemical cells enabling measurements at much higher modulation frequencies to detect fast reaction rate constants. Using these advantages, the EC-SPR could be applied to investigate semiconductor nanomaterials shaped as nanocrystals and nanorods. These types of semiconductor nanomaterials have generated significant interest as potential photocatalytic materials for various reactions. Determination of the energetics of those nanomaterials is a critical first step to the design of photovoltaic devices with superior photocatalytic performance. Furthermore, these nanomaterials are ideal model 
systems to study electrochemical processes, as the size, composition, and morphology of these materials can be precisely controlled to dictate charge-transfer processes. Studying these semiconductor colloids will benefit from the implementation of the EC-SPR device at much high modulation frequencies. However, in order to characterize the nanomaterials described above, a device with electrode material to obtain broader spectral range, higher modulation frequencies, versatility for different surface chemistries, and tunability of potential window is greatly needed. For example, there exists a unique opportunity to study metal-tipped semiconductor nanorods which are anticipated to exhibit relatively fast charge transfer kinetics (on the electrochemical timescale), but their characterization has been problematic so far by the fairly weak spectroscopic features they display. A gold-tipped CdSe nanorod exhibits weak excitonic spectral features as a consequence of the noble metal inclusions and is anticipated (but have not been measured yet) to exhibit rapid electron transfer as globally observed in photocatalysis reactions $[157,158]$. Our unique EC-SPR spectroscopy technique will allow us to systematically characterize nanocolloidnanocolloid interactions, and apparent charge transfer rate constants as a function of surface coverage.

\subsubsection{Fluorescence- Based EC-SPR Immunosensor}

Fluorescence-based biosensors are considered a very high sensitivity technique for detecting low concentrations of biomolecules. The total internal reflection fluorescence (TIRF)[159, 160], the supercritical angle fluorescence method (SAF)[161-163], and the surface plasmon-coupled emission (SPCE)[164-166] are widely used for detection of very low concentrations of analytes due to their high sensitivity. Despite the high sensitivity and surface selectivity of these techniques, the fluorescing background from biological 
components present in the bulk phase and the optical components can impair the performance of these techniques-based biosensors. However, for developing a novel immunosensor-based strategy for direct detection of human pathogens with high sensitivity and less background signal, an incorporating a sandwich bioassay on an electrochemically modulated SPR fluorescent can be advantageous. We hypothesize that this new technology will lead to an additional breakthrough in EC-SPR sensor since the fluorescent signal grows from negligible background, the SPR enhance the electromagnetic field for the excitation of the fluorophores, and the semitransparent metal film acts as an efficient blocker to reduce the background contribution from the excitation light source. More important, both the potential modulation and the optical excitation through the SPR platform will be driving mechanisms of the analytical signal that are strictly confined to the device surface, which will help to reduce the background signals. Superior performance with potential-modulated fluorescent probes is envisioned to I) lead to limits of detection that will at least match those from ELISA and PCR, and II) will establish a new technology that offers substantially shorter detection times, more cost-effective protocols, and simpler operating procedures, which are all critical characteristics needed for point-of-care applications. 


\section{REFERENCES}

1. Thevenot, P., W. Hu, and L. Tang, Surface chemistry influences implant biocompatibility. Current topics in medicinal chemistry, 2008. 8(4): p. 270-280.

2. Wang, H., Y. Yang, and L. Guo, Renewable-Biomolecule-Based Electrochemical EnergyStorage Materials. Advanced Energy Materials, 2017. 7(23): p. 1700663.

3. Schmidt, W.G., et al., Organic molecule adsorption on solid surfaces: chemical bonding, mutual polarisation and dispersion interaction. Applied Physics A, 2006. 85(4): p. 387397.

4. Tanaka, K., N. Okui, and T. Sakai, Molecular orientation behavior of paraffin thin films made by vapor deposition. Thin Solid Films, 1991. 196(1): p. 137-145.

5. Kowarik, S., A. Gerlach, and F. Schreiber, Organic molecular beam deposition: fundamentals, growth dynamics, andin situstudies. Journal of Physics: Condensed Matter, 2008. 20(18): p. 184005.

6. Abrishami, M., et al., PCR detection and identification of bacterial contaminants in ocular samples from post-operative endophthalmitis. Journal of clinical and diagnostic research : JCDR, 2015. 9(4): p. NC01-NC3.

7. Yamamoto, Y., PCR in diagnosis of infection: detection of bacteria in cerebrospinal fluids. Clinical and diagnostic laboratory immunology, 2002. 9(3): p. 508-514.

8. Ben-Amar, A., S. Oueslati, and A. Mliki, Universal direct PCR amplification system: $a$ time- and cost-effective tool for high-throughput applications. 3 Biotech, 2017. 7(4): p. 246-246.

9. Choi, S. and J. Chae, Reusable biosensors via in situ electrochemical surface regeneration in microfluidic applications. Biosens Bioelectron, 2009. 25(2): p. 527-31.

10. Chen, Y., et al., Recent advances in rapid pathogen detection method based on biosensors. European Journal of Clinical Microbiology \& Infectious Diseases, 2018. 37(6): p. 1021-1037.

11. Mohanty, S.P. and E. Kougianos, Biosensors: a tutorial review. IEEE Potentials, 2006. 25(2): p. 35-40.

12. Engel, A., C.-A. Schoenenberger, and D.J. Müller, High resolution imaging of native biological sample surfaces using scanning probe microscopy. Current Opinion in Structural Biology, 1997. 7(2): p. 279-284.

13. You, H.X. and C.R. Lowe, Progress in the application of scanning probe microscopy to biology. Current Opinion in Biotechnology, 1996. 7(1): p. 78-84.

14. Garrison, M.D. and B.D. Ratner, Scanning probe microscopy for the characterization of biomaterials and biological interactions. Ann N Y Acad Sci, 1997. 831: p. 101-13.

15. Leggett, G.J., et al., Scanning probe microscopy of biomolecules and polymeric biomaterials. Journal of Electron Spectroscopy and Related Phenomena, 1996. 81(3): p. 249-268.

16. Duncan, W.M. and S.A. Henck, Insitu spectral ellipsometry for real-time measurement and control. Applied Surface Science, 1993. 63(1): p. 9-16.

17. Plekhanov, A.I. and V.V. Shelkovnikov, Optical constants of nanofilms of J aggregates of organic dyes, measured by spectral ellipsometry and polarization reflectometry. Optics and Spectroscopy, 2008. 104(4): p. 545.

18. Brecht, A., J. Ingenhoff, and G. Gauglitz, Direct monitoring of antigen-antibody interactions by spectral interferometry. Sensors and Actuators B: Chemical, 1992. 6(1): p. 96-100. 
19. Brecht, A., G. Gauglitz, and J. Polster, Interferometric immunoassay in a FIA-system: a sensitive and rapid approach in label-free immunosensing. Biosensors and Bioelectronics, 1993. 8(7): p. 387-392.

20. Striebel, C., A. Brecht, and G. Gauglitz, Characterization of biomembranes by spectral ellipsometry, surface plasmon resonance and interferometry with regard to biosensor application. Biosensors and Bioelectronics, 1994. 9(2): p. 139-146.

21. Blidar, A., et al., Electrochemical surface plasmon resonance (EC-SPR) aptasensor for ampicillin detection. Analytical and Bioanalytical Chemistry, 2019. 411(5): p. 1053-1065.

22. Bond, A.M., Broadening electrochemical horizons : principles and illustration of voltammetric and related techniques. Oxford science publications. 2002, Oxford ;: Oxford University Press.

23. Salam, F. and I.E. Tothill, Detection of Salmonella typhimurium using an electrochemical immunosensor. Biosensors and Bioelectronics, 2009. 24(8): p. 2630-2636.

24. Mao, K., et al., Label-free electrochemical immunosensor based on graphene/methylene blue nanocomposite. Analytical Biochemistry, 2012. 422(1): p. 22-27.

25. $\mathrm{Li}, \mathrm{H}$., et al., Electrochemical immunosensor with $\mathrm{N}$-doped graphene-modified electrode for label-free detection of the breast cancer biomarker CA 15-3. Biosensors and Bioelectronics, 2013. 43: p. 25-29.

26. Wang, H., et al., Label-free electrochemical immunosensor for prostate-specific antigen based on silver hybridized mesoporous silica nanoparticles. Analytical Biochemistry, 2013. 434(1): p. 123-127.

27. Li, N., et al., Disposable Immunochips for the Detection of Legionella pneumophila Using Electrochemical Impedance Spectroscopy. Analytical Chemistry, 2012. 84(8): p. 34853488.

28. Kozhevnikov, E., et al., Electrical impedance spectroscopy-a potential method for the study and monitoring of a bone critical-size defect healing process treated with bone tissue engineering and regenerative medicine approaches. Journal of Materials Chemistry B, 2016. 4(16): p. 2757-2767.

29. Dean, D.A., et al., Electrical Impedance Spectroscopy Study of Biological Tissues. Journal of electrostatics, 2008. 66(3-4): p. 165-177.

30. Huang, Y., et al., Disease-Related Detection with Electrochemical Biosensors: A Review. Sensors (Basel, Switzerland), 2017. 17(10): p. 2375.

31. Florea, A., et al., Electrochemical Biosensors as Potential Diagnostic Devices for Autoimmune Diseases. Biosensors, 2019. 9(1): p. 38.

32. Cohen, D.J., B.C. King, and F.M. Hawkridge, Spectroelectrochemical and electrochemical determination of ligand binding and electron transfer properties of myoglobin, cyanomyoglobin, and imidazolemyoglobin. Journal of Electroanalytical Chemistry, 1998. 447(1): p. 53-62.

33. Keyes, T.E. and R.J. Forster, 14 - Spectroelectrochemistry, in Handbook of Electrochemistry, C.G. Zoski, Editor. 2007, Elsevier: Amsterdam. p. 591-635.

34. Sagara, T., et al., Voltammetric application of electromodulated electroreflection absorption spectroscopy: electroreflectance voltammetry as an in situ spectroelectrochemical technique. Langmuir, 1991. 7(5): p. 1005-1012.

35. Feng, Z.Q., T. Sagara, and K. Niki, Application of potential-modulated UV-visible reflectance spectroscopy to electron transfer rate measurements for adsorbed species on electrode surfaces. Analytical Chemistry, 1995. 67(19): p. 3564-3570.

36. Katz, E. and I. Willner, Probing Biomolecular Interactions at Conductive and Semiconductive Surfaces by Impedance Spectroscopy: Routes to Impedimetric 
Immunosensors, DNA-Sensors, and Enzyme Biosensors. Electroanalysis, 2003. 15(11): p. 913-947.

37. Niu, J. and S. Dong, Transmission Spectroelectrochemistry, in Reviews in Analytical Chemistry. 1996. p. 1.

38. Araci, Z.O., et al., Potential-Modulated Attenuated Total Reflectance Characterization of Charge Injection Processes in Monolayer-Tethered CdSe Nanocrystals. The Journal of Physical Chemistry Letters, 2010. 1(12): p. 1900-1905.

39. Doherty, W.J., et al., Potential-Modulated, Attenuated Total Reflectance Spectroscopy of Poly(3,4-ethylenedioxythiophene) and Poly(3,4-ethylenedioxythiophene Methanol) Copolymer Films on Indium-Tin Oxide. The Journal of Physical Chemistry B, 2006. 110(10): p. 4900-4907.

40. Brockman, J.M., B.P. Nelson, and R.M. Corn, Surface Plasmon Resonance Imaging Measurements of Ultrathin Organic Films. Annual Review of Physical Chemistry, 2000. 51(1): p. 41-63.

41. Del Rosso, T., et al., Accurate and simultaneous measurement of thickness and refractive index of thermally evaporated thin organic films by surface plasmon resonance spectroscopy. Optics Express, 2014. 22(16): p. 18914-18923.

42. Granqvist, N., et al., Characterizing Ultrathin and Thick Organic Layers by Surface Plasmon Resonance Three-Wavelength and Waveguide Mode Analysis. Langmuir, 2013. 29(27): p. 8561-8571.

43. Luna-Moreno, D., et al., Determination of quality and adulteration of tequila through the use of surface plasmon resonance. Applied Optics, 2012. 51(21): p. 5161-5167.

44. El-Denglawey, A., Characterization of As-Se-TI films near infrared region. Journal of Non-Crystalline Solids, 2011. 357(7): p. 1757-1763.

45. Allsop, T., et al., Physical characteristics of localized surface plasmons resulting from nano-scale structured multi-layer thin films deposited on D-shaped optical fiber. Optics Express, 2013. 21(16): p. 18765-18776.

46. Lakowicz, J.R., et al., Plasmon-controlled fluorescence: a new paradigm in fluorescence spectroscopy. Analyst, 2008. 133(10): p. 1308-1346.

47. Kretschmann, E. and H. Raether, Notizen: Radiative Decay of Non Radiative Surface Plasmons Excited by Light, in Zeitschrift für Naturforschung A. 1968. p. 2135.

48. Otto, A., Excitation of nonradiative surface plasma waves in silver by the method of frustrated total reflection. Zeitschrift für Physik A Hadrons and nuclei, 1968. 216(4): p. 398-410.

49. Kretschmann, E., Decay of non radiative surface plasmons into light on rough silver films. Comparison of experimental and theoretical results. Optics Communications, 1972. 6(2): p. 185-187.

50. Su, W., G. Zheng, and X. Li, Design of a highly sensitive surface plasmon resonance sensor using aluminum-based diffraction grating. Optics Communications, 2012. 285(21): p. 4603-4607.

51. Maharana, P.K., T. Srivastava, and R. Jha, Ultrasensitive Plasmonic Imaging Sensor Based on Graphene and Silicon. IEEE Photonics Technology Letters, 2013. 25(2): p. 122-125.

52. Sharma, A.K., R. Jha, and B.D. Gupta, Fiber-Optic Sensors Based on Surface Plasmon Resonance: A Comprehensive Review. IEEE Sensors Journal, 2007. 7(8): p. 1118-1129.

53. Sreekanth, K.V., et al., Enhancing the Angular Sensitivity of Plasmonic Sensors Using Hyperbolic Metamaterials. Advanced optical materials, 2016. 4(11): p. 1767-1772.

54. Nylander, C., B. Liedberg, and T. Lind, Gas detection by means of surface plasmon resonance. Sensors and Actuators, 1982. 3: p. 79-88. 
55. Englebienne, P., A.V. Hoonacker, and M. Verhas, Surface plasmon resonance: principles, methods and applications in biomedical sciences. Spectroscopy, 2003. 17(2,3): p. 255273.

56. Navratilova, I. and A.L. Hopkins, Emerging role of surface plasmon resonance in fragment-based drug discovery. Future Med Chem, 2011. 3(14): p. 1809-20.

57. Bergwerff, A.A. and F. van Knapen, Surface plasmon resonance biosensors for detection of pathogenic microorganisms: strategies to secure food and environmental safety. J AOAC Int, 2006. 89(3): p. 826-31.

58. Thillaivinayagalingam, P., et al., Biopharmaceutical production: Applications of surface plasmon resonance biosensors. J Chromatogr B Analyt Technol Biomed Life Sci, 2010. 878(2): p. 149-53.

59. Haughey, S.A., et al., Comparison of biosensor platforms for surface plasmon resonance based detection of paralytic shellfish toxins. Talanta, 2011. 85(1): p. 519-526.

60. Velusamy, V., et al., An overview of foodborne pathogen detection: In the perspective of biosensors. Biotechnology Advances, 2010. 28(2): p. 232-254.

61. Arora, P., et al., Biosensors as innovative tools for the detection of food borne pathogens. Biosensors and Bioelectronics, 2011. 28(1): p. 1-12.

62. Kawazumi, H., et al., Compact surface plasmon resonance (SPR) immunosensor using multichannel for simultaneous detection of small molecule compounds. Sensors and Actuators B: Chemical, 2005. 108(1): p. 791-796.

63. Piliarik, M., H. Vaisocherova, and J. Homola, A new surface plasmon resonance sensor for high-throughput screening applications. Biosens Bioelectron, 2005. 20(10): p. 210410.

64. Jordan, C.E., et al., Surface Plasmon Resonance Imaging Measurements of DNA Hybridization Adsorption and Streptavidin/DNA Multilayer Formation at Chemically Modified Gold Surfaces. Analytical Chemistry, 1997. 69(24): p. 4939-4947.

65. Jordan, C.E. and R.M. Corn, Surface Plasmon Resonance Imaging Measurements of Electrostatic Biopolymer Adsorption onto Chemically Modified Gold Surfaces. Anal Chem, 1997. 69(7): p. 1449-56.

66. Shumaker-Parry, J.S. and C.T. Campbell, Quantitative methods for spatially resolved adsorption/desorption measurements in real time by surface plasmon resonance microscopy. Anal Chem, 2004. 76(4): p. 907-17.

67. Campbell, C.T. and G. Kim, SPR microscopy and its applications to high-throughput analyses of biomolecular binding events and their kinetics. Biomaterials, 2007. 28(15): p. 2380-92.

68. Piliarik, M. and J. Homola, Surface plasmon resonance (SPR) sensors: approaching their limits? Optics Express, 2009. 17(19): p. 16505-16517.

69. Homola, J., Surface Plasmon Resonance Sensors for Detection of Chemical and Biological Species. Chemical Reviews, 2008. 108(2): p. 462-493.

70. Notcovich, A.G., V. Zhuk, and S.G. Lipson, Surface plasmon resonance phase imaging. Applied Physics Letters, 2000. 76(13): p. 1665-1667.

71. $\mathrm{Wu}, \mathrm{C} .-\mathrm{M}$., et al., High-sensitivity sensor based on surface plasmon resonance and heterodyne interferometry. Sensors and Actuators B: Chemical, 2003. 92(1): p. 133-136.

72. Shao, Y., et al., Wavelength-multiplexing phase-sensitive surface plasmon imaging sensor. Optics Letters, 2013. 38(9): p. 1370-1372.

73. Byard, C.L., X. Han, and S.B. Mendes, Angle-Multiplexed Waveguide Resonance of High Sensitivity and Its Application to Nanosecond Dynamics of Molecular Assemblies. Analytical Chemistry, 2012. 84(22): p. 9762-9767. 
74. Boussaad, S., J. Pean, and N.J. Tao, High-Resolution Multiwavelength Surface Plasmon Resonance Spectroscopy for Probing Conformational and Electronic Changes in Redox Proteins. Analytical Chemistry, 2000. 72(1): p. 222-226.

75. Tao, N.J., et al., High resolution surface plasmon resonance spectroscopy. Review of Scientific Instruments, 1999. 70(12): p. 4656-4660.

76. Taylor, A.D., et al., Quantitative detection of tetrodotoxin (TTX) by a surface plasmon resonance (SPR) sensor. Sensors and Actuators B: Chemical, 2008. 130(1): p. 120-128.

77. Puiu, M. and C. Bala, SPR and SPR Imaging: Recent Trends in Developing Nanodevices for Detection and Real-Time Monitoring of Biomolecular Events. Sensors, 2016. 16(6).

78. Liang, $\mathrm{H}$., et al., Surface plasmon resonance instrument as a refractometer for liquids and ultrathin films. Sensors and Actuators B: Chemical, 2010. 149(1): p. 212-220.

79. Nguyen, H.H., et al., Surface plasmon resonance: a versatile technique for biosensor applications. Sensors (Basel), 2015. 15(5): p. 10481-510.

80. Zeng, S., et al., A Review on Functionalized Gold Nanoparticles for Biosensing Applications. Plasmonics, 2011. 6(3): p. 491.

81. Wang, S., et al., Electrochemical surface plasmon resonance: basic formalism and experimental validation. Analytical chemistry, 2010. 82(3): p. 935-41.

82. Wang, S., et al., Electrochemical Surface Plasmon Resonance: Basic Formalism and Experimental Validation. Analytical Chemistry, 2010. 82(3): p. 935-941.

83. Lu, J., et al., Plasmonic-Based Electrochemical Impedance Spectroscopy: Application to Molecular Binding. Analytical Chemistry, 2012. 84(1): p. 327-333.

84. Lu, J. and J. Li, Charge Transfer Kinetics from Surface Plasmon Resonance Voltammetry. Analytical Chemistry, 2014. 86(8): p. 3882-3886.

85. Kötz, R., D.M. Kolb, and J.K. Sass, Electron density effects in surface plasmon excitation on silver and gold electrodes. Surface Science, 1977. 69(1): p. 359-364.

86. Gordon, J.G. and S. Ernst, Surface plasmons as a probe of the electrochemical interface. Surface Science, 1980. 101(1): p. 499-506.

87. Wang, S., et al., High-sensitivity stark spectroscopy obtained by surface plasmon resonance measurement. Anal Chem, 2000. 72(17): p. 4003-8.

88. Foley, K.J., X. Shan, and N.J. Tao, Surface Impedance Imaging Technique. Analytical Chemistry, 2008. 80(13): p. 5146-5151.

89. Liang, W., et al., Measurement of Small Molecule Binding Kinetics on a Protein Microarray by Plasmonic-Based Electrochemical Impedance Imaging. Analytical Chemistry, 2014. 86(19): p. 9860-9865.

90. MacGriff, C., et al., Charge-Based Detection of Small Molecules by Plasmonic-Based Electrochemical Impedance Microscopy. Analytical Chemistry, 2013. 85(14): p. 66826687.

91. Shan, X., et al., Detection of Charges and Molecules with Self-Assembled NanoOscillators. Nano Letters, 2014. 14(7): p. 4151-4157.

92. Dallaire, A.-M., et al., Electrochemical plasmonic sensing system for highly selective multiplexed detection of biomolecules based on redox nanoswitches. Biosensors and Bioelectronics, 2015. 71: p. 75-81.

93. Yakubovsky, D.I., et al., Optical constants and structural properties of thin gold films. Opt Express, 2017. 25(21): p. 25574-25587.

94. Johnson, P.B. and R.W. Christy, Optical Constants of the Noble Metals. Physical Review B, 1972. 6(12): p. 4370-4379.

95. Palik, E.D., Handbook of optical constants of solids. 1985, Orlando: Academic Press. 
96. Shushama, K.N., et al., Sensitivity enhancement of graphene coated surface plasmon resonance biosensor. Optical and Quantum Electronics, 2017. 49(11): p. 381.

97. Zhai, P., et al., Electrochemical Surface Plasmon Resonance Spectroscopy at Bilayered Silver/Gold Films. The Journal of Physical Chemistry C, 2007. 111(2): p. 981-986.

98. Michota-Kaminska, A., B. Wrzosek, and J. Bukowska, Resonance Raman Evidence of Immobilization of Laccase on Self-Assembled Monolayers of Thiols on Ag and $\mathrm{Au}$ Surfaces. Applied Spectroscopy, 2006. 60(7): p. 752-757.

99. Mendes, R.K., et al., Characterization of self-assembled thiols monolayers on gold surface by electrochemical impedance spectroscopy. Journal of the Brazilian Chemical Society, 2004. 15: p. 849-855.

100. Wink, T., et al., Self-assembled monolayers for biosensors. The Analyst, 1997. 122(4): p. 43R-50R.

101. Grunwald, P., Immobilized Biocatalysts. 2018: MDPI AG.

102. Arya, S.K., et al., Recent advances in self-assembled monolayers based biomolecular electronic devices. Biosens Bioelectron, 2009. 24(9): p. 2810-7.

103. Altintas, Z., et al., Development of surface chemistry for surface plasmon resonance based sensors for the detection of proteins and DNA molecules. Anal Chim Acta, 2012. 712: p. 138-44.

104. Liu, S., D.n. Leech, and H. Ju, Application of Colloidal Gold in Protein Immobilization, Electron Transfer, and Biosensing. Analytical Letters, 2003. 36(1): p. 1-19.

105. Wadu-Mesthrige, K., N.A. Amro, and G.Y. Liu, Immobilization of proteins on selfassembled monolayers. Scanning, 2000. 22(6): p. 380-8.

106. Crilly, S. and E. Magner, Reversible increase in the redox potential of cytochrome $c$ in methanol. Chemical Communications, 2009(5): p. 535-537.

107. López-Bernabeu, S., et al., Direct Electron Transfer to Cytochrome c Induced by a Conducting Polymer. The Journal of Physical Chemistry C, 2017. 121(29): p. 1587015879.

108. Fedurco, M., Redox reactions of heme-containing metalloproteins: dynamic effects of self-assembled monolayers on thermodynamics and kinetics of cytochrome c electrontransfer reactions. Coordination Chemistry Reviews, 2000. 209(1): p. 263-331.

109. Bard, A.J. and L.R. Faulkner, Electrochemical methods : fundamentals and applications. 2nd ed. ed. 2001, New York: Wiley.

110. Li, N., et al., The Direct Electron Transfer of Myoglobin Based on the Electron Tunneling in Proteins. The Journal of Physical Chemistry B, 2006. 110(23): p. 11561-11565.

111. El Kasmi, A., et al., Controlling Interfacial Electron-Transfer Kinetics of Cytochrome $c$ with Mixed Self-Assembled Monolayers. Journal of the American Chemical Society, 1998. 120(1): p. 225-226.

112. Chen, R., et al., Electron transfer-based single molecule fluorescence as a probe for nanoenvironment dynamics. Sensors (Basel), 2014. 14(2): p. 2449-67.

113. Yue, H. and D.H. Waldeck, Understanding interfacial electron transfer to monolayer protein assemblies. Current Opinion in Solid State and Materials Science, 2005. 9(1): p. 28-36.

114. Pan, S., et al., Design and control of electron transport properties of single molecules. Proceedings of the National Academy of Sciences, 2009. 106(36): p. 15259-15263.

115. Willner, I. and B. Willner, Biomaterials integrated with electronic elements: en route to bioelectronics. Trends in Biotechnology, 2001. 19(6): p. 222-230. 
116. Kakehi, N., et al., A novel wireless glucose sensor employing direct electron transfer principle based enzyme fuel cell. Biosensors and Bioelectronics, 2007. 22(9): p. 22502255.

117. Wei, J., et al., Direct Wiring of Cytochrome c's Heme Unit to an Electrode: Electrochemical Studies. Journal of the American Chemical Society, 2002. 124(32): p. 9591-9599.

118. Willner, I., et al., Assembly of functionalized monolayers of redox proteins on electrode surfaces: novel bioelectronic and optobioelectronic systems. Biosensors and Bioelectronics, 1997. 12(4): p. 337-356.

119. Gaspar, S., et al., Hydrogen Peroxide Biosensors Based on Direct Electron Transfer from Plant Peroxidases Immobilized on Self-Assembled Thiol-Monolayer Modified Gold Electrodes. Electroanalysis, 2001. 13(4): p. 284-288.

120. Liu, H., et al., Control of the Electron Transfer Rate between Cytochrome c and Gold Electrodes by the Manipulation of the Electrode's Hydrogen Bonding Character. Langmuir, 2003. 19(6): p. 2378-2387.

121. Yue, H., et al., Multiple Sites for Electron Tunneling between Cytochrome $c$ and Mixed Self-Assembled Monolayers. The Journal of Physical Chemistry C, 2008. 112(7): p. 25142521.

122. de Groot, M.T., et al., Electron transfer and ligand binding to cytochrome c' immobilized on self-assembled monolayers. Langmuir, 2007. 23(2): p. 729-36.

123. Chen, X., et al., Redox Properties of Cytochrome c Adsorbed on Self-Assembled Monolayers: A Probe for Protein Conformation and Orientation. Langmuir, 2002. 18(18): p. 7009-7015.

124. Kong, F. and Y.F. Hu, Biomolecule immobilization techniques for bioactive paper fabrication. Anal Bioanal Chem, 2012. 403(1): p. 7-13.

125. Ulman, A., Formation and Structure of Self-Assembled Monolayers. Chemical Reviews, 1996. 96(4): p. 1533-1554.

126. Zhang, P., et al., Surface plasmon resonance for water pollutant detection and water process analysis. TrAC Trends in Analytical Chemistry, 2016. 85: p. 153-165.

127. Yagati, A.K., et al., Amperometric sensor for hydrogen peroxide based on direct electron transfer of spinach ferredoxin on Au electrode. Bioelectrochemistry, 2011. 80(2): p. 169174.

128. Carmona-Martinez, A.A., et al., Cyclic voltammetric analysis of the electron transfer of Shewanella oneidensis MR-1 and nanofilament and cytochrome knock-out mutants. Bioelectrochemistry (Amsterdam, Netherlands), 2011. 81(2): p. 74-80.

129. Campos, R. and E.E. Ferapontova, Electrochemistry of weakly adsorbed species: Voltammetric analysis of electron transfer between gold electrodes and Ru hexaamine electrostatically interacting with DNA duplexes. Electrochimica Acta, 2014. 126: p. 151157.

130. Ito, Y., et al., Third generation impedimetric sensor employing direct electron transfer type glucose dehydrogenase. Biosensors \& bioelectronics, 2019. 129: p. 189-197.

131. Asav, E. and M.K. Sezgintürk, A novel impedimetric disposable immunosensor for rapid detection of a potential cancer biomarker. International journal of biological macromolecules, 2014. 66: p. 273-80.

132. Eckermann, A.L., et al., Electrochemistry of redox-active self-assembled monolayers. Coordination chemistry reviews, 2010. 254(15-16): p. 1769-1802.

133. Han, X. and S.B. Mendes, Electron-Transfer Rate in Potential-Modulated Redox Reactions with Electro-Active Optical Waveguides. Anal Sci, 2017. 33(4): p. 435-441. 
134. Han, X. and S.B. Mendes, Optical Impedance Spectroscopy with Single-Mode ElectroActive-Integrated Optical Waveguides. Analytical Chemistry, 2014. 86(3): p. 1468-1477.

135. Fang, Y., et al., Plasmonic Imaging of Electrochemical Reactions of Single Nanoparticles. Accounts of Chemical Research, 2016. 49(11): p. 2614-2624.

136. Samanta, D. and A. Sarkar, Immobilization of bio-macromolecules on self-assembled monolayers: methods and sensor applications. Chemical Society Reviews, 2011. 40(5): p. 2567-2592.

137. Tichoniuk, M., M. Ligaj, and M. Filipiak, Application of DNA Hybridization Biosensor as a Screening Method for the Detection of Genetically Modified Food Components. Sensors (Basel), 2008. 8(4): p. 2118-2135.

138. Ignat, T., et al., Electrochemical characterization of BSA/11-mercaptoundecanoic acid on Au electrode. Materials Science and Engineering: B, 2010. 169(1): p. 55-61.

139. Phan, H.T.M., et al., Investigation of Bovine Serum Albumin (BSA) Attachment onto SelfAssembled Monolayers (SAMs) Using Combinatorial Quartz Crystal Microbalance with Dissipation (QCM-D) and Spectroscopic Ellipsometry (SE). PloS one, 2015. 10(10): p. e0141282-e0141282.

140. Armstrong, F.A., et al., Fast, long-range electron-transfer reactions of a 'blue' copper protein coupled non-covalently to an electrode through a stilbenyl thiolate monolayer. Chemical Communications, 2004(3): p. 316-317.

141. Fujita, K., et al., Mimicking Protein-Protein Electron Transfer: Voltammetry of Pseudomonas aeruginosa Azurin and the Thermus thermophilus CuA Domain at $\omega$ Derivatized Self-Assembled-Monolayer Gold Electrodes. Journal of the American Chemical Society, 2004. 126(43): p. 13954-13961.

142. Clark, R.A. and E.F. Bowden, Voltammetric Peak Broadening for Cytochrome c/Alkanethiolate Monolayer Structures: Dispersion of Formal Potentials. Langmuir, 1997. 13(3): p. 559-565.

143. Collinson, M., E.F. Bowden, and M.J. Tarlov, Voltammetry of covalently immobilized cytochrome $c$ on self-assembled monolayer electrodes. Langmuir, 1992. 8(5): p. 12471250.

144. Freire, R.S., et al., Direct electron transfer: an approach for electrochemical biosensors with higher selectivity and sensitivity. Journal of the Brazilian Chemical Society, 2003. 14: p. 230-243.

145. Liu, Y. and J. Yu, Oriented immobilization of proteins on solid supports for use in biosensors and biochips: a review. Microchimica Acta, 2016. 183(1): p. 1-19.

146. Sargent, A., et al., The electrochemistry of antibody-modified conducting polymer electrodes. Journal of Electroanalytical Chemistry, 1999. 470(2): p. 144-156.

147. Rabe, M., D. Verdes, and S. Seeger, Understanding protein adsorption phenomena at solid surfaces. Advances in Colloid and Interface Science, 2011. 162(1): p. 87-106.

148. Bostick, C.D., et al., Protein bioelectronics: a review of what we do and do not know. Rep Prog Phys, 2018. 81(2): p. 026601.

149. Davis, K.L., et al., Electron-Transfer Kinetics of Covalently Attached Cytochrome c/SAM/Au Electrode Assemblies. The Journal of Physical Chemistry C, 2008. 112(16): p. 6571-6576.

150. Feng, B., et al., Site-oriented immobilization of fusion antigen directed by an affinity ligand, and its validation in an immunoassay. Surface and Interface Analysis, 2011. 43(10): p. 1304-1310. 
151. Organization, W.H. Rolling updates on coronavirus disease (COVID-19). 2020; Available from: https://www.who.int/emergencies/diseases/novel-coronavirus-2019/events-asthey-happen.

152. Prevention, C.f.D.C.a. Chapter 6: Influenza. November 10, 2017; Available from: https://www.cdc.gov/vaccines/pubs/surv-manual/chpt06-influenza.html.

153. Sakai, T., et al., Influenza $A$ virus hemagglutinin and neuraminidase act as novel motile machinery. Scientific Reports, 2017. 7(1): p. 45043.

154. Zhang, J. and J. Zhao, Chapter 5 -Immuno-Biosensor, in Nano-Inspired Biosensors for Protein Assay with Clinical Applications, G. Li, Editor. 2019, Elsevier. p. 115-137.

155. Anandan, V., R. Gangadharan, and G. Zhang, Role of SAM Chain Length in Enhancing the Sensitivity of Nanopillar Modified Electrodes for Glucose Detection. Sensors (Basel, Switzerland), 2009. 9(3): p. 1295-1305.

156. Beeram, S.R. and F.P. Zamborini, Effect of Protein Binding Coverage, Location, and Distance on the Localized Surface Plasmon Resonance Response of Purified Au Nanoplates Grown Directly on Surfaces. The Journal of Physical Chemistry C, 2011. 115(15): p. 7364-7371.

157. Salant, A., E. Amitay-Sadovsky, and U. Banin, Directed Self-Assembly of Gold-Tipped CdSe Nanorods. Journal of the American Chemical Society, 2006. 128(31): p. 10006-10007.

158. Li, X., et al., Light-Induced Selective Deposition of Metals on Gold-Tipped CdSe-Seeded CdS Nanorods. Journal of the American Chemical Society, 2011. 133(4): p. 672-675.

159. Chang, Y.F., et al., Use of liposomal amplifiers in total internal reflection fluorescence fiber-optic biosensors for protein detection. Biosensors \& bioelectronics, 2016. 77: p. 1201-7.

160. Engström, H.A., P.O. Andersson, and S. Ohlson, A label-free continuous total-internalreflection-fluorescence-based immunosensor. Analytical Biochemistry, 2006. 357(2): p. 159-166.

161. Krieg, A., et al., Real-Time Detection of Polymerase Activity Using Supercritical Angle Fluorescence. Journal of Fluorescence, 2004. 14(1): p. 75-78.

162. Hung, T.Q., et al., A novel lab-on-chip platform with integrated solid phase PCR and Supercritical Angle Fluorescence (SAF) microlens array for highly sensitive and multiplexed pathogen detection. Biosensors and Bioelectronics, 2017. 90: p. 217-223.

163. Ruckstuhl, T., C.M. Winterflood, and S. Seeger, Supercritical angle fluorescence immunoassay platform. Analytical chemistry, 2011. 83(6): p. 2345-50.

164. Matveeva, E., et al., Immunoassays based on directional surface plasmon-coupled emission. Journal of Immunological Methods, 2004. 286(1): p. 133-140.

165. Matveeva, E.G., et al., Directional surface plasmon-coupled emission: Application for an immunoassay in whole blood. Analytical Biochemistry, 2005. 344(2): p. 161-167.

166. Bauch, M., et al., Plasmon-Enhanced Fluorescence Biosensors: a Review. Plasmonics, 2014. 9(4): p. 781-799. 


\section{APPENDICES}

Appendix A: EC-SPR Characterization

1. Mathematica Program for Calculating the thickness and the optical constants of a thin

film Clear $[\lambda, \varphi, \phi, \alpha, \mathrm{x}, \mathrm{y}, \mathrm{z}]$

"Wavelength (nm)";

$\lambda=660$;

"Base angle of prism in degrees";

$\varphi=60$;

"Refractive outside prism, typically air";

$\mathrm{n}_{\text {air }}=1.000$;

"Medium before layers, BK-7";

$\mathrm{n}_{0}=\left(1+1.03961212 /\left(1-0.00600069867 /(\lambda / 1000)^{\wedge} 2\right)+0.231792344 /(1-\right.$

$\left.\left.0.0200179144 /(\lambda / 1000)^{\wedge} 2\right)+1.01046945 /\left(1-103.560653 /(\lambda / 1000)^{\wedge} 2\right)\right)^{\wedge} .5 ;$

"Layer 1";

$\mathrm{n}_{1}=\mathrm{x}-\mathrm{y} \mathrm{I}$;

$\mathrm{t}_{1}=\mathrm{z}$;

"Layer 2";

$\mathrm{n}_{2}=\mathrm{x}-\mathrm{y} \mathrm{I}$;

$\mathrm{t}_{2}=0$;

"Layer 3";

$\mathrm{n}_{3}=\mathrm{x}-\mathrm{y} \mathrm{I}$;

$\mathrm{t}_{3}=0$;

"Layer 4";

$\mathrm{n}_{4}=\mathrm{x}-\mathrm{y} \mathrm{I}$;

$\mathrm{t}_{4}=0$;

"Layer 5",

$\mathrm{n}_{5}=\mathrm{x}-\mathrm{y} \mathrm{I}$; 
$\mathrm{t}_{5}=0$;

"Medium after layers, e.g. buffer, air";

$\mathrm{n}_{\mathrm{m}}=1.329898027$;

"Read experimental data";

ang=Import["C:/.../Theta","csv"];

ref=Import["C:/.../R","csv"];

$\theta=\operatorname{ang}[[\mathrm{All}, 1]] ;$

$\mathrm{R}=\operatorname{ref}[[\mathrm{All}, 1]]$;

"Start calculations";

$$
\begin{aligned}
& N_{e}=n_{\mathrm{air}} \operatorname{Sin}\left[\frac{\pi}{180} \theta\right] \operatorname{Cos}\left[\frac{\pi}{180} \varphi\right]+\sqrt{\left(n_{0}\right)^{2}-\left(n_{\mathrm{air}} \operatorname{Sin}\left[\frac{\pi}{180} \theta\right]\right)^{2}} \operatorname{Sin}\left[\frac{\pi}{180} \varphi\right] \\
& \chi_{0}=\operatorname{Abs}\left[\operatorname{Re}\left[\sqrt{n_{0}^{2}-N_{e}^{2}}\right]\right]-\operatorname{IAbs}\left[\operatorname{Im}\left[\sqrt{n_{0}^{2}-N_{e}^{2}}\right]\right] \\
& \mathrm{p} \eta_{0}=n_{0}{ }^{2} / \chi_{0} \text {; } \\
& \chi_{1}=\operatorname{Abs}\left[\operatorname{Re}\left[\sqrt{n_{1}^{2}-N_{e}^{2}}\right]\right]-\operatorname{IAbs}\left[\operatorname{Im}\left[\sqrt{n_{1}^{2}-N_{e}^{2}}\right]\right] \\
& \mathrm{p}_{1}=n_{1}{ }^{2} / \chi_{1} ; \\
& \delta_{1}=\frac{2 \pi}{\lambda} t_{1} \chi_{1} ; \\
& \chi_{2}=\operatorname{Abs}\left[\operatorname{Re}\left[\sqrt{n_{2}{ }^{2}-N_{e}{ }^{2}}\right]\right]-\operatorname{IAbs}\left[\operatorname{Im}\left[\sqrt{n_{2}{ }^{2}-N_{e}{ }^{2}}\right]\right] \\
& \mathrm{p} \eta_{2}=n_{2}{ }^{2} / \chi_{2} \text {; } \\
& \delta_{2}=\frac{2 \pi}{\lambda} t_{2} \chi_{2} ; \\
& \chi_{3}=\operatorname{Abs}\left[\operatorname{Re}\left[\sqrt{n_{3}{ }^{2}-N_{e}{ }^{2}}\right]\right]-\operatorname{IAbs}\left[\operatorname{Im}\left[\sqrt{n_{3}^{2}-N_{e}{ }^{2}}\right]\right] \\
& \mathrm{p} \eta_{3}=n_{3}{ }^{2} / \chi_{3} \text {; } \\
& \delta_{3}=\frac{2 \pi}{\lambda} t_{3} \chi_{3} ;
\end{aligned}
$$




$$
\begin{aligned}
& \chi_{4}=\operatorname{Abs}\left[\operatorname{Re}\left[\sqrt{n_{4}{ }^{2}-N_{e}^{2}}\right]\right]-\operatorname{IAbs}\left[\operatorname{Im}\left[\sqrt{n_{4}{ }^{2}-N_{e}}\right]\right] ; \\
& \mathrm{p \eta}_{4}=n_{4}^{2} / \chi_{4} ; \\
& \delta_{4}=\frac{2 \pi}{\lambda} t_{4} \chi_{4} \text {; } \\
& \chi_{5}=\operatorname{Abs}\left[\operatorname{Re}\left[\sqrt{n_{5}{ }^{2}-N_{e}^{2}}\right]\right]-\operatorname{IAbs}\left[\operatorname{Im}\left[\sqrt{n_{5}{ }^{2}-N_{e}}\right]\right] ; \\
& \mathrm{p}_{5}=n_{5}^{2} / \chi_{5} \\
& \delta_{5}=\frac{2 \pi}{\lambda} t_{5} \chi_{5} \text {; } \\
& \chi_{m}=\operatorname{Abs}\left[\operatorname{Re}\left[\sqrt{n_{m}^{2}-N_{e}^{2}}\right]\right]-\operatorname{IAbs}\left[\operatorname{Im}\left[\sqrt{n_{m}^{2}-N_{e}^{2}}\right]\right] ; \\
& \mathrm{p} \eta_{m}=n_{m}{ }^{2} / \chi_{m} ; \\
& \mathrm{M} 1 \mathrm{a}=\operatorname{Cos}\left[\delta_{1}\right] \text {; } \\
& \mathrm{M} 1 \mathrm{~b}=I \operatorname{Sin}\left[\delta_{1}\right] / \mathrm{p \eta}_{1} \text { ； } \\
& \mathrm{M} 1 \mathrm{c}=I \mathrm{p} \eta_{1} \operatorname{Sin}\left[\delta_{1}\right] ; \\
& \operatorname{M} 1 \mathrm{~d}=\operatorname{Cos}\left[\delta_{1}\right] \text {; } \\
& \mathrm{M} 2 \mathrm{a}=\operatorname{Cos}\left[\delta_{2}\right] ; \\
& \mathrm{M} 2 \mathrm{~b}=I \operatorname{Sin}\left[\delta_{2}\right] / \mathrm{p \eta}_{2} \text { ； } \\
& \mathrm{M} 2 \mathrm{c}=I \mathrm{p} \eta_{2} \operatorname{Sin}\left[\delta_{2}\right] ; \\
& \operatorname{M} 2 \mathrm{~d}=\operatorname{Cos}\left[\delta_{2}\right] \text {; } \\
& \mathrm{M} 3 \mathrm{a}=\operatorname{Cos}\left[\delta_{3}\right] \text {; } \\
& \mathrm{M} 3 \mathrm{~b}=I \operatorname{Sin}\left[\delta_{3}\right] / \mathrm{p \eta}_{3} ; \\
& \mathrm{M} 3 \mathrm{c}=I \mathrm{p \eta} \eta_{3} \operatorname{Sin}\left[\delta_{3}\right] ; \\
& \operatorname{M} 3 \mathrm{~d}=\operatorname{Cos}\left[\delta_{3}\right] \text {; } \\
& \mathrm{M} 4 \mathrm{a}=\operatorname{Cos}\left[\delta_{4}\right] ; \\
& \mathrm{M} 4 \mathrm{~b}=I \operatorname{Sin}\left[\delta_{4}\right] / \mathrm{p \eta}_{4} ； \\
& \mathrm{M} 4 \mathrm{c}=I \mathrm{p \eta} \eta_{4} \operatorname{Sin}\left[\delta_{4}\right] ;
\end{aligned}
$$




$$
\begin{aligned}
& \mathrm{M} 4 \mathrm{~d}=\operatorname{Cos}\left[\delta_{4}\right] ; \\
& \mathrm{M} 5 \mathrm{a}=\operatorname{Cos}\left[\delta_{5}\right] ; \\
& \mathrm{M} 5 \mathrm{~b}=I \operatorname{Sin}\left[\delta_{5}\right] / \mathrm{p \eta}_{5} ; \\
& \mathrm{M} 5 \mathrm{c}=I \mathrm{p} \eta_{1} \operatorname{Sin}\left[\delta_{1}\right] ; \\
& \mathrm{M} 5 \mathrm{~d}=\operatorname{Cos}\left[\delta_{5}\right]
\end{aligned}
$$

"Matrix multiplication";

$\mathrm{MLa}=\mathrm{M} 1 \mathrm{a}$;

$\mathrm{MLb}=\mathrm{M} 1 \mathrm{~b} ;$

MLc $=$ M1c;

$\operatorname{MLd}=\mathrm{M} 1 \mathrm{~d}$

$\mathrm{MPa}=\mathrm{MLa} * \mathrm{M} 2 \mathrm{a}+\mathrm{MLb} * \mathrm{M} 2 \mathrm{c} ;$

$\mathrm{MPb}=\operatorname{MLa} * \mathrm{M} 2 \mathrm{~b}+\mathrm{MLb} * \mathrm{M} 2 \mathrm{~d}$;

$\mathrm{MPc}=\mathrm{MLc} * \mathrm{M} 2 \mathrm{a}+\mathrm{MLd} * \mathrm{M} 2 \mathrm{c} ;$

$\mathrm{MPd}=\mathrm{MLc} * \mathrm{M} 2 \mathrm{~b}+\mathrm{MLd} * \mathrm{M} 2 \mathrm{~d} ;$

$\mathrm{MLa}=\mathrm{MPa}$;

$\mathrm{MLb}=\mathrm{MPb}$;

MLac $=$ MPc;

$\operatorname{MLd}=\mathrm{MPd}$;

$\mathrm{MPa}=\mathrm{MLa} * \mathrm{M} 3 \mathrm{a}+\mathrm{MLb} * \mathrm{M} 3 \mathrm{c} ;$

$\mathrm{MPb}=\mathrm{MLa} * \mathrm{M} 3 \mathrm{~b}+\mathrm{MLb} * \mathrm{M} 3 \mathrm{~d} ;$

$\mathrm{MPc}=\mathrm{MLc} * \mathrm{M} 3 \mathrm{a}+\mathrm{MLd} * \mathrm{M} 3 \mathrm{c} ;$

$\operatorname{MPd}=\operatorname{MLc} * \operatorname{M3b}+\operatorname{MLd} * \mathrm{M} 3 \mathrm{~d} ;$

$\mathrm{MLa}=\mathrm{MPa}$;

$\mathrm{MLb}=\mathrm{MPb}$;

MLac $=$ MPc;

$\operatorname{MLd}=\mathrm{MPd}$;

$\mathrm{MPa}=\mathrm{MLa} * \mathrm{M} 4 \mathrm{a}+\mathrm{MLb} * \mathrm{M} 4 \mathrm{c}$;

$\mathrm{MPb}=\operatorname{MLa} *$ M4b + MLb $*$ M4d; 


$$
\begin{aligned}
& \text { MPc }=\text { MLc } * \text { M4a }+ \text { MLd } * \text { M4c; } \\
& \text { MPd }=\text { MLc } * \text { M4b }+ \text { MLd } * \text { M4d; } \\
& \mathrm{MLa}=\mathrm{MPa} \text {; } \\
& \mathrm{MLb}=\mathrm{MPb} \text {; } \\
& \text { MLac }=\text { MPc; } \\
& \text { MLd = MPd; } \\
& \mathrm{MPa}=\mathrm{MLa} * \text { M5a }+ \text { MLb } * \text { M5c; } \\
& \mathrm{MPb}=\text { MLa } * \text { M5b + MLb } * \text { M5d; } \\
& \mathrm{MPc}=\mathrm{MLc} * \mathrm{M} 5 \mathrm{a}+\mathrm{MLd} * \mathrm{M} 5 \mathrm{c} \text {; } \\
& \operatorname{MPd}=\operatorname{MLc} * \text { M5b }+ \text { MLd } * \text { M5d; } \\
& B_{p}=\mathrm{MPa}+\mathrm{p \eta}_{m} \mathrm{MPb} ; \\
& C_{p}=\mathrm{MPc}+\mathrm{p} \eta_{m} \mathrm{MPd} \\
& R_{p}=\operatorname{Re}\left[\frac{\mathrm{p} \eta_{0} B_{p}-C_{p}}{\mathrm{p} \eta_{0} B_{p}+C_{p}} * \text { Conjugate }\left[\frac{\mathrm{p} \eta_{0} B_{p}-C_{p}}{\mathrm{p} \eta_{0} B_{p}+C_{p}}\right]\right] ; \\
& T_{p}=\operatorname{Re}\left[\frac{4 \mathrm{p \eta}_{0} \operatorname{Re}\left[\mathrm{p} \eta_{m}\right]}{\mathrm{p} \eta_{0} B_{p}+C_{p}} * \text { Conjugate }\left[\frac{1}{\mathrm{p} \eta_{0} B_{p}+C_{p}}\right]\right] ; \\
& A_{p}=\operatorname{Re}\left[\frac{4 \mathrm{p} \eta_{0} \operatorname{Re}\left[B_{p} \text { Conjugate }\left[C_{p}\right]-\mathrm{p} \eta_{m}\right]}{\mathrm{p} \eta_{0} B_{p}+C_{p}} * \text { Conjugate }\left[\frac{1}{\mathrm{p} \eta_{0} \quad p+C_{p}}\right]\right] ; \\
& \mathrm{p} \eta_{\mathrm{air}}=n_{\mathrm{air}}^{2} / \sqrt{n_{\mathrm{air}}{ }^{2}-\left(n_{\mathrm{air}} \operatorname{Sin}\left[\frac{\pi}{180} \theta\right]\right)^{2}} \\
& \text { p } \eta_{\text {prism }}=n_{0}^{2} / \sqrt{n_{0}{ }^{2}-\left(n_{\text {air }} \operatorname{Sin}\left[\frac{\pi}{180} \theta\right]\right)^{2}} \text {; }
\end{aligned}
$$

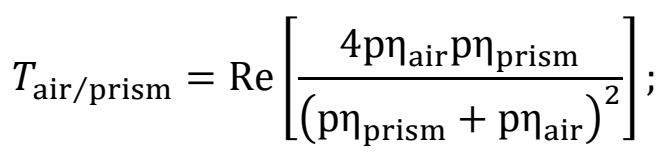

$$
\begin{aligned}
& R_{p}=\operatorname{Re}\left[\frac{\mathrm{p}_{0} B_{p}-C_{p}}{\mathrm{p} \eta_{0} B_{p}+C_{p}} * \text { Conjugate }\left[\frac{\mathrm{p}_{0} B_{p}-C_{p}}{\mathrm{p} \eta_{0} B_{p}+C_{p}}\right]\right]\left(T_{\text {air } / \text { prism }}\right)^{2} ;
\end{aligned}
$$




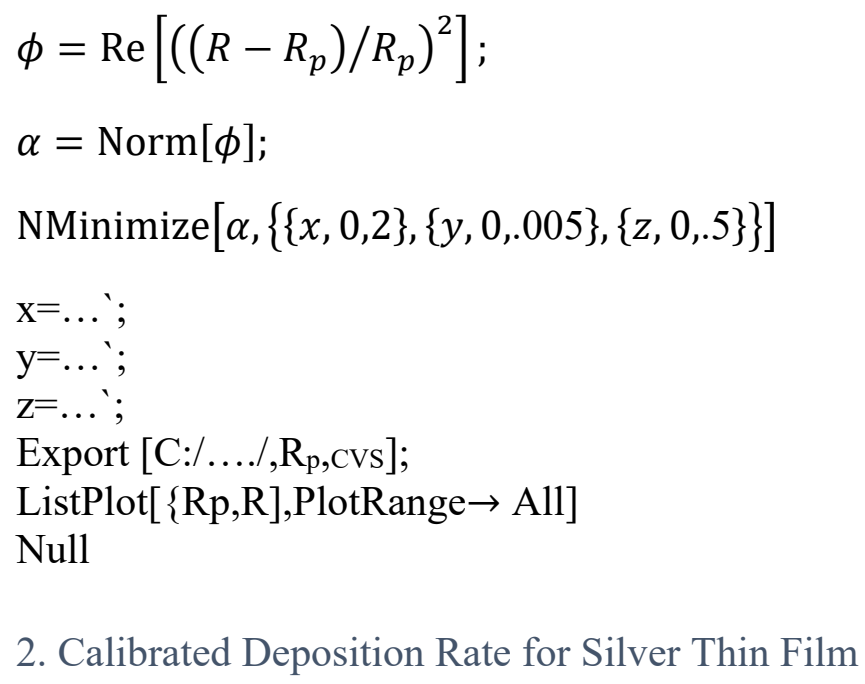

The de deposition rate for silver thin film coating at fixed flow rate of Ar gas and at room temperature was determined. Three different deposition times 30 seconds, 40 seconds, and 50 seconds were used. Figure A-1 shows the measured SPR reflectance curves obtained by the conventional SPR setup, and the calculated SPR reflectance curves based on a three-layer (BK7/silver/air) model at $660 \mathrm{~nm}$ excitation wavelength for three samples. 


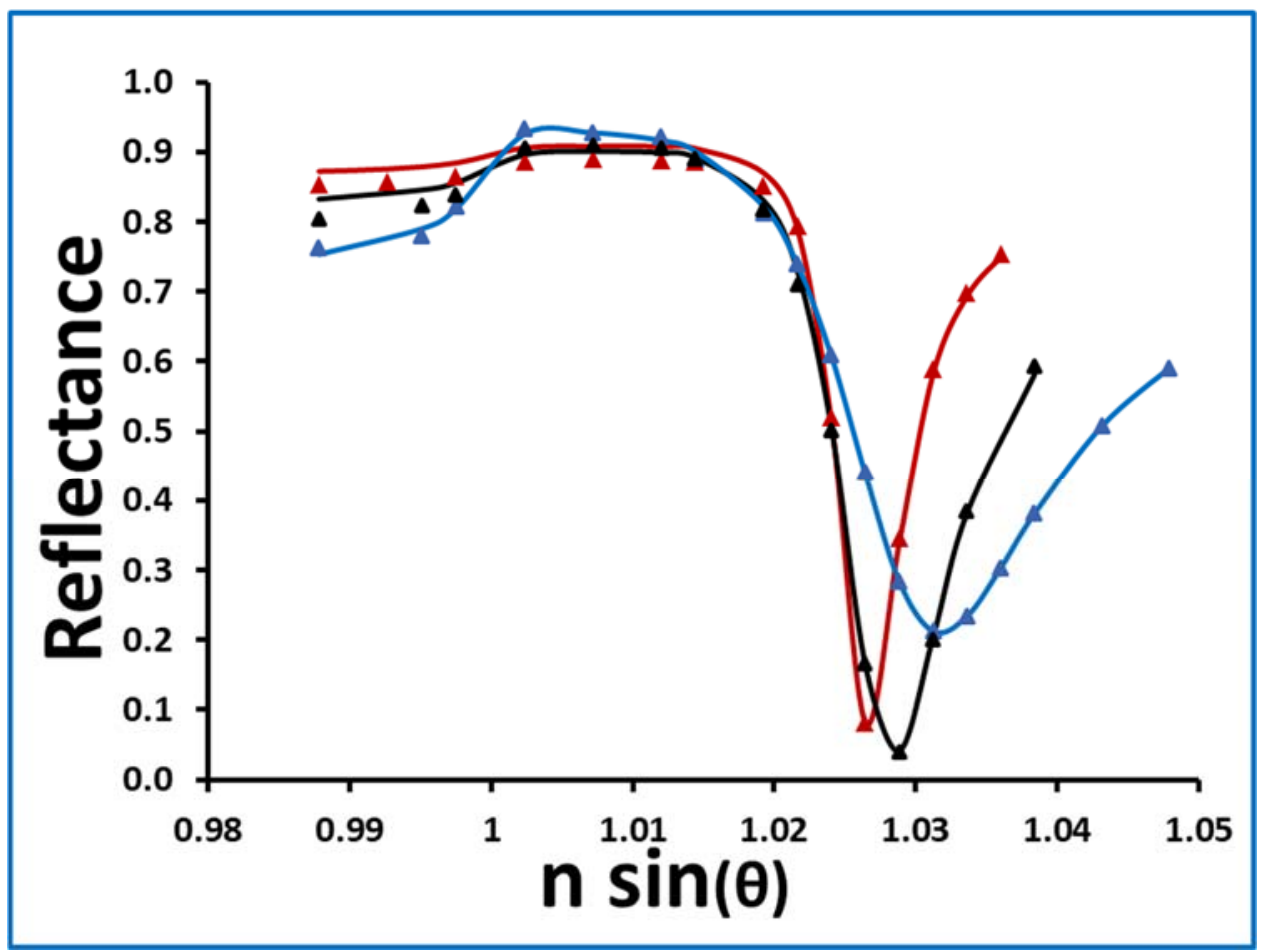

Figure A-1: Measured and calculated SPR reflectance profile to determine the thickness of the silver thin films at three deposition times. The blue, black, and red dots are measured data obtained by the conventional SPR setup at 50 seconds, 40 seconds, and 30 seconds, respectively. The blue, black, and red solid lines are calculated SPR reflectivity curves at 50 seconds, 40 seconds, and 30 seconds, respectively.

The thicknesses of the silver thin film measured at the three deposition times (50 seconds, 40 seconds, and 30 seconds) were $49.8 \mathrm{~nm}, 43.5 \mathrm{~nm}$, and $29.9 \mathrm{~nm}$, respectively. Finally, a plot of the measured silver thickness against the deposition times were used to determine the calibrated rate, Figure A-2. 


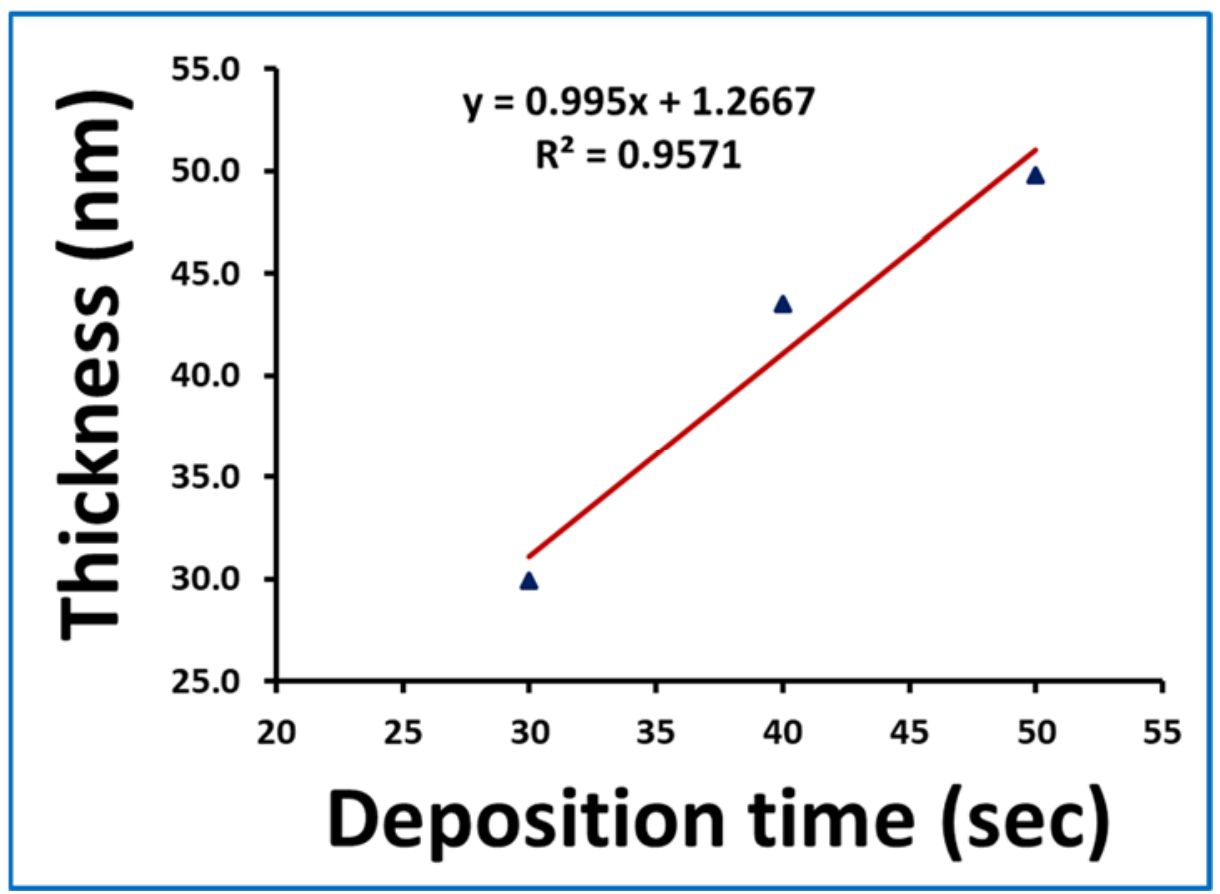

Figure A-2: The measured silver thickness against the deposition time. The deposition rate is represented by the slope of the linear fit.

The calibrated deposition rate to form a thin film of silver using the dc magnetron sputtering technique was measured by a linear fitting to be $1 \mathrm{~nm} / \mathrm{second}$.

3. Mathematica Program for the SPR Reflectivity Calculated of a multilayer model

$$
\operatorname{Clear}\left[n_{0}, n_{m}, N_{e}, M\right]
$$

"Refractive outside prism, typically air";

$\mathrm{n}_{\text {inc }}=1.00$;

"Based angle of prism in degrees";

$\varphi=60$;

"Wavelength (nm)";

$\lambda=785$;

$\mathrm{M}=1000$

$\theta_{\mathrm{i}}=-50$;

$\theta_{\mathrm{f}}=50$;

"Medium before layers, BK-7";

$\mathrm{n}_{0}=\left(1+1.03961212 /\left(1-0.00600069867 /(\lambda / 1000)^{\wedge} 2\right)+0.231792344 /(1-\right.$

$\left.\left.0.0200179144 /(\lambda / 1000)^{\wedge} 2\right)+1.01046945 /\left(1-103.560653 /(\lambda / 1000)^{\wedge} 2\right)\right)^{\wedge} .5$; 


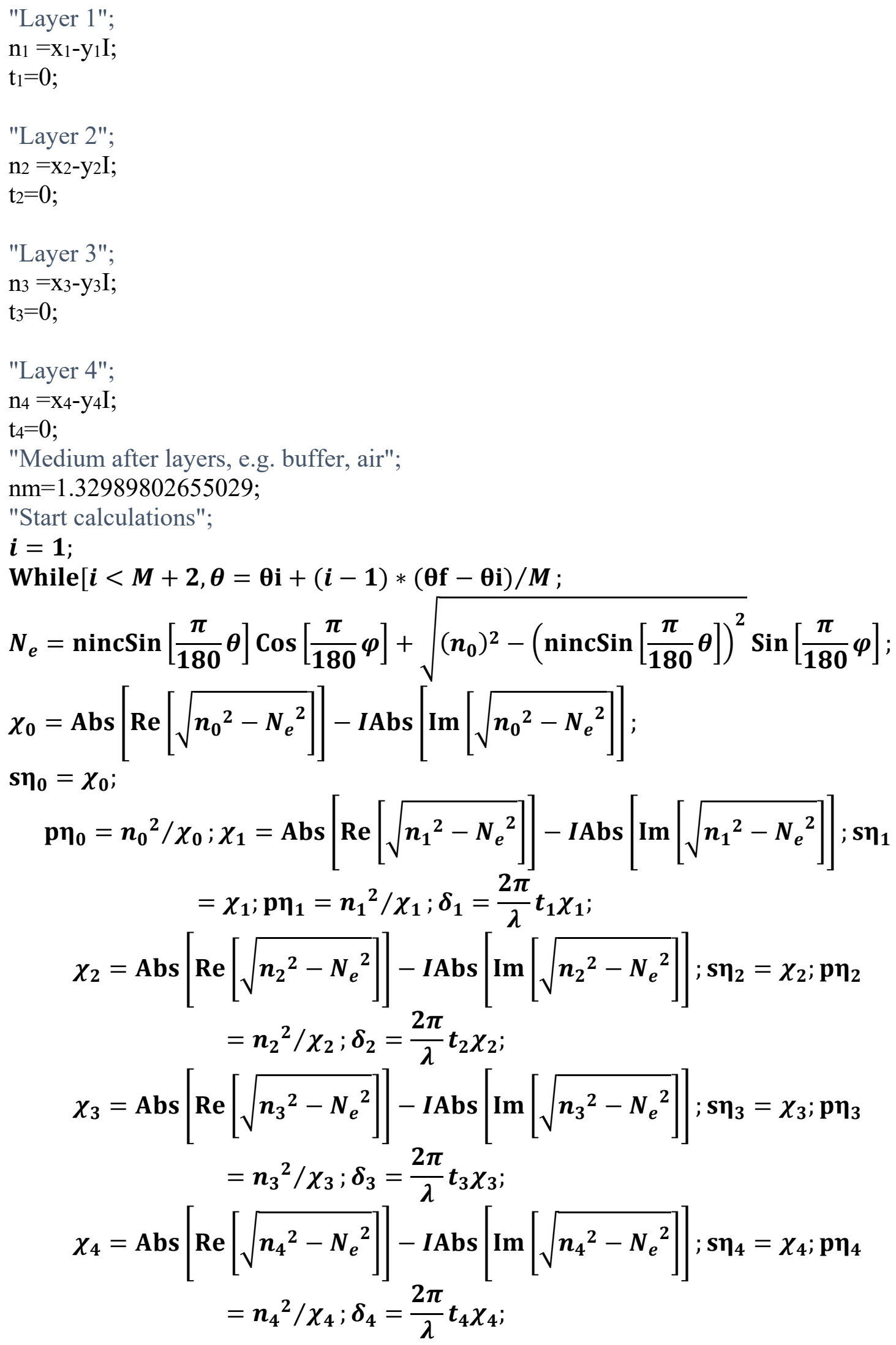




$$
\begin{gathered}
\chi_{m}=\operatorname{Abs}\left[\operatorname{Re}\left[\sqrt{n_{m}^{2}-N_{e}^{2}}\right]\right]-I A b s\left[\operatorname{Im}\left[\sqrt{n_{m}^{2}-N_{e}^{2}}\right] ; s \eta_{m}=\chi_{m} ; \mathbf{p} \eta_{m}\right. \\
=n_{m}{ }^{2} / \chi_{m}
\end{gathered}
$$

$$
M_{p}=\left(\begin{array}{cc}
\operatorname{Cos}\left[\delta_{1}\right] & I \operatorname{Sin}\left[\delta_{1}\right] / p \eta_{1} \\
I p \eta_{1} \operatorname{Sin}\left[\delta_{1}\right] & \operatorname{Cos}\left[\delta_{1}\right]
\end{array}\right) \cdot\left(\begin{array}{cc}
\operatorname{Cos}\left[\delta_{2}\right] & I \operatorname{Sin}\left[\delta_{2}\right] / p \eta_{2} \\
I p \eta_{2} \operatorname{Sin}\left[\delta_{2}\right] & \operatorname{Cos}\left[\delta_{2}\right]
\end{array}\right)
$$

$\left(\begin{array}{cc}\operatorname{Cos}\left[\delta_{3}\right] & I \operatorname{Sin}\left[\delta_{3}\right] / p \eta_{3} \\ I p \eta_{3} \operatorname{Sin}\left[\delta_{3}\right] & \operatorname{Cos}\left[\delta_{3}\right]\end{array}\right) \cdot\left(\begin{array}{cc}\operatorname{Cos}\left[\delta_{4}\right] & I \operatorname{Sin}\left[\delta_{4}\right] / p \eta_{4} \\ I p \eta_{4} \operatorname{Sin}\left[\delta_{4}\right] & \operatorname{Cos}\left[\delta_{4}\right]\end{array}\right)$

$V_{p}=\left(\begin{array}{c}1 \\ \mathbf{p} \eta_{m}\end{array}\right) ; U_{p}=M_{p} . V_{p} ; B_{p}=U_{p}[[1,1]] ; C_{p}=U_{p}[[2,1]]$

$\mathrm{p} \eta_{\mathrm{air}}=n_{i n c}^{2} / \sqrt{n_{i n c}^{2}-\left(n_{i n c} \sin \left[\frac{\pi}{180} \theta\right]\right)^{2}}$

p $\eta_{\text {glass }}=n_{\theta}^{2} / \sqrt{n_{\theta}^{2}-\left(n_{i n c} \sin \left[\frac{\pi}{180} \theta\right]\right)^{2}}$

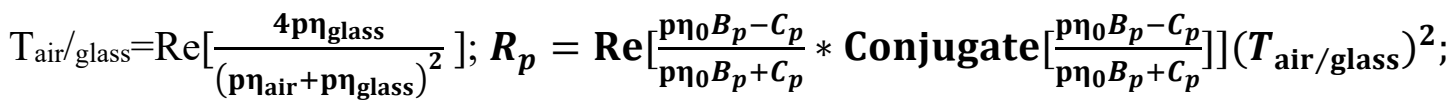

Theta $\left.[i]=\theta ; \operatorname{Neff}[i]=N_{e} ; \operatorname{Rp}[i]=R_{p} ; i++\right]$

$\operatorname{spr} 0=\operatorname{Table}[\{\operatorname{Neff}[i], \operatorname{Theta}[i], \operatorname{Rp}[i]\},\{i, 1, M+1,1\}]$

Export[C:/Users/.../spr0, spr0,csv];

g2 $=\operatorname{ListPlot}[$ Table $[\{\operatorname{Neff}[i], \operatorname{Rp}[i]\},\{i, 0, M, 1\}]$, Joined $\rightarrow$ True, PlotRange $\rightarrow\{\{1,1.52\},\{0,1\}\}$, PlotStyle $\rightarrow$ RGBColor $[1,0,0]$, GridLines $\rightarrow$ Automatic, Frame $\rightarrow$

\section{Show [g2]}

4. Calculated SPR Reflectance Curves for Gold, Silver, and Bilayered (silver/gold) Thin Film
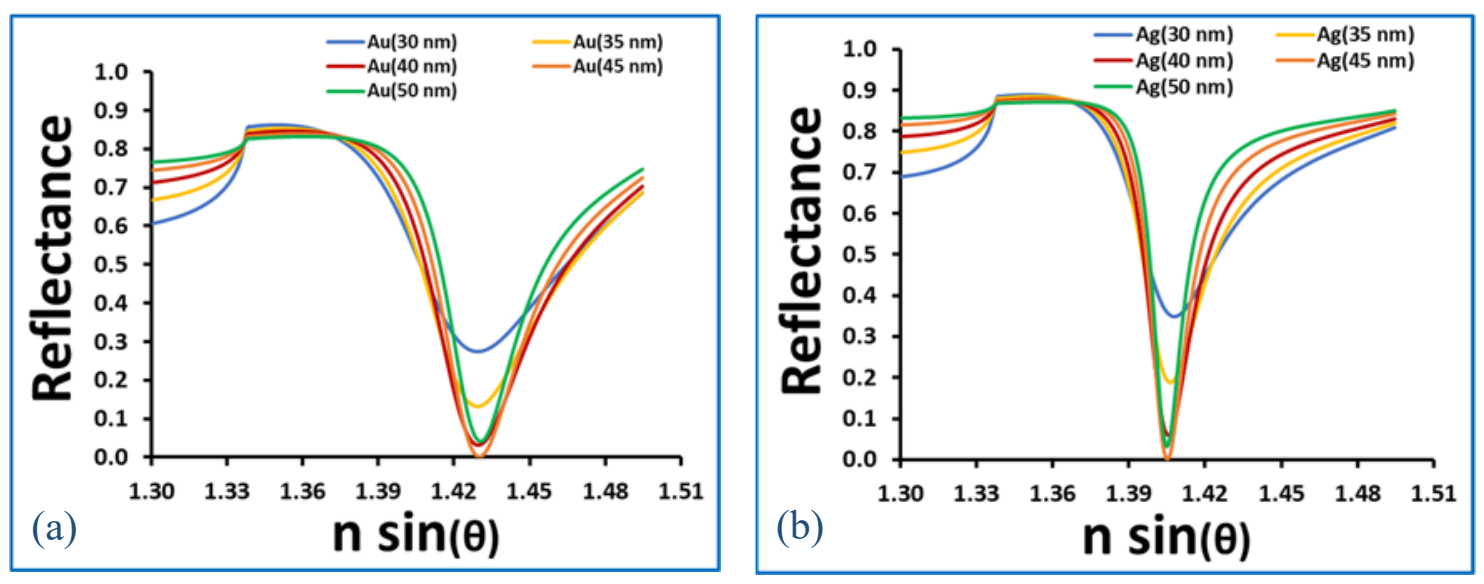


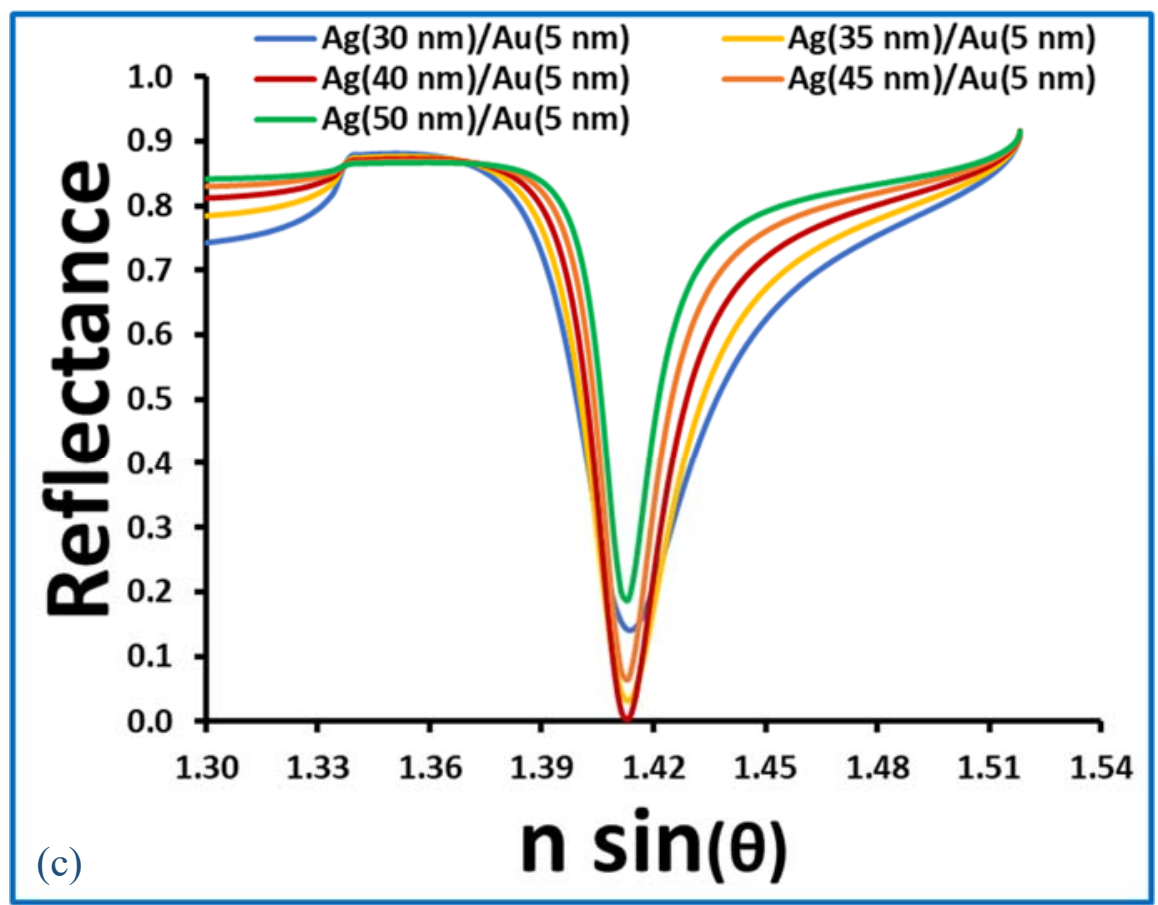

Figure A-3: The calculated SPR reflectance curves for (a) gold thin film, (b) silver thin film with thickness of $30 \mathrm{~nm}, 35 \mathrm{~nm}, 40 \mathrm{~nm}, 45 \mathrm{~nm}$, and $50 \mathrm{~nm}$, and (c) bilayered (silver/gold) thin film with silver thin film thickness of $30 \mathrm{~nm}, 35 \mathrm{~nm}, 40 \mathrm{~nm}, 45 \mathrm{~nm}$, and $50 \mathrm{~nm}$ and a fixed thickness of gold thin film $(5 \mathrm{~nm})$. The following values were used for the calculations: $\mathrm{n}_{\text {prism }}=1.51842, \mathrm{n}_{\text {gold }}=0.38559+2.61439 \mathrm{i}, \mathrm{n}_{\text {silver }}=0.0719+3.2431 \mathrm{i}$, and $\mathrm{n}_{\text {Buffer }}=1.32045$. The excitation wavelength is $637 \mathrm{~nm}$.
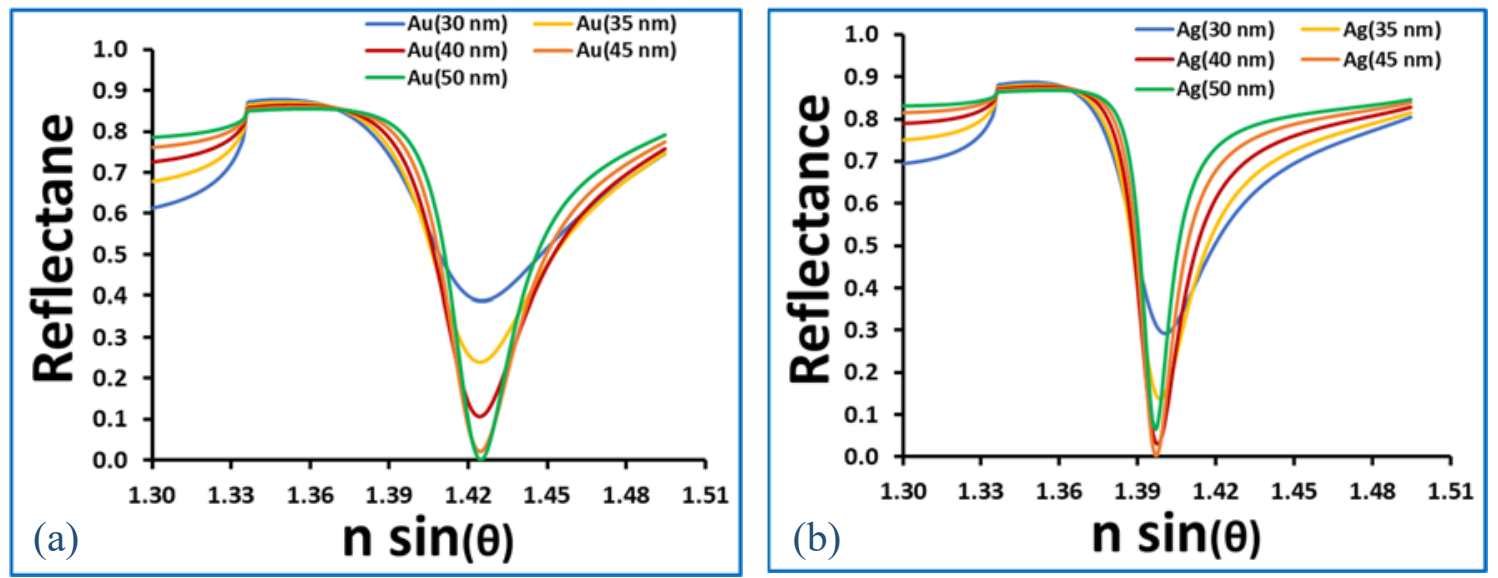


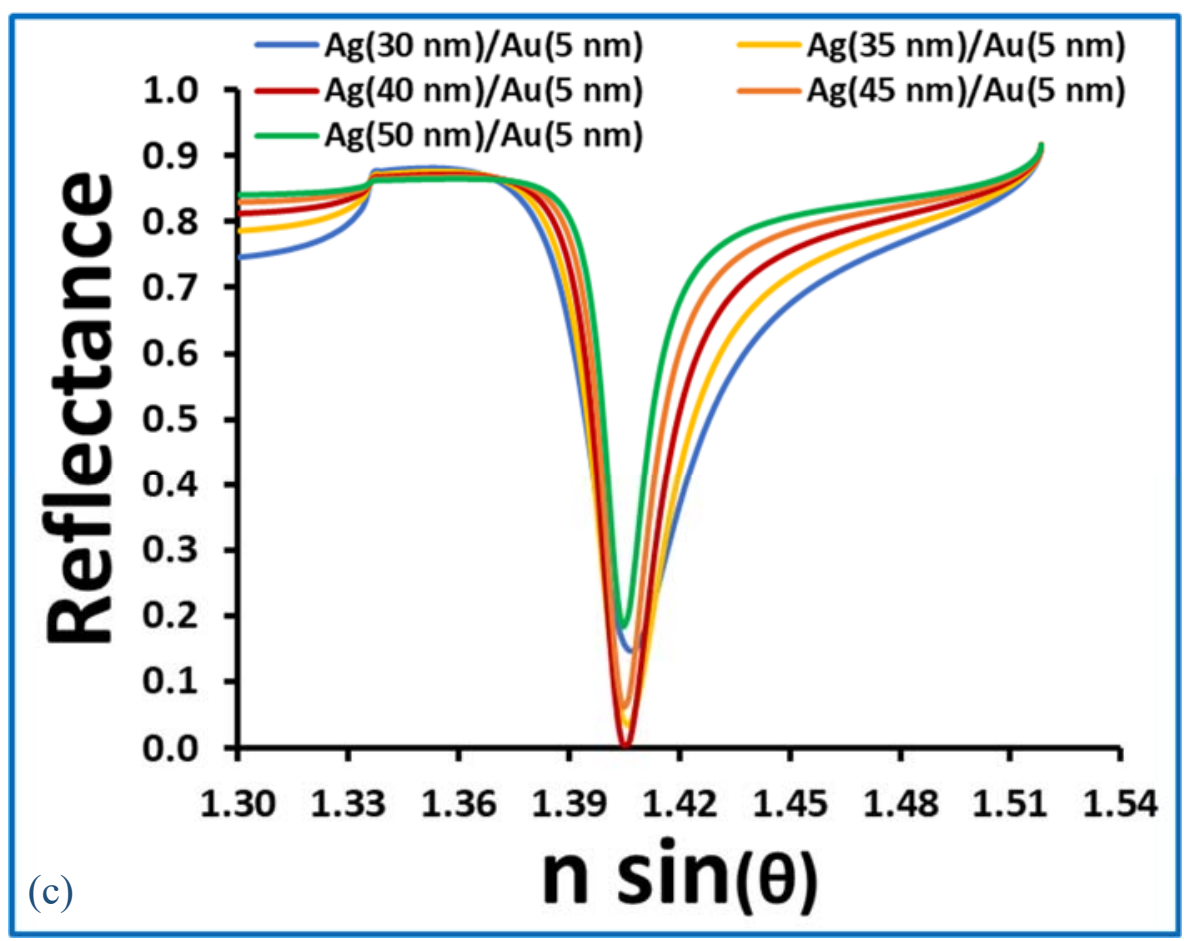

Figure A-4: The calculated SPR reflectance curves for (a) gold thin film, (b) silver thin film with thickness of $30 \mathrm{~nm}, 35 \mathrm{~nm}, 40 \mathrm{~nm}, 45 \mathrm{~nm}$, and $50 \mathrm{~nm}$, and (c) bilayered (silver/gold) thin film with silver thin film thickness of $30 \mathrm{~nm}, 35 \mathrm{~nm}, 40 \mathrm{~nm}, 45 \mathrm{~nm}$, and $50 \mathrm{~nm}$ and a fixed thickness of gold thin film $(5 \mathrm{~nm})$. The following values were used for the calculations: $\mathrm{n}_{\text {prism }}=1.51842, \mathrm{n}_{\text {gold }}=0.38559+2.61439 \mathrm{i}, \mathrm{n}_{\text {silver }}=0.0719+3.2431 \mathrm{i}$, and $\mathrm{n}_{\text {Buffer }}=1.32045$. The excitation wavelength is $660 \mathrm{~nm}$. 

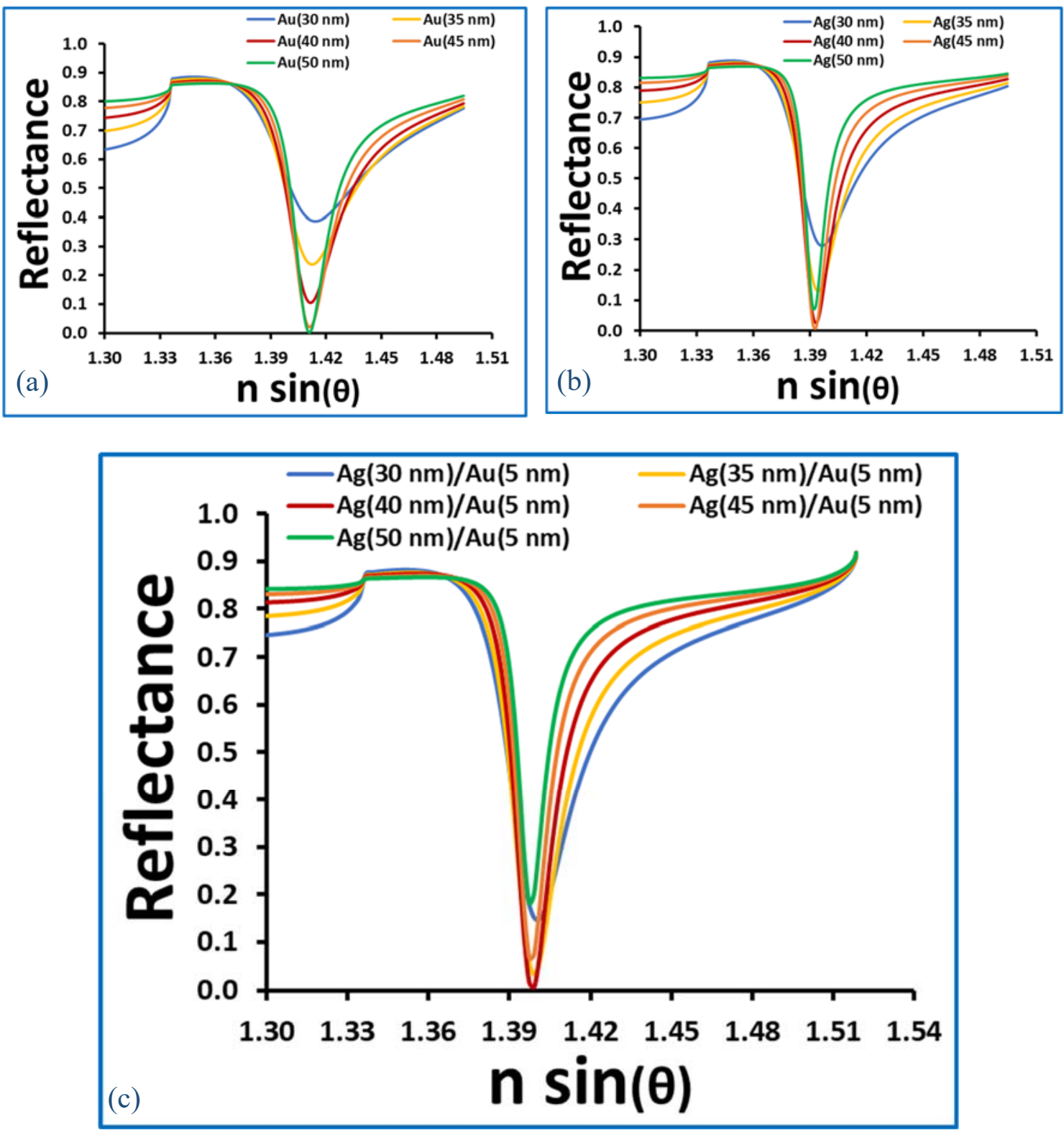

Figure A-5: The calculated SPR reflectance curves for (a) gold thin film, (b) silver thin film with thickness of $30 \mathrm{~nm}, 35 \mathrm{~nm}, 40 \mathrm{~nm}, 45 \mathrm{~nm}$, and $50 \mathrm{~nm}$, and (c) bilayered (silver/gold) thin film with silver thin film thickness of $30 \mathrm{~nm}, 35 \mathrm{~nm}, 40 \mathrm{~nm}, 45 \mathrm{~nm}$, and $50 \mathrm{~nm}$ and a fixed thickness of gold thin film $(5 \mathrm{~nm})$. The following values were used for the calculations: $\mathrm{n}_{\text {prism }}=1.51842, \mathrm{n}_{\text {gold }}=0.38559+2.61439 \mathrm{i}, \mathrm{n}_{\text {silver }}=0.0719+3.2431 \mathrm{i}$, and $\mathrm{n}_{\text {Buffer }}=1.32045$. The excitation wavelength is $685 \mathrm{~nm}$. 

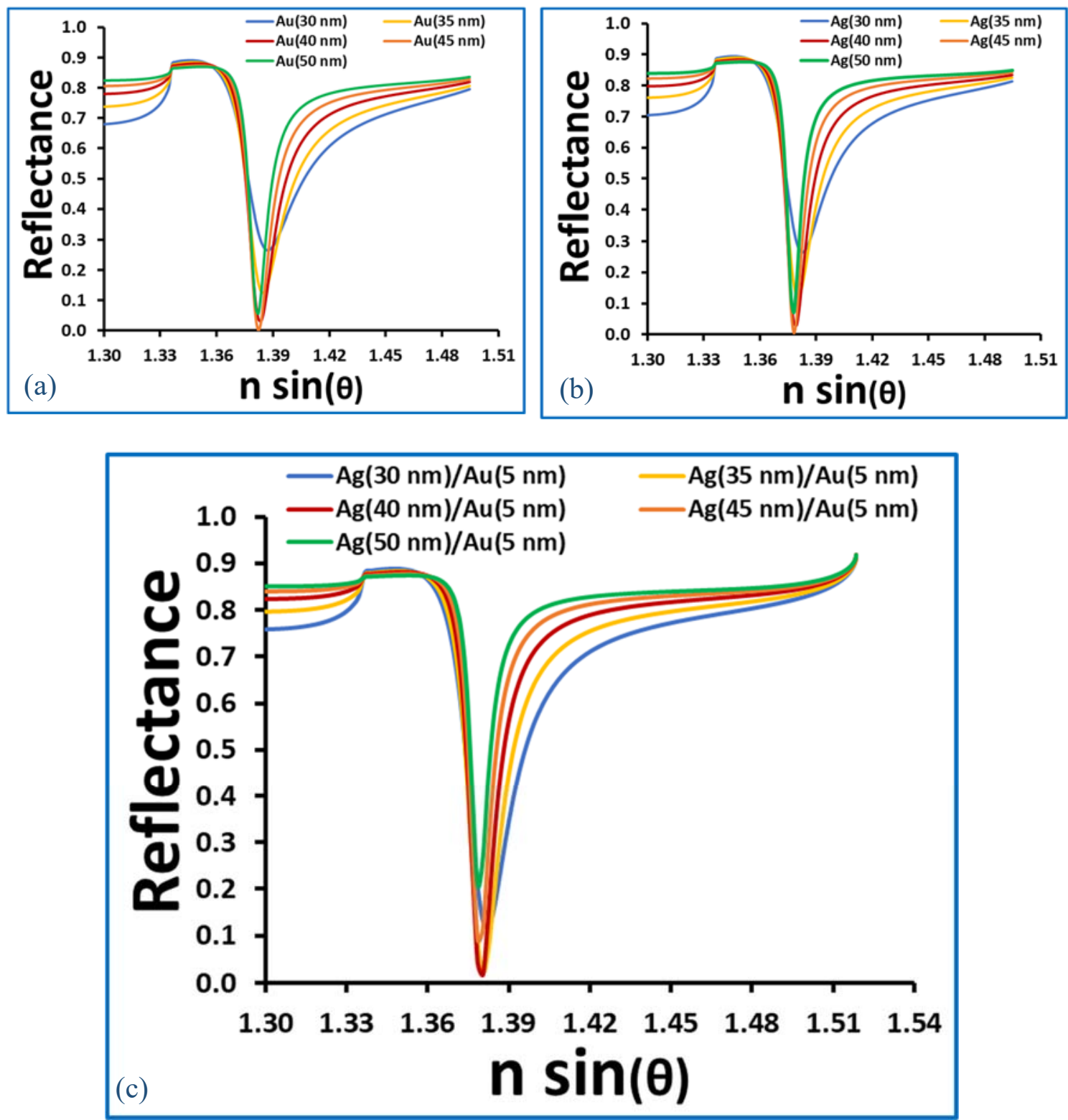

Figure A-6: The calculated SPR reflectance curves for (a) gold thin film, (b) silver thin film with thickness of $30 \mathrm{~nm}, 35 \mathrm{~nm}, 40 \mathrm{~nm}, 45 \mathrm{~nm}$, and $50 \mathrm{~nm}$, and (c) bilayered (silver/gold) thin film with silver thin film thickness of $30 \mathrm{~nm}, 35 \mathrm{~nm}, 40 \mathrm{~nm}, 45 \mathrm{~nm}$, and $50 \mathrm{~nm}$ and a fixed thickness of gold thin film $(5 \mathrm{~nm})$. The following values were used for the calculations: $\mathrm{n}_{\text {prism }}=1.51842$, ngold $=0.38559+2.61439 \mathrm{i}, \mathrm{n}_{\text {silver }}=0.0719+3.2431 \mathrm{i}$, and $\mathrm{n}_{\mathrm{Buffer}}=1.32045$. The excitation wavelength was is $\mathrm{nm}$. 
5. Calculated SPR Reflectance Curves using the Optimal Thickness for Gold, Silver, and Bilayered (silver/gold) Thin Film
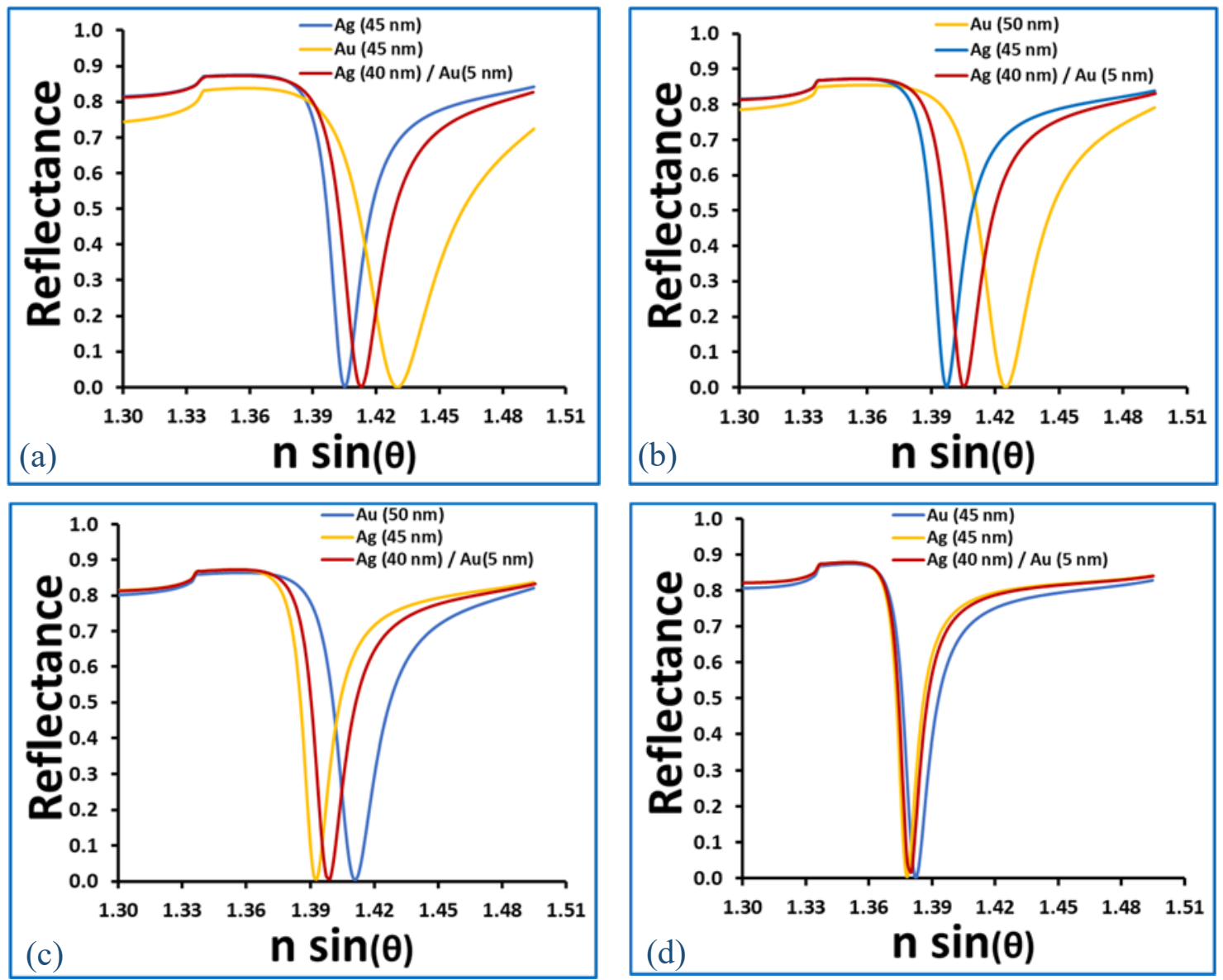

Figure A-7: The calculated SPR reflectance curves using the optimal thickness for the three configurations at (a) $637 \mathrm{~nm}$, (b) $660 \mathrm{~nm}$, (d) $685 \mathrm{~nm}$, and (d) $785 \mathrm{~nm}$. The following values were used for the calculations: $\mathrm{n}_{\text {prism }}=1.51842, \mathrm{n}_{\text {gold }}=0.38559+2.61439 \mathrm{i}, \mathrm{n}_{\text {silver }}=0.0719$ $+3.2431 \mathrm{i}, \mathrm{n}_{\mathrm{Buffer}}=1.32045$.

6. Reflectance under a CV Scan for the Cytochrome c protein Immobilized onto MPABilayered (Ag/Au) Surface 

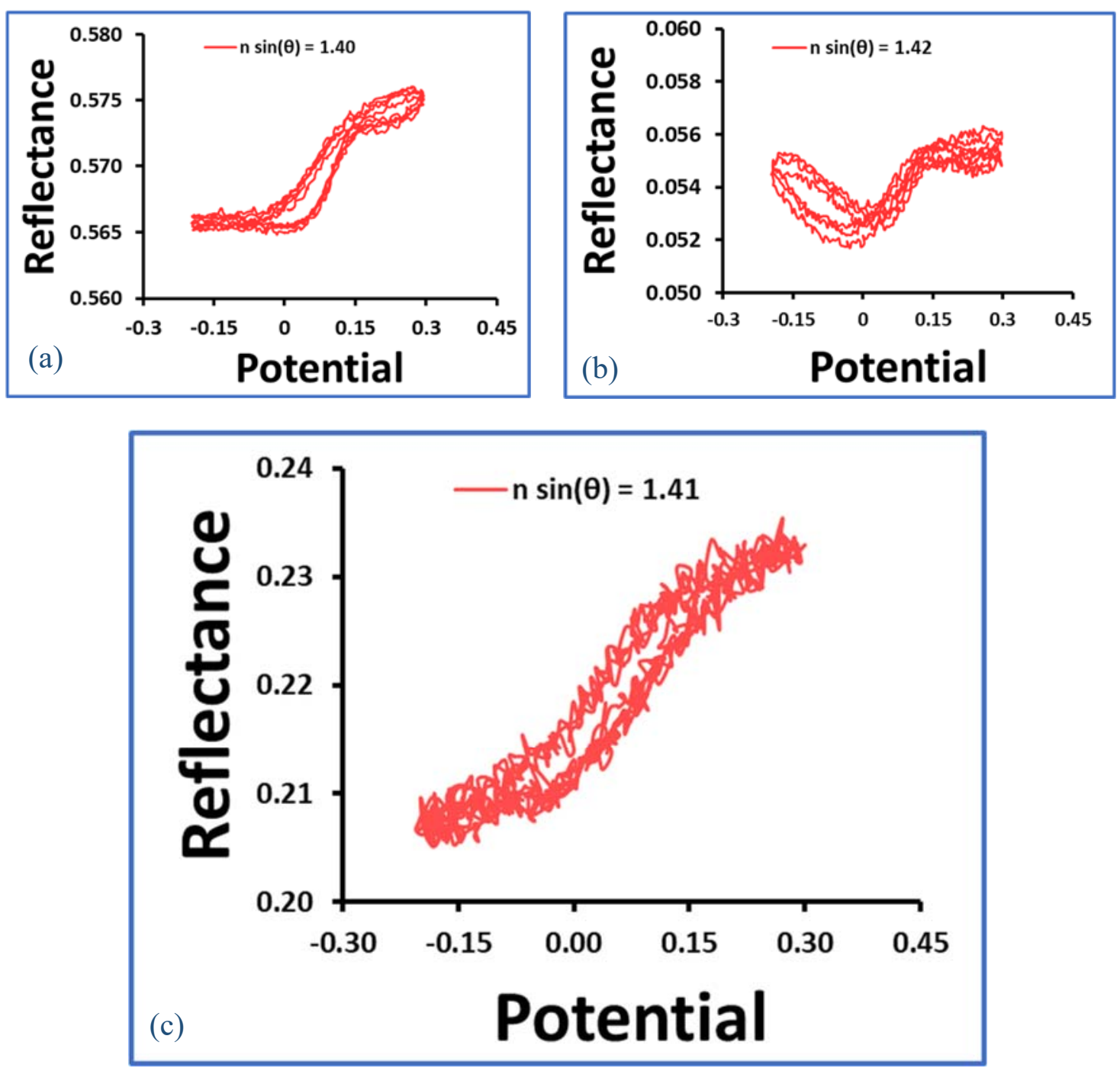

Figure A-8: Reflectance under a CV scan for the cytochrome c protein immobilized onto MPA-bilayered (silver/gold) platform with scan rate of $0.02 \mathrm{~V} / \mathrm{s}$ and at three values of $\mathrm{nsin}(\theta)$ for $637 \mathrm{~nm}$ excitation wavelength.
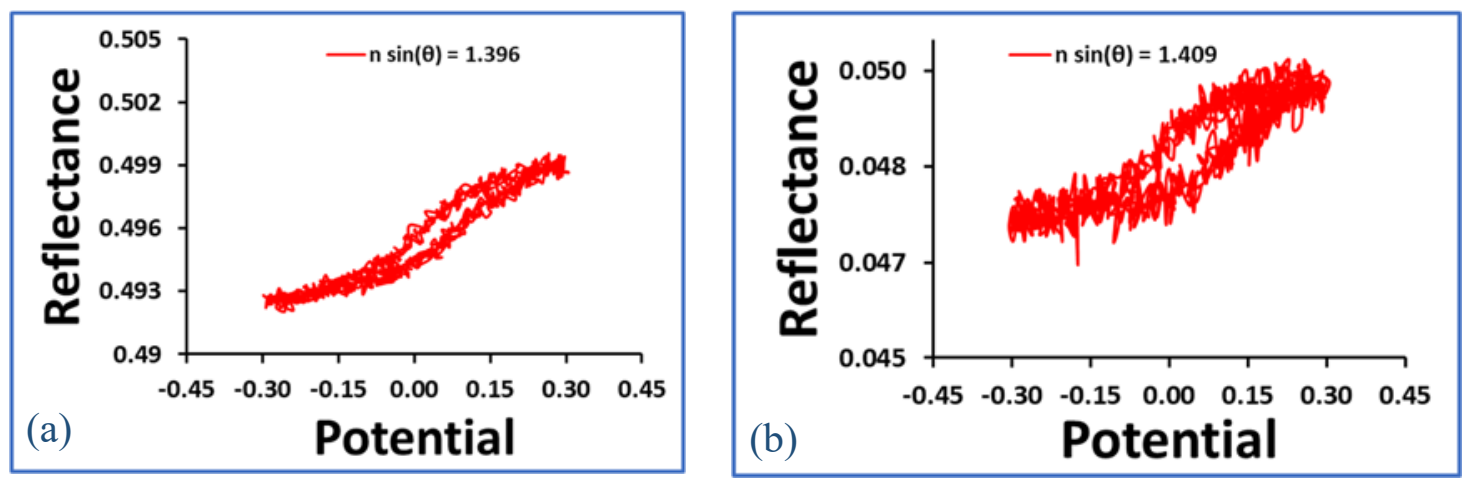


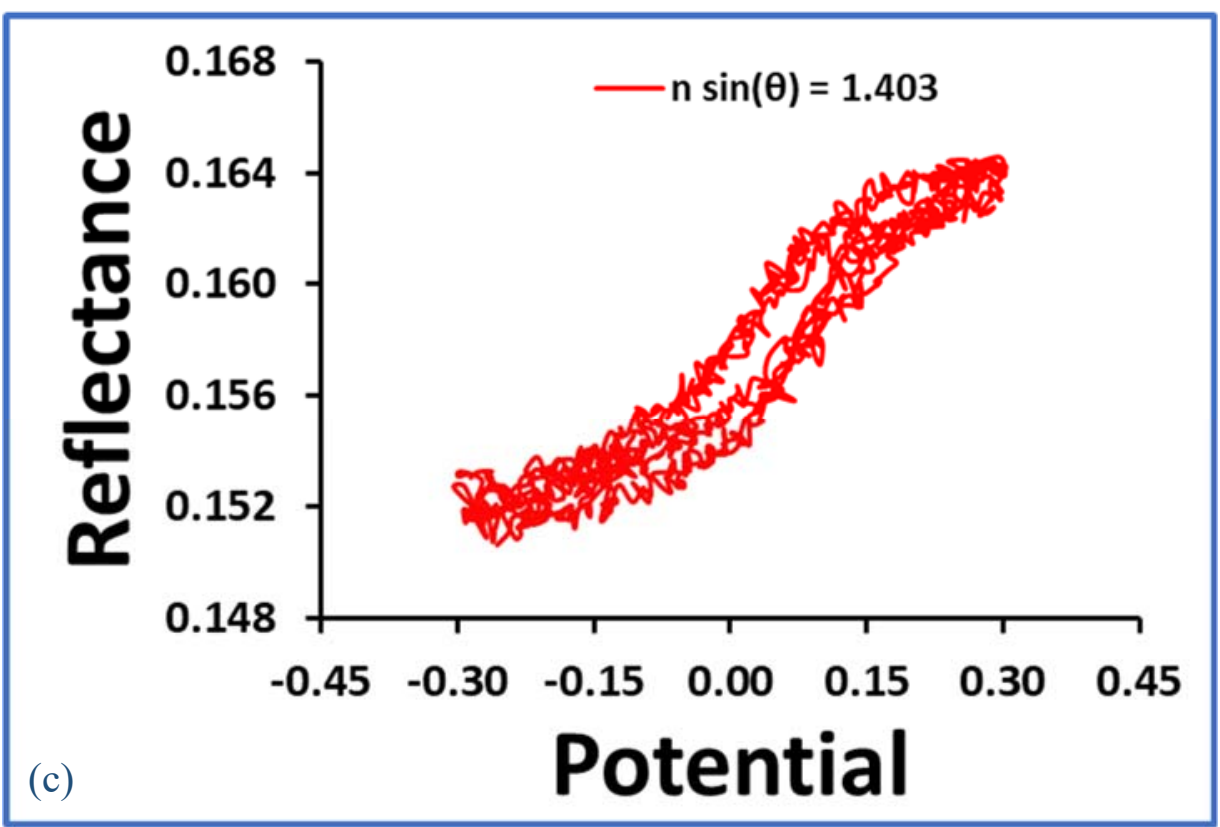

Figure A-9: Reflectance under a CV scan for the cytochrome c protein immobilized onto MPA-bilayered (silver/gold) platform with scan rate of $0.02 \mathrm{~V} / \mathrm{s}$ and at three values of $\mathrm{n} \sin (\theta)$ for $660 \mathrm{~nm}$ excitation wavelength.
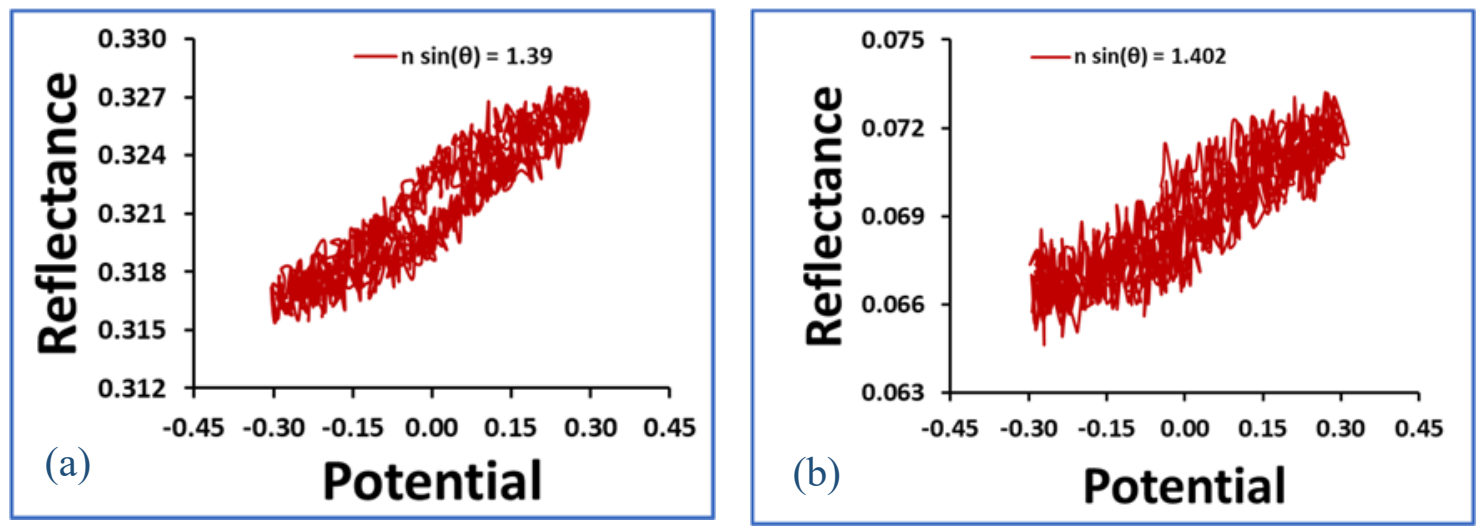


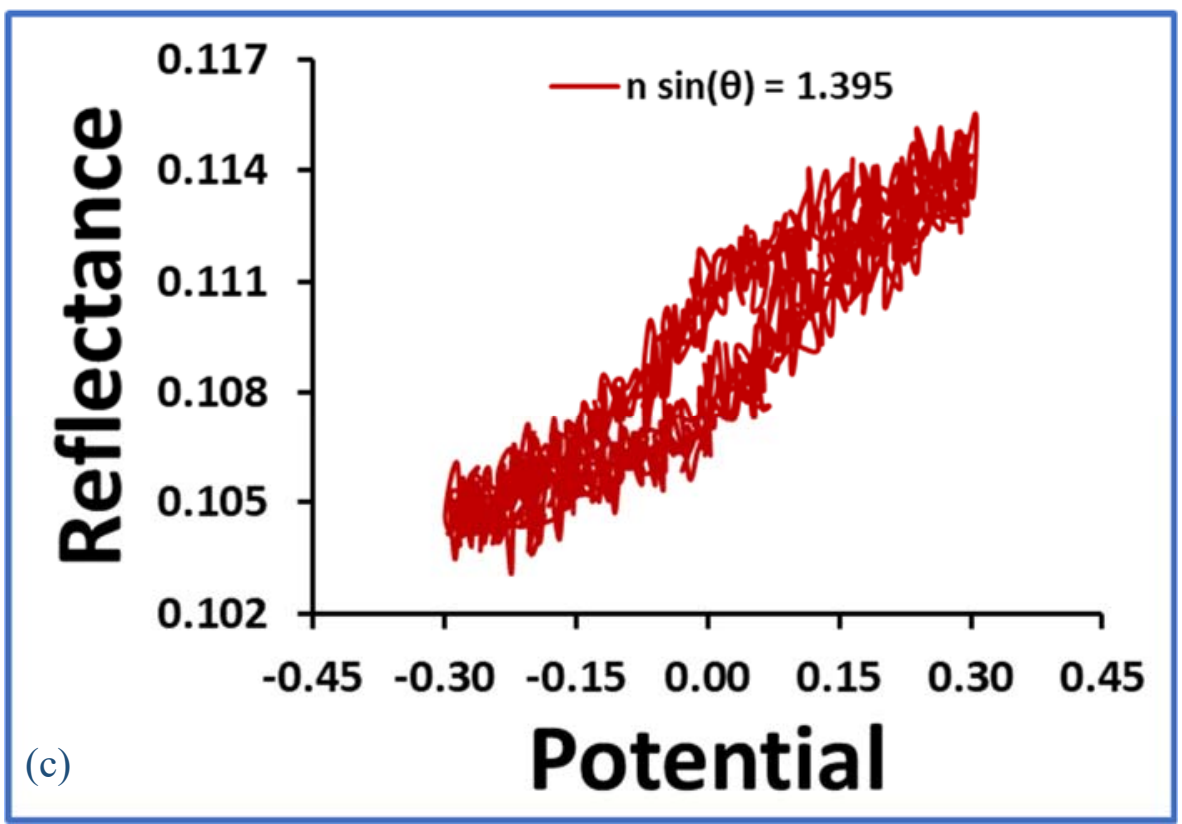

Figure A-10: Reflectance under a CV scan for the cytochrome c protein immobilized onto MPA-bilayered (silver/gold) platform with scan rate of $0.02 \mathrm{~V} / \mathrm{s}$ and at three values of $\mathrm{nsin}(\theta)$ for $685 \mathrm{~nm}$ excitation wavelength.
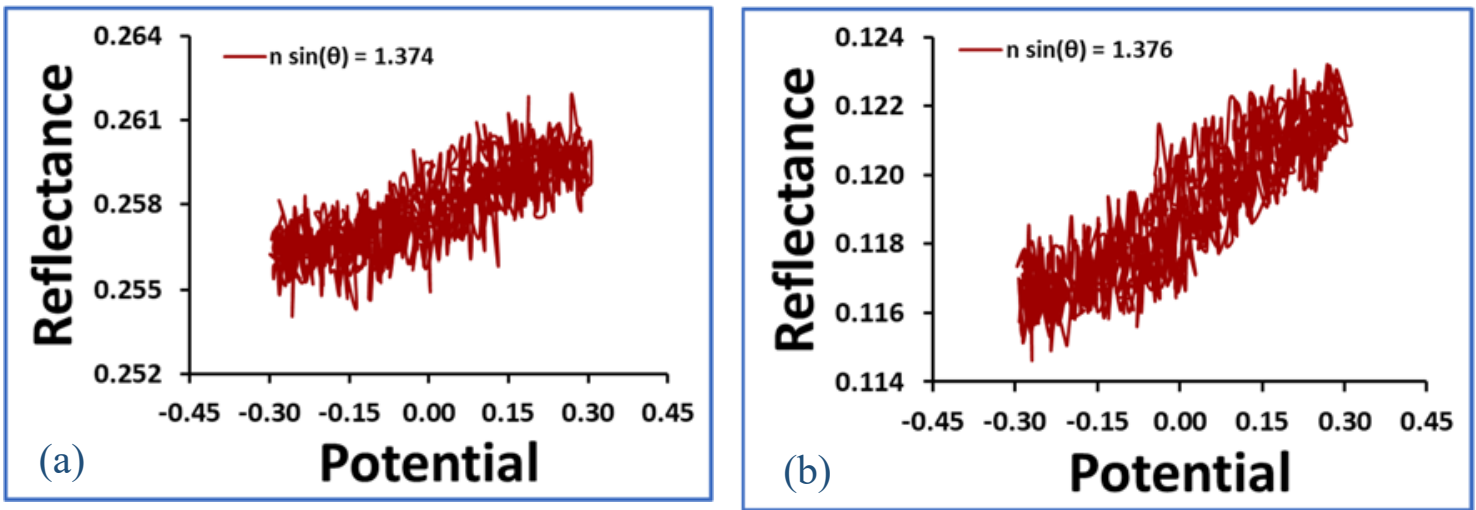


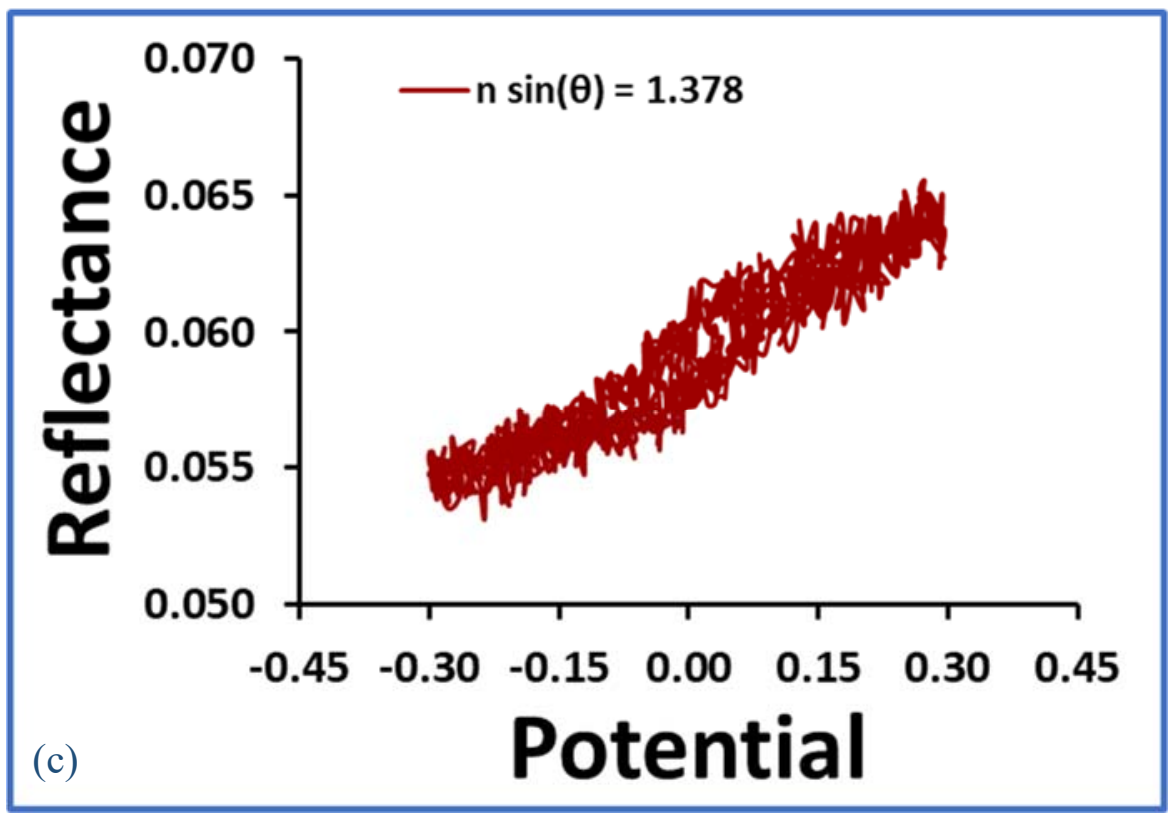

Figure A-11: Reflectance under a CV scan for the cytochrome c protein immobilized onto MPA-bilayered (silver/gold) platform with scan rate of $0.02 \mathrm{~V} / \mathrm{s}$ and at three values of $\mathrm{nsin}(\theta)$ for $785 \mathrm{~nm}$ excitation wavelength.

Appendix B: Electron-Transfer Rate Measurements

1. Cytochrome c protein Directly Immobilized onto the EC-SPR Platform 
Figure B-1 shows the SPR optical signals, $\triangle I_{S P R}$, versus the frequency, $\omega$, of the sinusoidal potential modulation for two samples.

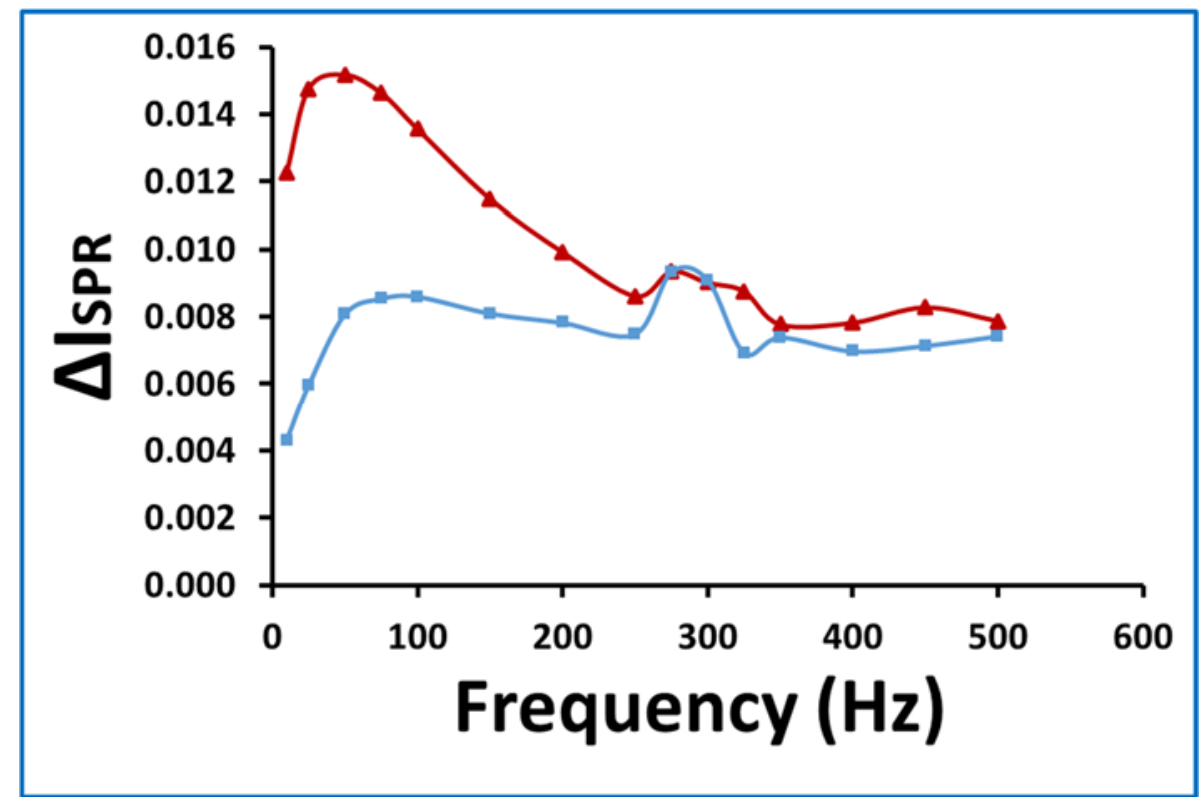

Figure B-1: SPR optical signal, $\triangle \mathrm{I} S P R$, measured under $20 \mathrm{mV}$ AC amplitude of impedance potential modulations at the DC bias potential of $0.094 \mathrm{~V}$ is plotted against angular modulation frequency for two different experiments at the same conditions.

Figure B-2 shows the real and imaginary components of the total electrical impedance of the electrochemical flow cell for cytochrome c protein directly absorbed onto EC-SPR platform measured by a conventional EIS. 

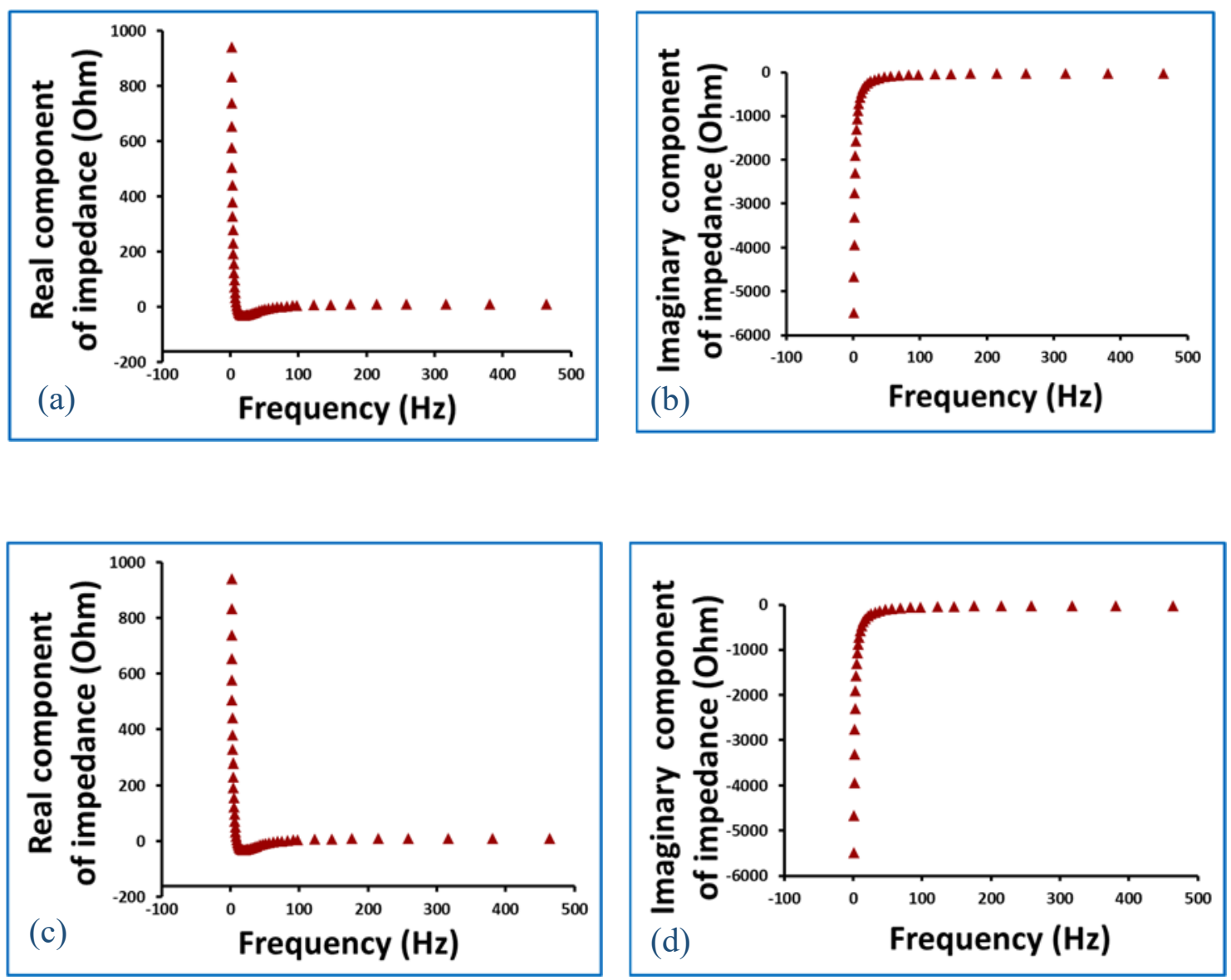

Figure B-2: (a) and (c), represent the real components of the total electrical impedance of the electrochemical flow cell for cytochrome c protein directly absorbed onto EC-SPR platform, while (b) and (d) represent the imaginary components.

Figure B-3 shows a plot of $y=\omega Y^{\prime \prime F}(\omega)$ against $x=2 Y^{\prime F}(\omega)$ calculated over several modulated angular frequencies, $\omega$. The average of the electron-transfer rate for the two experiments were measured to be $(1.7 \pm 0.1) \times 10^{2} s^{-1}$, and $(1.8 \pm 0.2) \times 10^{2} s^{-1}$, respectively. 

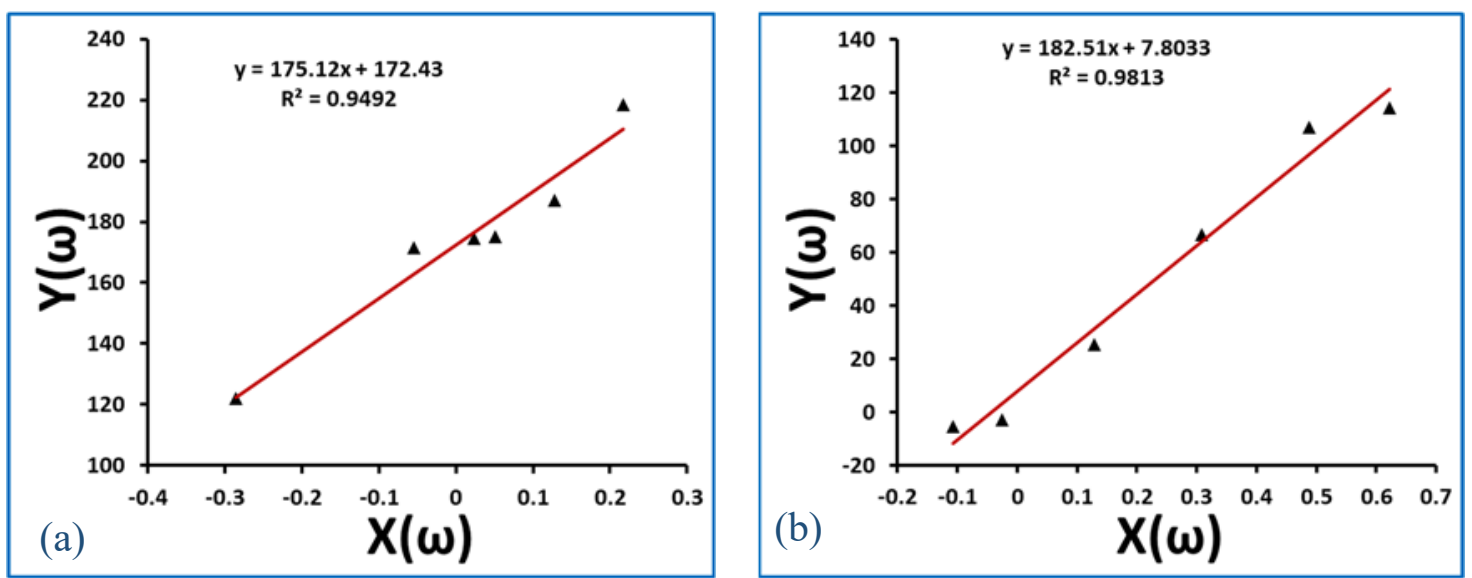

Figure B-3: Electron-transfer rate calculation. The electron-transfer rate is represented by the slope of the linear fit.

\section{Cytochrome c protein Absorbed onto MPA-modified EC-SPR Platform}

Figure 3-5 shows the SPR optical signals, $\triangle I_{S P R}$, versus the frequency, $\omega$, of the sinusoidal potential modulation for two samples. 


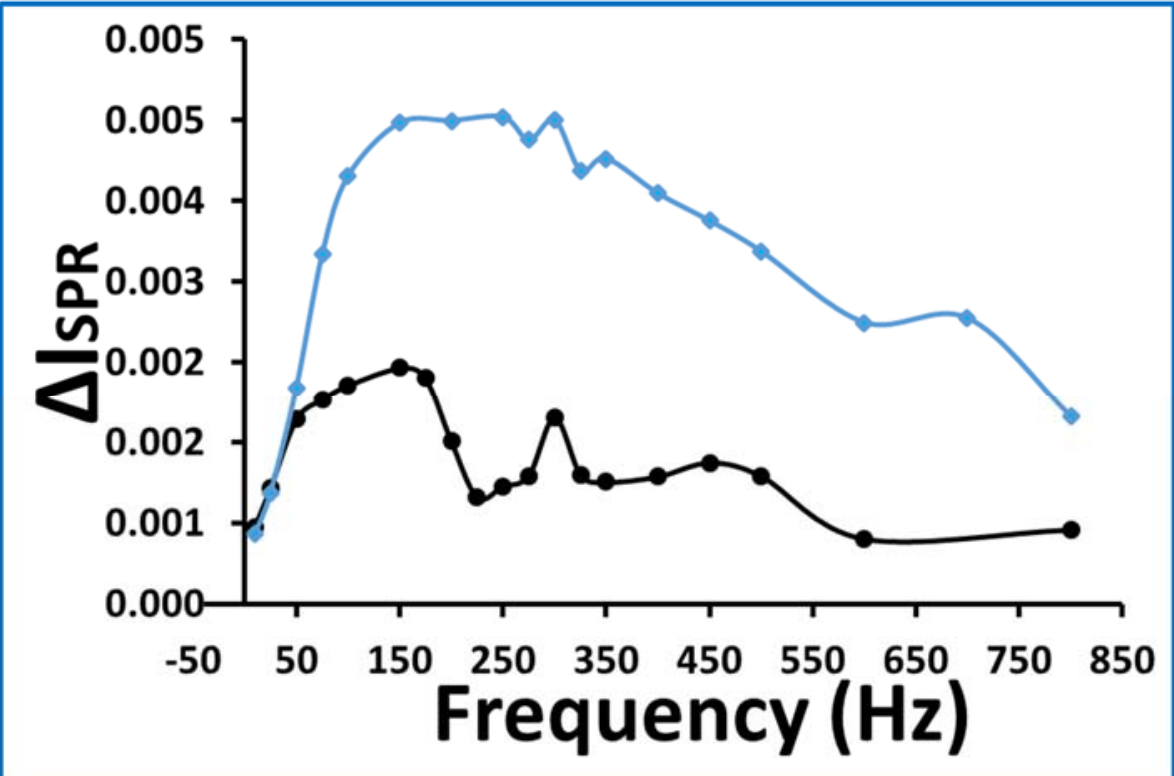

Figure B-4: SPR optical signal, $\triangle I_{S P R}$, measured under $20 \mathrm{mV}$ AC amplitude of impedance potential modulations at the $\mathrm{DC}$ bias potential of $0.011 \mathrm{~V}$ is plotted against angular modulation frequency for two different experiments at the same conditions.

Figure B-5 shows the real and imaginary components of the total electrical impedance of the electrochemical flow cell for electrostatic of absorbed cytochrome $\mathrm{c}$ protein onto the MPA-modified EC-SPR platform measured by a conventional EIS.
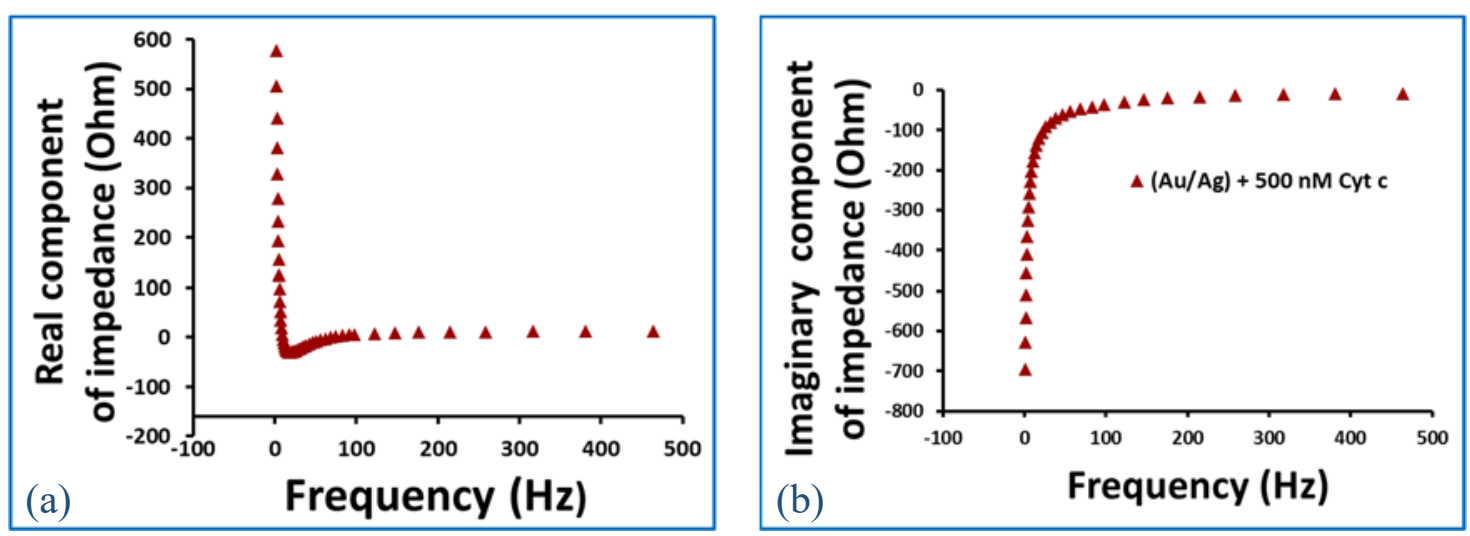

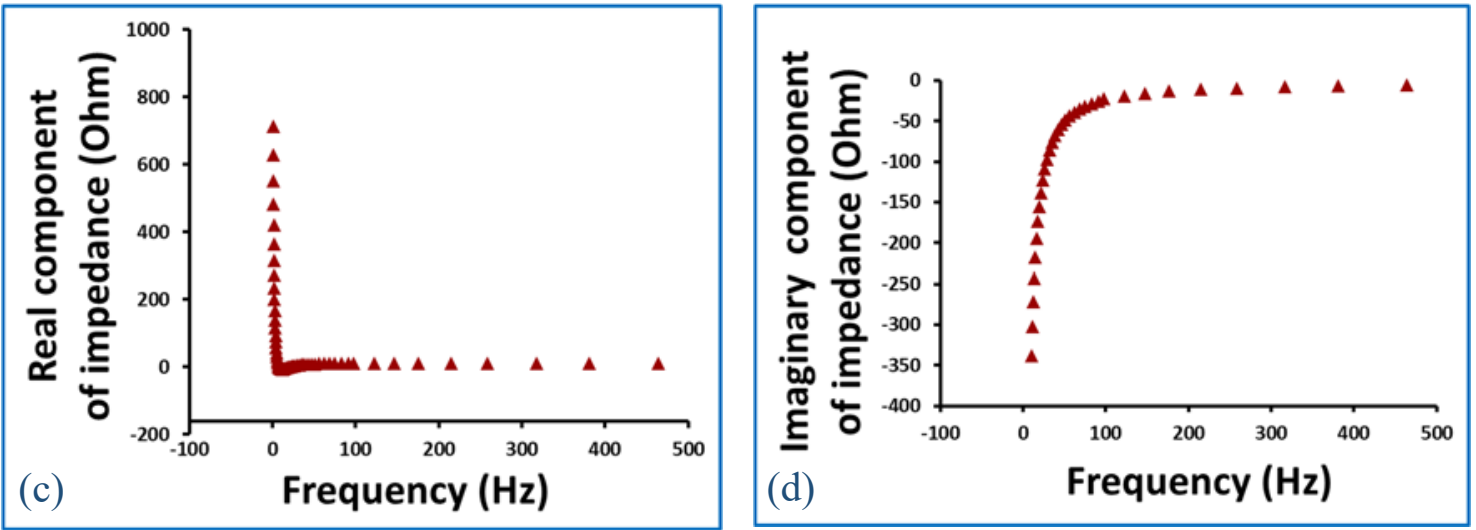

Figure B-5: (a) and (c), represent the real components of the total electrical impedance of the electrochemical flow cell for cytochrome c protein electrostatically absorbed onto MPA modified EC-SPR platform, while (b) and (d) represent the imaginary components.

Figure B-6 shows a plot of $y=\omega Y^{\prime \prime F}(\omega)$ against $x=2 Y^{\prime F}(\omega)$ calculated over several modulated angular frequencies, $\omega$. The average of the electron-transfer rate for the two experiments were measured to be $(4.0 \pm 0.4) \times 10^{2} s^{-1}$, and $(4.2 \pm 0.9) \times 10^{2} s^{-1}$, respectively.
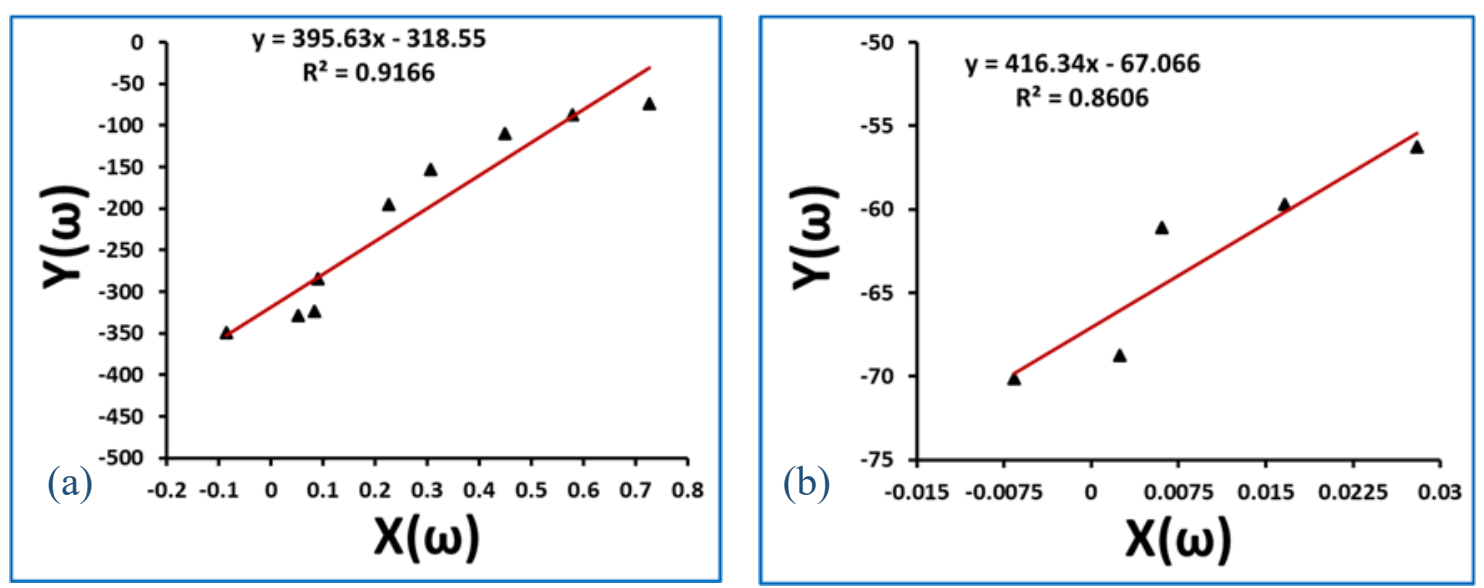

Figure B-6: Electron-transfer rate calculation. The electron-transfer rate is represented by the slope of the linear fit. 


\section{Cytochrome c protein Absorbed onto activated MPA-modified EC-SPR Platform}

Figure B-7 shows the SPR optical signals, $\triangle I S P R$, versus the frequency, $\omega$, of the sinusoidal potential modulation for two samples.

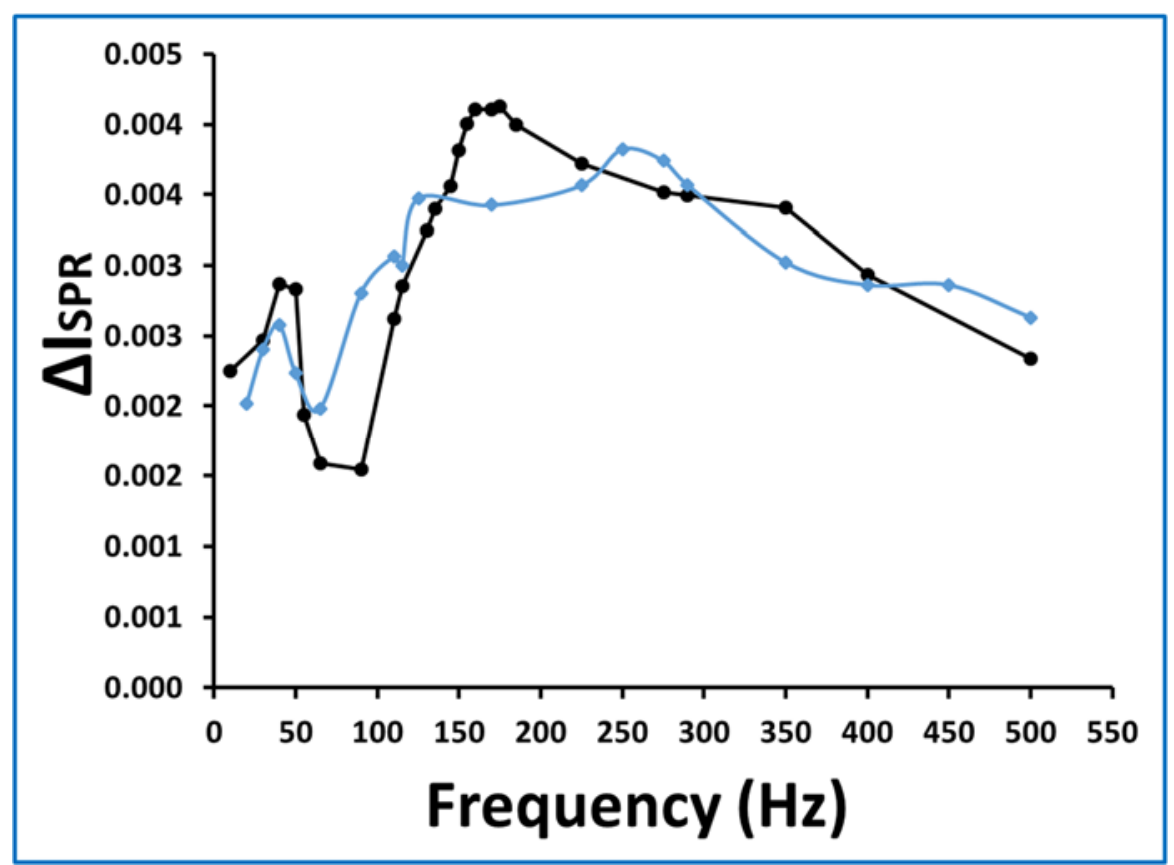

Figure B-7: SPR optical signal, $\triangle I S P R$, measured under $20 \mathrm{mV}$ AC amplitude of impedance potential modulations at the $\mathrm{DC}$ bias potential of $0.0114 \mathrm{~V}$ is plotted against angular modulation frequency for two different experiments at the same conditions.

Figure B-8 shows the real and imaginary components of the total electrical impedance of the electrochemical flow cell for absorbed cytochrome c protein onto the activated MPAmodified EC-SPR platform measured by a conventional EIS. 

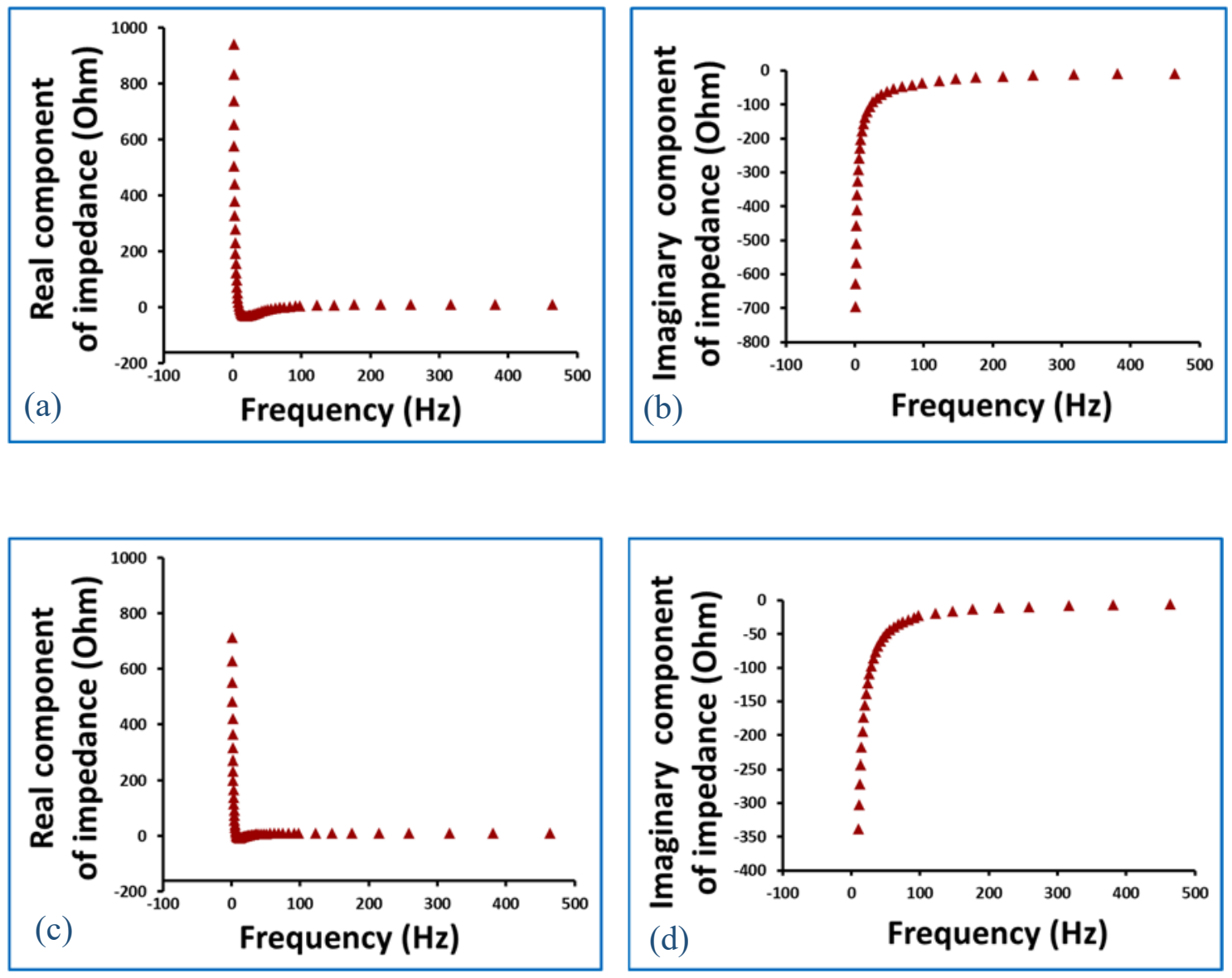

Figure B-8: (a) and (c), represent the real components of the total electrical impedance of the electrochemical flow cell for cytochrome c protein covalently absorbed onto MPA modified ECSPR platform, while (b) and (d) represent the imaginary components.

Figure B-9 shows a plot of $y=\omega Y^{\prime \prime F}(\omega)$ against $x=2 Y^{\prime F}(\omega)$ calculated over several modulated angular frequencies, $\omega$. The average of the electron-transfer rate for the two experiments were measured to be $(4.0 \pm 0.5) \times 10^{2} s^{-1}$, and $(4.3 \pm 0.6) \times 10^{2} s^{-1}$, respectively. 

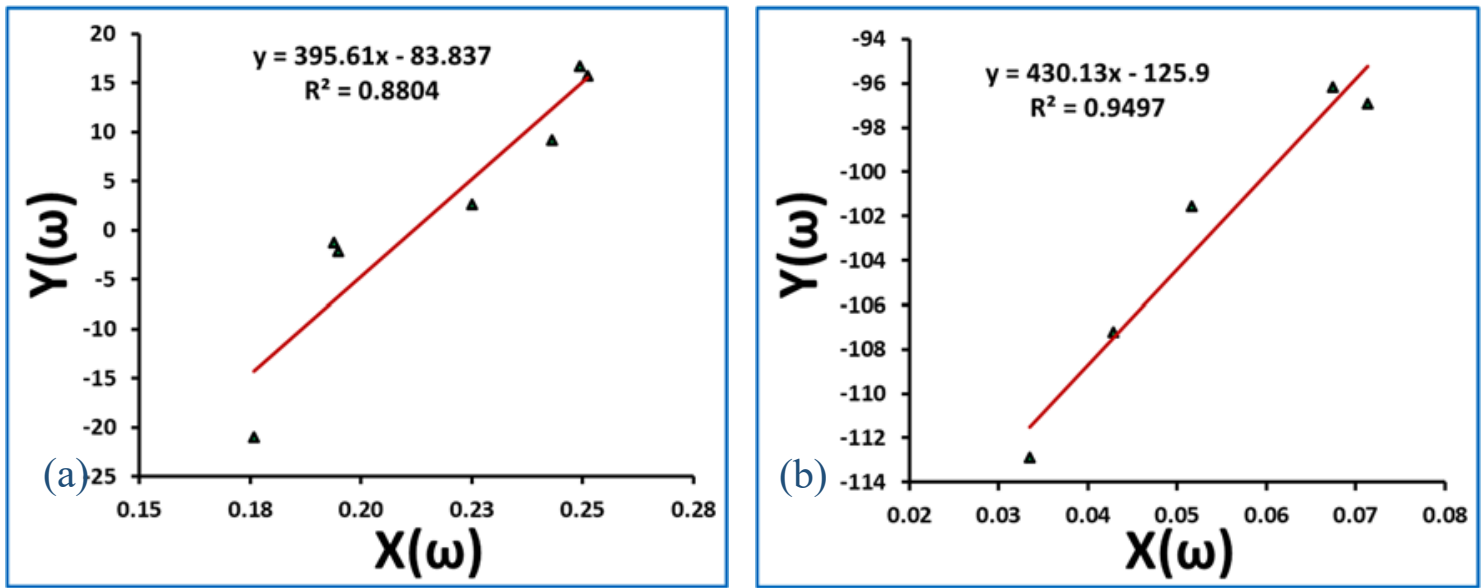

Figure B-9: Electron-transfer rate calculation. The electron-transfer rate is represented by the slope of the linear fit.

4. Cytochrome c protein Absorbed onto Cyt c Ab /CYS-Modified EC-SPR Platform

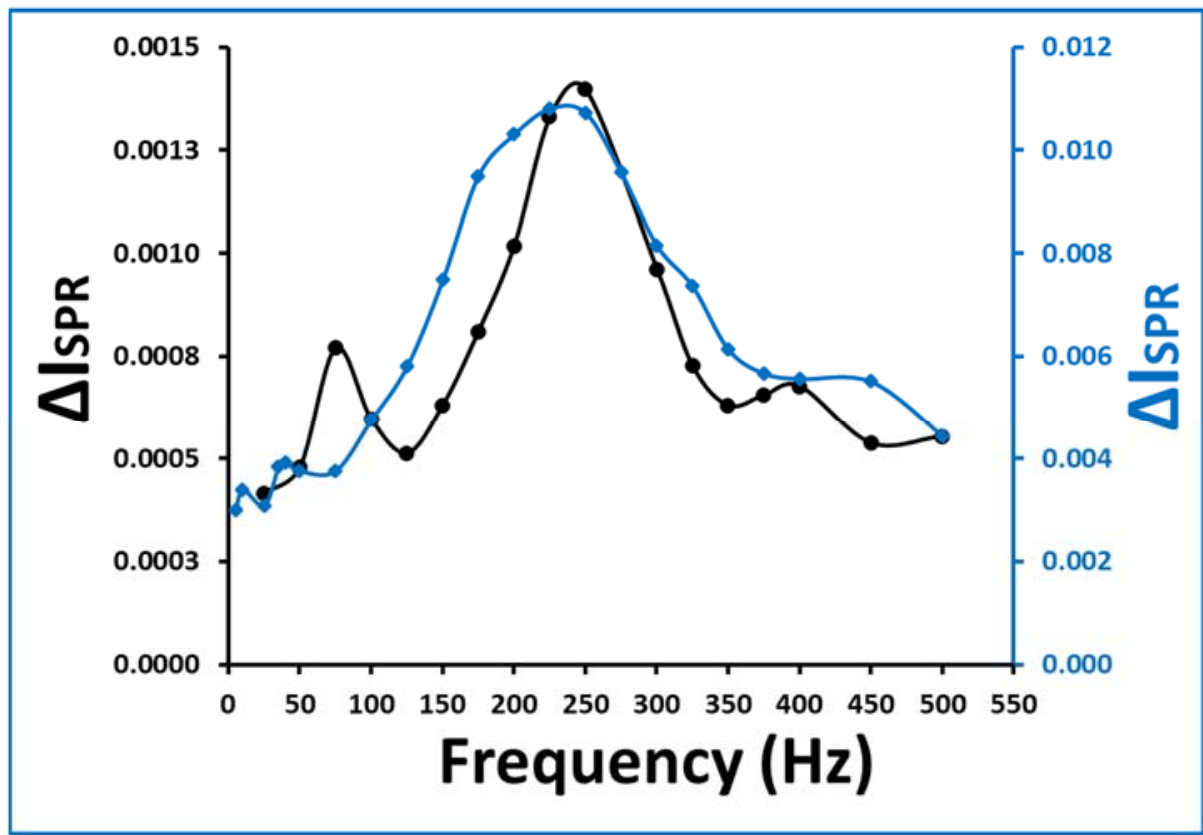

Figure B-10: SPR optical signal, $\triangle \mathrm{I}$ sPR, measured under $20 \mathrm{mV}$ AC amplitude of impedance potential modulations is plotted against angular modulation frequency for two different experiments at the same conditions. 
Figure B-11 shows the real and imaginary components of the impedance for the cytochrome c protein captured by Cyt c Ab.
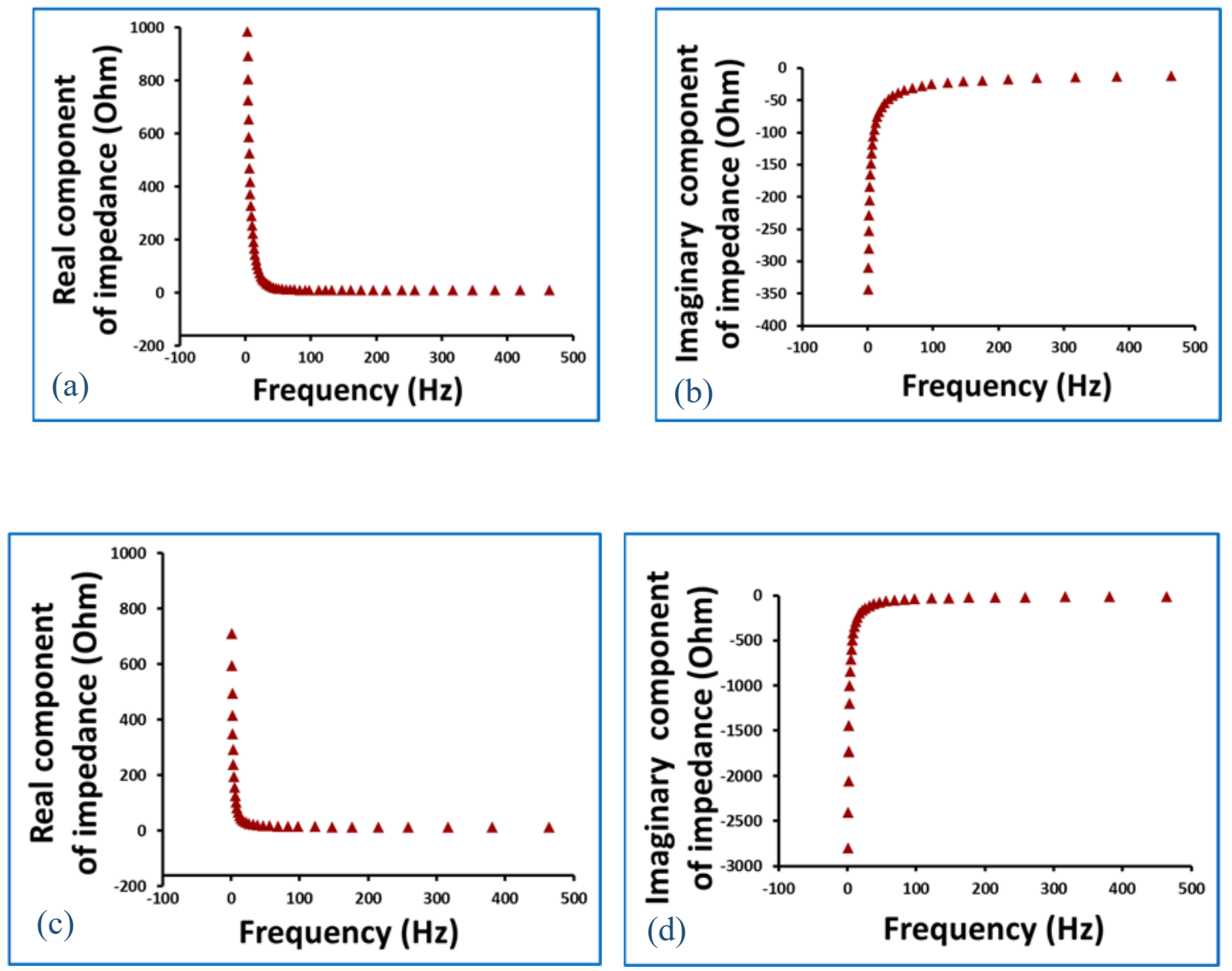

Figure B-11: (a) and (c), represent the real components of the total electrical impedance of the electrochemical flow cell with cytochrome c protein captured by Cyt c Ab, while (b) and (d) represent the imaginary components.

Figure B-12 shows a plot of $y=\omega Y^{\prime \prime F}(\omega)$ against $x=2 Y^{\prime F}(\omega)$ calculated over several modulated angular frequencies, $\omega$. The average of the electron-transfer rate for the two experiments were measured to be $(8.8 \pm 0.5) \times 10^{2} s^{-1}$, and $(7.4 \pm 1.8) \times 10^{2} s^{-1}$, respectively. 

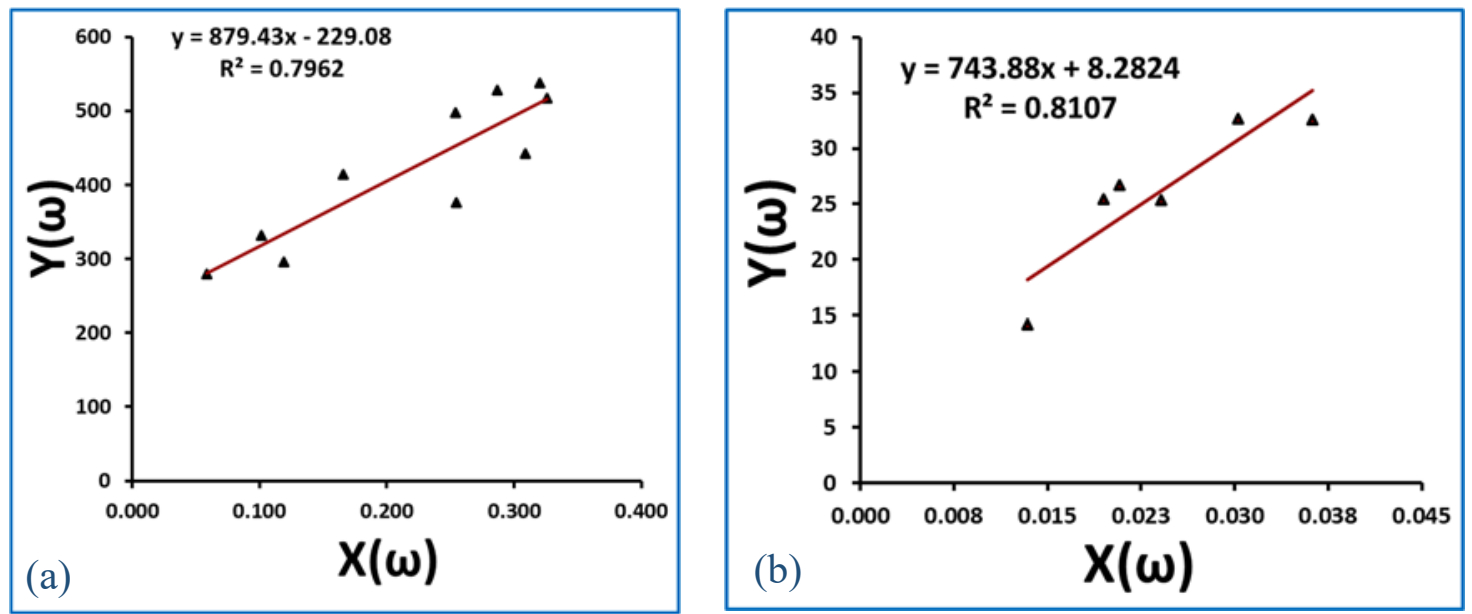

Figure B-12: Electron-transfer rate calculation. The electron-transfer rate is represented by the slope of the linear fit.

5. Cytochrome c protein Absorbed onto Cyt c Ab /CYS-Modified EC-SPR Platform with the presence of the BSA layer

Figure B-13 shows the SPR optical signals, $\triangle I_{S P R}$, versus the frequency, $\omega$, of the sinusoidal potential modulation for two samples. 


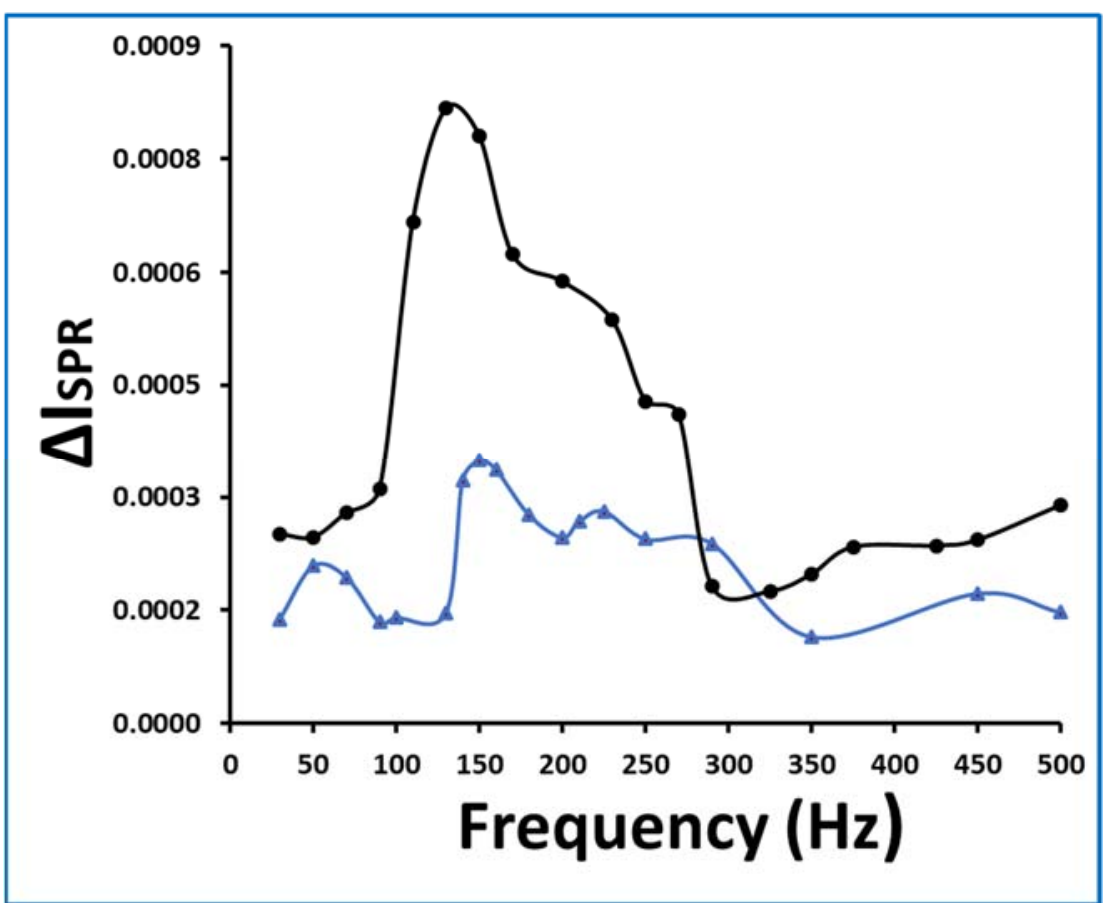

Figure B-13: SPR optical signal, $\triangle \mathrm{I}_{\mathrm{SPR}}$, measured under $20 \mathrm{mV}$ AC amplitude of impedance potential modulations is plotted against angular modulation frequency for two different experiments at the same conditions.

Figure B-14 shows the real and imaginary components of the impedance for the cytochrome $\mathrm{c}$ protein captured by Cyt $\mathrm{c} \mathrm{Ab}$ with the presence of the BSA layer.
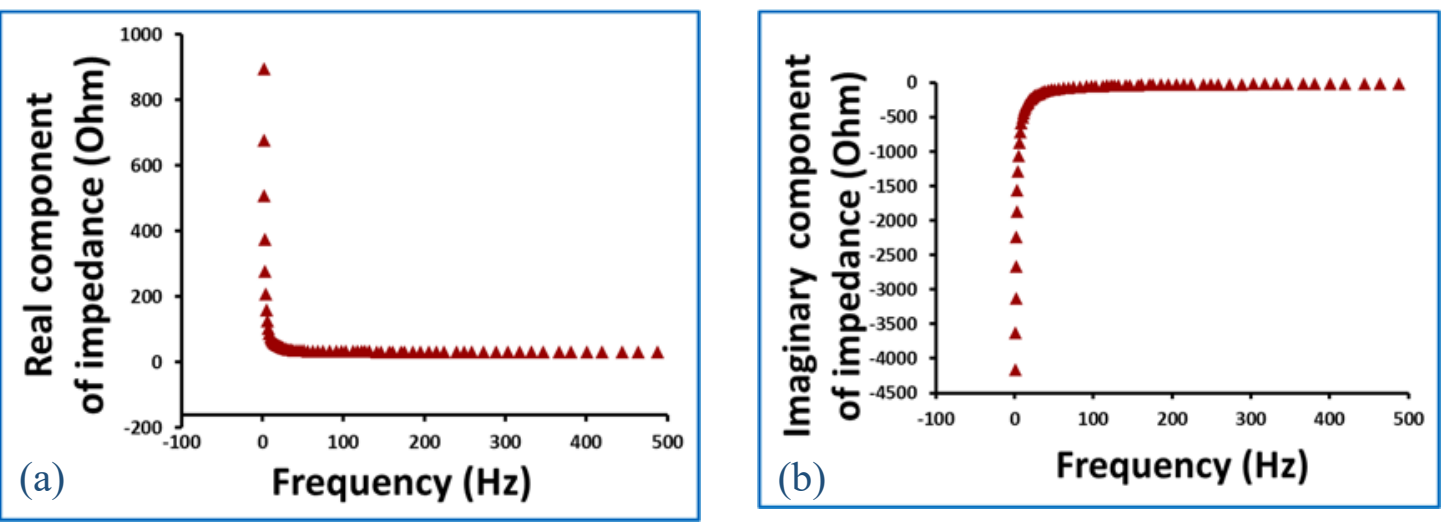

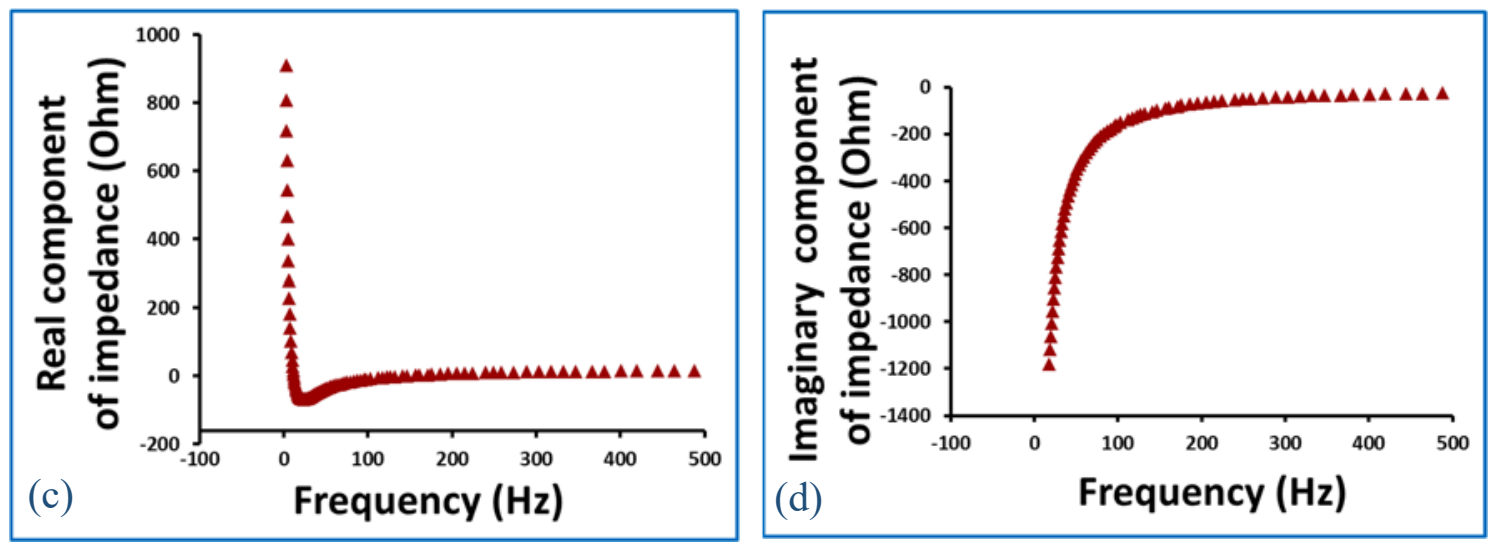

Figure B-14: (a) and (c), represent the real components of the total electrical impedance of the electrochemical flow cell with cytochrome c protein captured by Cyt c Ab with the presence of the BSA layer, while (b) and (d) represent the imaginary components.

Figure B-15 shows a plot of $y=\omega Y^{\prime \prime F}(\omega)$ against $x=2 Y^{\prime F}(\omega)$ calculated over several modulated angular frequencies, $\omega$. The average of the electron-transfer rate for the two experiments were measured to be $(4.1 \pm 0.5) \times 10^{2} s^{-1}$, and $(3.0 \pm 0.9) \times 10^{2} s^{-1}$, respectively.
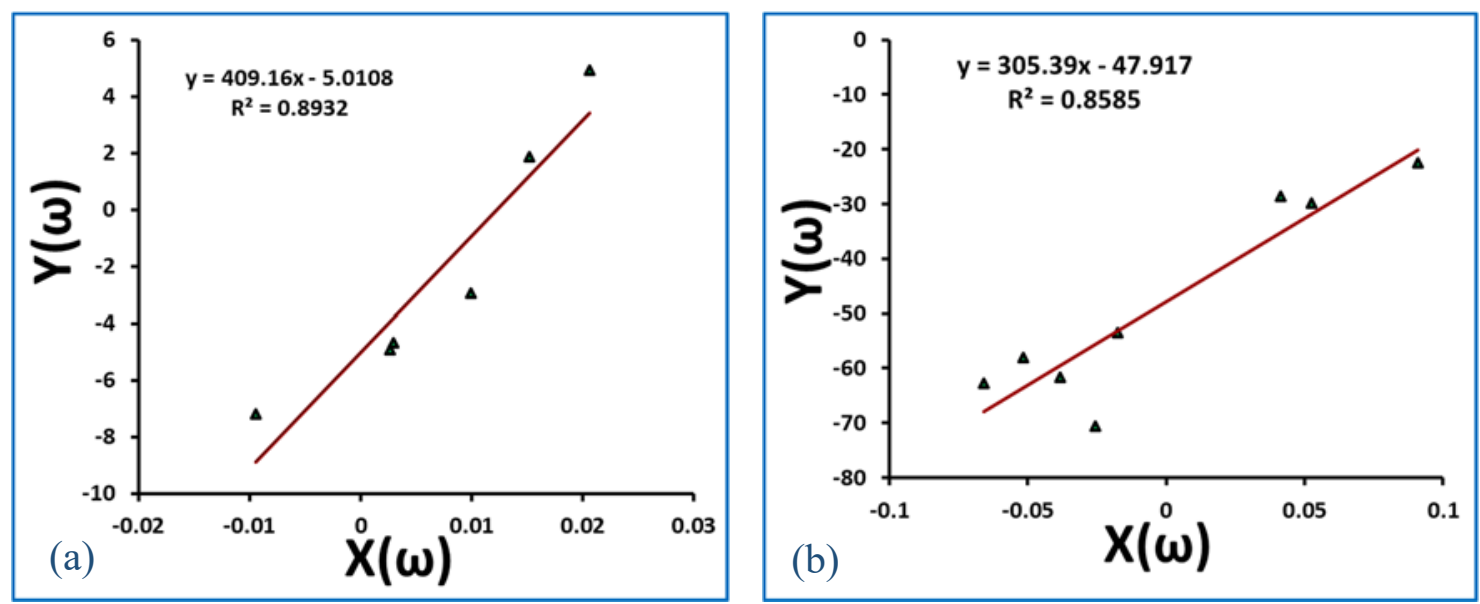

Figure B-15: Electron-transfer rate calculation. The electron-transfer rate is represented by the slope of the linear fit. 


\title{
CURRICULUM VITAE
}

\author{
Aymen Qatamin \\ Department of Physics and Astronomy, \\ University of Louisville, Louisville, KY 40217
}

$(502-631-7120)$

aymen.qatamin@louisville.edu

\section{Qualifications Summary}

- 4+ years hands-on experience in the area of optical film coating and electroactive multilayer fabrication using sputtering coating and ion beam etching processing.

- Hand-on experience in optical, electrical characterization and performance of electroactive integrated surface plasmon resonance (SPR) platforms and electroreflectance (ER) devices.

- 4+years hands-on experience using a variety of optical lab equipment's at the Photonics Labs in the University of Louisville; -mentioning and not restricted to-, lapping and polishing machine, fusion splicers, fiber cleaver, optical spectrum analyzers, power meters, lasers, CCD and ICCD cameras, oscilloscopes, optical stages/mounts, Lock-in amplifier, current amplifier... etc.

- Developed an electrochemical surface plasmon resonance (EC-SPR) strategy for immunosensing detection.

- Developed an electroactive surface plasmon resonance method used to characterize and study electron transfer rates at different layers assemblies.

- Developed a methodology to decrease film absorbance and increase the transparency by a UV-thermal annealing.

\section{TECHNICAL SKILLS AND INSTRUMENTATIONS EXPERTISE}

\section{Computer Software}

- Data Processing: Excel, OriginLab, Python.

- Design: MS office.

\section{Material characterization}


- Electron transfer kinetics of redox active probes using spectroelectrochemical measurements.

- Conduction mechanisms in ultra-thin metal films using 4-probe measurement of resistivity and sheet resistance.

\section{$\underline{\text { Micro-fabrication }}$}

- Thermal evaporator, RF/DC sputtering, ion beam etching.

\section{Equipment ownership Training/Maintenance}

- NOC-4000 dual chamber optical coating system / Nano master.

\section{Education}

Ph.D. Physics

August 2015- Expected April 2020

University of Louisville, Louisville, KY, USA

M.Sc. Physics

2017 University of Louisville, Louisville, KY, USA

M.Sc. Physics

Lund University, Lund, Sweden

\section{B.Sc. Degree Physics}

2001

Al-Mustansiriya University, Baghdad, Iraq

\section{Experience}

\section{University of Louisville}

Research Assistant, Physics and Astronomy Department.

- Developed an electrochemical surface plasmon resonance (EC-SPR) strategy for immunosensing detection.

- Developed an electroactive surface plasmon resonance method used to characterize and study electron transfer rates at different layers assemblies.

- Developed a methodology to decrease film absorbance and transparency by a UV-thermal annealing.

\section{Lund University}

Master student, Department of Physics

2010-2012 
- Developed and studied the performance of the second generation irridum (Ir) coincidence spectrometer in order measure trace quantities of Ir in different geological layers and correlate the age of the layer with different large meteorite impacts.

\section{Tafila Technical University}

Lab Manager, Department of Applied Physics

2006-2010

- Maintains laboratory equipment and arranging equipment replacement, service, and repair.

- Developing and preparing new experiments as well as lecture demonstration equipment.

\section{SELECTED AWARDS}

- Erasmus Mundus Action 2 (Master's program), Lund University, Sweden, 2010.

\section{SELECTED PUBLICATIONS}

- A. H. Qatamin, J. H. Ghithan, M. R. Moreno, B. M. Nunn, F. P. Zamborini, R. S. Keynton, M. G. O'Toole, K. B. Jones and S. B. Mendes, "Detection of influenza virus by electrochemical surface plasmon resonance under potential modulation "Applied Optics, Vol. 58, Issue 11, pp. 2839-2844 (2019).

- J. H. Ghithan, A. H. Qatamin, M. Moreno, B. M. Nunn, R. S. Keynton, M. G. O'Toole and S. B. Mendes, in Immunosensors, The Royal Society of Chemistry, 2019, pp. 42-57.

- A. H. Qatamin, J. H. Ghithan, M. Moreno, B. M. Nunn, K. B. Jones, F. P. Zamborini, R. S. Keynton, M. G. O'toole, and S. B. Mendes, "Detection of influenza virus by electrochemical surface plasmon resonance under potential modulation," in Optical Sensors and Sensing Congress (ES, FTS, HISE, Sensors), OSA Technical Digest (Optical Society of America, 2019), paper SW4C.4 